\title{
WestVirginiaUniversity
}

THE RESEARCH REPOSITORY @ WVU

Graduate Theses, Dissertations, and Problem Reports

2009

\section{Nonlinear dynamics modulation in a neon glow discharge plasma}

Paul M. Miller

West Virginia University

Follow this and additional works at: https://researchrepository.wvu.edu/etd

\section{Recommended Citation}

Miller, Paul M., "Nonlinear dynamics modulation in a neon glow discharge plasma" (2009). Graduate Theses, Dissertations, and Problem Reports. 2854.

https://researchrepository.wvu.edu/etd/2854

This Dissertation is protected by copyright and/or related rights. It has been brought to you by the The Research Repository @ WVU with permission from the rights-holder(s). You are free to use this Dissertation in any way that is permitted by the copyright and related rights legislation that applies to your use. For other uses you must obtain permission from the rights-holder(s) directly, unless additional rights are indicated by a Creative Commons license in the record and/ or on the work itself. This Dissertation has been accepted for inclusion in WVU Graduate Theses, Dissertations, and Problem Reports collection by an authorized administrator of The Research Repository @ WVU.

For more information, please contact researchrepository@mail.wvu.edu. 


\title{
Nonlinear Dynamics Modulation in a NeOn Glow Discharge Plasma
}

\author{
Paul M. Miller \\ Dissertation submitted to the Eberly College of Arts and Sciences \\ at West Virginia University \\ In partial fulfillment of the requirements for the degree of
}

Doctor of Philosophy

in

Physics

\author{
Mark E. Koepke, Ph.D., Chair \\ Duncan R. Lorimer, Ph.D. \\ Earl E. Scime, Ph.D. \\ Charter D. Stinespring, Ph.D. \\ Dimitris Vassiliadis, Ph.D. \\ H. Arthur Weldon, Ph.D.
}

Department of Physics
Morgantown, West Virginia 2009

Keywords: dynamics modulation, nonlinear dynamics, wave competition, van der Pol equation, periodic pulling, mode transition

C)2009 Paul M. Miller 


\title{
Abstract \\ Nonlinear Dynamics Modulation in a Neon Glow Discharge Plasma
}

\author{
Paul M. Miller
}

In dynamics modulation, two modes in a driven neon glow discharge alternate as the dominant mode as their response to the driving force alternates between spatiotemporal and temporal periodic pulling. This phenomenon was first noted by Koepke, Weltmann, and Selcher (Bull. Am. Phys. Soc. 40, 1716 (1995)), who saw two limited but representative cases and proposed a mechanism (Phys. Rev. E 62, 2773 (2000)) by which it occurs. The intent of this dissertation is to document experimentally and test the dynamics modulation mechanism they proposed. Using a new extension of a previous mathematical treatment of periodic pulling, the resulting experimental data are used to verify the predicted mechanism. A numerical model is also presented that reproduces the signature of dynamics modulation and further supports the validity of the mechanism.

For two pairs of mode frequencies, three complete data series as driving frequency is increased are presented. Each of these data series shows the progression of the system from pure spatiotemporal behavior, through dynamics modulation, and ending at entrainment in the upper mode. Ionization wave modes are examined using time series recorded using a photodiode with a narrow band filter that selectively passes the primary neon spectral line at $640 \mathrm{~nm}$. The system was periodically driven using a narrow-band ring dye laser tuned to a wavelength near the metastable neon transition at $588.35 \mathrm{~nm}$. The amplitude of the driving force was decreased (increased) by tuning the laser away from (nearer to) the center of the neon line, while the driving frequency was controlled by an acousto-optic modulator chopping the laser beam at the desired frequency. Arnol'd tongue boundaries identifying the edges of frequency entrainment regions in the driving amplitude-driving frequency plane were established for four different discharge currents. The (upward) dynamics modulation behavior seen by Koepke, Weltmann, and Selcher was reproduced and additional data were acquired for two additional representative cases of downward modulations, previously undocumented. The upward modulations are used to verify the mechanism, while the downward modulations exhibit qualitatively different behavior. These differences are discussed.

Two coupled van der Pol equations were chosen to model the mechanism described by Koepke, Weltmann, and Selcher, and the resulting time series was solved with a Runge-Kutta routine whose parameters could be adjusted as the simulation proceeded. The model successfully reproduces the qualitative behavior of dynamics modulation and reinforces the experimental verification of the proposed mechanism, but lacks sufficient complexity for a complete quantitative comparison. 


\section{Acknowledgements}

In the summer of 2002, I found myself scraping paint on a ladder outside the southeast side of my home and decided to look into completing my PhD studies. Later that summer, my wife and I were invited to a pig roast hosted by Dr. Mark Koepke and Denise Knoebel, and I mentioned that I was interested in doing some research. That was all that it took. My idea became a paid summer research job the following summer, with support from Dr. Koepke's acquisition of a Research Experience for Teachers grant. This was my first exposure to the quickness with which Dr. Koepke generates ideas, to his enthusiasm for projects, and to his tendency to go out of his way to accommodate others' needs.

When the sequence of plasma classes started again during spring semester 2004, Dr. Koepke adjusted the schedule to allow me to attend after my regular teaching day at University High School and arranged for a tuition waiver to make my decision to continue even easier. This continued throughout the four-course series. I am grateful to Dr. Koepke (and to Dr. Earl Scime and my fellow students) for changing the course schedule solely for my benefit.

As I learned plasma physics, I worked to relearn the basic physics I had learned in the early 1990s so that I could take the three qualifiers. With these passed and my coursework essentially complete, I decided to leave teaching at the end of the 2005-2006 academic year and pursue my Ph.D. full time. This dissertation is the culmination of this effort. I must acknowledge the guidance, assistance, and patience of many people at the end of this process, but the first thanks go to Dr. Koepke. He recognized and nourished my interest, found a way to accommodate the special circumstances that I brought as a non-traditional student and a public school science teacher, and ultimately directed me to this ideal project. He discovered and named dynamics modulation, but set aside the complete investigation for one of his students. I am pleased and privileged to be the one to take it on. All aspects of this work reflect my debt to him.

Thanks to Dr. Earl Scime for his guidance, advice, and interest in my progress. Special thanks are owed to Dr. Scime for serving as chair for my defense and for carefully and efficiently acquiring the necessary approval for this emergency change.

Thanks to Dr. Duncan Lorimer, Dr. Charter Stinespring, and Dr. Art Weldon for reviewing this work and serving on my graduate committee. Special thanks to Dr. Dimitris Vassiliadis for his 
kind instruction and advice during the final stages of the project and for serving as a committee member with little notice.

I also extend thanks to Dr. Herbert Gunell, whose advice and mentorship have improved all I have done over the past two years. I appreciate his thoughtful and practical approach to scientific problems, his genuine interest in plasma physics, and his keen sense of humor. I will miss our Thursday lunches, and I wish you the best as we both leave the WVU Plasma Physics lab.

A debt of gratitude is owed to Dr. K. -D. Weltmann with whom Dr. Koepke arranged the relocation of the glow discharge tube used in this thesis from the University of Greifswald to West Virginia University.

Thanks to Dr. John Zielinski for setting up and showing me the nonlinear UJT circuit from Koepke and Hartley (1991).

Thanks to Eric Reynolds for teaching me how to operate, troubleshoot, and repair the WVU $Q$-machine. Thanks to Sean Finnegan for help with occasional theory or computation questions and for the $\mathrm{ATT}_{\mathrm{E}} \mathrm{X}$ dissertation template. Thanks to Mike Zimmerman and Josh Miller for learning experimental techniques alongside me. Thanks also to Chris Compton, Rob Hardin, Jon Blessington, Saikat Chakraborty Thakur, Jeff Walker, Mattias Törnquist, Olga Volotskova, and Stephanie Sears for sharing parts of the journey. Thanks to all my fellow students for their friendship.

My sincere thanks to Carl Weber, Doug Mathess, Tom Milam, and Phil Tucker for their advice, help and patience in the shop, and to Sherry Puskar, Siobhan Byrne, and Sandy Johns for their capable and friendly help in the office.

I want to express my love and thanks to Richard and Retha Miller for being excellent parents and supporting my reading habits and varied interests while growing up. The better parts of my talent and character come from them.

This work is dedicated to my family. I am so proud of my funny and talented son Griffin. Thanks to him for giving up playtime to sit in the lab with me on snow days and evenings and for being such a terrific kid. Thanks also to new little Nora Wren, who smiles generously and provides the purest inspiration. This whole adventure would not have occurred without the unwavering support of my wife Betsy. She always encouraged me, and she sacrificed much so that I could complete this adventure. I am fortunate to have such a wonderful family and look forward to many more years with them. 


\section{Table of Contents}

$\begin{array}{ll}\text { Abstract } & \text { ii }\end{array}$

Acknowledgements $\quad$ iii

List of Tables

$\begin{array}{ll}\text { List of Figures } & \text { ix }\end{array}$

I Introduction $\quad 1$

A. Glow discharge physics . . . . . . . . . . . . . . . . . 1

1. A brief history of the plasma glow discharge . . . . . . . . . . . . 1

2. Basic features of an undriven discharge tube . . . . . . . . . . . 2

3. Ionization waves . . . . . . . . . . . . . . . 3

B. Nonlinear dynamics . . . . . . . . . . . . . . . . . . . 6

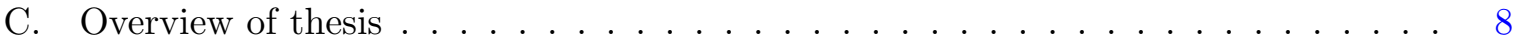

1. Presented work . . . . . . . . . . . . . . . . . . . 8

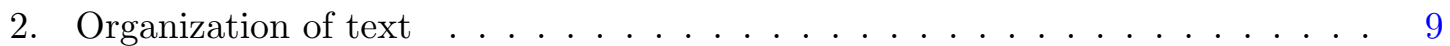

$\begin{array}{lll}\text { II } \quad \text { Background } & 11\end{array}$

A. Characteristics of nonlinear oscillators . . . . . . . . . . . . . . . 11

1. The van der Pol equation . . . . . . . . . . . . . . . 11

2. Entrainment and the Arnol'd tongue . . . . . . . . . . . . . 13

3. Periodic pulling . . . . . . . . . . . . . . . . . . 15

4. Modified equations for $\alpha$ and $M \ldots \ldots \ldots \ldots$ 
B. Experimental context . . . . . . . . . . . . . . . 20

1. Nonlinear electronic oscillator . . . . . . . . . . . . . . . 20

2. Temporal vs. spatiotemporal periodic pulling . . . . . . . . . . 21

3. Optically driven waves . . . . . . . . . . . . . . . 22

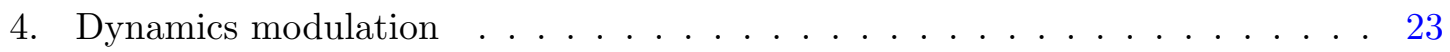

III Experimental Approach $\quad 27$

A. Experimental arrangement . . . . . . . . . . . . . . . 27

1. Neon glow discharge tube and circuit . . . . . . . . . . . . . . 27

2. Narrowband dye laser . . . . . . . . . . . . . . . . . 28

3. Controlling driving frequency . . . . . . . . . . . . . . . . . . 29

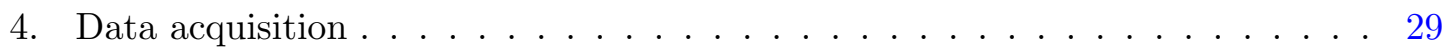

B. Diagnostic methods . . . . . . . . . . . . . . . . 30

1. Time series analysis . . . . . . . . . . . . . . . 30

2. Establishing the boundaries of the Arnol'd tongue . . . . . . . . . . . . . 31

C. Signatures of distinct behaviors . . . . . . . . . . . . . . 33

1. Temporal periodic pulling and entrainment . . . . . . . . . . 33

2. Spatiotemporal periodic pulling . . . . . . . . . . . . 34

3. Dynamics modulation $\ldots \ldots \ldots \ldots$

IV Experimental Results $\quad 37$

A. Modes in the discharge . . . . . . . . . . . . . . . . 38

B. Arnol'd tongue boundaries . . . . . . . . . . . . . . . . . . 39

1. Arnol'd tongue diagrams where one mode dominates . . . . . . . . . . . . 39

2. Arnol'd tongue boundaries near mode transitions . . . . . . . . . . . . 40

3. Occurrence of dynamics modulation . . . . . . . . . . . . . . . 40

C. Upward dynamics modulation . . . . . . . . . . . . . . . . . . . 41

1. Time series data versus driving frequency . . . . . . . . . . . . . 41

2. Extracting $\alpha$ and $M$ from time series data . . . . . . . . . . 42

3. Results of the $\alpha$ and $M$ calculations . . . . . . . . . . . 44

4. Detailed examination of mode behavior . . . . . . . . . . . . . 45 
D. Downward dynamics modulation . . . . . . . . . . . . . . . 46

$\begin{array}{lll}\text { V Modeling } & 49\end{array}$

A. Description of the model . . . . . . . . . . . . . . . . . . . 49

1. Requirements . . . . . . . . . . . . . . . . . . 49

2. Coupled van der Pol equations . . . . . . . . . . . . . . . 50

3. Step-by-time-step solution . . . . . . . . . . . . . . . . . . . 51

4. Handling mode transitions . . . . . . . . . . . . . . . . . 52

B. Identifying experimental parameters . . . . . . . . . . . . . . . . 53

1. Successful examples . . . . . . . . . . . . . . . . . . 53

C. Characterizing parameters of the model . . . . . . . . . . . . . . 55

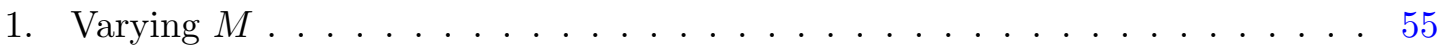

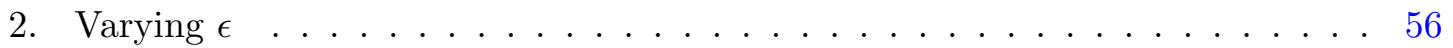

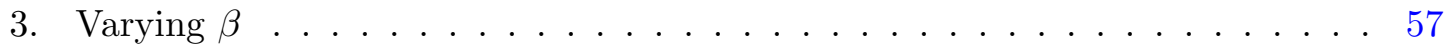

4. Varying the coupling parameters, $\gamma_{1}$ and $\gamma_{2} \ldots \ldots \ldots \ldots$

D. Dynamics modulation modeling summary . . . . . . . . . . . . . 58

1. Upward dynamics modulation . . . . . . . . . . . . . . . 58

2. The downward case . . . . . . . . . . . . . . . . 60

3. Summary of modeling results . . . . . . . . . . . . . . 61

$\begin{array}{llc}\text { VI Discussion } & 63\end{array}$

A. Evaluation of the mechanism . . . . . . . . . . . . . . . 64

1. Relative reduction in $M$ during the higher-frequency, temporal portion of the modulation ............................ 64

2. Subdominant mode alternation between laser entrainment and temporal periodic pulling ............................ 64

3. The dynamics phase diagram $\ldots \ldots \ldots \ldots \ldots$

4. $M$ variation is consistent with upper-mode amplitude change . . . . . . . 65

5. The upward mode transition occurs when sufficiently close . . . . . . . . 66

6. The upward mode transition occurs when the lower mode is out of phase with

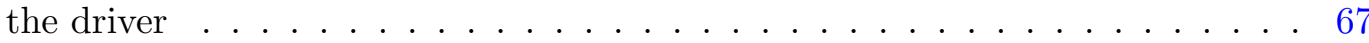


7. The downward mode transition . . . . . . . . . . . . 68

8. Reverse evolution of temporal periodic pulling . . . . . . . . . . . . 69

9. Verdict on the mechanism . . . . . . . . . . . . . . . 69

B. Interpretation of the "extra" beat frequency $\ldots \ldots \ldots \ldots$

1. The driven case of Chapter IV . . . . . . . . . . . . . . . . . 70

2. General implications . . . . . . . . . . . . . . . . . 71

C. Discussion of the model . . . . . . . . . . . . . . . . . . . 72

1. Shortcomings of the model . . . . . . . . . . . . . . . . . . 72

2. Successful aspects of the model . . . . . . . . . . . . . . 73

D. The downward modulation case . . . . . . . . . . . . . . 73

$\begin{array}{lll}\text { VII Conclusions } & 75\end{array}$

$\begin{array}{ll}\text { Appendices } & 77\end{array}$

$\begin{array}{lll}\text { A. } & \text { Derivation of the phase evolution equation } & 79\end{array}$

B. Computer code $\quad 81$

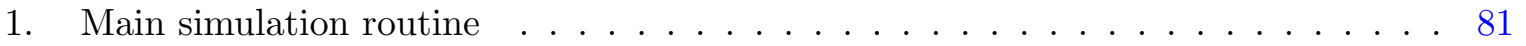

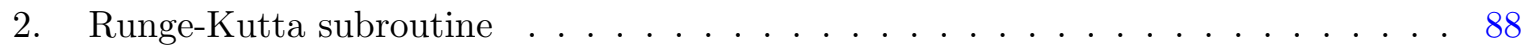

$\begin{array}{ll}\text { References } & 93\end{array}$

$\begin{array}{lr}\text { Tables } & 99\end{array}$

$\begin{array}{ll}\text { Figures } & 103\end{array}$ 


\section{List of Tables}

1.1 The Novák constants for ionization waves in low temperature plasmas. The first list comes from Novák (1960) and reflects the original empirical values. The second list comes from a recent review [Kolobov (2006)], and includes theoretical considerations. 101

4.1 Approximate frequency ranges of the glow discharge modes. Frequency range is the difference between the high and low extremes of each mode. Mode frequency separation is measured from the low extreme of each mode to the low extreme of the mode below. . . . . . . . . . . . . . . . . . . . . . . 101

4.2 Summary of the main dynamics modulation time series data sets reported in Chapter

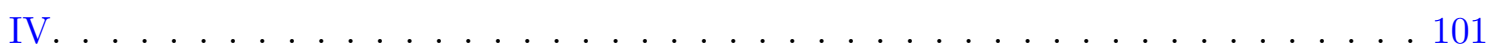

6.1 Comparison of temporal beat frequency $\Omega_{T} / 2 \pi$, taken from the power spectrum, to the direct measurement of periodic dynamics modulation frequency $f_{D M}$ taken from periods on instantaneous frequency series. Agreement is excellent. . . . . . . . . . . 102 
Page is intentionally blank 


\section{List of Figures}

1.1 A schematic diagram of the named parts of a glow discharge column. Depending on the experimental parameters of the discharge tube and its circuit elements, some of the parts may not be visible and their relative sizes may vary. [Figure created by Ian Tresman. Used under Creative Commons Attribution 2.5.] . . . . . . . . . . . . 104

1.2 $E \lambda$ versus discharge current. Different ionization wave types can be unambiguously distinguished using their Novák constants. Note that all four types do not occur at all pressures, which is why no $r$ waves appear in this plot, and that striations at higher currents do not follow the Novák rule. [From Garscadden (1978).] . . . . . . . 105

1.3 Ionization wave map. The regions in which the four kinds of ionization waves exist can be mapped against discharge current and pressure. Note the homogenous column in which no ionization waves occur. [From Garscadden (1978).] . . . . . . . . . . . 106

1.4 Possible wave states in the positive column of a neon discharge tube. Note the region labeled striated. Work in this thesis takes place at $p R \sim 1.5$ Torr $\mathrm{cm}$ and $I / r \sim 10^{-2} \mathrm{~A} / \mathrm{cm}$, near the I on the figure. The Pupp limit is indicated by curve labeled 1. [From Kolobov (2006).] . . . . . . . . . . . . . . . 107

1.5 Striation frequency versus current in $\mathrm{Ne}$ at $p R=1$ Torr $\mathrm{cm}$. The work in this thesis was done at $p R=1.5$ Torr $\mathrm{cm}$, so this figure is representative of ionization wave behavior in the experiment. The different shapes represent different discharge tube radii, with $\bullet(\boldsymbol{\Lambda})$ representing $R=1.5(2.9) \mathrm{cm}$. The hollow shapes represent artificially driven waves. [From Kolobov (2006).] . . . . . . . . . . . . . . 108 
2.1 Schematic diagram of an Arnol'd tongue. This figure can be reproduced by calculating $\alpha$ using Eq. 2.6 and setting $2 a=15$. The dark inverted triangle (arrow) is the region where $\alpha>1$, the Arnol'd tongue. Periodic pulling is strongest very near the Arnol'd tongue, as indicated. [From Klinger et al. (1995).] . . . . . . . . . . . . . 109

2.2 The time series behavior for three cases of driving frequency. Using the van der Pol equation (2.1) with $\epsilon=0.4, \beta=0.1, \omega_{0} / 2 \pi=160 \mathrm{~Hz}$ and $E=1.5, \omega_{i} / \omega_{0}$ was selected to demonstrate weak periodic pulling (top, $\omega_{i} / \omega_{0}=1.2157$ ), strong periodic pulling (center, $\left.\omega_{i} / \omega_{0}=1.1195\right)$, and entrainment (bottom, $\omega_{i} / \omega_{0}=1.05$ ). Parameters were chosen to approximately reproduce Figures 3-5 in Klinger et al.

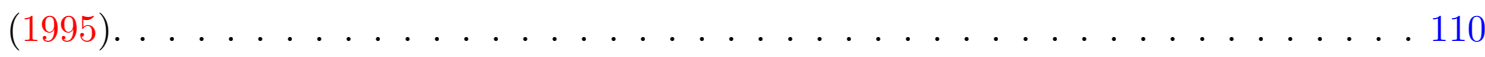

2.3 FFT amplitude of the time series shown in Figure 2.2. Using the van der Pol equation (2.1) with $\epsilon=0.4, \beta=0.1, \omega_{0} / 2 \pi=160 \mathrm{~Hz}$ and $E=1.5, \omega_{i} / \omega_{0}$ was selected to demonstrate weak periodic pulling (left panel, $\omega_{i} / \omega_{0}=1.2157$ ), strong periodic pulling (center panel, $\omega_{i} / \omega_{0}=1.1195$ ), and entrainment (right panel, $\omega_{i} / \omega_{0}=1.05$ ). Parameters were chosen to approximately reproduce Figures 3-5 in Klinger et al.

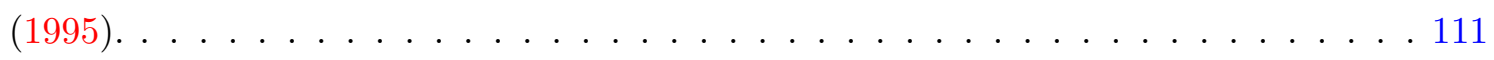

2.4 Four cases of $\alpha$, showing power spectra sideband structure (a) and phase development (b) as a function of time. [From Klinger et al. (1995).] . . . . . . . . . . . . 112

2.5 Arnol'd tongue cross section. Figure from Koepke and Hartley (1991), using data from a UJT circuit. . . . . . . . . . . . . . . . . . . . 113

2.6 Instantaneous frequency of the time series shown in Figure 2.2. Using the van der Pol equation (2.1) with $\epsilon=0.4, \beta=0.1, \omega_{0} / 2 \pi=160 \mathrm{~Hz}$ and $E=1.5, \omega_{i} / \omega_{0}$ was selected to demonstrate weak periodic pulling (top, $\omega_{i} / \omega_{0}=1.2157$ ), strong periodic pulling (center, $\left.\omega_{i} / \omega_{0}=1.1195\right)$, and entrainment (bottom, $\left.\omega_{i} / \omega_{0}=1.05\right) . \ldots 114$

2.7 Time series and FFT as driving frequency is adjusted from below the natural frequency (a through $\mathrm{f}$ ) to above the natural frequency (i through $\mathrm{n}$ ). Periodic pulling is evident on both sides of entrainment ( $g$ and $h$ ). Data from a UJT circuit. Figure from Koepke and Hartley (1991). . . . . . . . . . . . . . . . . . . 115 
2.8 Temporal periodic pulling versus spatio-temporal periodic pulling. Many examples of temporal periodic pulling (a1 and a2, and points on the curve) fit nicely on a predicted curve of beat frequency versus driving frequency. Koepke et al. (1996) estimate that the spatio-temporal case (b) has a similar driving strength to the temporal cases, but does so from a greater frequency separation. Therefore, it has a much larger effective driving amplitude $M$ based on Eq. (2.8). Figure from Koepke et al. (1996), using data from a neon glow discharge. . . . . . . . . . . 116

2.9 Dynamics modulation. The instantaneous frequency clearly and periodically hops up to the $1.75 \mathrm{kHz}$ mode and back down to the $1.5 \mathrm{kHz}$ mode. Figure from Weltmann et al. (2000), using data from a neon glow discharge. . . . . . . . . . . . . 117

2.10 Dynamics phase diagram for a multimode oscillator. The $\triangle$ represents a case of temporal periodic pulling. The $\boldsymbol{\nabla}$ represents spatiotemporal periodic pulling with no dynamics modulation. The represents entrainment of the higher mode, with no dynamics modulation. The $\bullet$ and $\mathbf{\square}$ cases represent two cases of dynamics modulation when combined with their counterparts, $\circ$ and the $\square$. See text for a complete explanation. From Weltmann et al. (2000). . . . . . . . . . . . . 118

3.1 The experimental arrangement. The cathode of the discharge tube is on the left, and the anode is on the right. A $10 \Omega$ resistor (not pictured) was placed between the anode and ground, and the voltage across this resistor was used to measure the undriven modes for Figures 4.1 and 4.3. More description of the experimental arrangement can be found in the text. . . . . . . . . . . . . 119

3.2 Iodine cell calibration. Output of iodine cell (o) with fit (dashed line) to nearby lines from iodine atlas, which establishes the wavelength range. The outer triangles on the axis represent the approximate wavelength extremes at which the laser just begins to affect the discharge. Between the inner pair of triangles, the laser can entrain any driving frequency in the discharge. The two five-pointed stars have been placed at representative wavelengths where dynamics modulation has been observed. . . . . . 120 
3.3 Optics (a) and electronics (b) of the photodiode circuit. Light would enter from left to right, and pass through two $50 \mathrm{~mm}$ focal length lenses. Between the lenses, there is a $640.1 \mathrm{~nm}$ (4 nm bandwidth) filter. More details about the design appear in the

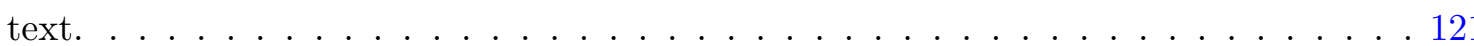

3.4 Identifying the edge of an Arnol'd tongue from the power spectra. The $y$-axis shows the logarithm of the magnitude of the FFT. Mode frequency is around $1440 \mathrm{~Hz}$. The driving frequency is shown on the left side of each of the power spectra, and it is driving the mode from below. Peaks are visible at the driving frequency in each panel. Periodic pulling increases going down the figure until, finally, in the bottom panel, the oscillation is entrained to the driving frequency. . . . . . . . . . . . 122

3.5 Spectrum of temporal periodic pulling. The discharge current was set to $7.87 \mathrm{~mA}$. The driving frequency (here at $1522 \mathrm{~Hz}$ ) is above the undriven oscillation frequency $(1466 \mathrm{~Hz})$.

3.6 Instantaneous frequency series for temporal periodic pulling. The instantaneous frequency (bottom) and the corresponding time series is shown (top) is shown. The instantaneous frequency has a clear beat shown by arcs in a concave-downward orientation. While the oscillations occur so fast that they darken the entire region, it is clear that their overall amplitude is modulated along with the frequency. This figure displays the same data as Figure 3.5, so the driving frequency is aobve the dominant mode frequency. . . . . . . . . . . . . . . . . . . . 124

3.7 Temporal periodic pulling from each side. The case of Figures 3.5 and 3.6 (bottom), which had driving frequency $(1522 \mathrm{~Hz})$ above the mode frequency $(1466 \mathrm{~Hz})$, is shown alongside another case (top), with the driving frequency $(1430 \mathrm{~Hz}$ ) below the mode frequency $(1470 \mathrm{~Hz})$. Here the instantaneous frequency beat period arc appears concave up. Also clear from this figure is that the sidebands appear on the opposite side of the oscillation from the driving frequency. $\Omega$, determined from sideband separation, is $35.8 \mathrm{~Hz}$ (top) and $41.6 \mathrm{~Hz}$ (bottom) . . . . . . . . 125 
3.8 Signature of spatiotemporal periodic pulling. In the top half of the figure, the power spectrum is shown. The instantaneous frequency is shown on the bottom. The periodic pulling, evident by the range of the frequency oscillation, is much stronger than it was in the case of Figures 3.5 to $3.7 \ldots \ldots \ldots \ldots$

3.9 Close up of spatiotemporal time series and instantaneous frequency series. . . . . . 127

3.10 Signature of dynamics modulation. The distinct "hairy" spectrum (top) and the visible mode changes in the instantaneous frequency series (bottom). The undriven frequency is $1450 \mathrm{~Hz}$, and the driving frequency was set to $1704 \mathrm{~Hz}$. . . . . . . . 128

3.11 Instantaneous frequency of undriven mode transitions. [From Weltmann et al. (2000)].129

4.1 Ionization wave modes as a function of discharge current. Vertical lines indicate hysteresis. The right-hand (left-hand) line of each pair represents the discharge current for which the mode changes as the current is increasing (decreasing). The triangles indicate mode frequencies measured between increasing $(\boldsymbol{\Lambda})$ or decreasing $(\boldsymbol{\nabla})$ the current. . . . . . . . . . . . . . . . . . . . . . . . . . . .

4.2 An example of the wave spectrum with no driving force. The $y$-axis shows the logarithm of the power spectrum. . . . . . . . . . . . . . 131

4.3 Close-up views of four of the wave modes shown in Figure 4.1. Mode frequencies are not constant as discharge current changes. . . . . . . . . . . . . . . 132

4.4 Arnold tongue for $I_{D}=9.10 \mathrm{~mA}$. The long feature in the center is the $f 7$ mode. $f 6$ and $f 8$ appear to the left and right, respectively. Since they are not natural modes at this discharge current, $f 6$ and $f 8$ are shorter in the $y$-axis direction. With the laser off, the natural frequency is $\sim 1450 \mathrm{~Hz} \ldots \ldots \ldots \ldots$

4.5 Arnold tongue for $I_{D}=11.37 \mathrm{~mA}$. The long feature in the center is the $f 8$ mode. $f 7$ and $f 9$ appear to the left and right, respectively. With the laser off, the natural frequency is $\sim 1721 \mathrm{~Hz} \ldots \ldots \ldots \ldots \ldots \ldots \ldots$

4.6 Combined view of data from Figures 4.4 and 4.5 showing how the two data sets overlap one another. The currents were $9.10 \mathrm{~mA}(\bullet)$ and $11.37 \mathrm{~mA}(\mathbf{\square}) . . . . . .135$ 
4.7 Arnol'd tongue boundaries from $I_{D}=8.23 \mathrm{~mA}$ and $I_{D}=10.45 \mathrm{~mA}$, near the mode transition boundaries on either side of the $f 7$ mode. These two boundary maps result from adjusting the discharge current downward $(\boldsymbol{\Delta})$ or upward $(\bullet)$ from that shown in Figure 4.4. The downward points of the Arnol'd tongue occur at $1212 \mathrm{~Hz}$, at $1462 \mathrm{~Hz}$ (lower current) and at $1447 \mathrm{~Hz}$ (higher current), and $1726 \mathrm{~Hz}$. These values are consistent with Figure $4.3 \ldots \ldots \ldots$. . . . . . . . . . . . . . . . . .

4.8 The occurrence of dynamics modulation ( $\mathbf{\Delta})$ relative to the Arnol'd tongue of the $f 7$ mode. The cluster of points on the right (left) side result in upward (downward) dynamics modulation. On the right, where two triangles appear side-by-side at the same $y$-axis location, dynamics modulation occurs throughout the interval. Note that all cases of dynamics modulation ended in entrainment, so the small offset between the tip of the $f 8$ mode and the series of triangles is not significant. . . . . 137

4.9 The occurrence of dynamics modulation ( $\mathbf{\Delta})$ relative to the Arnol'd tongue of the f8 mode. The cluster of points on the right (left) side result in upward (downward) dynamics modulation. On the right, where two triangles appear side-by-side at the same $y$-axis location, dynamics modulation occurs throughout the interval. . . . . 138

4.10 A sequence of time series for upward dynamics modulation as a function of driving frequency. The $y$-axis of each plot shows the signal amplitude from the photodiode. Beginning at the top, the driving frequency $\left(f_{i}\right)$ is 1681 (a), 1688 (b), 1696 (c), 1704 (d), 1710 (e) and 1713 (f) Hz. The discharge current was set to $9.10 \mathrm{~mA}$. Entrainment occurred at $1712 \mathrm{~Hz}$. . . . . . . . . . . . . . . . 139

4.11 A sequence of instantaneous frequency series for upward dynamics modulation as a function of driving frequency. The $y$-axis of each plot shows the instantaneous frequency in $\mathrm{Hz}$ for the time series shown in Figure 4.10. Beginning at the top, the driving frequency $\left(f_{i}\right)$ is 1681 (a), 1688 (b), 1696 (c), 1704 (d), 1710 (e) and 1713 (f) $\mathrm{Hz}$. The modulation is taking place between the $f 7$ and $f 8$ modes. The discharge current was set to $9.10 \mathrm{~mA}$. Entrainment occurred at $1712 \mathrm{~Hz}$. . . . . . . . . . . 140 
4.12 A sequence of power spectra for upward dynamics modulation as a function of driving frequency. The power spectra corresponding to the time series shown in Figure 4.10. Beginning at the top, the driving frequency $\left(f_{i}\right)$ is 1681 (a), 1688 (b), 1696 (c), 1704 (d), 1710 (e) and 1713 (f) Hz. The discharge current was set to $9.10 \mathrm{~mA}$. Entrainment occurred at $1712 \mathrm{~Hz}$. . . . . . . . . . . . . . . . . 141

4.13 A wider view, including low frequencies, of the two spectra shown in Figure 3.7. There is a dominant low frequency peak that corresponds to the spacing between the sidebands on the main feature. This feature can be used to identify the modified beat frequency $\Omega$, which is then used to find the entrainment parameter $\alpha$ for temporal periodic pulling when the method described in Chapter III is obscured by the activity of dynamics modulation. . . . . . . . . . . . . . . . . . 142

4.14 An example that shows how to calculate the $\Omega$ frequency for the two states of dynamics modulation. The top panel shows an instantaneous frequency series, with horizontal lines drawn at 1350 and $1700 \mathrm{~Hz}\left(=f_{i}\right)$. The beat frequency was calculated using the instantaneous frequency routine on this series. Instead of examining zero crossings, crossings of the two lines were used. The beat frequency modulated between two values, as can be seen in the lower panel. The dashed lines represent the median values of the lower $(\bullet, 224.3 \mathrm{~Hz})$ and higher $(\mathbf{\square}, 273.1 \mathrm{~Hz})$ measured beats. The discharge current was set to $9.10 \mathrm{~mA}$. Entrainment occurred at $1713 \mathrm{~Hz} .143$

4.15 Reproducible values for $\Omega_{S T}$ and $\Omega_{T}$ obtained from dynamics modulation data sets. These come from the sequence of time series highlighted in Figures 4.10 through 4.12, as well as a second series taken on a different day, depicting upward modulation between $f 7$ and $f 8$. In the top (bottom) panel, the spatiotemporal (temporal) beat frequencies are calculated for two data sets on two different days, as a function of entrainment frequency minus driving frequency. The dotted line shows the conventional beat frequency $\left(\Omega_{0}\right)$ and the dashed line shows the reference beat frequency $\left(\Omega^{\prime}\right)$. 
4.16 The results of $\alpha$ and $M$ calculations from the sequence of time series highlighted in Figures 4.10 through 4.12, as well as a second series taken on a different day, depicting upward modulation between $f 7$ and $f 8$. On the left is the spatiotemporal case in which the entrained upper mode drives the lower mode. On the right, the temporal case in which the driver drives the upper mode. The $x$-axis displays entrainment frequency minus driving frequency. The solid lines to the right (and near the top of the temporal $\alpha$ subplot) indicate the entrainment boundary for the $f 8$ mode. The dotted line in the top-left panel indicates the entrainment boundary for the $f 7$ mode, which is never approached. Note that $\alpha_{T}$ approaches one just as the upper mode becomes entrained. The discharge current was set to $9.10 \mathrm{~mA}$. . . . . . . . . . 145

4.17 The results of $\alpha$ and $M$ calculations from another sequence of time series taken on a different day and at a different discharge current. In this case the data depicts upward modulation between $f 8$ and $f 9$. Again, on the left is the spatiotemporal case in which the entrained upper mode drives the lower mode. On the right, the temporal case in which the driver drives the upper mode. The solid lines to the right (and near the top of the temporal $\alpha$ subplot) indicate the entrainment boundary for the $f 9$ mode. The dotted line in the top-left panel indicates the entrainment boundary for the $f 8$ mode, which is never approached. Again, $\alpha_{T}$ approaches one just as the upper mode becomes entrained, though not quite as convincingly. The discharge current was set to $11.37 \mathrm{~mA}$. Entrainment occurred at $2013 \mathrm{~Hz}$. . . . . . . 146

4.18 The ratio of $M_{S T}$ to $M_{T}$ for the two data sets of Figure 4.16. The ratio steadily increases as the entrainment boundary for the $f 8$ mode is approached. The discharge current was set to $9.10 \mathrm{~mA}$. Entrainment occurred at $1712(\bullet)$ and $1713(\boldsymbol{\square}) \mathrm{Hz}$ on the two days, with the difference being due to slight variation in laser power and/or

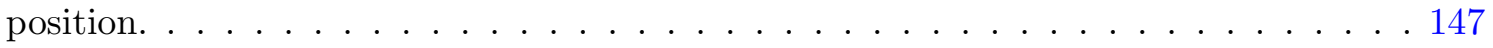

4.19 The ratio of $M_{S T}$ to $M_{T}$ for the data of Figure 4.17. Again, the ratio steadily increases as entrainment boundary for the $f 9$ mode is approached. The discharge current was set to $11.37 \mathrm{~mA}$. Entrainment occurred at $2013 \mathrm{~Hz}$. . . . . . . . . . . . 148 
4.20 Time series close-up of lower mode during periodic dynamics modulation. Along with the upper, time series trace, the square-wave driving frequency is shown scaled and offset. This segment of the modulation begins with a discontinuity in the time series at $t=0.2270 \mathrm{~s}$, and ends at $t=0.2507 \mathrm{~s}$. During the time spent in the lower mode, the amplitude beats with a period of $0.00457 \mathrm{~s}$. The driving frequency, $f_{i}$, was set to $1690 \mathrm{~Hz} \ldots \ldots \ldots \ldots$. . . . . . . . . . . . . . . . . 149

4.21 Time series close-up of higher mode during periodic dynamics modulation. This segment of the modulation begins when the time series and driver quickly change from out-of-phase to in-phase at $t=0.0124 \mathrm{~s}$, and ends with the opposite change at $t=0.0329 \mathrm{~s}$. During the time spent in the higher mode, the amplitude beats with a period of $0.00430 \mathrm{~s}$. The driving frequency, $f_{i}$, was set to $1690 \mathrm{~Hz}$. . . . . . . 150

4.22 Time series close-up of lower mode during periodic dynamics modulation. The beginning of the lower frequency part of the cycle begins when the phase of the luminosity signal changes quickly at $t=0.2842 \mathrm{~s}$, and ends at $t=0.3071 \mathrm{~s}$. The signal almost snaps out of its lower mode at $t=0.3031 \mathrm{~s}$, but appears to remain for one more beat. During the time spent in the lower mode, the amplitude beats with a period of $0.00483 \mathrm{~s}$. The driving frequency, $f_{i}$, was set to $1700 \mathrm{~Hz} . \ldots \ldots \ldots 1$

4.23 Time series close-up of higher mode during periodic dynamics modulation. The beginning of the higher frequency part of the cycle begins when the phase of the luminosity signal changes quickly at $t=0.0108 \mathrm{~s}$, and ends at $t=0.0400 \mathrm{~s}$. During the time spent in the higher mode, the amplitude does not beat in as obvious a manner as the example shown in Figure 4.21 for a lower driving frequency, but the instantaneous frequency shows seven beat periods. The driving frequency, $f_{i}$, was

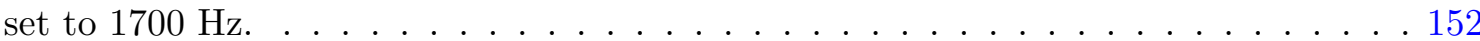

4.24 A representative example of downward dynamics modulation. The time series (top), instantaneous frequency (center) and power spectrum (bottom) of downward dynamics modulation are shown here. The driving frequency $\left(f_{i}\right)$ was $1200 \mathrm{~Hz}$. The modulation is taking place between the $f 7$ and $f 6$ modes. The discharge current

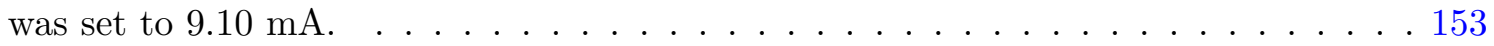


4.25 A sequence of instantaneous frequency series for downward dynamics modulation as a function of driving frequency. The $y$-axis of each plot shows the instantaneous frequency in Hz. Beginning at the top, the driving frequency $\left(f_{i}\right)$ is 1385 (a), 1395 (b), 1403 (c), 1405 (d), 1406 (e) and 1409 (f) Hz. The modulation is taking place between the $f 7$ and $f 6$ modes. Panels (a) through (e) represent aperiodic downward dynamics modulation, and panel (f) represents entrainment of the $f 6$ mode. The discharge current was set to $9.10 \mathrm{~mA}$. Entrainment occurred at $1409 \mathrm{~Hz}$. . . . . . . 154

4.26 A sequence of power spectra for downward dynamics modulation as a function of driving frequency. The $y$-axis of each plot shows the instantaneous frequency in Hz. Beginning at the top, the driving frequency $\left(f_{i}\right)$ is 1385 (a), 1395 (b), 1403 (c), 1405 (d), 1406 (e) and 1409 (f) Hz. The modulation is taking place between the $f 7$ and $f 6$ modes. Panels (a) through (e) represent aperiodic downward dynamics modulation, and panel (f) represents entrainment of the $f 6$ mode. The discharge current was set to $9.10 \mathrm{~mA}$. Entrainment occurred at $1409 \mathrm{~Hz}$. . . . . . . . . . . . . 155

4.27 A sequence of instantaneous frequency series for downward dynamics modulation as a function of driving frequency. The $y$-axis of each plot shows the instantaneous frequency in Hz. Beginning at the top, the driving frequency $\left(f_{i}\right)$ is 1420 (a), 1423 (b), 1426 (c), 1429 (d), 1432 (e) and 1435 (f) Hz. The modulation is taking place between the $f 7$ and $f 6$ modes. Panels (a) through (c) represent downward dynamics modulation. Panels (d) and (e) represent temporal periodic pulling of the $f 6$ mode only and panel (f) represents entrainment. The discharge current was set to 9.10 mA. Entrainment occurred at $1435 \mathrm{~Hz} \ldots \ldots \ldots \ldots$. . . . . . . . . 156

4.28 A sequence of power spectra for downward dynamics modulation as a function of driving frequency. The $y$-axis of each plot shows the instantaneous frequency in Hz. Beginning at the top, the driving frequency $\left(f_{i}\right)$ is 1420 (a), 1423 (b), 1426 (c), 1429 (d), 1432 (e) and 1435 (f) Hz. The modulation is taking place between the $f 7$ and f6 modes. Panels (a) through (c) represent downward dynamics modulation. Panels (d) and (e) represent temporal periodic pulling of the $f 6$ mode only and panel (f) represents entrainment. The discharge current was set to $9.10 \mathrm{~mA}$. Entrainment occurred at $1435 \mathrm{~Hz} \ldots \ldots \ldots \ldots \ldots \ldots \ldots \ldots$ 
5.1 A good example of temporal periodic pulling in the model. Here, $\epsilon_{1}=0.1, \beta_{1}=1$, and $M=0.03$. The coupling parameters were set at $\gamma_{1}=0.025$ and $\gamma_{2}=0.22$. $\omega_{1}=2 \pi \times 1450$ and $\omega_{2}=2 \pi \times 1740$, the values from the experimental data. For this one case, $\epsilon_{2}=\beta_{2}=M_{2}=0$, to keep the second mode separate. $f_{i}$ was 1500 (a), 1486 (b), 1472 (c), 1458 (d) 1444 (e), and 1430 (f) Hz. Panel (f) represents entrainment rather than periodic pulling. The qualitative behavior of the model (Figure 5.1) is very similar to that of the experiment seen in Figure $3.4 \ldots \ldots \ldots$

5.2 Simulated dynamics modulation. Here, $\epsilon=0.1$ and $\beta=1$. The coupling parameters were set at $\gamma_{1}=0.025$ and $\gamma_{2}=0.22$ and the mode frequencies were set at $\omega_{1}=$ $2 \pi \times 1450$ and $\omega_{2}=2 \pi \times 1740$. The driving frequency, $f_{i}$, was $1705 \mathrm{~Hz}$. The panels, in order from top to bottom, show the time series, the instantaneous frequency, the value of $M$ used in the simulation in response to the detected mode, and the power

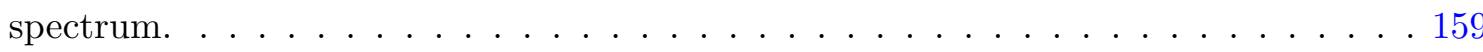

5.3 Simulated spatiotemporal periodic pulling. $f_{i}$ was set to $1675 \mathrm{~Hz}$. Other inputs were the same as Figure 5.2. The result is an excellent example of spatiotemporal periodic pulling from above. This is shown by the concave-downward instantaneous frequency plot (bottom) which corresponds to the time series presented above it. . . 160

5.4 Power spectra as a function of $M . M$ was set to 0.0028 (a), 0.0091 (b), 0.0295 (c), 0.0955 (d), 0.3090 (e), and 1.000 (f). The remaining parameters were $\epsilon=0.1, \beta=1$, and coupling parameters $\gamma_{1}=0.025$ and $\gamma_{2}=0.22$. The mode frequencies were set at $\omega_{1}=2 \pi \times 1450 \mathrm{~Hz}$ and $\omega_{2}=2 \pi \times 1740 \mathrm{~Hz}$. The driving frequency was set to

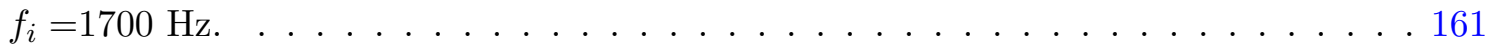


5.5 Instantaneous frequency trend as a function of $M$. The solid line traces the median instantaneous frequency value as a function of normalized driving amplitude $M$. Dotted lines above and below represent extreme values. The mode frequencies were set at $\omega_{1}=2 \pi \times 1450 \mathrm{~Hz}$ and $\omega_{2}=2 \pi \times 1740 \mathrm{~Hz}$. The driving frequency was set to $f_{i}=1700 \mathrm{~Hz}$. In the limit of low driving amplitudes, the median instantaneous frequency stays near $1450 \mathrm{~Hz}$. In the limit of high driving amplitudes, the median instantaneous frequency equals the driving frequency. The dashed lines represent $M_{H}$ (left) and $M_{L}=2 M_{H}$ (right) used in the model. Parameters were $\epsilon=0.1$, $\beta=1$, and coupling parameters $\gamma_{1}=0.025$ and $\gamma_{2}=0.22 \ldots \ldots \ldots 2$

5.6 Power spectra as a function of $\epsilon$. $\epsilon$ was set to 0.0100 (a), 0.0251 (b), 0.0631 (c), 0.1585 (d), 0.3981 (e) and 1.000 (f). Parameters were $M=0.03, \beta=1$, and coupling parameters $\gamma_{1}=0.025$ and $\gamma_{2}=0.22$. The mode frequencies were set at $\omega_{1}=2 \pi \times 1450 \mathrm{~Hz}$ and $\omega_{2}=2 \pi \times 1740 \mathrm{~Hz}$. The driving frequency was set to $f_{i}=1700$ Hz. . . . . . . . . . . . . . . . . . . . 163

5.7 Power spectra as a function of $\beta$. $\beta$ was set to 0.0100 (a), 0.0251 (b), 0.0631 (c), 0.1585 (d), 0.3981 (e) and 1.000 (f). Parameters were $M=0.03, \epsilon=0.1$, and coupling parameters $\gamma_{1}=0.025$ and $\gamma_{2}=0.22$. The mode frequencies were set at $\omega_{1}=2 \pi \times 1450 \mathrm{~Hz}$ and $\omega_{2}=2 \pi \times 1740 \mathrm{~Hz}$. The driving frequency was set to $f_{i}=1700$

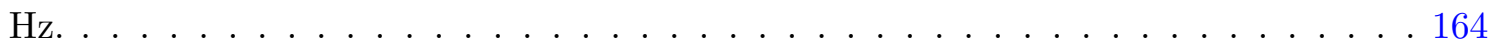

5.8 Dependence on coupling $\gamma$, with $\gamma_{1}=\gamma_{2}=\gamma$. The values used for $\gamma$ in this case are 0.001 (a), 0.0608 (b), 0.1206 (c), 0.1804 (d), 0.2402 (e), and 0.3000 (f). Parameters were $M=0.03, \epsilon=0.1$, and $\beta=1$. The mode frequencies were set at $\omega_{1}=$ $2 \pi \times 1450 \mathrm{~Hz}$ and $\omega_{2}=2 \pi \times 1740 \mathrm{~Hz}$. The driving frequency was set to $f_{i}=1700 \mathrm{~Hz} .165$

5.9 Dependence on coupling $\gamma_{2}$, with $\gamma_{1}=0.025$. $\gamma_{2}$ was set to 0.0003 (a), 0.0602 (b), 0.1202 (c), 0.1801 (d), 0.2401 (e), and 0.3000 (f). This corresponds to $\gamma_{2} / \gamma_{1}$ ranging from 0.01 to 12.0. Parameters were $M=0.03, \epsilon=0.1$, and $\beta=1$. The mode frequencies were set at $\omega_{1}=2 \pi \times 1450 \mathrm{~Hz}$ and $\omega_{2}=2 \pi \times 1740 \mathrm{~Hz}$. The driving frequency was set to $f_{i}=1700 \mathrm{~Hz} \ldots \ldots \ldots \ldots$ 
5.10 Simulated dynamics modulation as a function of $f_{i}$, time series. The driving frequency, $f_{i}$, was 1685 (a), 1690 (b), 1700 (c), 1705 (d), 1715 (e), and 1725 (f). Other parameters were $M$ toggling between 0.02 (when high mode detected) and 0.04 (when low mode detected), $\epsilon=0.1$, and $\beta=1$. The coupling parameters were $\gamma_{1}=0.025$ and $\gamma_{2}=0.22$. The mode frequencies were set at $\omega_{1}=2 \pi \times 1450 \mathrm{~Hz}$ and

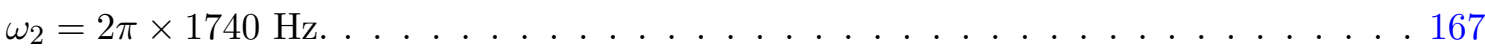

5.11 Simulated dynamics modulation as a function of $f_{i}$, instantaneous frequency. The driving frequency, $f_{i}$, was 1685 (a), 1690 (b), 1700 (c), 1705 (d), 1715 (e), and 1725 (f). Other parameters were $M$ toggling between 0.02 (when high mode detected) and 0.04 (when low mode detected), $\epsilon=0.1$, and $\beta=1$. The coupling parameters were $\gamma_{1}=0.025$ and $\gamma_{2}=0.22$. The mode frequencies were set at $\omega_{1}=2 \pi \times 1450 \mathrm{~Hz}$

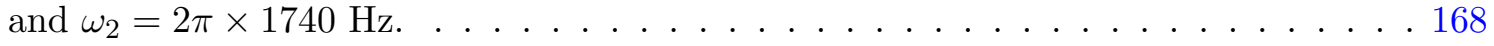

5.12 Simulated dynamics modulation as a function of $f_{i}$, power spectra. The driving frequency, $f_{i}$, was 1685 (a), 1690 (b), 1700 (c), 1705 (d), 1715 (e), and 1725 (f). Other parameters were $M$ toggling between 0.02 (when high mode detected) and 0.04 (when low mode detected), $\epsilon=0.1$, and $\beta=1$. The coupling parameters were $\gamma_{1}=0.025$ and $\gamma_{2}=0.22$. The mode frequencies were set at $\omega_{1}=2 \pi \times 1450 \mathrm{~Hz}$ and

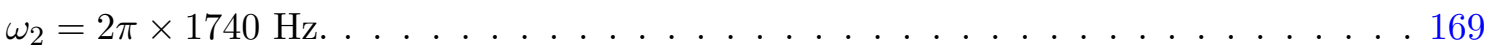

5.13 Symmetric Downward Modulation. This behavior was produced with the same $\epsilon$ $(0.1), \beta(1)$, and coupling parameters $\left(\gamma_{1}=0.025\right.$ and $\left.\gamma_{2}=0.22\right)$ as the upward case. $M$ was toggled between 0.28 (when the low mode was detected) and 0.56 (when the high mode was detected). The mode frequencies were set at $\omega_{1}=2 \pi \times 1703 \mathrm{~Hz}$ and $\omega_{2}=2 \pi \times 1455 \mathrm{~Hz}$. The driving frequency was set at $f_{i}=1460 \mathrm{~Hz}$. A similar symmetric case of downward modulation was not seen in the experiment. The panels, in order from top to bottom, show the time series, the instantaneous frequency, the value of $M$ used in the simulation in response to the detected mode, and the power

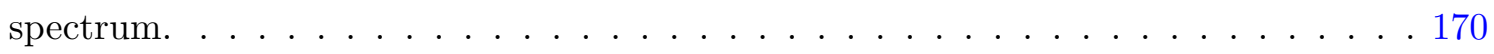


6.1 Dynamics modulation occurs at the temporal pulling beat frequency. This figure compares data from the temporal periodic pulling beat frequency (open), obtained from the low frequency peak on the power spectrum in the manner of Figure 4.14 with the periodic dynamics modulation frequency (filled), measured from the time series. This figure shows that the two frequencies are identical. Circles and squares represent data sets one and two respectively, modulating from $f 7$ to $f 8$. Triangles represent data set 3 , modulating from $f 8$ to $f 9 \ldots \ldots \ldots \ldots \ldots$

6.2 Asymmetry in reference beat frequency between two separated modes. The dotted lines represent the conventional beat frequency, $\Omega_{0}=\left|\omega_{i}-\omega_{0}\right|$. The reference beat frequency, $\Omega^{\prime}$ (calculated from Eq. (2.20)) is shown with the solid lines. For two modes separated significantly, this figure predicts that the beat frequency will be higher when the lower frequency pulls the higher frequency than when the opposite

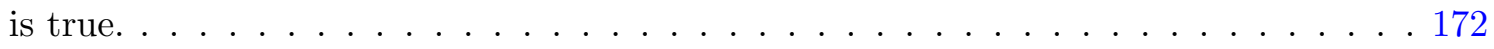

6.3 Upper beat frequency for low mode pulling high mode. This figure is the complementary figure to Figure 4.15 (top) for the high mode pulling the low mode. Measured beat frequencies are higher in this case. The dotted line is the conventional beat frequency $\Omega_{0}$ and dashed line is the reference beat frequency $\Omega^{\prime}$. In this case, since $\Omega^{\prime}$ is calculated based on the two mode frequencies only, it is not dependent on driving

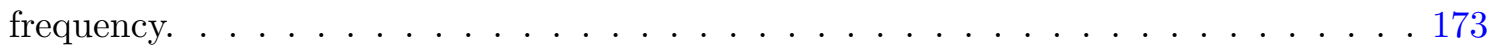

6.4 $\alpha$ and $M$ for the low mode pulling down on the high mode. This figure is the complementary figure to Figure 4.16 (left half) for the high mode pulling the low mode. Measured $\alpha$ and $M$ values are significantly higher in this case, reflecting the system's strong preference for the $f 7$ mode. The solid lines to the right indicate the entrainment boundary for the $f 8$ mode. The dotted line in the top panel indicates the entrainment boundary for the $f 7$ mode, which is never approached. . . . . . . 174 
6.5 Beat separation from the model. This figure shows that the model successfully predicts a shift in beat frequencies between the two parts of the dynamics modulation cycle. The driving frequency in this case was set to $1700 \mathrm{~Hz}$. The dashed lines in the bottom panel occur at the median frequencies of the lower $(\bullet, 258.6 \mathrm{~Hz})$ and higher $(\boldsymbol{\square}, 278.4 \mathrm{~Hz})$ beat frequencies. Compare this figure to the experimental case shown

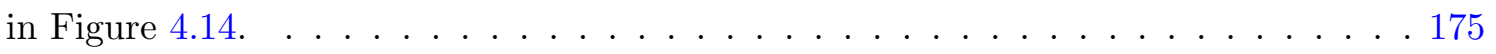

6.6 Multimode interaction may assist upward dynamics modulation but not the symmetric downward case. The bottom panel shows an observed downward dynamics modulation spectrum. For reference, light gray bars are placed at the observed mode frequencies for this discharge tube, and extended into the top panel. Medium gray rectangles are placed at driving frequencies used for dynamics modulation attempts as labeled. The black rectangles represent the first two expected sidebands opposite the driven mode. In the two successful cases, the first sideband overlaps an existing mode. For the symmetric downward modulation case, for which no dynamics modulation was observed, there is no overlap $($ circle $) \ldots \ldots \ldots \ldots$ 
Page is intentionally blank 


\section{Chapter I}

\section{Introduction}

The spontaneous development of striation layers (or ionization waves) in a glow discharge plasma was the earliest example of an instability in a laboratory plasma, with observations of the phenomenon first published in 1848 [Abria (1848)] and clear evidence that Michael Faraday had made his own notes on the phenomena even earlier [Kolobov (2006)]. Striations remain the most readily observed instability in a laboratory plasma [Pekárek (1968)] and even appear in poorly functioning fluorescent lamps. Despite the familiarity of the glow discharge, it is still a rich experimental environment. This chapter begins with a brief history of glow discharge physics followed by a brief description of the behavior of ionization waves. A brief section on nonlinear dynamics follows, with emphasis on the use of glow discharge tubes in this work. Finally, with the broad context in place, an overview of this thesis is presented.

\section{A. Glow discharge physics}

\section{A brief history of the plasma glow discharge}

The key technological innovation that enabled glow discharge tube research to thrive was the ability to seal platinum electrodes into glass tubes. This process, mastered by Heinrich Geissler in 1858, allowed these Geissler tubes to become common sources of entertainment. Soon, however, they were being studied scientifically as well. They were used as sources of light in an effort to understand the origin of spectral lines and as the source of the newly discovered cathode rays.

One of the giants of this early work was Sir William Crookes, who began with the simple idea 
of developing the technology into practical lighting. This effort failed to produce results promising enough to displace established chemical means of lighting, but research into cathode rays soon replaced these efforts [Brown (1978)]. Crookes, whose consulting had made him wealthy, was able to pursue his research using his own personal glassblower and his efforts did much to improve the technology of discharge tubes. Because of his efforts, discharge tubes optimized to produce cathode rays began to be referred to as Crookes Tubes.

A rich period of research followed the development of improved vacuum technology in 1880 [Brown (1978)]. For the half century that followed, the glow in glow discharge tubes was explored in great detail. Spectral lines were catalogued for many gases. While actual advances in physics were minimal and had to await the development of quantum physics and plasma physics, a variety of visual behaviors were catalogued and named [Brown (1978)].

The cathode ray question remained unanswered until the end of the nineteenth century, when J. J. Thomson correctly interpreted the rays as beams of electrons [Brown (1978)]. This discovery in discharge tube physics led to the Nobel prize for Thomson in 1906 and usually appears in introductory physics books.

In his 1927 letter to Nature, van der Pol used a "neon glow lamp" to study a peculiar relaxationoscillator circuit [van der Pol and van der Mark (1927)]. This use of a neon glow discharge began van der Pol's exploration of nonlinear oscillators, which led to his work with the now-famous van der Pol equation [see van der Pol (1934)], which will be described in Chapter II.

\section{Basic features of an undriven discharge tube}

The glow discharge itself consists of current passed through a gas between two electrodes, usually maintained at low pressure within a glass insulating tube [Ingold (1978)]. In Figure 1.1, a schematic diagram depicts the nomenclature of the typical glow discharge. This diagram shows the relative

positions of the Aston dark space, the cathode glow, the cathode dark space, the negative glow, the Faraday dark space, the positive column, the anode glow, and the anode dark space, in order from cathode to anode. Typically, the details of the metastable species are important to the dynamics of the discharge.

Depending on the physical dimensions of the discharge, the pressure of the enclosed gas, the shape of the electrodes, and the variety of gas, these features vary somewhat in relative size and 


\section{CHAPTER I. INTRODUCTION}

prominence. For example, in the present study, the Faraday dark space is very narrow, thus the light is continuous between the negative glow and the positive column with only a small narrowing of the glow at the Faraday dark space. For our purposes, these terms primarily label alternating areas of glowing plasma and relative darkness. The detailed physics that leads to the development of these recognizable features is reviewed in Ingold (1978).

A brief physical description distinguishing the positive column from the other features to the cathode side is supplied by Franklin (1976). Electrons, emitted from the cathode, undergo axial acceleration due to a strong local electric field and increase in energy to a value adequate to ionize the neutral gas. In the regions of the discharge between the cathode and the positive column, these electrons collide inelastically with increasing energy until an equilibrium is established between energy added from the electric field and energy lost to collisions. When the equilibrium is reached, it continues for most of the length of the discharge tube, in the region known as the positive column. This is the region of the plasma where the waves studied in this thesis are observed, and it has a relatively constant axial electric field aside from periodic perturbations with the striation wavelength.

\section{Ionization waves}

In discharge plasmas in general, a wide variety of waves have been observed across a wide parameter range. For example, waves have been associated with electron $\left(10^{8} \mathrm{~Hz}\right.$ to $\left.10^{10} \mathrm{~Hz}\right)$ and ion $\left(10^{5} \mathrm{~Hz}\right.$ to $\left.10^{7} \mathrm{~Hz}\right)$ oscillations, ion acoustic dispersion $\left(10^{3} \mathrm{~Hz}\right.$ to $10^{6} \mathrm{~Hz}$ in lower pressure discharges), and standing (0 Hz in molecular gases) and moving $\left(10^{3} \mathrm{~Hz}\right.$ to $\left.10^{5} \mathrm{~Hz}\right)$ striations [Garscadden (1978)].

Moving striations, also called ionization waves, are commonly the most readily identified and are the waves that play the role of coupled, nonlinear oscillators in this study. They are a ubiquitous phenomenon, even occurring in miniature in the tiny pixels of plasma television screens [Boeuf (2003)]. In many plasma applications, striations are a nuisance and this is one reason why much research has been done in this field [Rayment (1974)].

Typical ionization waves would occur in a discharge tube filled to around $200 \mathrm{~Pa}$ with monatomic gas, if it were operated between a few milliamperes and the Pupp limit [Pupp (1932)], the critical current above which the plasma is striation free. The Pupp limit is typically around $1 \mathrm{~A}$ or more, depending on tube geometry. For these conditions, the positive column glows with an apparent 
uniform luminosity. This outwardly calm behavior disguises ionization waves traveling too fast for the time resolution of the human eye [Ingold (1978)]. The observational range of existence of ionization waves is quite broad. Waves have been shown to exist in a pressure range $10^{-1}$ to $10^{+4} \mathrm{~Pa}\left(10^{-3}\right.$ to $10^{+2}$ Torr, the pressure unit commonly used in this field $)$ and within a range of discharge currents from $10^{-4}$ A to $10 \mathrm{~A}$ [Landa et al. (1980)].

The nature of these waves has been reviewed several times [Pekárek (1968), Oleson and Cooper (1968), Landa et al. (1980), and Kolobov (2006)] and and has been described in books [Franklin (1976) and Hirsch and Oskam (1978)]. The dispersion relation [see, for example, Pekárek (1968) or Oleson and Cooper (1968)] for ionization waves has the form $\omega=b+a / k$ where $a$ and $b$ represent constants that can be empirically matched to data from a specific discharge tube and $k$ is the wavenumber. Ionization wave propagation is peculiar because the group velocity is typically directed oppositely to the phase velocity. Waves with this feature are called backward waves and the waves in this study provide good examples. Phase velocities can range from $10^{3}$ to $10^{6} \mathrm{~cm} / \mathrm{s}$, and group velocities are comparable in magnitude but generally larger for low current waves [ Landa et al. (1980) and Kolobov (2006)].

Ionization waves can be categorized into four basic varieties, labeled $p, s^{\prime}, s$, and $r$. Each wave category is distinguished by a characteristic value for the potential drop over the length of a striation, known as the Novák constant $E \lambda$, where $E$ is the axial DC electric field and $\lambda$ is the preferred wavelength [Novák (1960)]. These values are tabulated by label and propagation in Table 1.1. The justification for using four categories can be seen in Figure 1.2 in which a neon discharge with a specific pressure was operated at a variety of discharge currents. At low currents, the Novák constant is independent of current whereas at high currents, $E \lambda$ is current dependent.

All four types are apparent when current and pressure are used to form a two-dimensional parameter space, as shown in Figure 1.3. At very low pressures, below about 1 Torr (or 2.5 Torr at currents $<1 \mathrm{~mA}$ ), the faster $r$ and $s$ waves dominate. At higher pressures, the slower $p$ and $s^{\prime}$ waves dominate. Between, there is a crescent-shaped region where no waves occur.

Convenient in characterizing the luminosity's spatial structure along the discharge tube is the use of reduced parameters [Pfau et al. (1969), Landa et al. (1980)]. Reduced pressure ( $p R$ ) and reduced current $(I / R)$ are obtained by combining each parameter with the radius $(R)$ of the discharge tube. This allows data from tubes of different radii to be plotted simultaneously. When this 
is done, it is clear from the overlap of data from different tube radii that the reduced parameters have the proper normalization.

One example of this is seen in Figure 1.4, in which the wave existence plot for neon is plotted on the reduced current-reduced pressure plane for differing tube radii ranging from $R=0.1 \mathrm{~cm}$ to $R=7.0 \mathrm{~cm}$. The data from the different tubes clearly follow the same pattern, and the crescentshaped wave-free region is shared by several data sets.

Convenient in characterizing the luminosity's temporal modulation by ionization waves is the reduced frequency $(R f)$, plotted against reduced current in Figure 1.5. The plot was made for a discharge with a pressure of $p R=1$ Torr $\mathrm{cm}$, similar to the 1.5 Torr $\mathrm{cm}$ tube used in this thesis. As in Figure 1.3, the ionization waves group neatly by type, but notice the periodic stair-step appearance in the $p$ waves. This stair-step feature indicates that $p$ waves manifest themselves in a discharge tube as a family of normal modes.

For the conditions of the neon discharge tube described in this thesis, $P=200 \mathrm{~Pa}$ (1.5 Torr) and discharge current from 3 to $15 \mathrm{~mA}$, it is clear from Figure 1.3 that the waves described in this work are $p$ waves. Since the radius of the tube is $1.0 \mathrm{~cm}$, and mode frequency ranges from 0.5 to $2.5 \mathrm{kHz}$, the reduced parameters of this tube are $p R=1.5$ Torr $\mathrm{cm}, 3 \mathrm{~mA} / \mathrm{cm}<I / R<15 \mathrm{~mA} / \mathrm{cm}$, and $0.5 \mathrm{~cm} \mathrm{kHz}<R f<2.5 \mathrm{~cm} \mathrm{kHz}$. With these ranges in mind, Figure 1.5 provides ample evidence to confirm the identity of $p$ waves in this study, even though the tube in Figure 1.5 and the tube used in this thesis have slightly different reduced parameters.

A straightforward physical picture can be used to explain the formation of ionization waves along the positive column. Within the axial electric field there, electrons gain energy until they achieve an amount necessary to ionize the neutral gas. At that point, ionization occurs, leaving low energy electrons to repeat the process [Landa et al. (1980)].

This simple picture has been supported with experimental studies of the electron energy distribution [Rayment (1974)]. While populations of electrons might be expected to get out of step with one another as they progress through the tube, they actually undergo "electron EDF (energy distribution function) bunching." Different wave types occur because of different resonances with the electron EDF. The $p$ and $s^{\prime}$ waves are slow waves and are metastable-guided, while the $r$ and $s$ are relatively faster and are ion-guided waves [Pekárek et al. (1970), Franklin (1976)]. When the pressure exceeds the Pupp limit, the electron EDF resonance is smeared and the waves are absent 
[Kolobov (2006)].

Theoretical models of the positive column assume cold ions and neutrals, and describe each species using fluid equations. Electrons are usually analyzed using kinetic analysis of the EDF. The characteristic energy scale is represented by the excitation threshold of atoms $\left(\varepsilon_{1}\right)$. The characteristic length is represented by $\lambda_{\varepsilon}=\varepsilon_{1} /(e E)$, which is the length necessary for an electron to achieve the excitation energy. A source term for ionization (and loss to the walls) must be included, and transport is considered to keep track of metastables in multiple-step ionizations [Kolobov (2006)].

When simulating ionization waves, partial differential equations modeling the plasma are combined with ordinary differential equations modeling the external circuit. Successful one- [Greiner et al. (1995), Jonas et al. (2000)] and two-dimensional [Arslanbekov and Kolobov (2003), Arslanbekov and Kolobov (2005)] simulations of striations have successfully reproduced experimental data.

\section{B. Nonlinear dynamics}

Nonlinear dynamics got its start in physics with the three-body problem of celestial mechanics, which proved unsolvable using the methods of Newton. Poincaré made notable advances in the late nineteenth century by taking a more geometric approach. He, it can be argued, was the first to notice the possibility of chaos, or aperiodic long-term behavior in a deterministic system that exhibits sensitive dependence on initial conditions. With the development of the computer, numerical approaches allowed the study of problems too intractable to do any other way [Strogatz (1994)].

Lorenz modeled atmospheric convection with a three-dimensional nonlinear computer simulation and discovered complex dynamics that was both deterministic and sufficiently dependent on initial conditions, even with unimaginable precision, to prevent long term prediction of behavior [Lorenz (1963)]. In other words, he accidentally rediscovered chaos. This seminal paper launched a tremendously productive period in nonlinear dynamics beginning in the 1970s, leading to numerous advances [Strogatz (1994)]. These include the discovery of fractal dimension, correlation dimension, and universal rules about the onset of chaos. These topics are beyond the scope of this thesis but are described in the popular press (most famously Gleick (1987)) and in textbooks (for example, 


\section{CHAPTER I. INTRODUCTION}

Strogatz (1994)).

In the late 1980s, the study of chaos occupied innumerable researchers across many subfields. This research was quickly extended to gas discharges, with the first experimental observation of chaotic behavior in a pulsed plasma discharge with a hot electron emitting filament [Cheung and Wong (1987)]. The following week, it was reported in a DC helium discharge tube manufactured for teaching spectral lines [Braun et al. (1987)]. In 1988, Cheung et al. (1988) documented the transition to chaos through intermittency, which is a route to chaos different from the perioddoubling route of the two studies above. The following year, Ströhlein and Piel (1989) documented the quasiperiodic transition to chaos in a driven plasma.

During the 1990s, experimental studies continued to proliferate. Weixing et al. (1993) documented the quasiperiodic transition to chaos in an undriven plasma. This study was documented using the Lyapunov exponent [Strogatz (1994)], a quantitative measure of a system's sensitivity to initial conditions. A positive value marks the transition to chaos, and the behavior of the exponent differentiates between fixed point, periodic, quasiperiodic, and chaotic behavior. A study of the Lyapunov exponent was done in 1995 for the neon glow discharge tube used in this study [Brown (1995)]

Using the method of Ott et al. (1990), Weltmann et al. (1995b) demonstrated that small, timedependent variations in the modulation amplitude could be used in an active feedback technique to stabilize chaotic orbits in a driven glow discharge. This so-called control of chaos marks another step in the understanding of the nonlinear behavior of glow discharge plasmas.

Despite its maturity as an experimental device, the discharge tube still contributes new science in the field of nonlinear dynamics. Dynamical classes can be studied, and behavior can be periodic, quasi-periodic, intermittent, or turbulent, depending on conditions [Kolobov (2006)]. The glow discharge is inherently nonlinear, as it is characterized by negative differential resistivity $(d V / d I<$ 0 ), and thus supports multiple modes that can influence each other if sufficiently large in amplitude and close in frequency. Introducing driving forces further enriches the dynamics. As an excellent example of a relaxation oscillator, both complete and incomplete (periodic pulling) entrainment by a periodically modulated driving force are readily demonstrated as experimental parameters are varied. Key concepts for interpreting the results in this thesis are spatial [Block (2001)], temporal [Koepke and Hartley (1991)], and spatiotemporal [Koepke et al. (2001)] entrainment, whether the 
entrainment is complete or incomplete. (These italicized terms and those that follow in the next section will be defined in Chapter II).

\section{Overview of thesis}

\section{Presented work}

This thesis summarizes an experiment in which a neon glow discharge tube operating in one of its normal modes is subjected to a driving force that is applied by chopped, neon-resonant, narrowband laser light. The chopping frequency is selected to approximately match an adjacent mode frequency (both the next-higher and next-lower mode frequencies were studied). The conclusion is that, under certain conditions, the neon glow discharge exhibits a behavior termed dynamics modulation, first seen by Koepke, Weltmann, and Selcher [Koepke et al. (1995)]. In dynamics modulation, two modes in a driven neon glow discharge alternate as the dominant mode as their response to the driving force alternates between spatiotemporal and temporal periodic pulling.

For two pairs of mode frequencies involved in the transition, three complete data series are experimentally observed. Ionization wave modes are examined using time series recorded using a photodiode with a narrow band filter that selectively passes the primary neon spectral line at 640 $\mathrm{nm}$. The system was periodically driven using a narrow-band ring dye laser tuned to a wavelength near the metastable neon transition at $588.35 \mathrm{~nm}$. The amplitude of the driving force was decreased (increased) by tuning the laser away from (nearer to) the center of the neon line, while the driving frequency was controlled by an acousto-optic modulator chopping the laser beam at the desired frequency.

Arnol'd tongue boundaries identifying the edges of frequency (or temporal) entrainment regions in the driving force's amplitude-frequency parameter space were established for four different discharge currents. The (upward) dynamics modulation behavior seen by Koepke, Weltmann, and Selcher was reproduced and additional data was acquired for two additional representative cases of downward modulations, previously undocumented. The upward modulations are used to verify the mechanism, while the downward modulations exhibit qualitatively different behavior.

Koepke, Weltmann, and Selcher proposed a mechanism by which dynamics modulation occurs [Weltmann et al. (2000)]. The modulation of the dynamics occurs as the entrainment (complete 


\section{CHAPTER I. INTRODUCTION}

or incomplete) alternates between temporal and spatiotemporal. For a pair of mode frequencies involved in the transition, two limited but representative cases were previously experimentally observed but the more exhaustive scrutiny contained in this thesis is used to establish the mechanism on much firmer footing.

An extension of the analytical treatment of periodic pulling of Lashinsky [Lashinsky (1968), Klinger et al. (1995)] is presented for the first time. This allows $M$, the normalized driving amplitude central to the proposed mechanism, to be accurately and directly calculated for spatiotemporal periodic pulling for the first time. In addition, the extension of the analytical treatment has implications for mode transitions in undriven discharge tubes, which will also be discussed.

In addition to the experiment, a numerical model is presented. Two square-wave driven, coupled van der Pol equations are chosen to model the behavior. The resulting time series was calculated with a Runge-Kutta routine whose parameters could be adjusted as the simulation proceeded to accurately reproduce the experiment. A comparison between the numerical study and the experimental results is presented.

\section{Organization of text}

In chapter II, the background and terminology necessary to understand this thesis are presented. This begins with background about the van der Pol equation, with emphasis on its use as a model for the nonlinear behavior of glow discharges (and other systems that support spontaneous oscillations). The analytical treatment of Lashinsky [Lashinsky (1968)] is presented and then extended to the case needed here, with driving frequency not approximately equal to the dominant mode frequency. How this analytical treatment is used to quantify the strength of the spatiotemporal and temporal periodic pulling is presented. Previous nonlinear dynamics work using a glow discharge tube is summarized in order to provide the experimental context for this study. Finally, dynamics modulation is defined and the motivating observation [Koepke et al. (1995)] and proposed mechanism [Weltmann et al. (2000)] are presented.

In chapter III, the experimental approach is described. This includes the details of the neon glow discharge tube, its external circuit, and how they are employed. The narrow-band laser is described, along with the acousto-optic modulator used to select the driving frequency and the method for calibrating laser wavelength using an iodine reference cell. The photodiode used for 
optical detection of the neon time series and the data acquisition method are also described. The method for determining the boundaries of the Arnol'd tongues is described. Finally, the diagnostic methods used to parameterize the nonlinear behavior of the plasma is presented, as well as how these methods are used to recognize signatures of distinctly different nonlinear behaviors.

Chapter IV contains the results of the experimental study presented in this thesis. This begins with the normal modes of the undriven discharge tube as each one first spontaneously arises and subsequently dominates as the discharge current is adjusted from one end of its range to the other end. Next, the Arnol'd tongue measurements of the driven discharge tube are presented, and the resulting boundaries are plotted as a function of laser wavelength for four different discharge currents. Regions where dynamics modulation was observed are documented, and dynamics modulation is demonstrated for upward modulation from each of two discharge currents, optimized to favor different, adjacent modes and thus best illustrate the dynamics modulation. The technique of quantifying periodic pulling strength, introduced in Chapter II, is extended to the more complex dynamics modulation case. Next, the results of this technique are presented as a function of driving frequency for the three experimental cases. Small segments of the time series are presented to emphasize the phase relationship between driver and oscillator during the two parts of a dynamics modulation cycle. Finally, two examples of downward dynamics modulation are presented.

In chapter $\mathrm{V}$, the coupled van der Pol equation model is presented. Starting with a successful recreation of upward dynamics modulation, a systematic study of its behavior is presented over a wide range of parameters. Results from the model as a function of driving frequency are compared to the experimental data. Finally, the model is used to attempt to reproduce the downward dynamics modulation seen in the experimental data.

A discussion of the experimental and modeling results appears in chapter VI, beginning with an evaluation of the proposed mechanism. The mechanism is evaluated in an item-by-item manner using experimental data, resulting in a quantitative and definitive verification of the mechanism. The model is then compared to the experimental results, and the outcome of the modeling process is evaluated. Last, the results of this study are placed in a broader context. Implications of the extended analytical model on mode changes in undriven neon glow discharges are discussed. Conclusions are presented in chapter VII. A derivation of the Lashinsky phase evolution formula used in chapter II and key MATLAB computer code used in this thesis are presented in appendices. 


\section{Chapter II}

\section{Background}

In this chapter, the van der Pol equation is introduced, and its mathematical properties are explored. As it is presented, relevant terms are defined and examples are given. Brief summaries of research important to establishing those ideas as experimental realities are presented. In the second section, experimental results establishing the usefulness of the van der Pol model in an electronic oscillator and a neon glow discharge are presented. The remaining nonlinear physics experiments relevant to this thesis are presented in the third section. These include the work of Koepke, Weltmann, and Selcher [Weltmann et al. (2000)], in which the mechanism for dynamics modulation was proposed.

\section{A. Characteristics of nonlinear oscillators}

\section{The van der Pol equation}

In his 1934 review paper titled The Nonlinear Theory of Electric Oscillators [van der Pol (1934)], Dutch physicist Balthasar van der Pol (1889-1959) presented a summary of his work with variations of the nonlinear equation that now bears his name. The van der Pol equation is the analytical starting point for studies of nonlinear behavior in systems such as the one in this thesis, and has been successfully used to model a wide variety of plasma systems [see Koepke et al. (1996) and references therein]. It is a nonlinear differential equation, here expressed with a driving force on the right hand side:

$$
\frac{d^{2} x}{d t^{2}}-\epsilon\left(1-\beta x^{2}\right) \omega_{0} \frac{d x}{d t}+\omega_{0}^{2} x=\omega_{0}^{2} E \cos \left(\omega_{i} t\right)
$$


In this equation, $x$ is the oscillating quantity, $E$ is the absolute amplitude of the driving force, $\omega_{0}$ is the normal mode frequency, $\omega_{i}$ is the driving frequency (using the convention of Lashinsky (1968), the subscript $i$ labels the "injected" frequency), and $t$ is time. The value of $E$ is assumed positive.

Here $\epsilon$ can be described as a parameter that determines the degree of nonlinearity. For $\epsilon<1$, the equation is weakly nonlinear, and for $\epsilon>1$, it is strongly nonlinear (if $\beta \neq 0$, of course). For $\epsilon \gg 1$, a relaxation oscillation is modeled [van der Pol (1934) and Minorsky (1962)]. The $\beta$ parameter adjusts the degree of nonlinear saturation [Klinger et al. (1995)]. The values of $\epsilon$ and $\beta$ are assumed to be positive.

Simply put, the van der Pol equation represents a self-oscillator. Neglecting the driving term for a moment, for $x^{2}<1 / \beta$, the damping is negative, causing the oscillator's amplitude to increase. However, for $x^{2}>1 / \beta$, the oscillator will have positive damping, which will tend to reduce the oscillation's amplitude [Baierlein (1983)]. The amplitude of the oscillator therefore will approach a steady state value from either larger or smaller values, tending to a stable limit cycle near the boundary in phase space where the damping changes sign [Strogatz (1994)].

Further intuition can be developed about the $\epsilon$ parameter. As $\epsilon$ becomes negligible, the effect of $\beta$ saturating the wave diminishes, damping diminishes, and a time-constant single frequency results. In the case of slightly larger $\epsilon$, specifically for $\epsilon \ll 1$, the expected undriven behavior is similar to that of a weakly dissipative harmonic oscillation. In this case, the wave would differ only very slightly from the familiar sine wave form. As $\epsilon$ becomes more significant, the shape of the wave can be expected to depart more and more significantly from a single-frequency sine wave.

In this thesis, the analysis of the van der Pol equation of Lashinsky (1968) and Adler (1946) is followed. Their work was summarized and extended in papers by Koepke and Hartley (1991) and Klinger et al. (1995), which are followed here for purposes of providing conveniently accessible further background. At the end of the section, the results are generalized for the case $\omega_{i} \nsim \omega_{0}$.

We start by assuming a solution to Eq. (2.1) of the form

$$
x(t)=a(t) \sin \left[\omega_{i} t-\phi(t)\right]
$$

which explicitly suggests that both the amplitude $(a)$ and phase $(\phi)$ are time dependent. Lashinsky 
used an analysis based on the variations of parameters and the method of averaging [Bogoliubov and Mitropolsky (1961)] to derive expressions for $a(t)$ and $\phi(t)$. It is necessary for this analysis to assume $\epsilon \ll 1$ and that $a(t)$ and $\phi(t)$ vary slowly so that second order time derivatives can be neglected.

If we further assume that $\omega_{i} \sim \omega_{0}$, so that $\left(\omega_{i}^{2}-\omega_{0}^{2}\right) / 2 \omega_{i} \approx \omega_{i}-\omega_{0}$, we can express the time derivative of $\phi$ as:

$$
\frac{d \phi}{d t}=\left(\omega_{i}-\omega_{0}\right)-\frac{E \omega_{0}}{2 a} \sin \phi \quad\left(\text { valid only if } \omega_{i} \sim \omega_{0}\right)
$$

The derivation of this equation is presented in Appendix A.

\section{Entrainment and the Arnol'd tongue}

If the driving frequency is sufficiently close to the natural frequency, for a given nonzero driving amplitude, the oscillator can become entrained to the driver. Entrainment means that the oscillation occurs at the frequency of the driving force, rather than at the natural frequency it would tend to in the absence of the driving force. Some authors, for example Adler (1946), refer to this phenomenon as frequency locking.

Since the phase of the driving frequency is assumed not to be time-dependent, entrainment requires that $d \phi / d t$ goes to zero. Examining Eq. (2.3), it is clear that the driving frequency is sufficiently close to entrain the oscillator when

$$
\left|\omega_{i}-\omega_{0}\right|<\frac{E \omega_{0}}{2 a}
$$

Following the convention of previous studies, the time dependence of $a$ is neglected and $a$ is considered to be positive [Lashinsky (1968), Klinger et al. (1995), and Weltmann et al. (2000)]. This neglect of the time dependence will be justified by the data later. The use of the absolute value ensures that frequencies will remain positive without loss of generality. If we define the conventional beat frequency as $\Omega_{0}=\left|\omega_{i}-\omega_{0}\right|$, we can write this condition as

$$
\Omega_{0}<\frac{E \omega_{0}}{2 a}
$$


Following Lashinsky (1968), we define an entrainment parameter $\alpha$. If $\alpha$ is defined as

$$
\alpha \equiv \frac{E}{2 a} \frac{\omega_{0}}{\Omega_{0}}=\frac{E}{2 a}\left|\frac{1}{\left(\frac{\omega_{i}}{\omega_{0}}-1\right)}\right| \quad\left(\text { valid only if } \omega_{i} \sim \omega_{0}\right)
$$

then entrainment will occur for $\alpha \geq 1$. (Note that Klinger et al. (1995) have a typographical error in their definition of $\alpha$, reversing $\omega_{0}$ and $\omega_{i}$.)

Furthermore, using this definition of $\alpha$, Eq. (2.3) can be rewritten as

$$
\frac{d \phi}{d t}=\Omega_{0}[1-\alpha \sin \phi] \quad\left(\text { valid only if } \omega_{i} \sim \omega_{0}\right)
$$

Figure 2.1 (from Klinger et al. (1995)) shows $\alpha$ graphically on the driving frequency-driving amplitude plane. The figure results from plotting Eq. (2.6) with $2 a=15$. The dark triangular region is the area where $\alpha \geq 1$, and therefore the wave is entrained. This region is called the Arnol'd tongue. Also shown are contours of $\alpha$ at $0.1,0.5,0.8$, and 0.9 outside of the Arnol'd tongue. The nearer $\alpha$ is to one, without actually reaching entrainment, the stronger the resulting periodic pulling. This is demonstrated in the next section.

It is apparent from the definition of $\alpha$ that there are three important parameters that determine proximity to the Arnol'd tongue. These are $E$ (the absolute amplitude of the driving force), $a$ (the amplitude of the oscillation), and $\omega_{i} / \omega_{0}$, the ratio of the driving frequency to the natural frequency. Figure 2.1 oversimplifies the picture by plotting the contours of $\alpha$ with $a$ assumed constant. In real Arnol'd tongues, these contours can be curved [see Figure 8 in Klinger et al. (1995) for example] due to frequency- and/or driving-force-dependent response amplitudes. Koepke and Hartley (1991) use an added term in their van der Pol model which can skew the response curve to the lower or higher frequency side.

To clarify the factors that influence periodic pulling, Koepke et al. (1996) combine $E / 2 a$ into a new parameter $M$, which is referred to as the normalized driving amplitude. Again, within this factor, the time dependence of $a$ is no longer explicitly considered. Koepke, et al. define $M$ as "one-half the ratio of the [absolute] driving force amplitude to the undriven system-response amplitude." Because the undriven amplitude would be zero for the temporal part of the dynamics modulation studied here, $a$ will represent the maximum driven amplitude in each portion of the 
dynamics modulation cycle, producing the most conservative estimate of $\alpha$.

The $\alpha$ parameter can then be described by:

$$
\alpha=M\left|\frac{1}{\tilde{f}-1}\right| \quad\left(\text { valid only if } \omega_{i} \sim \omega_{0}\right)
$$

where $M=E / 2 a$, one-half the absolute driving force amplitude divided by the maximum system response amplitude, and $\tilde{f}=\omega_{i} / \omega_{0}$, the ratio of the driving frequency to the natural frequency.

\section{Periodic pulling}

Periodic pulling is an interaction in which a nonlinear system undergoes a periodically interrupted attempt to become entrained when the system is just outside the Arnol'd tongue. Beginning when the driving frequency and the (slightly mismatched) self-oscillator frequency are in phase, the oscillator frequency is "pulled" toward the driving frequency. As each beat period progresses, the pulling increases and eventually saturates with the two frequencies still mismatched and the relative phase increasing. When the relative phase difference is too large (i.e., $\left.180^{\circ}\right)$, the self oscillator's frequency is no longer pulled and returns to its starting frequency. This change in frequency is accompanied by a change in amplitude. This simultaneous frequency and amplitude modulation repeats during the next beat. The pulling effect is strongest near the entrainment boundary, and falls off quickly as one moves away from the boundary. If the entrainment boundary is crossed during the frequency modulation, the the system will become entrained to the driving force [Adler (1946), Lashinsky (1968), and Koepke and Hartley (1991)].

Figure 2.2 shows the time series behavior for three cases of driving frequency. Using the van der Pol equation (2.1) with $\epsilon=0.4, \beta=0.1, \omega_{0} / 2 \pi=160 \mathrm{~Hz}$ and $E=1.5, \omega_{i} / \omega_{0}$ was selected to demonstrate weak periodic pulling (top panel, $\omega_{i} / \omega_{0}=1.2157$ ), strong periodic pulling (center panel, $\omega_{i} / \omega_{0}=1.1195$ ), and entrainment (bottom panel, $\left.\omega_{i} / \omega_{0}=1.05\right)$. The strong periodic pulling case has greater amplitude modulation than the weak periodic pulling case, and a more nonlinear time evolution.

The power spectrum of the three time series from Figure 2.2 is shown in Figure 2.3. The

entrained case (right panel) has a single peak at the driving frequency (168 Hz), as expected. The other two panels show beat-related sideband behavior that reflects periodic pulling. As the pulling 
becomes more non-sinusoidal, additional sidebands are required to describe the beat envelope. These sidebands are the result of describing non-sinusoidal frequency modulation with a timeaveraged spectrum.

For the weak periodic pulling case, the highest peak occurs at $161.3 \mathrm{~Hz}$, just above the natural frequency, and the second highest occurs at $194.7 \mathrm{~Hz}$, the driving frequency. The time-averaged sidebands are asymmetric with greater power on the low frequency side, opposite to the driving frequency. This would be reversed if the driving frequency were below the natural frequency. The sideband separation is $33.4 \mathrm{~Hz}$, and $\Omega_{0}=34.7 \mathrm{~Hz}$. For the strong periodic pulling case, the highest peak occurs at $179.3 \mathrm{~Hz}$, the driving frequency, and the second highest occurs at $171.3 \mathrm{~Hz}$, about $7 \%$ above the natural frequency. Sideband separation is $8 \mathrm{~Hz}$, and $\Omega_{0}=19.3 \mathrm{~Hz}$.

To explain this, we need to look closer at the idea of beat frequency. When periodic pulling occurs, the conventional notion of beat frequency is no longer adequate. The pulled system frequency departs from its undriven value and spends part of each cycle closer to the driving frequency. Therefore, one would expect a reduction in time-averaged beat frequency. The decreasing sideband spacing with growing periodic pulling strength represents the modified beat frequency, $\Omega$.

Mathematically, this can be arrived at by integrating Eq. (2.7). This can be done analytically [Adler (1946) and Lashinsky (1968)], and the result is:

$$
\tan \frac{\phi(t)}{2}=\sqrt{1-\alpha^{2}} \tan \left(\frac{\Omega_{0} t}{2} \sqrt{1-\alpha^{2}}\right)+\alpha .
$$

The beat frequency $\Omega$ of $\phi(t)$ can be found from the period of $\tan (\phi(t) / 2)$, and is simply

$$
\Omega=\Omega_{0} \sqrt{1-\alpha^{2}} \quad\left(\text { valid only if } \omega_{i} \sim \omega_{0}\right)
$$

Armand (1969) showed that if the output of the driven oscillator, Eq. (2.2), is expressed in complex notation and expanded in a Fourier series, the separation between the sidebands is $\Omega$. Looking back at Figure 2.3, and using Eq. (2.10), this suggests that the weak pulling case (left) has $\alpha=0.27$ and the strong pulling case (center) has $\alpha=0.91$. This is consistent with the statement that $\alpha$ values closer to one exhibit stronger periodic pulling.

Figure 2.4 further supports this pattern. This figure, from Klinger et al. (1995), is a series of nu- 


\section{CHAPTER II. BACKGROUND}

merical predictions of sideband frequency (a) presented alongside the corresponding instantaneous value of relative phase between the driver and the response signal (b). The top panels, in which the entrainment parameter $\alpha$ is relatively far from one, resemble conventional amplitude modulation [Koepke and Hartley (1991)]. In conventional amplitude modulation, a pair of sidebands appear around the natural frequency at $\pm \Omega_{0}[$ Cuccia (1952)]. In the rest of the panels, note that phase development becomes more nonlinear as $\alpha$ approaches unity. Time-averaged sideband separation narrows as $\Omega$ departs from $\Omega_{0}$, the conventional beat frequency. The spectrum becomes more and more one-sided, with the extra sidebands on the side opposite the driver.

The relationship between beat frequency and $\alpha$ can also be seen in Figure 2.5. This figure comes from the experimental data of Koepke and Hartley (1991), which is further described in the next section. It depicts beat frequency while moving across an Arnol'd tongue at constant driving amplitude. The entrainment boundaries are clear from the points where the time-averaged beat frequency drops to zero when the Arnol'd tongue region's center is approached from either side. The conventional beat frequency, $\Omega_{0}$, is represented by the dashed line, which approaches the entrainment boundaries linearly on either side. The observed beat frequency departs significantly from the dashed line, especially near entrainment where $\alpha$ approaches one, but agrees well with the solid line, which is calculated based on an equation similar to Eq. (2.10).

Figure 2.2 shows the amplitude modulation occurring during the periodic pulling cycle, but simultaneous frequency modulation is less clear. In order to see the frequency modulation, it is useful to introduce the idea of instantaneous frequency [Koepke and Hartley (1991) and Koepke et al. (1996)]. Instantaneous frequency is used to temporally resolve the frequency modulation, and can be calculated from the time series by inverting the time between zero crossings spaced an oscillation period apart. This practical description is consistent with more formal definitions of instantaneous frequency, such as that in Mallat (1999), but easier to use in practice.

Figure 2.6 shows the instantaneous frequency plot for the three series depicted in Figure 2.2. The bottom panel, as expected for an entrained oscillator, has constant frequency over the entire interval. The top panel, representing the weak pulling case, clearly exhibits a periodic frequency oscillation that corresponds in time to the amplitude oscillation visible in Figure 2.2. This is also true of the center, strong pulling case. In this case, however, the frequency modulation has long periods in which it is relatively steady, followed by short periods of rapid change. 


\section{Modified equations for $\alpha$ and $M$}

The assumption $\omega_{0} \sim \omega_{i}$ was used to get the equations above. However, this condition will not be true for the case of dynamics modulation, in which the driving frequency is selected so that it is not near the natural mode of the system, but near a neighboring subdominant mode. Since $\omega_{i}$ will differ from $\omega_{0}$ by as much as $20 \%$, this requires that the equations presented above be generalized to the case $\omega_{0} \nsim \omega_{i}$

We start with a more general version of Lashinsky's phase equation. (See Appendix A.) If we omit the assumption that $\omega_{i} \sim \omega_{0}$, we end up with a new version of Equation 2.3:

$$
\frac{d \phi}{d t}=\left|\frac{\omega_{i}^{2}-\omega_{0}^{2}}{2 \omega_{i}}\right|-\frac{E}{2 a} \frac{\omega_{0}^{2}}{\omega_{i}} \sin \phi \quad \text { (general form) }
$$

Here again, the absolute value symbols have been added in anticipation of the upcoming inequalities that will allow the entrainment boundary to be defined. This prevents the need for negative frequencies without loss of generality.

Above, $\alpha$ came from rewriting the equation in the form:

$$
\frac{d \phi}{d t}=\Omega_{0}[1-\alpha \sin \phi] \quad\left(\text { valid only if } \omega_{i} \sim \omega_{0}\right)
$$

If we work toward that form, again using $\Omega_{0}=\left|\omega_{i}-\omega_{0}\right|$, we find:

$$
\begin{aligned}
\frac{d \phi}{d t} & =\frac{\left|\omega_{i}-\omega_{0}\right|\left(\omega_{i}+\omega_{0}\right)}{2 \omega_{i}}-\frac{E}{2 a} \frac{\omega_{0}^{2}}{\omega_{i}} \sin \phi \\
& =\Omega_{0}\left[\frac{\omega_{i}+\omega_{0}}{2 \omega_{i}}-\frac{E}{2 a} \frac{\omega_{0}^{2}}{\omega_{i}} \frac{1}{\left|\omega_{i}-\omega_{0}\right|} \sin \phi\right]
\end{aligned}
$$

We see that $d \phi / d t$ can be zero when a new entrainment condition occurs. The new entrainment condition is:

$$
\begin{aligned}
\frac{E}{2 a} \frac{\omega_{0}^{2}}{\omega_{i}} \frac{1}{\Omega_{0}} & \geq \frac{\omega_{i}+\omega_{0}}{2 \omega_{i}} \\
\frac{E}{a} \frac{\omega_{0}^{2}}{\Omega_{0}} & \geq \omega_{i}+\omega_{0} \\
\frac{E}{a} \frac{\omega_{0}^{2}}{\Omega_{0}} \frac{1}{\left(\omega_{i}+\omega_{0}\right)} & \geq 1 .
\end{aligned}
$$


We identify the more general $\alpha$ as

$$
\alpha \equiv \frac{E}{a} \frac{\omega_{0}^{2}}{\Omega_{0}} \frac{1}{\left(\omega_{i}+\omega_{0}\right)}=\frac{E}{a}\left|\frac{1}{\left(\frac{\omega_{i}^{2}}{\omega_{0}^{2}}-1\right)}\right| \quad \text { (general form) }
$$

so that this new $\alpha$ has the same behavior as the less general $\alpha$ of Eq. (2.6), that is, the boundary for entrainment occurs at $\alpha=1$.

If $\omega_{i} \sim \omega_{0}$, it is clear that the $\alpha$ of Eq. (2.16) reduces to the previously defined $\alpha$, as it should:

$$
\begin{aligned}
\alpha & \equiv \frac{E}{a} \frac{\omega_{0}^{2}}{\Omega_{0}} \frac{1}{\left(\omega_{i}+\omega_{0}\right)} \\
& \approx \frac{E}{a} \frac{\omega_{0}^{2}}{\Omega_{0}} \frac{1}{2 \omega_{0}} \\
& \approx \frac{E}{2 a} \frac{\omega_{0}}{\Omega_{0}}
\end{aligned}
$$

Using $M=E / 2 a$ and $\tilde{f}=\omega_{i} / \omega_{0}$, a replacement for Eq. (2.8) is simple:

$$
\alpha=2 M\left|\frac{1}{\tilde{f}^{2}-1}\right| \quad \text { (general form) }
$$

Now, if we turn our attention to the modified beat frequency $\Omega$, Eq. (2.10) will also need to be replaced. This can be done by factoring the more general $\alpha$ of Eq. (2.16) out of Eq. (2.14):

$$
\begin{aligned}
\frac{d \phi}{d t} & =\Omega_{0}\left[\frac{\left(\omega_{i}+\omega_{0}\right)}{2 \omega_{i}}-\left(\frac{E}{a} \frac{\omega_{0}^{2}}{\Omega_{0}} \frac{1}{\left(\omega_{i}+\omega_{0}\right)}\right) \frac{\left(\omega_{i}+\omega_{0}\right)}{2 \omega_{i}} \sin \phi\right] \\
& =\Omega_{0} \frac{\left(\omega_{i}+\omega_{0}\right)}{2 \omega_{i}}[1-\alpha \sin \phi] .
\end{aligned}
$$

The expression in the parentheses is the new $\alpha$. If we then define the reference beat frequency $\Omega^{\prime}$ as

$$
\Omega^{\prime} \equiv \Omega_{0} \frac{\left(\omega_{i}+\omega_{0}\right)}{2 \omega_{i}}=\frac{\left|\omega_{i}^{2}-\omega_{0}^{2}\right|}{2 \omega_{i}}
$$


we can write

$$
\frac{d \phi}{d t}=\Omega^{\prime}[1-\alpha \sin \phi] \quad(\text { general form })
$$

Since Eq. (2.21) has the same form exactly as Eq. (2.7), it will have the same integral and it is clear that the beat frequency is

$$
\Omega=\Omega^{\prime} \sqrt{1-\alpha^{2}} \quad \text { (general form) }
$$

which is identical to Eq. (2.10) with $\Omega^{\prime}$ substituted for $\Omega_{0}$.

As long as the other assumptions used to derive the expression for $d \phi / d t$ are reliable, this gives a way to calculate $\alpha$, the generalized entrainment parameter, and $M$, the normalized driving amplitude, directly from the modified beat frequency $\Omega$ observed in the experiment. This general form of $\alpha$ and the other general equations of this section are used in the rest of this work.

\section{B. Experimental context}

In this section, previous research that provides context to the present study is presented.

\section{Nonlinear electronic oscillator}

Koepke and Hartley (1991) published a comprehensive study of periodic pulling in a nonlinear electronic oscillator built around a unijunction transistor (UJT). Although periodic pulling has been known since at least 1946 [Adler (1946)], this important phenomenon has received relatively little attention since, and was overshadowed by other work during the research boom on nonlinear science that began in the 1970s. Koepke and Hartley (1991) gave prominent placement to periodic pulling. In this paper, the authors document periodic pulling in a driven, nonlinear electronic oscillator and show that this behavior matches a theoretical treatment with the driven van der Pol equation.

The version of the van der Pol equation used in their model has an additional term when compared to Eq. (2.1). The extra term represents a nonlinear restoring force. The development of the theory follows that of Tsuru (1976), which is qualitatively similar to the development above, 
though quite different in notation. The effect of the additional term is to add an asymmetry in frequency to the response curves and therefore also to the Arnol'd tongues. This feature helped the authors fit their model to the data.

A figure from Koepke and Hartley (1991) reinforces the periodic pulling description above, and is reproduced in Figure 2.7. The figure shows a progression of driving frequencies from below the natural frequency and far from the Arnol'd tongue (a), through periodic pulling driven from the low frequency end (b through $\mathrm{f}$ ), through entrainment ( $\mathrm{g}$ and $\mathrm{h}$ ), then through periodic pulling from the high frequency end (i though $\mathrm{m}$ ) and finally from far above (n). The time series and the FFT magnitude are shown for each step in driving frequency. The qualitative behavior is clear. Asymmetric sidebands appear on the opposite side of the natural frequency from the driving frequency. The separation between the sidebands is reduced as entrainment is approached. The amplitude modulation gets more pronounced and more nonlinear as entrainment is approached. All of these behaviors are predicted by theory.

\section{Temporal vs. spatiotemporal periodic pulling}

The periodic pulling modeled above can be termed temporal periodic pulling. It results from interaction with a driving force that oscillates in time $\left(e^{-i \omega t}\right)$. If the nonlinear system can support multiple modes, it has been shown [Koepke et al. (1996)] that a significantly stronger form of periodic pulling, spatiotemporal periodic pulling, can occur. Koepke, et al. interpret this as being due to a second, subdominant mode pulling the dominant mode not only in time, but also along the length of the oscillation in space $\left(e^{i k z-i \omega t}\right)$ for a stronger effect.

The stronger effect is shown in Figure 2.8, from their study of ionization-wave-mode transitions in a gas discharge. In this figure, temporal pulling is shown along a curve similar to the right half of Figure 2.5. Close agreement is apparent between the theory, shown as a solid line, and the data, shown as individual points. (Different shapes represent beat frequencies calculated from different harmonics.)

The spatiotemporal case in their gas discharge study was not due to an external driving force, but rather due to a subdominant wave mode pulling the dominant wave mode while in the process of itself becoming dominant. Subplots (a1) and (a2) indicate the power spectra of two of these temporal pulling examples, while subplot (b) shows the power spectrum of the spatiotemporal 
case. The authors estimate that the spatiotemporal case has a comparable $\alpha$ to the temporal cases, but it does so from a greater frequency separation. Therefore, according to Eq. (2.8), it has a much larger $M$, and therefore is significantly stronger.

This result was the first identification of spatiotemporal periodic pulling. In later studies [Dinklage et al. (1998) and Koepke et al. (2001)], spatiotemporal dynamics in neon glow discharge tubes was further characterized. Dinklage et al. characterized $p$-wave mode transitions in time and space and related them to the instability theory of Eckhaus [Eckhaus (1965)]. Koepke, et al. characterized instantaneous frequency and instantaneous wavenumber in both space and time and identified signatures of the process.

\section{Optically driven waves}

Sheridan et al. (1993) used a chopped, narrow-band, neon-resonant laser to drive a neon-bulb relaxation oscillator. Dunham et al. (1991) were the first to study frequency entrainment in a laser-driven system. Their work followed that of Yan et al. (1990), who used the oscillator as an inexpensive but sensitive detector for spectral calibration. The work of Sheridan, et al. extended the work of Dunham, et al. by driving the oscillator near but outside the entrainment region. It showed unambiguous periodic pulling behavior.

The system was successfully modeled with a square-wave-driven van der Pol equation. Time series show relative phase between driver and response changing in a periodic fasion. Power spectra indicate the asymmetric character described above, with extra sidebands opposite the natural frequency from the driver. The work of Sheridan, et al. is directly relevant to the present study, which uses the same method of driving the oscillation.

Weltmann et al. (2000) used this same technique to drive a neon-glow discharge. They found the driving technique to work well and used it to confirm the spatiotemporal wave-wave interaction first documented by Koepke et al. (1996). They also presented characteristic power spectra and instantaneous frequency series that can be used to distinguish between temporal and spatiotemporal periodic pulling. (These signatures are discussed in Chapter III.)

In addition, they documented several other behaviors of the system. They showed that neonresonant, DC, laser light can cause mode transitions in the discharge as the position of the laser is moved along the plasma column. They showed that chopped, neon-resonant laser light can 
cause periodic pulling when the frequency is set just outside the entrainment boundary, and they showed that the entrainment boundaries themselves will appear different if approached from inside entrainment moving out, rather than outside moving in. Finally, and central to this work, they presented a published record of the phenomenon they named dynamics modulation, first seen by Koepke et al. (1995).

\section{Dynamics modulation}

While conducting investigations using chopped, neon-resonant laser light to systematically control spatiotemporal periodic pulling in a neon glow discharge, Koepke et al. (1995) noticed a peculiar phenomenon. They reported this discovery in Weltmann et al. (2000), along with a proposed mechanism. In this section, the phenomenon is first described, then the mechanism of Koepke, Weltmann, and Selcher is presented and then summarized as itemized elements to be verified.

While the discharge was optimized at one mode, they were able to entrain, using chopped, neon-resonant laser light, a subdominant mode at a frequency near but just below the next higher normal mode frequency and have that mode spatiotemporally pull on the dominant mode. As they increased the driving frequency toward the spontaneous frequency of that higher mode, the pulling became stronger. Eventually, it reached a strength capable of triggering an upward mode transition in the system, but this upward mode transition was short-lived. Despite the laser light's ability to force an upward mode transition, it was unable to entrain the now-dominant higher mode. Soon afterward, the lower mode regained its original dominant status. The process then repeated.

This is shown in Figure 2.9, from Weltmann, et al., which shows the instantaneous frequency signature of the phenomenon. This signature is a clear change in the median frequency about which the frequency oscillation occurs, repeating periodically. The lower mode, dominant throughout most of the time series, is at $1.5 \mathrm{kHz}$ and is referred to as $f 7$. The higher mode $(f 8)$ is at $1.75 \mathrm{kHz}$, and the driver in this case (which entrains the subdominant mode when the lower mode dominates) is at $1684 \mathrm{~Hz}$. Also shown in this figure is the characteristic "hairy" power spectrum. The term dynamics modulation refers to this incomplete transition between two ionization wave modes, which occurs, reverses, and repeats periodically.

The reason for the modulation was formulated as a result of the two representative cases that were observed. The first part of the explanation is that the amplitude of the higher mode increases 
immediately after the mode flips up. With this increase in amplitude, a decrease in $M$ results from Eq. (2.8). This reduction in the normalized driving strength brings $\alpha$ below one and therefore interrupts the entrainment of the higher mode. The higher mode reverts to its natural frequency, which is briefly temporally pulled by the nearby laser chopping frequency but is not very stable as a limit cycle. Since the lower mode can be expected to survive for times on the order of 10 ms [Koepke et al. (1996)], it does not decay immediately after the mode transition. It regains its original dominant status, causing a reversal of the change in $M$. Then the process repeats.

As the driving frequency gets even nearer to the higher mode from the low frequency side, the relative time spent in each phase of the modulation changes. In a second figure (not reproduced here), Weltmann, et al. show that for a driving frequency of $1688 \mathrm{~Hz}$, the dynamics modulation up to a higher mode occurs earlier and remains a little longer. They attribute the difference to the faster relative phase evolution of the driver and the dominant lower mode, leading to a faster mode transition up, and to the slower phase evolution between the driver and the natural frequency of the higher mode, leading to a longer stay in the higher mode before returning to the lower mode. These arguments are explored in Chapter VI, where the proposed mechanism is evaluated.

Figure 2.10 from Weltmann et al. (2000) summarizes the dynamics modulation phenomenon. This diagram represents a schematic Arnol'd tongue situation, with the vertical axis representing the parameter $M$. The lower and upper modes are identified by their labels $f 7$ and $f 8$, respectively. The lines represent Arnol'd tongue boundaries for the two modes. All points on the figure can be imagined as resulting from the same absolute driving force, with the differences in their vertical position being due to differences in the amplitude of the oscillations involved. That is, $E$ is constant, but $a$ is changing in Eq. (2.8) so the position on the vertical axis (i.e., $M$ ) is changing. This figure is central to the proposed mechanism of dynamics modulation presented by Weltmann, et al.

The $\triangle$ represents a case of temporal periodic pulling near the dominant mode. Since the amplitude $(a)$ of the dominant mode would be large, $M$ is proportionally smaller. As the driving frequency is increased to near but below the subdominant mode, it can entrain that mode, but at a much lower amplitude than the dominant mode. The $\boldsymbol{\nabla}$ represents this location, which appears at a correspondingly higher $M$. In this location, strong spatiotemporal periodic pulling occurs, but the pulling is inadequate for triggering a mode transition upward.

The - and cases represent two cases of dynamics modulation. Both are further from the 
dominant mode, and so would have even smaller $a$ values due to the diminishing response of the $f 7$ mode at that distance. Therefore, they would have even larger $M$ positions than the $\mathbf{\nabla}$, and the appears slightly above the $\bullet$ since it is farther away. For these cases, the spatiotemporal periodic pulling is stronger than for the $\mathbf{\nabla}$, and is in fact strong enough to cause the higher mode to become dominant briefly. However, this change in dominance changes the amplitude $a$, which changes the effective amplitude of the driving force $M$ on Figure 2.10. The change in $M$ that occurs when the mode shifts to the higher, normally subdominant mode is indicated by the $\circ$ and the $\square$. These appear outside the entrainment boundary, leading to a return to dominance for the $f 7$ mode. The whole process then repeats. Within the dynamics modulation region, the amount of time spent at the higher mode increases as the driving frequency approaches the upper entrainment boundary. The two circles (• and o) correspond to the case shown in Figure 2.9, and the squares to a nearby case with a different driving frequency and modulation period.

As the driving frequency is further increased, the modulation ceases and the higher mode becomes permanently entrained by the driver. The represents this case. The subdominant mode becomes the dominant mode which, in this case, does become entrained, and no dynamics modulation occurs.

Weltmann, et al. provide additional detail in their explanation of the process. In addition to the description above, there are predictions about the timing of upward mode change and the downward mode return. Specifically, they propose that the upward mode transition occurs when the higher mode, and the laser light to which it is synchronized, is "sufficiently out of phase the the [lower] mode." They also attribute the cycle's end to the temporal pulling cycle of between the laser and the upper mode, stating that "the laser's temporal pulling of the [higher] mode is strong enough to interrupt the consummation of the upward mode transition before the [lower] mode decays completely" at which point the system returns to the lower frequency. Third, they propose that, during the time spent in the upper mode, the temporal periodic pulling process occurs in reverse from the normal case described in the first section of this chapter, namely, the cycle begins with the driver and the mode in-phase and evolves to the out-of-phase point.

This proposed mechanism for dynamics modulation has not been experimentally tested until now. An experiment was conducted in which the dynamics modulation of Koepke et al. (1995) was reproduced and studied in detail. This allowed a series of specific predictions to be tested as a 
verification test for the proposed mechanism. These predictions can be summarized as follows.

1. The system's $M$ during the upper temporal part of the dynamics modulation cycle is reduced compared to the $M$ during the lower spatiotemporal part of the cycle.

2. The system alternates between laser entrainment of the subdominant upper mode during the lower frequency part of the cycle and temporally pulling but not entraining the (now dominant) upper mode during the upper part of the cycle.

3. The occurrence of dynamics modulation, relative to the Arnol'd tongue, is represented accurately by their "dynamics phase diagram," shown in Figure 2.10.

4. The change in $M$ is consistent with a change in amplitude in the laser-light-driven upper mode.

5. The upward modulation occurs when the instantaneous frequency of the lower mode is pulled sufficiently close to the upper mode.

6. The upward modulation is related to the relative phase evolution between the lower mode and the laser-entrained upper mode, occurring when they are out of phase.

7. The downward, return modulation occurs when the chopped laser light and the temporally pulled upper mode are back in phase.

8. The temporal pulling of the upper mode during the higher frequency portion of the dynamics modulation is associated with a relative phase between the system and the driving force that evolves in reverse.

The first four items listed result from the dynamics modulation mechanism itself. The remaining items describe the timing and phase relationships that occur during dynamics modulation. All items are discussed in the chapters that follow, and the proposed mechanism is evaluated.

Additional novel cases of downward dynamics modulation, in which the laser chopping frequency is placed near the next lower mode, were observed for the first time during this study. These cases did not exhibit the same qualitative behavior as the upward cases described by Koepke, et al., and therefore are presented and discussed separately. 


\section{Chapter III}

\section{Experimental Approach}

In this chapter, the experimental apparatus is described in detail in the first section. Following that, the diagnostic methods used to perform the experiment are explained and the signatures of the pertinent dynamics are presented.

\section{A. Experimental arrangement}

\section{Neon glow discharge tube and circuit}

The centerpiece of this experimental study is a neon glow discharge tube. The discharge tube is powered by a Series ER 6 kV High Voltage Power Supply manufactured by Glassman High Voltage, Inc., arranged to have negative voltage output, and operated through a load resistance of $100 \mathrm{k} \Omega$. Although the exact load resistance is not crucial, such external circuit elements do have an effect on the Arnol'd tongue boundaries [Weltmann et al. (1995a)], and therefore the load resistance was selected to match that of Weltmann et al. (2000), whose observations we sought to reproduce as a starting point.

The discharge tube contained pure neon. The parameters of the discharge tube itself were taken from markings on the outside of the tube. The radius $(R)$ was $1.0 \mathrm{~cm}$, and the pressure $(P)$ was listed as 1.5 Torr (200 Pa) at room temperature. The length of the tube between anode and cathode was $70.0 \mathrm{~cm}$. The Glassman power supply was operated in current control. The current delivered by the power supply was measured by monitoring the "TB7" contact on the back of the power supply, which outputs a voltage proportional to the current delivered. The waves typically 
appeared beginning at $3 \mathrm{~mA}$ and were observed up to the top of the range $(18 \mathrm{~mA})$ selected to prevent high-voltage breakdown of the RG-58 cable used. There were nine normal modes of $p$-waves possible within this range, with frequencies between $640 \mathrm{~Hz}$ and $2 \mathrm{kHz}$. (These will be documented in Chapter IV.) Two internal discharge-encircling rings near the cathode end were not used in this application.

The complete discharge tube circuit, along with the chopped-light driving force and data acquisition systems, are schematically presented in Figure 3.1. Each component of the experimental arrangement is further described in the subsections that follow.

\section{Narrowband dye laser}

The optical forcing was accomplished using a Coherent 899 ring dye laser, operated with Rhodamine 6G fluorescent dye, pumped with an Innova 90 Plus Argon-Ion laser, and tuned to wavelengths near the metastable neon transition at $588.35 \mathrm{~nm}$ [Meggers and Humpreys (1934)]. (The precise value is $588.35252 \mathrm{~nm}$, according to Ralchenko et al. (2008).) This vacuum wavelength corresponds to $588.19 \mathrm{~nm}$ in air and represents the $2 s^{2} 2 p^{5}\left({ }^{2} P_{3 / 2}^{\circ}\right) 3 s$ to $2 s^{2} 2 p^{5}\left({ }^{2} P_{1 / 2}^{\circ}\right) 3 p$ transition [Wiese et al. (1966)]. In the older Paschen notation commonly used in discharge physics, this is represented as $1 s_{5}-2 p_{5}$ [Moore (1971)]. This neon transition is different than that used by Weltmann et al. (2000), but serves the same purpose, neon-resonant driving, in a similar way.

The laser was capable of delivering the necessary driving force when operated at a power between 100-150 mW (as measured just outside the laser cavity's output coupler), well below the maximum available power at this wavelength. Laser power was monitored and the position of the laser and optics relative to the discharge tube was not adjusted during the experiments in order to ensure a constant absolute driving amplitude.

A commercial wave meter (Bristol Instruments series 621) was used to guide the tuning of the laser to the approximate location of the resonant neon line. Fine calibration of the laser wavelength was accomplished using an iodine reference cell. As the wavelength of the laser was scanned, a diverted portion of the light beam passed through the iodine cell and the resonant absorption of the iodine gas was recorded using the output of a dedicated photodiode. Two iodine lines, identified as numbers $372\left(\right.$ at $16996.6245 \mathrm{~cm}^{-1}$ ) and $373\left(\right.$ at $16996.6242 \mathrm{~cm}^{-1}$ ) in the iodine atlas [Gerstenkorn and Luc (1977)] were used to establish an absolute wavelength scale in the 
neighborhood of the neon-resonant bandwidth.

Note that Keesee (2006) and Scime et al. (2007) recently brought attention to a previously documented [Gerstenkorn and Luc (1985)] systematic error in the iodine atlas that must be considered when the iodine cell is used for wavelength calibration in laser induced fluorescence (LIF) studies. As detailed in Gerstenkorn and Luc's Figure 9, this error would be expected to alter wavenumbers

near $17000 \mathrm{~cm}^{-1}$ by no more than $0.002 \mathrm{~cm}^{-1}$. An error of this size would be expected to cause a wavelength calibration error of $\approx 7 \times 10^{-5} \mathrm{~nm}$. While a miscalibration of this magnitude would introduce a detrimental error level into LIF velocity measurements, the wavelength calibration here is used only to provide a reproducible reference for driving-force amplitude. A systematic error, particularly one this small, does not affect this purpose and therefore was not eliminated.

Sample output from the iodine cell, fit to data from the iodine atlas, is shown in Figure 3.2. In this figure, the circles represent the output from the iodine cell. The dashed line is the fit based on the lines from the iodine atlas, and establishes the operation range of the calibration. This figure will also be discussed below in the diagnostic methods section.

\section{Controlling driving frequency}

The driving frequency was controlled with an Isomet $1205 \mathrm{C}-2$ acousto-optic modulator (AOM) driven by an Isomet 222A-1 AOM driver. This device effectively chopped the laser light between off $(0 \%)$ and on $(100 \%)$ states at a frequency selected using a manually adjusted Agilent 33220A function generator in square-wave mode.

The laser beam was reflected upward, passed vertically through the AOM, and then was directed toward the discharge tube so that the first-order AOM output line was directed at a point on the discharge tube $2.5 \mathrm{~cm}$ from the cathode. This point was selected because it has excellent coupling to the plasma. (See Figure 8 in Weltmann et al. (2000).) Physically, this target point appears to be the center of the "negative glow" feature of the discharge.

\section{Data acquisition}

A photodiode detector was constructed by placing an optical assembly in front of a simple negativelybiased photodiode circuit. This design of this detector is displayed in Figure 3.3. The detector was placed at a position $57.5 \mathrm{~cm}$ from the cathode and aimed at the center of the discharge. The 
photodiode detector was elevated so that its axis was at a height matching the axis of the tube. The distance between the detector and the tube was adjusted so that the center of the tube was one $50 \mathrm{~mm}$ focal length from the first lens in the detector $(25 \mathrm{~mm}$ diameter, Edmund Optics part number NT32-624). The light then passed through a $640.1 \mathrm{~nm}, 4 \mathrm{~nm}$ bandwidth filter before being focused by a second identical lens onto a photodiode.

The lenses and filter were properly oriented by an optical assembly (Figure 3.3a) made from common household PVC "Schedule 40" plumbing parts of appropriate diameter to hold the optics in position. Light entered an aperture created by a 1-inch-to- $\frac{3}{4}$-inch reducing adapter with unthreaded fittings, which held one lens and the filter. The second lens was held in place with

a $\frac{3}{4}$-inch unthreaded-to-threaded adapter. Finally, the photodiode was held by a $\frac{3}{4}$-inch threaded cap. Spacers were made from small sections of $\frac{3}{4}$-inch pipe cut square on the lathe, a longer section of pipe, and (as a pad between lens and filter) a 1-inch OD, $\frac{3}{4}$-inch ID O-ring. The threaded connection allowed the distance between the photodiode and the second focusing lens to be optimized for the best-quality signal. The pieces were held in place by friction, and shielded from outside light in an aluminum enclosure.

The photodiode circuit (Figure $3.3 \mathrm{~b}$ ) was powered by a $9 \mathrm{~V}$ battery using a 7805 voltage regulator, wired to produce a $-5 \mathrm{~V}$ output. Light incident on the photodiode generated a current which traveled to ground through a $220 \Omega$ resistor. The voltage across this resistor gave a signal which was passed to a Stanford Research Systems model SR560 preamplifier before being recorded in a LeCroy Waverunner 6100A digital oscilloscope. Typically, the gain on the amplifier was set at 200, and no filtering was used.

\section{B. Diagnostic methods}

\section{Time series analysis}

The primary data source for this study of dynamics modulation consists of time series recorded from the photodiode, which records the oscillations of the luminous ionized gas in the discharge tube. These time series were recorded so that they had excellent time resolution (typically 100 $\mathrm{kS} / \mathrm{s}$ for $1 \mathrm{~s}$ ) and multiple realizations were typically recorded for each driving frequency.

It is well known that a periodic time series can be decomposed using a Fourier transform 
into the frequency domain. Two methods of doing this were employed in this study. First, the digital oscilloscope used could directly record spectral information based on its internal fast Fourier transform (FFT) routine. This method was used for the mode frequency/discharge current map (Figure 4.1) and for purposes of identifying interesting regions during data collection (such as identifying Arnol'd tongue boundaries, described below.) The other method was to process the time series later, and used the standard FFT routine built into the MATLAB programming language to create auto-correlation power spectra.

In addition, a routine was created [Koepke and Hartley (1991), Weltmann et al. (2000)] to calculate the instantaneous frequency time series, in order to recognize signatures of temporal periodic pulling, spatiotemporal periodic pulling, and dynamics modulation. These signatures will be described below. These plots were also used to identify the precise moments when the dominant frequency was modulated upward or downward, so that the time spent in each part of the modulation could be known and phase evolution between two known frequencies during these times compared to the proposed mechanism of the Weltmann, et al. paper.

\section{Establishing the boundaries of the Arnol'd tongue}

Central to the mechanism proposed by Weltmann et al. (2000) is the occurrence of dynamics modulation relative to the Arnol'd tongue boundaries of the involved modes, shown in their dynamics modulation phase diagram (reproduced in Figure 2.10). This requires an experiment designed to test the mechanism, such as this one, to gather data that definitively establish the Arnol'd tongue boundaries. Although such a figure has not been created previous to this study, the process is straightforward.

One requirement is to have some method for measuring the absolute amplitude of the driving force. This method, described above, is to measure wavelength of the narrowband incident light. Due to the non-negligible temperature of the neon ions, the population of ions is spread around the center of the spectral line at $585.35 \mathrm{~nm}$. More of the particles will resonate to laser light tuned near the center of the line. As the laser is tuned away from the line, fewer metastable neon atoms will resonate.

This effect is clear in Figure 3.2. The outer triangles on the axis represent the approximate wavelength extremes at which the laser just begins to affect the discharge. The interaction between 
the laser and the discharge becomes more pronounced as the laser is tuned inward from the triangles on either outer edge. Between the inner pair of triangles, the laser is resonant enough to entrain any frequency despite the preferred natural modes of the plasma. The center of the resonant line, though not precisely measured here, is between the two inner triangles. Note that the actual line is slightly offset from the vacuum value of $585.35252 \mathrm{~nm}$ because the inside of the neon discharge tube is not a vacuum.

The two five-pointed stars have been placed at representative wavelengths where dynamics modulation has been observed. (A similar dynamics modulation regime would be expected on the opposite side of the peak resonance.) From Figure 3.2, it is clear that a progression of absolute driving amplitude can be experimentally produced by controlling the wavelength of the narrow-band laser, and that this will serve as the $y$-axis on an Arnol'd tongue plot.

The second requirement for producing an Arnol'd tongue map is to have a way of establishing the boundaries of the Arnol'd tongue itself. This can be done by examining the real-time FFT output of the photodiode cell as the driving frequency is controlled at a given laser wavelength. Once the wavelength of the laser has been established, the driving frequency in the vicinity of the boundary has a distinct effect, which is shown in the sample data of Figure 3.4.

During this series, the discharge current was set at $9.51 \mathrm{~mA}$, which places the discharge so that its mode frequency is around $1440 \mathrm{~Hz}$ (see Chapter IV). The driving frequency is shown on the left side of each of the power spectra, and it is driving the mode from below. Consequently, extra sidebands indicating periodic pulling appear on the right. As one views the panels from top to bottom, the driving frequency increases, and the periodic pulling gets stronger as indicated by the reduced separation between the sidebands. Finally, in the bottom panel here, the oscillation is entrained to the driving frequency.

Although the sideband separation can get smaller and smaller and may go below the spectral resolution of the data, the effect of becoming entrained is unmistakable on the oscilloscope, particularly at lower amplitude. The spectral feature visually changes from asymmetric to symmetric and from subtly variable with each update to smooth and still. At this point, the driving frequency was recorded to define the entrainment boundary.

All available entrainment boundaries for a given driving amplitude were recorded at each step. Then, the first recorded entrainment boundary was checked again to ensure that it remained the 
same. If it had changed, a mode hop in the laser during data collection would be indicated and the data point would have to be repeated.

Because an already-entrained oscillation will tend to stay entrained, entrainment boundaries will appear wider if they are located by going from inside entrainment out [Weltmann et al. (2000)]. For this study, entrainment boundaries were always located by starting outside entrainment and moving toward entrainment.

\section{Signatures of distinct behaviors}

\section{Temporal periodic pulling and entrainment}

Periodic pulling, as described in Chapter II, consists of a time signal that is undergoing a periodically repeating oscillation in its frequency due to the presence of a nearby driving frequency. A simultaneous amplitude modulation is also present. In this subsection, we review the signatures of periodic pulling and entrainment.

The power spectrum signature is the clearest sign available during real-time data collection. An example of this is shown in Figure 3.5. Here, a corresponding view of the time series is also shown, with the time axis enlarged to show the individual oscillations. A quick glance at the time series suggests a very uniform oscillation, with no hint of periodic pulling aside from some amplitude modulation. There is certainly no obvious feature to suggest that the driving frequency (here at $1522 \mathrm{~Hz})$ is above the undriven oscillation frequency $(1466 \mathrm{~Hz})$ rather than below. So the power spectrum is the first diagnostic needed to see temporal periodic pulling.

In Figure 3.6, the instantaneous frequency signature of temporal periodic pulling is shown (bottom). This figure displays the same data as Figure 3.5, so the driving frequency is above the dominant mode frequency. The instantaneous frequency has a clear beat, with the beat frequency being the $\Omega$ factor discussed in Chapter II. Here, that beat frequency is about $42 \mathrm{~Hz}$, since here there are about 10.5 beats in 0.25 seconds. Using Eq. (2.10), it is simple to calculate $\alpha$ for this case: $\Omega_{0}=|1522-1466|=56 \mathrm{~Hz}$ and $\Omega=42 \mathrm{~Hz}$, so $\alpha \approx 0.66$. Using the frequencies and Eq. (2.8), $M \approx 0.025$, comparable to the values reported by Koepke et al. (1996) for temporal periodic pulling.

In the top half of Figure 3.6, the corresponding time series is shown. While the oscillations occur 
so fast that they darken the entire region, it is clear that their overall amplitude is modulated along with the frequency modulation. This amplitude modulation was also seen in Figure 2.7, but here, with the use of the instantaneous frequency series, it is seen to be exactly out of phase with the frequency modulation, with frequency peaks corresponding to amplitude minima.

The degree of amplitude modulation apparent for the relatively strong $(\alpha=0.66)$ temporal pulling in Figure 3.6 is modest, with variation comprising $5 \%$ of the maximum amplitude. This shows that the neglect of the time variation of $a$ in Chapter II during the derivation of $\alpha$ and $M$ is an acceptable assumption.

The shape of the instantaneous frequency series during a beat in Figure 3.6 is an arc in a concavedownward orientation. This shape reflects the behavior of the frequency modulation. The driving frequency is pulling the oscillator frequency up. This shape shows that more time in the cycle is being spent at high frequencies, with the return to low frequencies during each cycle representing a quick reset.

In Figure 3.7, this case (bottom) is shown alongside another case (top), where the driving frequency is below (at $1430 \mathrm{~Hz}$ ) for the same spontaneous mode frequency. Here, more time is spent at lower frequencies, so the instantaneous frequency beat period arc appears concave up. Also clear from this figure is that the sidebands appear on the opposite side of the oscillation from the driving frequency.

While the beat frequency $\Omega$ can be transparently obtained from the instantaneous frequency time series, it can also be obtained from the separation of the sidebands in the power spectrum. Using this method on the top (bottom) half of Figure 3.7, one obtains a value of $35.8 \mathrm{~Hz}(41.6 \mathrm{~Hz})$ for $\Omega$.

\section{Spatiotemporal periodic pulling}

Despite some similarities between the signatures of temporal and spatiotemporal periodic pulling, it is misleading to think of the two as different extremes of the same single phenomenon. The main difference between the two kinds of pulling is their strength. In order to have a strong effect, temporal periodic pulling must occur at frequencies near entrainment of the mode frequency. Spatiotemporal periodic pulling can have these same strong effects, but can do so from frequencies relatively far away from the main mode frequency [Koepke et al. (1996)]. The reason for this, to 
repeat a point from Chapter II, goes to the heart of the difference between the two. Spatiotemporal periodic pulling occurs when one wave mode drives another along its length in space as well as in time; temporal periodic pulling occurs in time only.

Even though this fundamental difference exists, some comparisons can be drawn between the signatures of the two phenomena. A case of spatiotemporal periodic pulling is shown in Figure 3.8. Here, the discharge current was set at $14.0 \mathrm{~mA}$, which makes the undriven mode frequency $\sim 1714$ $\mathrm{Hz}$ (see Figure 4.3). The driving frequency in this case was $1455 \mathrm{~Hz}$, which is near the mode below. (This mode would be dominant at lower discharge currents.)

In the top half of the figure, the power spectrum is shown. Because of the asymmetric sidebands on the higher frequency side, it clearly shows periodic pulling with the driving frequency below the dominant frequency of oscillation. But when the instantaneous frequency is examined, in this case, it is clear that the periodic pulling is much stronger than it was in the case of Figures 3.5 to 3.7. Note that the concave-up appearance is still present, indicating more time spent at lower frequencies in the oscillation, as expected for downward pulling. But the range of oscillation is much wider. In this spatiotemporal case, the instantaneous frequency oscillates through a range of about $750 \mathrm{~Hz}$. Compare this to the temporal case shown in Figure 3.6, where the oscillation range is around 100 $\mathrm{Hz}$.

Meanwhile, unlike in the temporal case, an estimate of $\alpha$ cannot be found directly from Eq. (2.10). Here, the separation between the sidebands $(\Omega)$ is $282 \mathrm{~Hz}$, while the conventional beat frequency $\left(\Omega_{0}\right)$ is $259 \mathrm{~Hz}$. With $\Omega>\Omega_{0}$, Eq. (2.10), which assumes the driving frequency is near the spontaneous mode frequency, fails.

The alternate form of $\alpha$ in Eq. (2.22) can be used to get a value of $\alpha$. Estimating the spontaneous mode frequency as $1714 \mathrm{~Hz}$ (see Figure 4.3) for $14.00 \mathrm{~mA}, \Omega^{\prime}$ (from Eq. (2.20)) is 282.05. Since $\Omega<\Omega^{\prime}$, it is possible to get a value for $\alpha$, giving $\alpha=0.02$.

A close up of the time series (top) and the instantaneous frequency series (bottom) is shown in Figure 3.9. Again it is clear that the amplitude modulation tracks with the frequency modulation, with frequency peaks occurring simultaneously with amplitude minima. The frequency oscillation is also visible in the time series itself, lending a visual connection to the instantaneous frequency plot below. 


\section{Dynamics modulation}

The two signatures of dynamics modulation are shown in Figure 3.10. For these data, the discharge current was set at $9.10 \mathrm{~mA}$, so that the undriven mode would occur at about $1450 \mathrm{~Hz}$. The driving frequency was set to $1704 \mathrm{~Hz}$. The top half of the figure shows the distinct "hairy" spectrum which results from the modulation time series. The two largest features are centered around the driving frequency and the undriven mode frequency. This new example of dynamics modulation compares well with the earlier published result of Weltmann et al. (2000), seen in Figure 2.9, differing only in frequency of modulation.

The bottom half of the figure shows the instantaneous frequency time series. It is clearly switching between frequency oscillations centered around the lower frequency favored by the discharge in its undriven state, and oscillations around the higher mode, close to the driving frequency. This clear mode switching, combined with the characteristic look of the spectrum, signals dynamics modulation.

The behavior of the instantaneous frequency before and after transitions is typical of more conventional mode transitions. The amplitude of the frequency oscillation grows just prior to the transition, and, at least in the upward transition cases, decays over a period of time after the transition. These frequency beats are due to periodic pulling. Compare this behavior to the instantaneous frequency behavior of mode transitions caused by changes in the discharge current, shown in Figure 3.11. The frequency oscillations shown in this figure, from Weltmann et al. (2000), show similar qualitative behavior. These undriven mode transitions reflect periodic pulling of the two modes (the subdominant mode and the dominant mode, as they trade roles) on each other.

The easiest way to recognize dynamics modulation as it happens in the laboratory is to identify the peculiar spectral signature. As a confirmation, the amplitude of the oscillation usually differs between the two modes, and this can be seen on the oscilloscope as well. 


\section{Chapter IV}

\section{Experimental Results}

In this chapter, experimental data collected during the dynamics modulation study is presented. The first section includes basic behavior of the discharge modes as a function of discharge current. The second section describes Arnol'd tongue boundaries for four separate discharge currents. Two of these, for adjacent modes, were chosen to be centered between currents where upward and downward mode transitions would occur. The other two are positioned near, but just inside, upward and downward mode transitions. Finally, the occurrence of dynamics modulation is shown on the relevant Arnol'd tongue plot. These two sections allow a direct comparison to Figure 2.10, the dynamics phase diagram of Weltmann et al. (2000).

The third section presents, in detail, data from the dynamics modulation observed in this study. This section focuses on dynamics modulation due to a driving force near the next higher mode or "upward modulation." Dynamics modulation between the same modes of Weltmann, et al. is shown as a function of driving frequency with example time series, instantaneous frequency series, and power spectra.

Next, methods for extracting the entrainment parameter $\alpha$ and the normalized driving amplitude $M$ from the time series data are extended to the dynamics modulation case for both the temporal and the spatiotemporal parts of the modulation. These methods are equivalent to the method of Chapter III in which the beat frequency $\Omega$ was measured directly from the instantaneous frequency series or as sideband spacing on the FFT. These are used to show the behavior of $\alpha$ and $M$ versus driving frequency for the two parts of the modulation. A close look at the relative phase between the time series and the driver comes at the end of the third section. 
SECTION A. MODES IN THE DISCHARGE

Downward dynamics modulation, which occurs when the driving force is near the next lowerfrequency mode, has not been previously documented, but is presented here. This behavior was not observed to occur in the periodic manner that upward case did, and seemed to have two varieties, which are presented in the last section of this chapter.

\section{A. Modes in the discharge}

The modes observed in the neon glow discharge tube are presented in Figure 4.1 as a function of discharge current. Individual modes appear as a band of triangles stretched relatively horizontally across a range of discharge currents. Each individual mode is identified by a number, written with a preceding $f$, in order of increasing frequency. Modes are identified by these labels throughout the rest of this thesis. On the figure, the modes involved in the dynamics modulation studied, $f 6$ through $f 9$, are identified by labels.

As is true of any series of $p$-type ionization waves, these exhibit hysteresis as a function of current. At any given current, one mode will dominate. Which mode that is depends on the way the current is approached. On the figure, mode frequencies measured following an increase in current are indicated with triangles pointing up. Those measured following a decrease in current are shown with triangles pointing down. The mode transition currents are shown with solid lines. For each pair of lines, the right line indicates the mode transition current while increasing the current and the left line indicates the mode transition current while decreasing the current. Transition current values are very reproducible.

An example of an undriven striation wave mode frequency is shown in Figure 4.2. There is little uncertainty about the dominant frequency for this data, as the spectrum is very narrowly peaked. A different perspective is gained from the close-ups of Figure 4.3. These four plots show the same data as Figure 4.1, but emphasize the variation in mode frequency for each of the four highest modes. Note that each mode follows a concave-up curve, with the minimum frequency representing a central current value, relatively far from mode transitions.

In addition to the overall curve, there is an apparent offset between the left and right halves of several of the curves. These differences are the result of the discharge's history. When two values appear for one current, the lower one was obtained while the current was increasing, while the 
higher was achieved while the current was decreasing. In addition to system history, the presence of a resonant laser beam can alter where the narrow peak occurs by several $\mathrm{Hz}$ [ Weltmann et al. (2000)].

If used with these limitations in mind, Figure 4.1 can serve as a tool for estimating the mode frequency as a function of discharge current. Minimum and maximum frequencies derived from the plot are summarized in Table 4.1, along with frequency ranges for each mode and frequency separation between modes.

\section{B. Arnol'd tongue boundaries}

The method for determining Arnol'd tongue boundaries was presented in Chapter III. Here, the results of this process are presented.

\section{Arnol'd tongue diagrams where one mode dominates}

Using Figure 4.1, the value of discharge currents optimal for modes $f 7$ and $f 8$ were empirically determined by identifying the midpoint of the $f 7$ stair step and the $f 8$ stair step, i.e., as far as possible from the mode transitions on either side. These currents were $9.10 \mathrm{~mA}$ and $11.37 \mathrm{~mA}$. The Arnol'd tongues for these currents are presented in Figures 4.4 and 4.5. To show how these two Arnol'd tongues overlap, they are presented together in Figure 4.6.

The dominant mode is immediately clear in each case as the one whose Arnol'd tongue extends downward to a very narrow tip. This Arnol'd tongue tip can be imagined to extend down to the axis from this narrow tip to the natural frequency, which can be thought of as the frequency that results from the limiting case of a zero-amplitude driving force. At this current, the lowermost tip of the the Arnol'd tongue diagram would intersect the frequency axis at $1450 \mathrm{~Hz}$ in Figure 4.4 and at $1721 \mathrm{~Hz}$ in Figure 4.5, the measured natural frequencies.

The modes to the left and right of the dominant modes are clearly visible in these Arnol'd tongue diagrams. These neighbor modes, however, do not achieve non-negligible amplitude at these currents without being driven by the laser. Again, these currents were selected so that one mode would dominate the undriven discharge tube. 


\section{Arnol'd tongue boundaries near mode transitions}

Figure 4.7 shows two more Arnol'd tongue boundaries, this time at currents within the range of $f 7$, but near the transition currents to lower and upper modes. The currents were arrived at by starting in mode $f 7$, and then adjusting away from the center of that mode toward the transition current, stopping before the transition occurs. These currents, therefore, are currents at which two modes are possible depending on the hysteresis. The currents selected were $8.23 \mathrm{~mA}$ and 10.45 $\mathrm{mA}$, near the transitions from $f 7$ to $f 6$ and $f 8$, respectively.

While locating Arnol'd tongue boundaries, the laser chopping frequency could be used to select which of the two modes would dominate in each case. In Figure 4.7, the $f 7$ mode (at $1462 \mathrm{~Hz}$ for the lower current and at $1447 \mathrm{~Hz}$ for the higher current, both values consistent with Figure 4.3) remains the longest because the currents were slightly on the $f 7$ side of the transition. But the $f 6$ mode (at $1212 \mathrm{~Hz}$ for the lower current value) and the $f 8$ mode (at $1726 \mathrm{~Hz}$ for the higher current value) extend down to sharp points as well, different from their behavior in Figures 4.4 and 4.5. This is because these two modes can occur as natural undriven modes at these currents, even though $f 7$ is slightly favored.

\section{Occurrence of dynamics modulation}

Testing the mechanism published by Weltmann et al. (2000) requires identification of the locations on the Arnol'd tongue diagram at which dynamics modulation occurs. In order to do this, dynamics modulation was studied for the two values of discharge current depicted in Figures 4.4 and 4.5. These currents were chosen to be in the heart of their respective modes ( $f 7$ and $f 8)$, equidistant from the upward and downward transition currents.

Dynamics modulation similar to that documented by Weltmann, et al. was seen in both cases. From now on, this type of dynamics modulation is referred to as "upward dynamics modulation." This refers to the relative frequencies of the driver and the natural mode. The driving frequency is above the natural mode so that dynamics modulation occurs as temporary excursions upward from the normally dominant mode. Downward dynamics modulation was also seen, but differed in important ways from a mirror-symmetric version of the upward case. These cases are described in the last section of this chapter. 
Figures 4.8 and 4.9 show where dynamics modulation was observed relative to the Arnol'd tongues already presented for these two discharge currents. Each triangle represents an instance of observed dynamics modulation. The triangles appear in two clusters on each figure. The right cluster represents cases of the upward dynamics modulation, while the left cluster represents downward dynamics modulation.

These black triangles in the experimental data (Figures 4.8 and 4.9) can be compared directly to the conceptual analog of Weltmann et al. (2000) (Figure 2.10). The right-hand cluster of black triangles in the data represent the open circle and open square on the hypothetical map. While there are some differences between the oversimplified conceptual map and the experimentally documented map, e.g., the shape of the boundary, qualitative similarity is clear. This comparison and its implications for evaluating the proposed mechanism are explored further in Chapter VI.

\section{Upward dynamics modulation}

\section{Time series data versus driving frequency}

Once the characteristic signatures of the dynamics modulation phenomenon were reproducibly observed, more convincing evidence was collected from high-time resolution time series for a series of driving frequency values. Representative highlights from one of these data sets is shown in Figures 4.10 through 4.12. This data was collected at a discharge current of $9.10 \mathrm{~mA}$. The driving frequency, from top to bottom, was 1681 (a), 1688 (b), 1696 (c), 1704 (d), 1710 (e) and 1713 (f) Hz. Entrainment occurred at $1712 \mathrm{~Hz}$.

In Figure 4.10, six examples of the unprocessed time series are presented. In Figures 4.11 and 4.12, the corresponding instantaneous frequency series and power spectra, respectively, are shown. The individual oscillations are obscured in the time series view, but dynamics modulation appears as non-periodic ( $\mathrm{b}$ and e) and periodic (c and d) variations in the amplitude. This identification of the dynamics modulation is confirmed by comparing the instantaneous frequency series and power spectra to the signature of dynamics modulation presented in Figure 3.10.

The top example, (a), shows spatiotemporal pulling, which can be identified by comparing its signature to that shown in Figure 3.8 and the bottom example, (f), shows entrainment, which indicated by its lack of frequency and amplitude modulation and its single-frequency spectral peak. 
Upon closer examination of the instantaneous frequency series shown in Figure 4.11, the behavior seen in the dynamics modulation can be split into three distinct categories. This was true of all of the upward dynamics modulation seen in this study. The first category is periodic dynamics modulation (PDM), already mentioned as being present in (c) and (d). The aperiodic dynamics modulation of (e), however, appears different from that of (b) in that it seems to spend irregular amounts of time entrained to the higher mode before modulating back to the lower mode. This behavior was categorized as dynamics modulation with entrainment (DM/E), while behavior such as (b) was categorized as aperiodic dynamics modulation (ADM). These three dynamics categories, along with spatiotemporal periodic pulling (ST) and entrainment (E), constitute five categories of behavior that were observed in these data sets.

This representative sequence of dynamics modulation data versus driving frequency was selected from a data set of 68 files. The conditions under which this data was collected, as well as the conditions for the other two major data sets used in this study, are summarized in Table 4.2.

\section{Extracting $\alpha$ and $M$ from time series data}

The usual method for extracting $\alpha$ and $M$ is to identify the beat frequency $\Omega$ from the experimental data, use $\Omega$ to determine $\alpha$ from Eq. (2.22) and the reference beat frequency $\Omega^{\prime}$, then use $\alpha$ to find the normalized driving amplitude $M$ using Eq. (2.18). In the temporal pulling case, $\Omega$ could be found from the period of the frequency oscillations in the instantaneous frequency series, or, equivalently, by identifying the sideband separation in the power spectrum. For the spatiotemporal pulling case, using sideband separation is particularly straightforward because the sidebands are prominent and widely spaced.

In the case of dynamics modulation, this is more difficult due to the combination of temporal and spatiotemporal pulling that happen alternately, creating a hybrid power spectrum. Also, the sideband separation is expected to be very small in the temporal case because significant pulling exists and is limited to a very small frequency range.

To determine the beat frequency, the fact is exploited that the frequency separation between spectral features in the FFT is approximately equal to the beat period. This is demonstrated in Figure 4.13. For the two temporal cases shown, the sideband separation was given in the discussion of Figure 3.7 as $35.8 \mathrm{~Hz}$ (top) and $41.6 \mathrm{~Hz}$ (bottom). In the expanded view of Figure 4.13, it is 
clear that an alternate method of identifying this separation is to look for a separate peak in the power spectra located at the separation frequency value. These peaks are seen at $36 \mathrm{~Hz}$ (top) and $41 \mathrm{~Hz}$ (bottom). Some precision is lost, due to the $1 \mathrm{~Hz}$ resolution of the power spectrum, but the associated uncertainty is comparable to the systematic, $\alpha$-dependent error corresponding to treating the time-averaged spectrum as a surrogate for the instantaneous dynamics. A simple estimate of the error bar for $\Omega_{T}$ can be derived from the width of the peak corresponding to sideband separation. And, in cases of aperiodic dynamics modulation, this method yields an average temporal pulling value that would be difficult to ascertain otherwise.

A careful glance at Figure 3.10 makes clear that this algorithm is valid in dynamics modulation cases. There is a clear peak at $21 \mathrm{~Hz}$, a value that can be seen to match the separation of the closelyspaced sidebands. This method makes obvious the interpretation of the "hair" on the signature dynamics modulation spectrum. These narrowly spaced features result from temporal periodic pulling during the upper part of the dynamics modulation cycle. As the driving frequency gets closer to the higher mode frequency, the spacing between these features diminishes, as can be seen in Figure 4.12.

In the spatiotemporal case, the modified beat frequency $\Omega_{S T}$ is calculated as the instantaneous beat frequency of the time series similar to the way $\Omega$ was calculated from the instantaneous frequency series during the discussion of Figure 3.6. This is done by placing a horizontal line through the instantaneous frequency series so that the frequency oscillations in the lower (and, for a second line, upper) part of the modulation cross it periodically (Figure 4.14). The value of this line is subtracted from the instantaneous frequency series, so that "zero crossings" can then be tabulated and used as is input into the usual instantaneous frequency routine. The result is filtered to exclude false high frequencies resulting from data noise and false low frequencies resulting from data gaps during time spent in the opposite mode. The process produces a filtered array. The median value of the lower array can be identified as $\Omega_{S T}$ for this pulling.

An example of this process is shown in Figure 4.14. The top panel shows the instantaneous frequency series with two lines placed at the values subtracted to identify the "zero" crossings. The bottom panel shows the resulting beat frequencies. These beat frequencies are clearly bunched in two groups, coinciding with the two parts of the dynamics modulation cycle. The dashed lines represent the median values of the two measured beats. The lower values $(\bullet)$ can be identified as 
$\Omega_{S T}$ for the spatiotemporal pulling of the lower mode by the entrained, subdominant upper mode as described in the proposed mechanism. The meaning of the upper values is described in Chapter VI.

When these methods are applied to the data, reproducible values for $\Omega_{S T}$ and $\Omega_{T}$ are produced. This is demonstrated in Figure 4.15. These data come from two data sets taken on two different days, including the data highlighted in Figures 4.10 through 4.12. This upward dynamics modulation occurred between modes $f 7$ and $f 8$. In the top panel, the spatiotemporal beat frequencies are displayed as a function of offset from entrainment (entrainment frequency minus driving frequency). It should be emphasized that these measured values represent the same case of dynamics modulation, with the spatiotemporal (temporal) value representing the time spent in the lower (higher) frequency part of the cycle. The dotted line shows the conventional beat frequency $\left(\Omega_{0}\right)$ and the dashed line shows the reference beat frequency $\left(\Omega^{\prime}\right)$. Values from the two days overlap quite well. Near the entrainment end, three data points exceed the reference beat frequency, causing the calculation of $\alpha$ to fail in these cases.

In the lower half of Figure 4.15, reproducible values of $\Omega_{T}$ for the same data are displayed. Again, the dotted line shows the conventional beat frequency $\left(\Omega_{0}\right)$ and the dashed line shows the reference beat frequency $\left(\Omega^{\prime}\right)$. However, in this presentation, since the driving frequency is near the spontaneous mode frequency, the two lines overlap. Representative error bars are indicated for both halves of the figure.

\section{Results of the $\alpha$ and $M$ calculations}

The resulting calculations of $\alpha$ and $M$ from the $\Omega$ values displayed in Figure 4.15 are shown in Figure 4.16 for the two portions of the dynamics modulation cycle. Again, the $x$-axis displays the offset from entrainment. The solid lines to the right (and near the top of the temporal $\alpha$ subplot) indicate entrainment to the $f 8$ mode. Note that just as the system reaches the point where it permanently entrains to the upper mode, $\alpha_{T}$ climbs to one. The value of $\alpha_{S T}$ remains relatively constant throughout the range of dynamics modulation, while $M_{S T}$ climbs slowly and $M_{T}$ declines slowly as entrainment is approached.

The corresponding plot for dynamics modulation between the next higher pair of modes ( $f 8$ and $f 9)$ appears in Figure 4.17. The axes and other formatting of this figure is otherwise identical 
to Figure 4.16. It is immediately clear that the trends of the data reproduce the earlier case, though the values themselves differ somewhat.

The $M$ values of Figure 4.16 are displayed as a ratio of $M_{S T} / M_{T}$ in Figure 4.18. Again, it is clear that the data from the two days are consistent. The ratio of the two normalized driving amplitude values increases from around one to around three as entrainment is approached. The corresponding plot for the $M$ values of Figure 4.17 is shown in Figure 4.19. These results will be useful in evaluating the proposed dynamics modulation mechanism of Weltmann et al. (2000) and will be revisited in Chapter VI.

\section{Detailed examination of mode behavior}

In order to check some of the predictions of the dynamics modulation model, it is necessary to look closely at the phase evolution between the glow discharge and the driver during the two parts of the dynamics modulation cycle. That is done in four figures in this section. In each, a short segment of time series is expanded and displayed alongside the square-wave signal that was used to control the acousto-optic modulation that chopped the laser light on and off. Each of the four figures also contains a close-up view of the instantaneous frequency corresponding to the segment of time series shown. The four figures are representative cases of the two parts of the dynamics modulation cycle for two separate driving frequency cases.

Figure 4.20 is the first of these close-ups. It represents the lower frequency portion of periodic dynamics modulation with a driving frequency $\left(f_{i}\right)$ of $1690 \mathrm{~Hz}$. This segment of the modulation begins with a discontinuity in the time series at $t=0.2270 \mathrm{~s}$, and ends at $t=0.2507 \mathrm{~s}$, as indicated. (Error bars are estimated to be $\pm 0.0004 \mathrm{~s}$, which is approximately a half-period of the lower frequency oscillation.) During the time spent in the lower mode, the amplitude beats with a period of $0.00457 \mathrm{~s}$.

Figure 4.21 shows a representative example of the corresponding higher frequency portion of the same dynamics modulation time series. This segment of the modulation begins when the time series and driver quickly change from out-of-phase to in-phase at $t=0.0124 \mathrm{~s}$, and ends with the opposite change at $t=0.0329 \mathrm{~s}$. During the time spent in the higher mode, the amplitude beats with a period of $0.00430 \mathrm{~s}$.

Figures 4.22 and 4.23 show representative portions of the periodic dynamics modulation cycle 
for a higher driving frequency of $1700 \mathrm{~Hz}$. In Figure 4.22, the beginning of the lower frequency part of the cycle begins when the phase of the luminosity signal changes quickly at $t=0.2842 \mathrm{~s}$, and ends at $t=0.3071 \mathrm{~s}$. (The signal almost snaps out of its lower mode at $t=0.3031 \mathrm{~s}$, but appears to remain for one more beat. That it remains in the lower mode is more clear when this figure is compared to the higher frequency part of the cycle in Figure 4.23, which shows much less relative amplitude modulation.) During the time spent in the lower mode, the amplitude beats with a period of $0.00483 \mathrm{~s}$.

Figure 4.23 completes the series. Here, the higher frequency part of the cycle begins at $t=0.0108$ $\mathrm{s}$, and ends at $t=0.0400 \mathrm{~s}$. During the time spent in the higher mode, the amplitude does not beat in as obvious a manner as the example shown in Figure 4.21 for a lower driving frequency, but the instantaneous frequency shows seven beat periods.

These four figures are discussed during the evaluation of the mechanism in Chapter VI.

\section{Downward dynamics modulation}

Weltmann et al. (2000) published two representative cases of what has been referred to here as upward dynamics modulation. This means that the undriven state of the glow discharge system is a lower frequency mode, while the driving frequency and the mode visited during the dynamics modulation process are relatively higher.

During the experimental investigation, examples of dynamics modulation were observed that had the opposite relative frequency positions. In these cases, the driving frequency was set near the next lower mode frequency, and the system appeared to undergo downward dynamics modulation. One representative example of this is shown in Figure 4.24. The time series (top), instantaneous frequency (center) and power spectrum (bottom) of downward dynamics modulation are shown. The driving frequency $\left(f_{i}\right)$ was $1200 \mathrm{~Hz}$. The modulation is taking place between the $f 7$ and $f 6$ modes. The discharge current was set to $9.10 \mathrm{~mA}$.

The experimental parameters associated with downward examples of dynamics modulation were recorded so that this variety could be explored further. However, despite a thorough search, no case of periodic dynamics modulation as clear as the examples of upward dynamics modulation presented was found. This is likely due to the difference in the relative location of the two types 
of dynamics modulation on the Arnol'd tongue diagrams (Figures 4.8 and 4.9). The upward dynamics modulation occurs on the side of the Arnol'd tongue of the neighboring mode nearest to the spontaneous mode frequency of the discharge, while the downward dynamics modulation cases were only observed on the far side of the Arnol'd tongue of the neighboring mode. Recall that all locations outside the Arnol'd tongue had to be visited to make the Arnol'd tongue diagram, so the absence of dynamics modulation can be stated with confidence.

The cases of downward dynamics modulation seemed to have two general categories. These are shown in the instantaneous frequency and power spectra plots of Figures 4.25 to 4.28. As was done in the upward dynamics modulation case, the various series are shown as a function of driving frequency.

A representative sequence of instantaneous frequency series and power spectra are presented in Figures 4.25 and 4.26, respectively. Beginning at the top, the driving frequency $\left(f_{i}\right)$ is 1385 (a), 1395 (b), 1403 (c), 1405 (d), 1406 (e) and 1409 (f) Hz. The modulation took place between the $f 7$ and $f 6$ modes, and the discharge current was set to $9.10 \mathrm{~mA}$. Entrainment occurred at $1409 \mathrm{~Hz}$ for this series, for which the laser wavelength was set to $588.351247 \mathrm{~nm}$.

None of the instantaneous frequency series shown in Figure 4.25 display the periodic mode switching commonly found in the upward cases. (See Figure 4.11). Figure 4.25a appears roughly periodic, but rather than alternating between spatiotemporal and temporal pulling, it appears to simultaneously undergo both processes. Occasional dynamics modulation to the lower mode appears in Figure 4.25b-e, but not periodically. Throughout Figure 4.25f, entrainment is complete.

The corresponding power spectra, shown in Figure 4.26, do not resemble the signature dynamics modulation spectra of the upward cases. The first case, Figure 4.26a, is most notable because it also seems to represent simultaneous temporal and spatiotemporal pulling signatures.

The second category of downward dynamics modulation is shown in Figures 4.27 and 4.28. As before, a sequence of instantaneous frequency series and power spectra are shown as a function of driving frequency. Beginning at the top, the driving frequency $\left(f_{i}\right)$ is 1420 (a), 1423 (b), 1426 (c), 1429 (d), 1432 (e) and 1435 (f) Hz. The modulation also took place between the $f 7$ and $f 6$ modes. The discharge current was again set to $9.10 \mathrm{~mA}$. Entrainment occurred at a higher driving frequency, at $1435 \mathrm{~Hz}$, for this series, because a longer wavelength (i.e., lower driving amplitude, see Figure 3.2) of $588.351547 \mathrm{~nm}$ was used. 
The first three series shown (Figures 4.27a-c and 4.28a-c) resemble Figure 4.25a somewhat, and even seem to undergo brief dynamics modulation excursions to the lower $(f 6)$ mode. But as the driving frequency is increased, the system undergoes a mode transition that does not reverse. Consequently, the behavior of $4.27 \mathrm{~d}$-e and $4.28 \mathrm{~d}$-e is simple temporal periodic pulling. The power spectra in Figure 4.28 confirm this.

The case of downward dynamics modulation, with its qualitatively different behavior both in the time series analysis and in its occurrence on the Arnol'd tongue, is discussed in Chapter VI. 


\section{Chapter V}

\section{Modeling}

In this chapter, a model is described and used to reproduce some of the behavior seen the in the dynamics modulation experiment. Two coupled van der Pol equations are used to model the mechanism described by Koepke, Weltmann, and Selcher, and the resulting time series is solved with a Runge-Kutta routine whose parameters can be adjusted as the simulation proceeds. Quantitative comparison between experimental observations and the numerical model further establishes the validity of the mechanism.

\section{A. Description of the model}

\section{Requirements}

A useful model of dynamics modulation must accomplish several basic tasks. First, the model must be able to reproduce more fundamental behaviors that have already been published. These behaviors include periodic pulling, both weak and strong, and entrainment. Second, the model should be able to handle two normal modes at once as well as a driving frequency that represents the laser. Third, the model should allow a systematic exploration of parameter space to look for prime locations for dynamics modulation and to ensure that the model's behavior, as implemented, remains consistent with the experiment. To accurately characterize behavior for this use, the model should be allowed to run with constant parameters until a final steady state behavior is recorded.

In addition, a fourth requirement is necessary. To contribute to the evaluation of the mechanism of Koepke, Weltmann, and Selcher, i.e., the purpose of this thesis, the model must be able to adjust 
its input parameters in response to its output parameters. This is implemented in a slightly modified version of the steady state model which periodically measures the average of recently acquired instantaneous frequency values to detect a mode transition. When a mode change is detected, the input representing the normalized driving amplitude is adjusted in a manner consistent with the experimental results of Chapter IV.

\section{Coupled van der Pol equations}

There is no a priori reason to use one particular model over any other model. Since the 1D van der Pol equation (Eq. (2.1)) has been used successfully to model both weak and strong periodic pulling and entrainment [e.g. Koepke and Hartley (1991) and Klinger et al. (1995)], the van der Pol equation will be used as the starting point for this model.

To accommodate two competing normal modes in the discharge, two coupled van der Pol equations were chosen. However, many published studies of the nonlinear behavior of coupled van der Pol equation [for example Pastor et al. (1993) and Pastor-Díaz and López-Fraguas (1995)] do not include driving terms, a requirement for this thesis.

This is also true of van der Pol's own coupled example, which was the first multifrequency oscillator described [ Lamb Jr (1964)]. Van der Pol described his oscillator as a "triode oscillator with two degrees of freedom," but showed it had a property that fits our application of the multimode discharge tube [van der Pol (1934)]. This property is that the solution in which "both oscillations would be present simultaneously [...] is shown to be unstable." This property matches the behavior of the undriven discharge tube well, which strongly favors one mode at a time.

His model, in his original notation, is:

$$
\left\{\begin{array}{r}
\frac{d^{2} v_{1}}{d t^{2}}-\alpha_{1}\left(1-v_{1}^{2}\right) \frac{d v_{1}}{d t}+\omega_{1}^{2} v_{1}+k_{1} \omega_{1}^{2} v_{2}=0 \\
\frac{d^{2} v_{2}}{d t^{2}}+\alpha_{2} \frac{d v_{2}}{d t}+\omega_{2}^{2} v_{2}+k_{2} \omega_{2}^{2} v_{1}=0
\end{array}\right.
$$

Here, $v_{1}$ and $v_{2}$ represent the time-dependent outputs of the two oscillators and $\omega_{1}$ and $\omega_{2}$ the two mode frequencies. His $\alpha_{1}$ takes the place of $\epsilon$ in Eq. (2.1), and $k_{1}$ and $k_{2}$ represent the coupling terms. Notice that the first equation effectively sets $\beta$, the coefficient of $v_{1}^{2}$, equal to one while second equation lacks $\beta$ altogether and differs in sign before $\alpha_{2}$, making it a conventional damped, 
harmonic oscillator.

To adapt this set of equations to the present purpose, several changes are made. First, forcing terms must be added so that it can describe two discharge modes competing in a driven environment. Next, the two normal modes that compete in the driven discharge tube can be expected to have similar behavior in most parameters except mode frequency, so the sign is changed on $\epsilon(\alpha$ in van der Pol's notation) and $\beta$ is added to the second equation, making the forms symmetric. (Recall that van der Pol did not have our access to numerical methods or he might have done this himself.) The notation is modeled after Lashinsky (1968), with the addition of $\gamma_{1}$ and $\gamma_{2}$ in the place of the coupling constants $k_{1}$ and $k_{2}$. Finally, substitutions are made to create a system of four, first-order differential equations suitable for numerical analysis. The result is

$$
\left\{\begin{array}{r}
\frac{d x}{d t}=y \\
\frac{d y}{d t}=\frac{1}{2} \omega_{1}^{2} M_{1}\left[1+\operatorname{sign}\left(\cos \omega_{i} t\right)\right]+\epsilon_{1}\left(1-\beta_{1} x^{2}\right) \omega_{1} y-\omega_{1}^{2} x-\gamma_{1} \omega_{1}^{2} z \\
\frac{d z}{d t}=v \\
\frac{d v}{d t}=\frac{1}{2} \omega_{2}^{2} M_{2}\left[1+\operatorname{sign}\left(\cos \omega_{i} t\right)\right]+\epsilon_{2}\left(1-\beta_{2} z^{2}\right) \omega_{2} v-\omega_{2}^{2} z-\gamma_{2} \omega_{2}^{2} x
\end{array}\right.
$$

Here, $x$ and $z$ take the place of van der Pol's $v_{1}$ and $v_{2}$ as the time-dependent values of the two oscillators. The sum of $x$ and $z$ was taken to represent the combined behavior of the system, equivalent to the output of the photodiode detector in the experiment. The sign-function form of the driving term creates a square-wave force that alternates between $100 \%$ and $0 \%$ to model the driving force of the chopped laser light.

\section{Step-by-time-step solution}

Once the coupled equations are expressed in the form of a system of first-order ordinary differential equations, the problem is reduced to solving them. To do this, the common fourth-order RungeKutta routine [Boyce and DiPrima (1986)] is used. The basic routine (see Appendix B. for the code) required as inputs the beginning and ending of the time interval, the number of steps, the initial conditions, and the equation parameters. The model outputs an array of time steps and a matrix that included values for $x, y=d x / d t, z$, and $v=d z / d t$ for each time step. 
A separate function routine was used to provide the inputs to the model, periodically examine the output, change the inputs if necessary while keeping the time steps even and the output continuous (i.e., it used the output of the last time step as the initial conditions for the next time step when changes needed to be made). These routines were used to create the various trend plots and plots of dynamics modulation displayed in this chapter. An example of one of these control functions is shown in Appendix B..

\section{Handling mode transitions}

The model was designed to pause frequently and perform calculations on the output designed to detect a change in the dominant mode. The pauses occurred after each period of only $0.1 \mathrm{~ms}$ of simulated time, the routine calculated the instantaneous frequencies of the time series up to that point. If the median value of the most recent 25 instantaneous frequency results was above (below) a set threshold, the high (low) frequency mode was detected. Since the high mode frequency was set to be $1740 \mathrm{~Hz}, 25$ instantaneous frequencies would occur in a minimum time of approximately $14 \mathrm{~ms}$. The median instantaneous frequency over at least that amount of time would have to pass the threshold to claim the occurrence of a mode transition. This prevented outlying instantaneous frequency values from being identified as a mode transition.

The threshold above or below which the median instantaneous frequency value had to fall to detect a mode transition was set to be be unambiguous. To detect the high (low) mode as dominant, the median instantaneous frequency had to be above (below) the mean frequency between high and low plus (minus) $35 \%$ of the frequency separation between them.

Two values of the absolute driving amplitude $M$ were used in the model. The specific $M$-value used depended on which mode was detected. In the mechanism, a switch to the higher mode is accompanied by a reduction in $M$. Accordingly, $M_{L}$ and $M_{H}$ were used, with the subscripts $L$ and $H$ representing the alternating dominant modes, low or high frequency, and with $M_{L}>M_{H}$. They were selected to differ by a factor of two, a number that is consistent with the experimental results of Chapter IV (see Figure 4.18).

When a mode transition was detected, the absolute driving amplitude $M$ was toggled to the opposite value in a linear progression over a period of $0.010 \mathrm{~s}$. This time interval is consistent with the time required for a discharge to "settle" into a new mode after a mode transition. [See, for 
example, Figure 3 in Koepke et al. (1996).] During the changing of $M$, mode-transition detection is suspended, but the model otherwise is running in a manner completely consistent with its steady $M$ operation.

\section{B. Identifying experimental parameters}

It is somewhat daunting to search for parameter values associated with a specific behavior amid the many independent parameters of Eq. (5.2). This problem was simplified by restricting the model's use to cases in which $\epsilon_{1}=\epsilon_{2}=\epsilon, \beta_{1}=\beta_{2}=\beta$, and, aside from the toggling with mode changes, $M_{1}=M_{2}=M$. With this starting point, the model was used first in a trial and error manner. This served the simultaneous purpose of lending some intuition about the behavior and limits of the model while the code could be optimized for its intended use.

Promising behavior was quickly seen. For a case in which $M$ was not toggled and for zero initial conditions, the instantaneous frequency was seen to start in a high frequency mode, oscillate with increasing amplitude, rapidly switch to the lower frequency mode, and then settle into that mode with the instantaneous frequency oscillations quickly decaying to zero.

This behavior qualitatively resembled the behavior shown in the bottom half of Figure 3.11 [from Weltmann et al. (2000)], suggesting that the parameters used were relevant to the discharge tube used in this study. Those parameters were $\epsilon=.02, \beta=1, M=.001$, and the driving frequency $\left(f_{i}=\omega_{i} / 2 \pi\right)$ set to $1.17 \times \omega_{1}$, equivalent to $f_{i}=1700 \mathrm{~Hz}$ in a system with $\omega_{1} / 2 \pi \approx 1450 \mathrm{~Hz}$. The coupling parameters were set at $\gamma_{1}=0.1$ and $\gamma_{2}=0.3$. Though there were some differences between the model at this early stage and the final version, the parameters served as a starting point in the search.

\section{Successful examples}

First, as stated above, the new model must be able to handle temporal periodic pulling behavior. This is shown in Figure 5.1. Here, $\epsilon_{1}=0.1, \beta_{1}=1$, and $M=0.03$. The coupling parameters were set at $\gamma_{1}=0.025$ and $\gamma_{2}=0.22$. $\omega_{1}=2 \pi \times 1450 \mathrm{~Hz}$ and $\omega_{2}=2 \pi \times 1740 \mathrm{~Hz}$, the values from the experimental data. For this one case, $\epsilon_{2}=\beta_{2}=M_{2}=0$, to supress the second mode. $f_{i}$ was 1500 (a), 1486 (b), 1472 (c), 1458 (d) 1444 (e), and 1430 (f) Hz. The qualitative behavior of the model 


\section{SECTION B. IDENTIFYING EXPERIMENTAL PARAMETERS}

(Figure 5.1) is very similar to that of the experiment seen in Figure 3.4. Note that the sidebands appear on opposite sides in the manner of Figure 3.7 because the experimental case is being driven at a frequency below its undriven mode frequency while the model case is being driven from above.

The typical experimental case of periodic dynamics modulation was shown in Figure 3.10. This served as a guide as the input parameters to the model were systematically adjusted. This figure shows modulation in which approximately half of the time is spent in each of the two modes. There is a sharp change in the median frequency between the two modes, indicating that the two states are separate.

With the restriction that $M_{L}=2 M_{H}=0.04$ and with $\omega_{1}=2 \pi \times 1450 \mathrm{~Hz}$ and $\omega_{2}=2 \pi \times 1740 \mathrm{~Hz}$, consistent with the experiment, a search was conducted for the best modulation example resulting from $f_{i}=1705 \mathrm{~Hz}$. Success was judged by direct comparison to Figure 3.10. Although a dischargetube-like mode transition was quickly found in the early stages of modeling, it was significantly more difficult to locate examples of dynamics modulation behavior that were good matches to the experimental data in all ways. Some compromise proved necessary. The ways in which the modeled dynamics modulation compares favorably and unfavorably with the experimental results will be discussed as the modeling results are presented, and in Chapter VI.

One result of the search for dynamics modulation in the model is shown in Figure 5.2. For this example $\epsilon=0.1$ and $\beta=1$. The coupling parameters were set at $\gamma_{1}=0.025$ and $\gamma_{2}=0.22$ and the mode frequencies were set at $\omega_{1}=2 \pi \times 1450 \mathrm{~Hz}$ and $\omega_{2}=2 \pi \times 1740 \mathrm{~Hz}$. These values were used throughout the rest of the figures in this chapter, with exceptions for the specific parameter varied in each case. These exceptions are described when each figure is discussed.

While Figure 5.2 is not a perfect match for Figure 3.10, it unmistakably undergoes dynamics modulation. The top panel depicts the resulting time series of the model, or $x+z$ in Eq. (5.2). In the center panel, the instantaneous frequency calculated from the time series is shown, while in the bottom panel, the corresponding power spectrum is shown. The modulation in the frequency in the center panel corresponds exactly to the amplitude modulation in the power series, as we expect for dynamics modulation (see Figures 4.10 and 4.11.) The power spectrum shows a combination of the widely separated sidebands (associated with spatiotemporal periodic pulling) and narrowly separated sidebands (associated with temporal periodic pulling), providing the distinct look of the dynamics modulation spectra. 
If the driving frequency is lowered sufficiently from the dynamics modulation case, the experimental result is that the system undergoes spatiotemporal pulling. If the model accurately reflects experimental behavior, the same result should happen here, and it does. In Figure 5.3, the model was run for $f_{i}=1675 \mathrm{~Hz}$, but with the same inputs as before otherwise. The result is a good example of spatiotemporal periodic pulling from above. This is shown by the concavedownward instantaneous frequency plot (bottom) which corresponds to the time series presented above it. Compare to the opposite case (concave-upward, spatiotemporal periodic pulling from below) shown for experimental data in Figures 3.8 and 3.9.

\section{Characterizing parameters of the model}

In order to demonstrate that the parameters used in the model are sufficiently representative of the experimental data, several power spectra series are presented. In each series, one of the parameters is systematically varied to show how it affects the output of the model. The remaining parameters remain unchanged. For this series of figures, mode transitions were not detected and $M$ was held constant to a central value of 0.03 between the $M_{H}=0.02$ and $M_{L}=0.04$ used in the dynamics modulation simulation. The remaining parameters once again, when not varied, were set to $\epsilon=0.1, \beta=1$, and coupling parameters $\gamma_{1}=0.025$ and $\gamma_{2}=0.22$. The mode frequencies were set at $\omega_{1}=2 \pi \times 1450 \mathrm{~Hz}$ and $\omega_{2}=2 \pi \times 1740 \mathrm{~Hz}$. The driving frequency was set to $f_{i}=1700 \mathrm{~Hz}$.

\section{Varying $M$}

The whole range of $M$ values was scanned in Figures 5.4 and 5.5. In the series of six power spectra presented in Figure 5.4, $M$ was set to 0.0028 (a), 0.0091 (b), 0.0295 (c), 0.0955 (d), 0.3090 (e), and 1.000 (f). Panels (a) and (b) show the type of spectrum that would be expected when the low mode spatiotemporally pulls the high mode down and the driver (in these top two panels, the narrow peak just below the high mode) temporally pulls the high mode, also down. This is the kind of behavior that the mechanism predicts would happen during the higher frequency portion of the dynamics modulation cycle.

In panels (c), (d), and (e), the spectrum shows the higher mode entrained to the driver and in a position to pull upward on the lower mode. In (c), the entrained higher mode is just slightly 
subdominant to the lower mode, the situation that would be expected during the lower frequency portion of the dynamics modulation cycle although the relative power is more even than it would be in the experimental case where the lower mode clearly dominates in power. In (d) and (e), the higher mode is dominant, showing that these values of $M$ are too high for the experimental case. Finally, in (f) the $M$ value is so high that it entrains everything, as would be expected in the experiment for laser wavelengths between the central two triangles of Figure 3.2. The simulated $M$ values used to model dynamics modulation in Figure 5.2 (and other figures later in this chapter) were located at 0.02 and 0.04, which would occur above and below panel (c) on Figure 5.4.

Figure 5.5 represents the instantaneous frequency as a function of $M$ for the same parameters. The simulation was allowed to run until it reached a steady state for each $M$ before instantaneous frequencies were calculated. Instantaneous frequencies were filtered to exclude values above 3000 $\mathrm{Hz}$, to eliminate false high values from rapid zero crossings at very small amplitude. Therefore, the graph expresses the trend rather than the precise instantaneous frequency behavior. The thick, solid line represents the median value of instantaneous frequency at each $M$ value, while the dotted lines above and below it represent the maximum and minimum instantaneous frequency values for each series. The two vertical, dashed lines represent the $M$ values toggled between during the dynamics modulation simulation. The dashed line on the right (left) represents $M_{L}\left(M_{H}\right)$, which was switched to when the low (high) frequency mode was detected. These two values differed by a factor of two, and reproduced dynamics modulation behavior.

\section{Varying $\epsilon$}

The result of varying $\epsilon$ is displayed in Figure 5.6. Here, $M$ was set to 0.03 and $\epsilon$ was set to 0.0100 (a), 0.0251 (b), 0.0631 (c), 0.1585 (d), 0.3981 (e) and 1.000 (f). As $\epsilon$ increases from panels (a) through $(\mathrm{d})$, the nonlinear behavior becomes more obvious. In (a), the only clearly visible features are the two normal modes and the driving frequency between them. As $\epsilon$ is increased sequentially in (b), (c), and (d), the driving frequency gradually entrains the higher mode and more and more sidebands appear. These effects represent nonlinear behavior that is seen in both the theoretical treatment summarized in Chapter II and in the experimental data. Since these values reflect experimental results, $\epsilon$ is chosen in this region as 0.1 .

Since the theoretical treatment requires that $\epsilon$ be much less than one, it is instructive to look 
at the resulting spectra as $\epsilon$ is set to values approaching one in panels (e) and (f). In these two panels, the low frequency mode drifts downward in frequency, as does the higher mode. Sideband behavior also gets increasingly active to a point well beyond the experimental reality. For these two reasons, these values are avoided.

\section{3. $\quad$ Varying $\beta$}

The theoretical treatment requires no limit on $\beta$ to be accurate, so any value may be used. However, in both van der Pol's relevant result that the case of both modes together is not a stable solution of the (undriven) system and the early simulation result in which a spontaneous mode change looked similar to previous experimental results (both described above), $\beta$ was set to one. In Figure 5.7, the spectra are examined as a function of $\beta$ to make sure that this choice is appropriate.

$\beta$ was set to 0.0100 (a), 0.0251 (b), 0.0631 (c), 0.1585 (d), 0.3981 (e) and 1.000 (f) in Figure 5.7. From top to bottom, there is a similar progression to that seen for the lower values of $\epsilon$, namely increasing nonlinear behavior as $\beta$ is increased. As with $\epsilon$, this is shown by the driving frequency entraining the higher mode more efficiently and by more prominent sidebands at higher $\beta$. The over-active sidebands of high $\epsilon$ are absent however, so there is no problem selecting a $\beta$ value of one.

\section{Varying the coupling parameters, $\gamma_{1}$ and $\gamma_{2}$}

The behavior of the simulated dynamics modulation was most sensitive to changes in the coupling parameters. These parameters are also the least constrained by previous numerical modeling and least informed by the known behavior of the experimental system. The parameters that were used, $\gamma_{1}=0.025$ and $\gamma_{2}=0.22$, were chosen because they resulted in the best simulated dynamics modulation behavior when used with the other parameters. Here, the power spectra are displayed as the coupling parameters are changed so that their effect on the simulation can be seen.

In Figure 5.8, a series of six power spectra are shown in which the value of $\gamma_{1}=\gamma_{2}=\gamma$ is increased. This figure shows the effect of increasing the overall coupling from lower values to higher values, representing a progression from weak coupling to strong coupling. The values used for $\gamma$ in this case are 0.001 (a), 0.0608 (b), 0.1206 (c), 0.1804 (d), 0.2402 (e), and 0.3000 (f).

Again, as was seen with $\epsilon$, as coupling is increased, the lower frequency mode's spectral feature 
shifts to lower frequencies. This is most clear in panels (e) and (f). In this case, however, unlike that of $\epsilon$, the higher mode frequency can be seen to shift to higher frequencies as coupling is increased. This is seen in panels (a) through (d). In panels (e) and (f), the two mode frequencies merge into one feature. (Note that the driver frequency stays at $1700 \mathrm{~Hz}$ throughout.)

To show the effect of the relative difference between $\gamma_{1}$ and $\gamma_{2}$, Figure 5.9 plots increasing $\gamma_{2}$ with $\gamma_{1}$ held constant. The value for $\gamma_{1}$ was chosen to be 0.025 , the value that produced the most promising simulated dynamics modulation. $\gamma_{2}$ was set to 0.0003 (a), 0.0602 (b), 0.1202 (c), 0.1801 (d), 0.2401 (e), and 0.3000 (f). This corresponds to $\gamma_{2} / \gamma_{1}$ ranging from 0.01 to 12.0 .

The interesting sideband structure of (c) and especially (d) suggests dynamics modulation. This is an illusion. In the generation of this figure, recall that $M$ is not being toggled, but rather held constant at a value midway between the two used to model dynamics modulation. The instantaneous frequency plots that correspond to these power spectra reveal that dynamics modulation signature is not occurring for these plots.

The difference between $\gamma_{1}$ and $\gamma_{2}$ is the method used in the model to make one mode favored over the other. In other words, in an undriven system, both modes should not be present. Since the behavior that was modeled represented a case in which the lower frequency mode was dominant, $\gamma_{2}$ was made greater than $\gamma_{1}$. This made the model prefer the lower frequency for low values of $M$ (see Figure 5.4). The $\gamma_{2} / \gamma_{1}$ that was used in the simulation was 8.8, placing it between (d) and (e) in this figure but looking more like (e). (A power spectrum representative of this ratio with other parameters the same is shown in Figure 5.7f.)

\section{Dynamics modulation modeling summary}

\section{Upward dynamics modulation}

One requirement of any successful simulation of dynamics modulation is that it is dependent on driving frequency. Using the modeling parameters described above, a series of simulated cases of dynamics modulation were produced as a function of driving frequency. A subset of these simulations are shown in Figures 5.10 through 5.12. The simulated time series are shown in Figure 5.10 and the corresponding instantaneous frequency series and power spectra are shown in Figure 5.11 and 5.12 respectively. 
For these three figures, the driving frequency $f_{i}$ was 1685 (a), 1690 (b), 1700 (c), 1705 (d), 1715 (e), and 1725 (f). $M$ was applied to both oscillators all the time, and set to toggle between 0.02 (when high mode detected) and 0.04 (when low mode detected), $\epsilon=0.1$, and $\beta=1$. The coupling parameters were $\gamma_{1}=0.025$ and $\gamma_{2}=0.22$. The mode frequencies were set at $\omega_{1}=2 \pi \times 1450 \mathrm{~Hz}$ and $\omega_{2}=2 \pi \times 1740 \mathrm{~Hz}$.

In (a) of each figure, the system spends most of its time in a state of spatiotemporal pulling, but on three occasions the system modulates briefly between the modes. This aperiodic dynamics modulation was also seen in the experiment for driving frequencies on the lower edge of the dynamics modulation regime.

As the driving frequency is increased in (b) through (e), periodic dynamics modulation is simulated. As in the experimental case, the period of the dynamics modulation increases as the driving frequency approaches the upper mode frequency. Similarly, a larger proportion of the time is spent in the higher mode with each increase in driving frequency. Finally, in (f), the system remains permanently in the higher mode. Rather than being entrained there, as in the experimental case, the model arrives at a state in which the upper mode is being spatiotemporally periodically pulled downward. This is due to the model being oversimplified. Although $M$ was toggled down with the change in mode frequency, the forcing was still being applied to both oscillators, and thus the lower mode remains influential, unlike in the experimental case. In order for this behavior to better model the experiment, and undergo entrainment, more assumptions would have to be made than are specifically required by the proposed mechanism of Koepke, Weltmann, and Selcher, which focused on changes in the $M$ parameter. Changes in coupling or differences in $M$ for each of the two coupled oscillators (i.e., $M_{1} \neq M_{2}$ ) in Eq. (5.2) may make the result more realistic. However, attempts to make these adjustments did not improve the overall match to experimental dynamics modulation.

The simulated time series shown in Figure 5.10 shows three main differences when compared to the experimental case in Figure 4.10. The most serious difference is that the frequency of modulation, given by the number of excursions into the upper mode over equal time intervals, is much higher in the simulated case. Two other differences are that the mode transitions are more gradual in the simulated case and that the higher overall amplitude occurs during the upper mode in the simulated case but not in the experimental case. This difference is likely the result of the 


\section{SECTION D. DYNAMICS MODULATION MODELING SUMMARY}

simulation not showing entrainment at the higher mode.

When Figures 5.11 and 4.11, the simulated and experimental instantaneous frequency plots, are compared, more qualitative differences are apparent. There is a difference in the relative size of the oscillation amplitude in the lower versus the higher mode, and in the qualitative behavior of the instantaneous frequency oscillation while in the lower mode. These qualitative differences detract from the usefulness of the the coupled van der Pol system as used in this simple manner as a model for the experimental system. It is encouraging, however, to note that the overall behavior is similar, which suggests that the model captures the essence of the physics of the situation.

The power spectra comparison between Figures 4.12 and 5.12 is also encouraging. Again, the final panel (f) of the simulated modulation is not entrained, but rather shows an example of spatiotemporal pulling signature as mentioned above. The rest of the spectra, however, display the narrowing of the separation of the small spectral features as the driving frequency is increased, similar to the experimental case. Again, this suggests that the fundamental physics is captured approximately in this simple model.

\section{The downward case}

The downward case of dynamics modulation, which was shown in Chapter IV to be qualitatively different in the experimental case, would not be expected to differ in this model. The two coupled equations are handled the same way. If the coupling coefficients were flipped, and the two $M$ values toggled in the opposite manner, the downward dynamics modulation case should result.

This is shown to occur in Figure 5.13. This behavior was produced with the same $\epsilon(=0.1)$, $\beta(=1)$, and coupling parameters $\left(\gamma_{1}=0.025\right.$ and $\left.\gamma_{2}=0.22\right)$ as the upward case. The frequency offset was smaller ( $5 \mathrm{~Hz}$ compared to $40 \mathrm{~Hz}$ ) and the $M$ values were larger ( $M$ was toggled between 0.28 when the low mode was present and 0.56 when the high mode was present compared to 0.02 and 0.04) than were used in the upward case. The mode frequencies were set at $\omega_{1}=2 \pi \times 1703 \mathrm{~Hz}$ and $\omega_{2}=2 \pi \times 1455 \mathrm{~Hz}$. The driving frequency, $f_{i}$, was set to $1460 \mathrm{~Hz}$.

This driving frequency is above the mode frequency, which represents the symmetric downward dynamics modulation case not observed to occur in the experiment, so realistic experimental values cannot be used to guide the inputs. Still, this result shows that basic dynamics modulation occurs under the circumstances suggested by the proposed mechanism. A reason why modulation can be 
modeled in this simple manner, but does not occur in the experiment, is suggested in Chapter VI.

\section{Summary of modeling results}

In this chapter, a simple model of dynamics modulation was presented, consisting of two coupled van der Pol oscillators. After some trial and error, a starting point for useful parameter inputs was located, which allowed a systematic exploration of parameter space to locate the most realistic combination of parameters.

Simplifying assumptions were made that two oscillators would adequately model the dynamics and that they would share the same $\epsilon$ and $\beta$ parameters. It was assumed that the normalized driving force amplitude $M$ would act on both oscillators equally. Mode detection was used to

model the mechanism of Koepke, Weltmann, and Selcher, [Weltmann et al. (2000)], so that the magnitude of $M$ could be adjusted downward (upward) when the higher (lower) frequency mode was detected.

This model, though simple, captured the essence of the physical situation and led to simulated dynamics modulation that was qualitatively comparable to the experimental case. 
Page is intentionally blank 


\section{Chapter VI}

\section{Discussion}

In this chapter, the results of the experiment are used to evaluate the proposed dynamics modulation mechanism of Koepke, Weltmann, and Selcher [Koepke et al. (1995) and Weltmann et al. (2000)]. This is done point-by-point using the characteristics of the model itemized in Chapter II. The results of the $\alpha$ and $M$ measurements from Chapter IV are central to this process. Using these results, the mechanism is verified.

After this is done, the "extra" beat frequency seen in the data and described in Chapter IV is explained. This additional interpretation is possible because of the new extension of the Lashinsky (1968) periodic pulling treatment described in Chapter II. This result also has implications for mode transitions in undriven discharge tubes, and these implications are discussed.

The model from Chapter $\mathrm{V}$ is discussed next. Although quantitative comparison to the experimental data was not possible with the model, it did reproduce the qualitative behavior of dynamics modulation. Since the model was constructed using assumptions based in part upon the proposed mechanism, its qualitative success can be used to further support the validity of the mechanism.

Finally, the downward modulation case is discussed. An explanation is proposed to explain why it differed qualitatively from the upward modulation case in the experiment. This same explanation also suggests a reason why the model did not fully recreate the richness of the experimental spectrum. 


\section{A. Evaluation of the mechanism}

In Chapter II, an itemized list of predictions derived from the proposed mechanism of Koepke, Weltmann, and Selcher was presented. (See page 26.) At this point, each of these predictions is evaluated in turn using the data from Chapter IV.

\section{Relative reduction in $M$ during the higher-frequency, temporal portion of the modulation}

When $M_{T}$, the $M$ value during the higher frequency, temporal part of the dynamics modulation cycle is compared with the spatiotemporal $M_{S T}$ during the lower frequency part, the result is unambiguous. In Figures 4.16 through 4.19, the two values of $M$ are shown side-by-side, and $M_{T}<M_{S T}$ for nearly every case. The only cases where the data suggest $M_{T} \nless M_{S T}$ are the lowest driving frequency values on Figures 4.16 and 4.18. For these two cases, the dynamics modulation was not yet periodic and the error bars still extend sufficiently to be consistent with the prediction. Therefore, this item of the predicted mechanism is verified.

\section{Subdominant mode alternation between laser entrainment and temporal pe- riodic pulling}

Neon-resonant chopped laser light outside the dominant mode's Arnol'd tongue resulted in a subdominant spectral feature at the chopping frequency along with the appropriate beat-related sidebands. If the driving frequency was changed, the small spectral feature associated with it would also change. This behavior is evidence that the laser entrains subdominant modes easily or, in other words, that the Arnol'd tongues for subdominant modes are much wider than the Arnol'd tongues for dominant modes, such as those shown in Figures 4.4 through 4.7.

This interpretation is further supported by evidence for entrainment during the spatiotemporal part which can be seen in Figure 4.12a. This shows the power spectrum of the system prior to the onset of dynamics modulation. Here, in the vicinity of the expected higher frequency, there is only one feature present. This means that during the lower frequency portion of the dynamics modulation cycle, the subdominant higher mode is entrained.

It remains to show that the system falls out of entrainment during the higher frequency part of 
the cycle. This is shown by parts (b) through (e) of Figure 4.12, in which the driving frequency approaches the higher mode frequency. Narrowly-spaced sidebands appear in these panels, suggesting temporal periodic pulling from a nearby frequency.

Finally, when this narrowly-spaced interval was used to calculate $M_{T}$, with the a priori assumption that it was temporal periodic pulling, it behaved exactly as would be expected. In the top right plot of Figures 4.16 and 4.17, the resulting $\alpha_{T}$ approached one as the entrainment boundary was approached in frequency space. The last value before entrainment (particularly in Figure 4.16) was very close to one, as would be expected for temporal periodic pulling. Therefore, the interaction between the laser light and the higher frequency mode alternates between spatiotemporal (during the low frequency portion of the modulation) and temporal (during the high frequency portion) and this item is also confirmed.

\section{The dynamics phase diagram}

Figure 2.10, from Weltmann et al. (2000), was a prominent part of the explanation of the proposed mechanism. If the proposed mechanism is to be verified, this figure must be compared with the experimentally obtained Arnol'd tongues shown in Figures 4.8 and 4.9.

Figures 4.8 and 4.9 are not exactly comparable to the earlier schematic diagram because they show only the Arnol'd tongue boundaries of the dominant mode. In other words, portions of the Arnol'd tongue that are above the lowest-amplitude boundary can not be mapped using the method described in Chapter II. Another important difference is that the $y$-axis of Figure 2.10 represents $M$ (the normalized driving amplitude) according to the authors, while the $y$-axis of Figures 4.8 and 4.9 is effectively $E$, the absolute driving amplitude.

With these differences in mind, we expect to be able to see points representing Figure 2.10's open circle (o) and open square $(\square)$ representing two cases of dynamics modulation at the comparable location on Figures 4.8 and 4.9. Points do appear as expected, represented as filled triangles ( $\mathbf{\Lambda})$ in several locations. Therefore, this item is also confirmed.

\section{4. $M$ variation is consistent with upper-mode amplitude change}

$M$ is defined (see Eq. (2.8)) as $E / 2 a$. Therefore, the ratio of $M_{S T} / M_{T}$ should be consistent with the ratio of amplitudes $a_{T} / a_{S T}$, where in this case, $a$ refers to the amplitude of the laser-driven 
upper mode during the two parts of the dynamics modulation. This can be checked using the same time-series data used to characterize the dynamics modulation.

To obtain an estimate for $a_{T}$, we need to examine Figure 4.10. In parts (c) and (d) of this figure, the amplitude of the time series changes quite clearly between the two parts of the dynamics modulation. The part needed for $a_{T}$ is the smaller amplitude section (which occurs during the high frequency portion of the modulation as can be seen from Figure 4.11). The amplitude of these parts of the modulation (determined from a magnified view of the data in Figure 4.10) is 0.11 V.

The $a_{S T}$ case is more subtly obtained. To estimate the higher mode's response to the laser light driving during a time when the lower mode is dominant, we can look at the spatiotemporal time series (Figure 4.10a) that occurred at a driving frequency just one frequency step too low to undergo dynamics modulation. Therefore, it has a spectrum uncomplicated by the mode changes of dynamics modulation, but still has a feature that represents the chopped laser light (Figure 4.12a, narrow feature at $1681 \mathrm{~Hz}$ represents the upper, subdominant mode responding to the laser).

To extract the amplitude $a_{S T}$ corresponding to this feature and in units useful for comparison to $a_{T}$ above, a straightforward (if inelegant) method was used. The spectrum of Figure 4.12a was multiplied by a Gaussian centered at $1681 \mathrm{~Hz}$ and with a width of $50 \mathrm{~Hz}$. The resulting spectrum was passed through an inverse Fourier transform routine to produce a time series that consisted of the contribution of this one feature. As a check, the inverse transform of the narrow feature's spectrum, added to the remaining spectral contribution, was also computed, to be sure that it compared favorably with the original data; it did. The resulting estimate of $a_{S T}$, made using the average of data obtained from five spatiotemporal time series was $0.0651 \mathrm{~V}$.

Therefore, the ratio $a_{T} / a_{S T}$ is estimated to be $\sim 0.11 / 0.0651=1.7$. This is consistent with $M_{S T} / M_{T}$ as seen in Figure 4.18, derived from the same data as explained in Chapter IV, in which the ratio spans the range 0.5 through 3.5 depending on driving frequency. This aspect of the proposed mechanism is also confirmed.

\section{The upward mode transition occurs when sufficiently close}

Weltmann et al. (2000) state (pp. 2778-2779) that the upward transition occurs as the instantaneous frequency of the lower mode is pulled sufficiently near the frequency of the higher mode. To address this point, we examine Figures 4.20 through 4.23, which are temporal close-ups of dynamics 
modulation time series along with the driving frequency function, scaled for comparison. We also look at data from the complete $1 \mathrm{~s}$ time series similar to that shown in Figure 4.10.

In Figure 4.20, the system is in the lower mode for most of the frame. Just before the time marked $0.2507 \mathrm{~s}$, the instantaneous frequency climbs very near the higher frequency mode $(\sim 1740$ $\mathrm{Hz}$ ). In a second case, at a different driving frequency and seen in Figure 4.22, the same behavior occurs just before the time labeled 0.3071 s. So the point that the upward transition occurs when the instantaneous frequency pulls sufficiently near the frequency of the upper mode is verified by the data.

\section{The upward mode transition occurs when the lower mode is out of phase with the driver}

Weltmann et al. (2000) also state (p. 2779) that "The upward mode transition takes place when the [higher-frequency] mode, and the chopped light to which it is synchronized, is sufficiently out of phase with the [lower-frequency] mode." For this point, if the upward transition takes place due to the phase relationship of the laser light and the low-frequency mode, that should be discernible from the overall dynamics modulation frequency. If the upward transition only occurs at a certain point in the phase evolution between the chopped laser light and the lower mode, then the periodicity of upward mode transitions should equal the beat frequency between the two modes or a multiple of this beat.

For the cases in Figures 4.20 through 4.23, the upward mode transitions occurred, on average, every $0.0390 \mathrm{~s}\left(f_{i}=1690 \mathrm{~Hz}\right)$ and $0.0414 \mathrm{~s}\left(f_{i}=1700 \mathrm{~Hz}\right)$. This reflects upwards transitions with frequencies of $25.7 \mathrm{~Hz}$ and $24.1 \mathrm{~Hz}$ respectively. The corresponding measured, time-averaged beat frequencies $(\Omega)$ between the chopped-light driver and the lower mode were $219.88 \mathrm{~Hz}$ and 223.36 $\mathrm{Hz}$ respectively. Each reflects an average over all the periods in five $1 \mathrm{~s}$ time series. Therefore, the upward mode transition is occurring, on average, once every 8.56 beat periods, and once every 9.27 beat periods respectively. Since these results are not integer, this analysis is not conclusive regarding the prediction that upward mode transitions involve the relative phase evolution of the chopped laser driver and the lower mode.

The other possible route to evaluating the prediction that the upward mode transition occur at the moment when the laser-entrained upper mode is suitably out of phase with the lower mode is 
by examining the time series close-ups of Figures 4.20 through 4.23 . Keep in mind that the driving frequency series and the photodiode signal may contain a constant arbitrary phase offset in these figures. This is due to the fact that the light signal was collected $57.5 \mathrm{~cm}$ from the cathode, while the laser chopping signal was collected from the signal generator prior to being amplified and sent to the discharge tube, via the AOM at a position $2.5 \mathrm{~cm}$ from the cathode.

Near the beginning of the time series shown in Figure 4.21, the photodiode signal goes from almost exactly out of phase to very nearly in phase in one-half an oscillation cycle. Regardless of the phase offset, somewhere in this interval the two signals would be exactly out of phase. This corresponds precisely to the moment (seen in the bottom panel's plot of instantaneous frequency) that the system transitions to the higher mode. A similar, though less convincing, pattern is clear near the $t=0.0108 \mathrm{~s}$ point in Figure 4.23. Looking at the upward transition at the ends of the low frequency mode close-ups (Figure 4.20 near $t=0.02507 \mathrm{~s}$ and Figure 4.22 near $t=0.3071 \mathrm{~s}$ ) should give similar evidence about the phase condition at the upward transition, but the the evidence is ambiguous.

Therefore, the mechanism item that describes the upward mode transition as taking place when the chopped-light-synchronized high frequency mode is sufficiently out of phase with the low frequency mode has not been conclusively evaluated. The point is plausible but not demonstrated.

\section{The downward mode transition}

The authors further state (p. 2779) that "As the temporal periodic pulling evolves, the phase modulation serves to put back into phase the chopped light and the [higher-frequency mode]. When the chopped light and the [higher-frequency] mode are sufficiently in phase, at which point the laser couples better to the [higher-frequency] mode, the reverse mode transition is induced."

This point suggests that downward mode transitions should occur at periodic intervals equal to (or, possibly, multiples of) the temporal pulling beat frequency $\left(\Omega_{T}\right)$. This can be evaluated by comparing $\Omega_{T}$ as identified from the low-frequency feature of the spectrum (the method described in Chapter IV) to the period of the dynamics modulation, calculated by measuring the period directly from the instantaneous frequency series. The results of this examination are shown in Table 6.1 and Figure 6.1. Since the agreement is excellent, this point is verified. Periodic dynamics modulation occurs at the temporal pulling beat frequency. The two frequencies are identical. 


\section{Reverse evolution of temporal periodic pulling}

The final item of the proposed mechanism that must be evaluated is the authors' statement that the temporal periodic pulling proceeds in the reverse of the usual manner. Typically, temporal periodic pulling starts with the driver and mode in phase, and proceeds to a point where they are sufficiently out of phase that the mode's frequency is no longer effectively pulled and resets to start the cycle again. Here, the authors argue, the opposite is true. They argue that the upper mode is out of phase with the laser chopping frequency at the moment it becomes dominant, and that the modulation returns at the point when the upper mode becomes in phase with the driver again.

It was demonstrated above that the overall dynamics modulation frequency matches the temporal beat frequency. That supports the case that the modulation cycle is connected to the phase evolution of the temporal beat. It is more difficult to establish the direction of phase evolution. The close-up Figures 4.20 through 4.23 can be used to reveal the phase evolution between the signal and the driver at frequencies near the signal frequencies, but are not as useful for illuminating the much slower temporal pulling beat. Therefore, this point remains plausible, but not directly supported.

\section{Verdict on the mechanism}

The mechanism of Weltmann et al. (2000) has been verified in six out of eight itemized points (as described in Chapter II). For the points not fully verified, no data was obtained that was inconsistent with them, leaving them plausible.

It is important to note that the itemized points were not part of the original Weltmann et al. (2000) proposed mechanism but were constructed to guide the testing of the mechanism for this thesis. The points involving the modulation of $M$ (points \#1-4) are the most important parts of the mechanism. These ideas support the alternation between temporal and spatiotemporal behavior, the heart of dynamics modulation. These ideas were all verified in the experimental data.

The remaining points (points \#5-8) involve the phase evolution and the timing of the modulation. Of these, two were verified and two were plausible, but not directly supported. Point \#6 was plausible, but the least likely, with the dynamics modulation frequency matching the temporal pulling frequency and not connected by examination of the data to the phase interaction of the lower mode and the laser-entrained upper mode. The data were consistent with point \#8 being 
true, though this point was not demonstrated in the data.

Since six of the eight points were verified including all of points 1-4 that are most central to the mechanism, the mechanism is verified.

\section{B. Interpretation of the "extra" beat frequency}

\section{The driven case of Chapter IV}

In Chapter IV, a second beat frequency was calculated that differed from the beat between the driver and the low mode (see Figure 4.14). This beat frequency is neatly explained by the extension of the Lashinsky (1968) periodic pulling treatment to cases in which $\omega_{0} \nsim \omega_{i}$.

For $\omega_{0} \sim \omega_{i}$, the conventional beat frequencies between two modes are identical no matter which mode acts as the pulling mode. But for the $\omega_{0} \nsim \omega_{i}$ case, this is not true of the analogous reference beat frequencies. This is shown in Figure 6.2. Here, the dotted lines represent the conventional beat frequency, $\Omega_{0}=\left|\omega_{i}-\omega_{0}\right|$. The reference beat frequency, $\Omega^{\prime}$ (see Eq. (2.20)) is shown with the solid line. For two modes separated significantly, this figure predicts that the reference beat frequency will be higher when the lower-frequency mode pulls the higher-frequency mode than when the roles are reversed. A similar relationship would be expected to be seen for the measured beat frequencies.

It is seen. In the data shown in Figure 4.14, the measured beat frequency for the driver-pullinglow case is at $224.3 \mathrm{~Hz}$ while the low-pulling-high case is measured to be $273.1 \mathrm{~Hz}$. These are compared to reference beat frequencies of $231.6 \mathrm{~Hz}$ and $319.0 \mathrm{~Hz}$ respectively. The theoretical periodic pulling treatment, when extended to the case $\omega_{0} \not \omega_{i}$ as it was in this study for the first time, predicts this difference in beat frequencies.

Using the reference beat frequency calculated for the low mode pulling the high mode, plots complementary to Figures 4.15 (top panel) and 4.16 (left half) can be created. These plots are shown in Figures 6.3 and 6.4. Figure 6.3 shows that the modified beat frequencies measured during the portion of the dynamics modulation in which the upper mode is dominant and pulled downward by the subdominant lower mode (i.e., the low-pulling-high case) are higher for all driving frequencies than the high-pulling-low case (Figure 4.15 top).

Figure 6.4 shows the calculated $\alpha$ and $M$ values for this portion of the dynamics modulation 
cycle. Note that the values of both $\alpha$ and $M$ are significantly higher than those shown in Figure 4.16 (left half), reflecting the system's strong preference for the $f 7$ mode. Still, as entrainment is approached, the values of both $\alpha$ and $M$ trend downward.

\section{General implications}

It was shown in Chapter III that determining $\alpha$ from Eq. (2.10), prior to the extension of the theory to the general case, can be a problem when $\Omega>\Omega_{0}$. (A second glance at Figure 6.2 shows that this is a problem for downward spatiotemporal periodic pulling, as was the case in Chapter III, because on this side $\Omega_{0}<\Omega^{\prime}$.) With the added successful prediction of asymmetric beat frequencies, demonstrated by Figure 4.14, the extended treatment presented in this thesis is established as a useful tool for understanding periodic pulling between widely separated competing modes.

When an undriven glow discharge tube undergoes a mode transition, there is not only a change in mode frequency (and, consequently, instantaneous frequency), there is also a change in instantaneous beat frequency. This is clear from Figure 3.11, from Weltmann et al. (2000). In this figure, the discharge current is constant. [It has also been explicitly depicted, such as in Figure 3 in Koepke et al. (1996).] In the top panel, the mode frequency is increasing, and the beat frequency, seen as the period of the instantaneous frequency oscillations, undergoes an unambiguous increase. In the bottom panel, the opposite occurs. The extended periodic pulling treatment predicts this relative change in beat frequency. Before the mode transition in the top panel, high is pulling low, which has a relatively lower reference beat frequency and therefore a lower observed beat frequency is expected. A future study, in which this possible connection is quantitatively investigated, might prove fruitful.

Another possible application that is worth investigating is the hysteresis shown in Figure 4.1. If mode transitions are indeed caused by spatiotemporal periodic pulling between wave modes, as has been suggested [Koepke et al. (1996)], the extended theoretical treatment and its inherent asymmetry between low and high drivers may help explain hysteresis in mode transitions. In this case, unlike the case described in the paragraph above, the discharge currents are different at the two hysteresis points. Therefore, the mode frequencies themselves may also change (see Figure 4.3). However, as a quick qualitative exploration of this, note that the low-pulling-high reference beat frequency would be larger at any given point than the high-pulling-low reference 
beat frequency. Therefore, when a point is reached where adequate upward pulling is achieved for an upward mode transition, an immediate attempt to reverse the transition would come up short. One would have to adjust the current from that point to a point where the lower mode is more favored, i.e. downward, in order to achieve adequate pulling compared to the higher reference beat frequency. This qualitatively agrees with the extended treatment, and might also prove interesting upon further study.

\section{Discussion of the model}

The model, while not useful for direct quantitative comparison with the experiment, did manage to reproduce the general behavior of dynamics modulation using only an experimentally accurate variation in the $M$ parameter. Since this $M$ variation was a key part of the proposed mechanism, the model results reinforce the experimental verification of the proposed mechanism.

\section{Shortcomings of the model}

The model was only capable of representing two modes, while the experiment had at least nine that may have played some role in the process. It was implemented in a symmetric way $\left(\epsilon_{1}=\epsilon_{2}\right.$ and $\beta_{1}=\beta_{2}=1$ ) that was meant to approximate the real situation but may have had significant shortcomings. The normalized driving amplitude $M$ was applied to both oscillators equally, despite this almost certainly not being true in the experimental case. The mode transition detection algorithm, in order to accurately detect mode transitions, required time that did not accurately reflect the rapid mode transitions of the experiment. These approximations were used due to an absence of direct evidence of better parameters and methods to use, with the benefit of simplifying the model to its essential components.

A direct comparison of Figures 5.10 through 5.12 with their experimental counterparts, Figures 4.10 through 4.12 reveals further shortcomings. As mentioned in Chapter V, dynamics modulation ends not with the upper mode entrained, but with spatiotemporal periodic pulling down occurring constantly. (This was the result of continuously driving both oscillators despite changes in the dominant one.) The amplitude of the time series is larger in the upper mode in the model, where the opposite is true in the experiment. The amplitude change at the mode transition is also 
much more abrupt in the experimental case. The dynamics modulation frequency does not match the experimental case, though the trend is correct. The instantaneous frequency oscillations are different in overall amplitude from the experimental case, and the manner in which their amplitude evolves is qualitatively different as well. The power spectra compare somewhat favorably, but the sidebands appear to be enhanced on the opposite side in the model case compared to the experimental case.

\section{Successful aspects of the model}

Even with these limitations, the model was successful in several ways. First of all, the model exhibits credible dynamics modulation behavior. As driving frequency is increased, dynamics modulation frequency decreases and a larger portion of each cycle is spent in the upper mode. This behavior resulted from just the essential pieces of the mechanism's description, two oscillators, $M$ reduction upon transition to the upper mode, and $M$ increase upon return. The fact that the model captures the essence of dynamics modulation when implemented in this simple manner suggests that the mechanism is correct.

More qualitative agreement can be seen in Figure 6.5. This is the modeled version of Figure 4.14, the experimental case. The driving frequency in this case was set to $1700 \mathrm{~Hz}$. The beat frequency in the low mode (circles) has a median value $(258.6 \mathrm{~Hz})$ that is below that of the high mode's (squares) median $(278.4 \mathrm{~Hz})$. Though the separation is observed to be smaller than in the experimental case, the cycle is similar which further suggests that the model contains true dynamics modulation.

\section{The downward modulation case}

One of the surprises of this thesis was the inability to achieve the symmetric, downward modulation case that would complement the upward modulation first observed by Koepke et al. (1995) and repeated here. As depicted in Figures 4.8 and 4.9, the downward dynamics modulation that was observed occurred on the outside edge of the lower mode's Arnol'd tongue, unlike the upward modulation, which occurred on the inside edge. The model did not explain this difference, and had as much success in the downward symmetric case as it did in the upward case, as was illustrated 
in Figure 5.13.

In order to explain why the symmetric downward case did not occur, and the non-symmetric case did occur, Figure 6.6 was prepared. The bottom panel shows an observed downward dynamics modulation spectrum. For reference, light gray bars are placed at the observed mode frequencies for this discharge tube, and extended into the top panel. Medium gray rectangles are placed at driving frequencies used for dynamics modulation attempts, as labeled. The black rectangles represent the first two expected sidebands opposite the driven mode. In the two successful cases, the first sideband overlaps an existing mode. For the symmetric downward modulation case, for which no dynamics modulation was observed, there is no overlap. A circle is placed over this part of the figure for emphasis.

This suggests that modes beyond the two immediately involved in the dynamics modulation play a role in whether or not dynamics modulation occurs. Obviously this multimode effect would not be reflected in the two-mode model presented in Chapter V. For the downward modulation that does occur, the driving-force frequency must be on the outside of the target mode. That difference from the upward case tends to cause the downward case to, in straightforward manner, force a permanent downward mode transition (see Figures $4.27 \mathrm{~d}-\mathrm{f}$ and $4.28 \mathrm{~d}-\mathrm{f}$ ) under some circumstances and irregular modulation (see Figures $4.25 \mathrm{~b}-\mathrm{e}$ and $4.26 \mathrm{~b}-\mathrm{e}$ ) in others. 


\section{Chapter VII}

\section{Conclusions}

In this thesis, dynamics modulation was experimentally studied and simulated using a coupled oscillator van der Pol model. In dynamics modulation, two modes in a driven neon glow discharge alternate as the dominant mode as their response to the driving force alternates between spatiotemporal and temporal periodic pulling. This phenomenon was first noted by Koepke, Weltmann, and Selcher [Koepke et al. (1995)], who saw two limited but representative cases and proposed a mechanism [Weltmann et al. (2000)] by which it occurs. The intent of this dissertation, to document and test the mechanism, has been achieved.

Three sequences of time series data, as a function of increasing driving frequency, were presented. Two of these series were taken for one pair of mode frequencies on different days to show that the data is reproducible. The third was taken for the next higher pair of mode frequencies to show that the phenomenon is not limited to one pair of modes. Each of these data series shows the progression of the system from pure spatiotemporal behavior, through dynamics modulation, and ending at entrainment in the upper mode. Wave modes were examined by recording discharge luminosity time series using a photodiode. The system was periodically driven using a narrow-band ring dye laser tuned to resonance with the $588.35 \mathrm{~nm}$ metastable neon transition. The amplitude of the driving force was decreased (increased) by tuning the laser away from (nearer to) the center of the neon line, while the driving frequency was controlled by an acousto-optic modulator chopping the laser beam at the desired frequency.

Using a new extension of a mathematical treatment of periodic pulling presented in this thesis, the resulting experimental data were used to verify the predicted mechanism. This new extension 
included the definition of a reference beat frequency $\left(\Omega^{\prime}\right)$ which, along with the modified beat frequency $(\Omega)$ identified from the data for the spatiotemporal and temporal cases, allowed the entrainment parameter $(\alpha)$ and the normalized driving amplitude $(M)$ to be presented as a function of driving frequency for both portions of the dynamics modulation cycle. This was done here for the first time, and showed that the temporal entrainment parameter $\left(\alpha_{T}\right)$ goes to one as expected when the sequence of dynamics modulation ends in entrainment at the upper mode. The new extension also predicts an asymmetry between two modes pulling each other, depending on which one is pulling and which one is pulled, which is seen in the dynamics modulation data.

Arnol'd tongue boundaries identifying the edges of frequency entrainment regions in the driving amplitude-driving frequency plane were established for four different discharge currents. Differences were noted between modes that can occur naturally at a given discharge current, and thus appear as elongated on the driving amplitude axis, and those that occur only under driving, and are much shorter. Dynamics modulation was plotted in relation to the boundaries and used to verify the mechanism presented by Weltmann et al. (2000) in their dynamics phase diagram plot (Figure 2.10). These boundary plots are the first of their kind, showing Arnol'd tongue boundaries for optically-driven modes.

In addition to reproducing and studying the upward dynamics modulation, additional data were acquired for downward modulation, which had not been previously documented. This downward modulation exhibited qualitatively different behavior from the upward modulation described by Weltmann et al. (2000). Two representative cases were presented, and their characteristics described. The location of downward dynamics modulation on the Arnol'd tongue boundary plot was presented and shown not to be a mirror-symmetric version of the upward modulation case. The participation of multiple resonant modes in the dynamics modulation process was presented as a reason for this asymmetry.

Finally, a numerical model was presented that reproduced the signature of dynamics modulation. Two coupled van der Pol equations were chosen to model the mechanism described by Koepke, Weltmann, and Selcher, and the resulting time series was solved with a Runge-Kutta routine whose parameters could be adjusted as the simulation proceeded. The model successfully reproduced the qualitative behavior of dynamics modulation and reinforced the experimental verification of the proposed mechanism, but lacked sufficient complexity for a complete quantitative comparison. 
Appendices 
Page is intentionally blank 


\section{Appendix A.}

\section{Derivation of the phase evolution}

\section{equation}

In this appendix, the equation for phase evolution is derived. This derivation follows that of Lashinsky (1968), departing only to remove the assumption that $\omega_{i} \sim \omega_{0}$ that he made.

We start, as he did, with a general form of the van der Pol equation with a non-specific damping term:

$$
\frac{d^{2} x}{d t^{2}}-\epsilon f(x, \dot{x}) \frac{d x}{d t}+\omega_{0}^{2} x=\omega_{0}^{2} E \cos \omega_{i} t
$$

Now let the solution take the form

$$
x(t)=a(t) \sin \left[\omega_{i} t-\phi(t)\right] \equiv a(t) \sin \psi,
$$

which explicitly suggests that both the amplitude $(a)$ and phase $(\phi)$ are time dependent.

The time derivative of $\phi(t)$ is the piece that will allow a quantitative measure of periodic pulling. To get it, take time derivatives of $x(t)$ and put them into Eq. (A.1). We find that

$$
\frac{d x}{d t}=\frac{d a}{d t} \sin \psi+a\left(\omega_{i}-\frac{d \phi}{d t}\right) \cos \psi
$$

In computing the second derivative, we require that $a(t)$ and $\phi(t)$ be slowly varying, so that second 
order derivatives may be neglected. The result is

$$
\frac{d^{2} x}{d t^{2}} \cong 2 \omega_{i} \frac{d a}{d t} \cos \psi-\omega_{i}^{2} a \sin \psi+2 \omega_{i} a \frac{d \phi}{d t} \sin \psi
$$

Now, insert Eqs. (A.3) and (A.4) into Eq. (A.1):

$$
\begin{array}{r}
2 \omega_{i} \frac{d a}{d t} \cos \psi-\omega_{i}^{2} a \sin \psi+2 \omega_{i} a \frac{d \phi}{d t} \sin \psi \\
-\epsilon f(x, \dot{x})\left[\frac{d a}{d t} \sin \psi+\left(\omega_{i}-\frac{d \phi}{d t}\right) a \cos \psi\right]+\omega_{0}^{2} a \sin \psi=\omega_{0}^{2} E \cos \omega_{i} t .
\end{array}
$$

This can be simplified by expressing $\omega_{i} t$ in terms of $\psi$ and $\phi$ using Eq. (A.2) and by requiring $\epsilon$ to be small enough that terms like $\epsilon \frac{d a}{d t}$ and $\epsilon \frac{d \phi}{d t}$ can be neglected. The result is:

$$
\begin{array}{r}
2 \omega_{i} \frac{d a}{d t} \cos \psi-\omega_{i}^{2} a \sin \psi+2 \omega_{i} a \frac{d \phi}{d t} \sin \psi \\
-\epsilon f(x, \dot{x}) \omega_{i} a \cos \psi+\omega_{0}^{2} a \sin \psi=\omega_{0}^{2} E(\cos \psi \cos \phi-\sin \psi \sin \phi) .
\end{array}
$$

A set of coupled, first-order equations that describe the time variation of the amplitude and phase variation of the mode result if we set the coefficients of $\cos \psi$ and $\sin \psi$ to zero separately in Eq. (A.6):

$$
\begin{aligned}
\frac{d a}{d t} & =\frac{\epsilon}{2} f(x, \dot{x}) a+\frac{E}{2} \frac{\omega_{0}^{2}}{\omega_{i}} \cos \phi \quad \text { (general form) } \\
\frac{d \phi}{d t} & =\left(\frac{\omega_{i}^{2}-\omega_{0}^{2}}{2 \omega_{i}}\right)-\frac{E}{2 a} \frac{\omega_{0}^{2}}{\omega_{i}} \sin \phi \quad \text { (general form) }
\end{aligned}
$$

Lashinsky requires $\omega_{i} \sim \omega_{0}$, in which case

$$
\frac{\omega_{i}^{2}-\omega_{0}^{2}}{2 \omega_{i}} \sim\left(\omega_{i}-\omega_{0}\right) .
$$

This requirement simplifies the above equations to the form:

$$
\begin{aligned}
& \frac{d a}{d t}=\frac{\epsilon}{2} f(x, \dot{x}) a+\frac{E \omega_{0}}{2} \cos \phi \quad\left(\text { valid only if } \omega_{i} \sim \omega_{0}\right) \\
& \frac{d \phi}{d t}=\left(\omega_{i}-\omega_{0}\right)-\frac{E \omega_{0}}{2 a} \sin \phi \quad\left(\text { valid only if } \omega_{i} \sim \omega_{0}\right) .
\end{aligned}
$$




\section{Appendix B.}

\section{Computer code}

In this appendix, examples of MATLAB code used in Chapter V are reproduced.

\section{Main simulation routine}

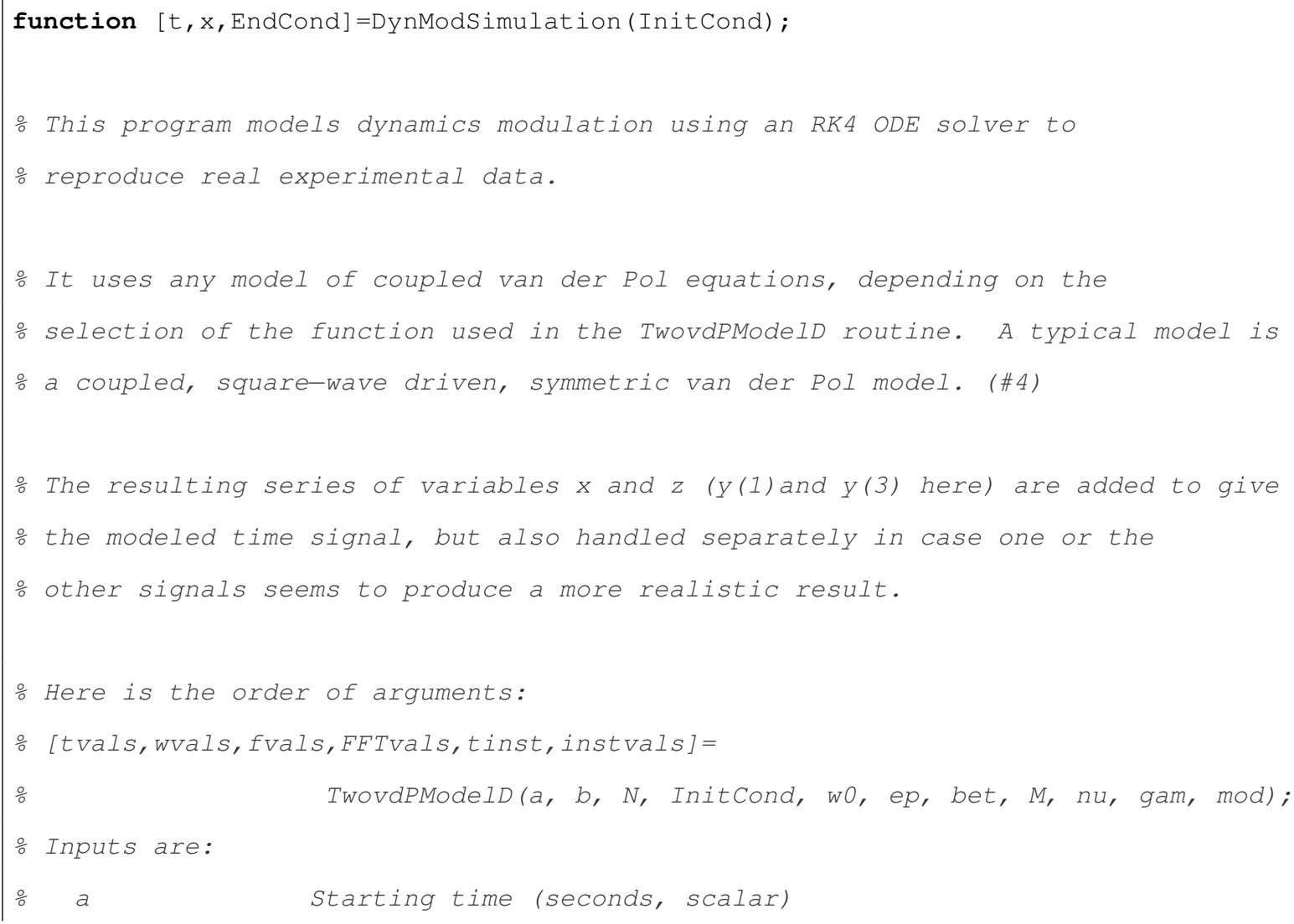




\section{SECTION 1. MAIN SIMULATION ROUTINE}

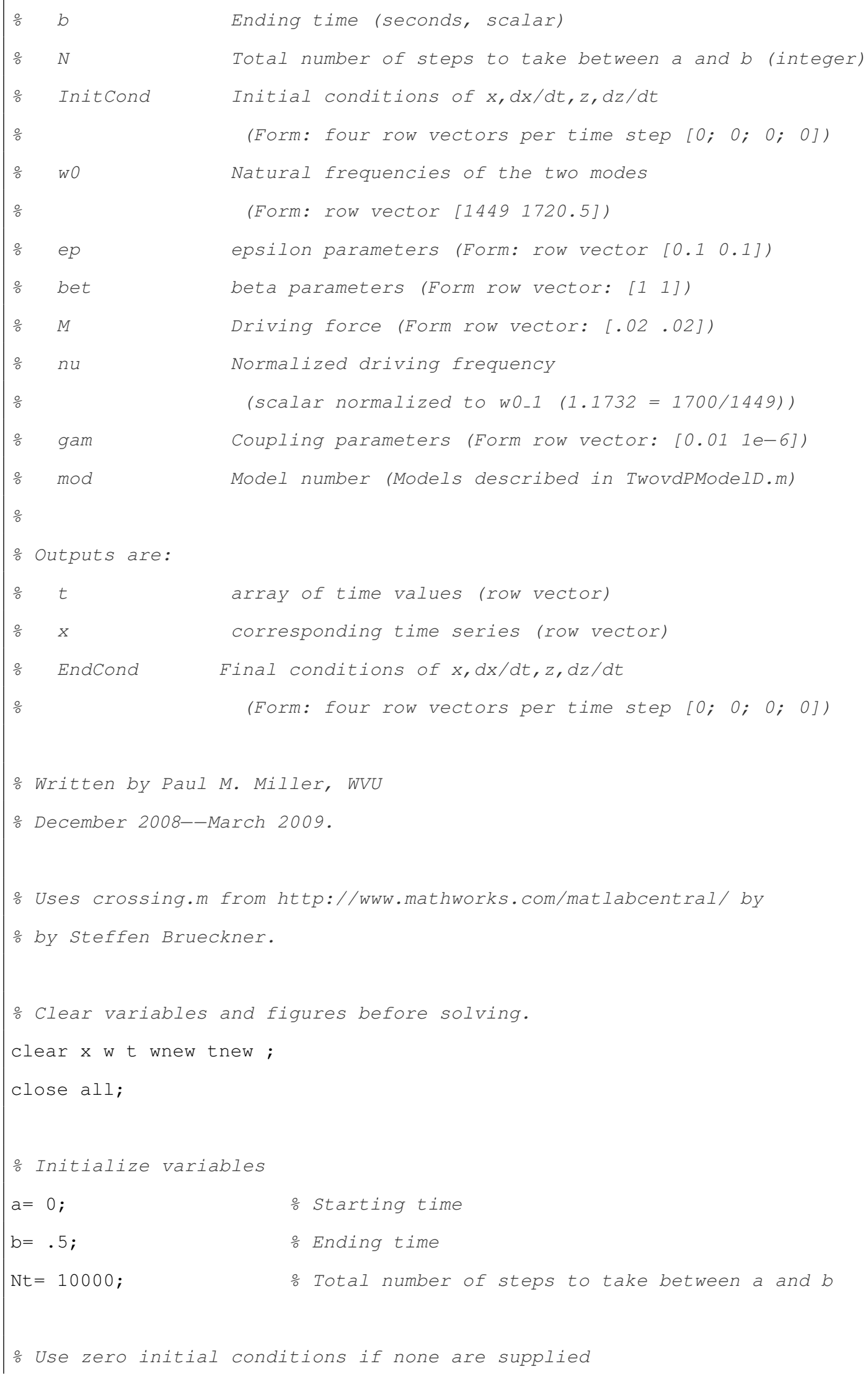


APPENDIX B.. COMPUTER CODE

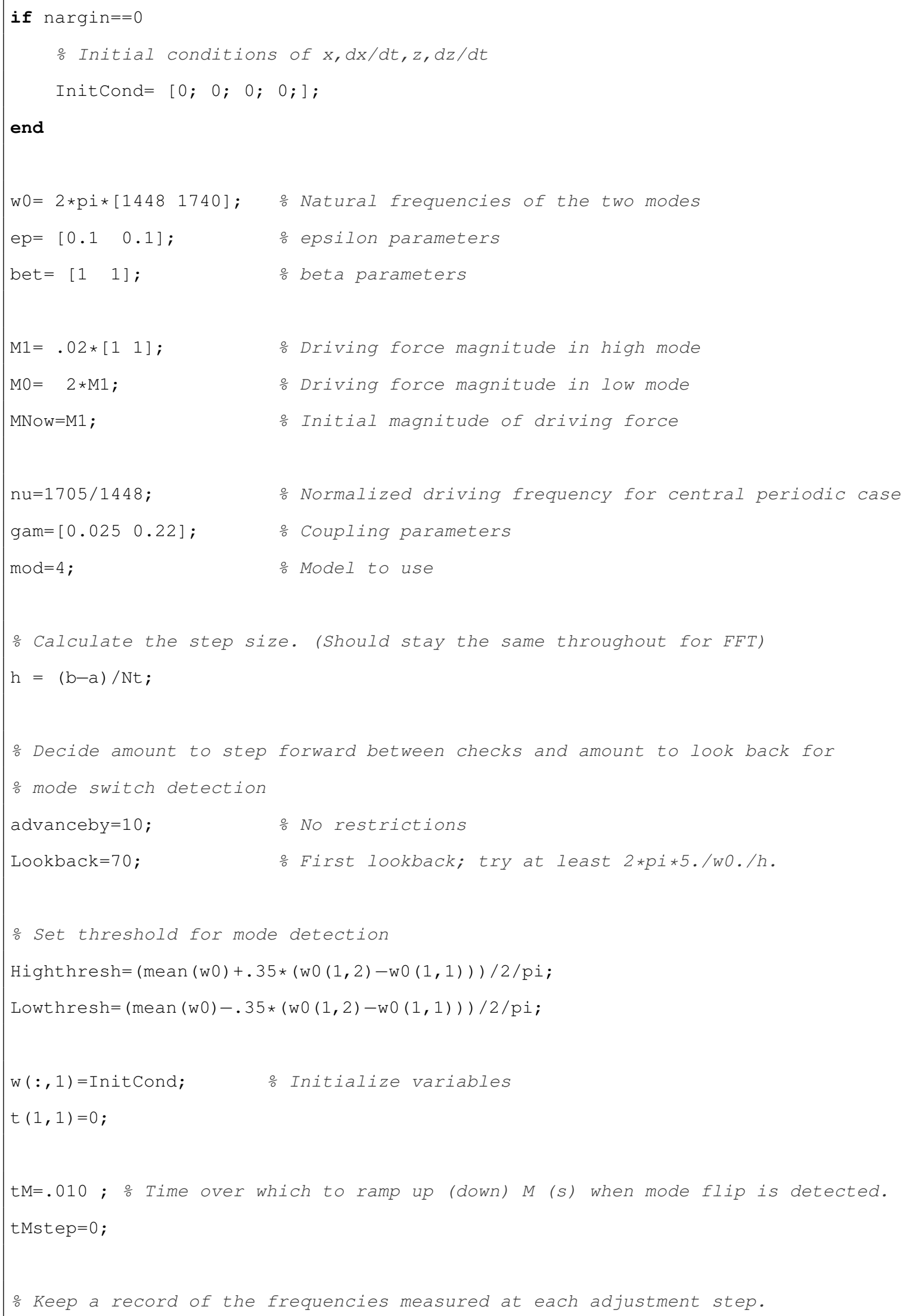




\section{SECTION 1. MAIN SIMULATION ROUTINE}

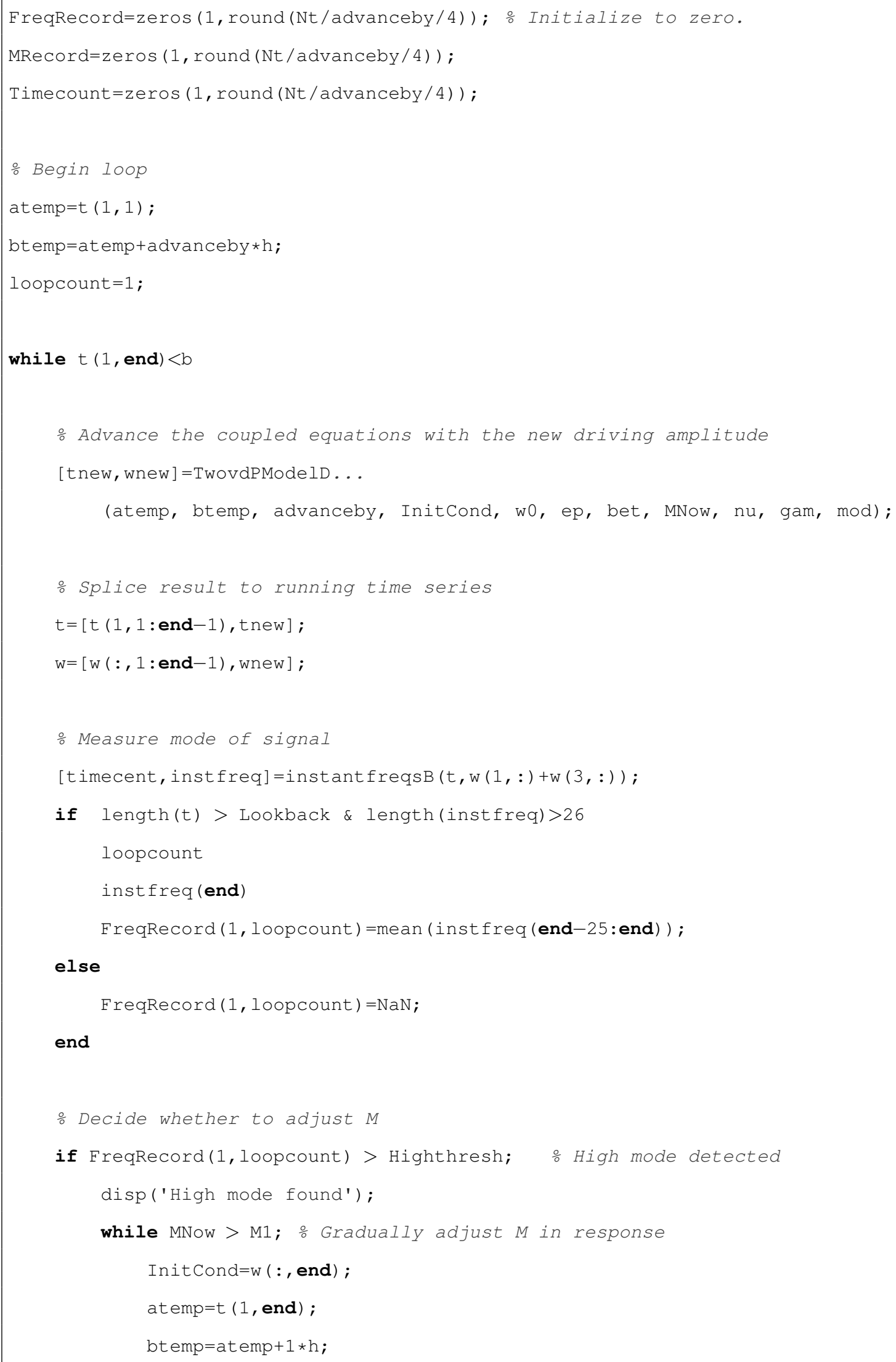




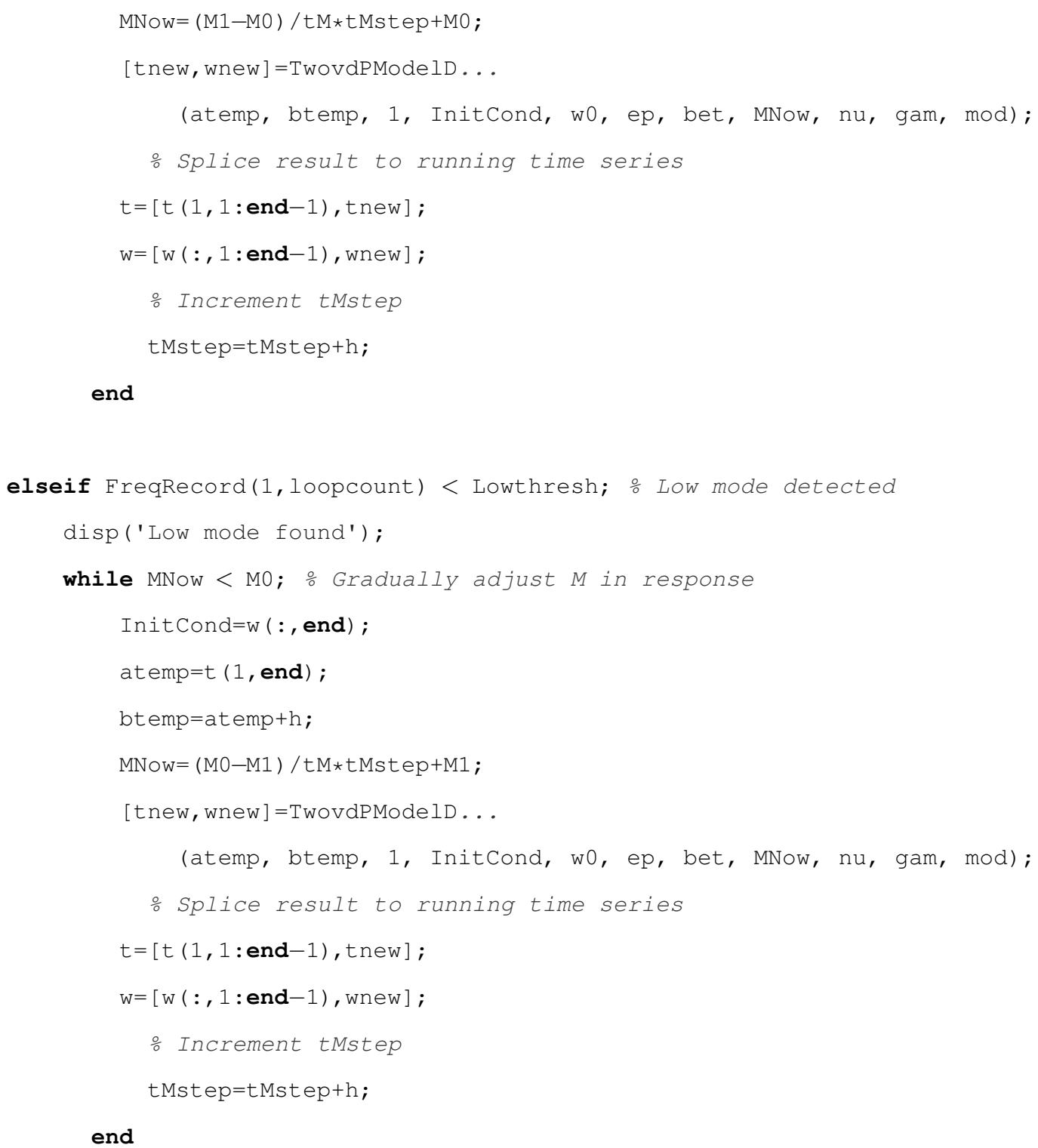




\section{SECTION 1. MAIN SIMULATION ROUTINE}

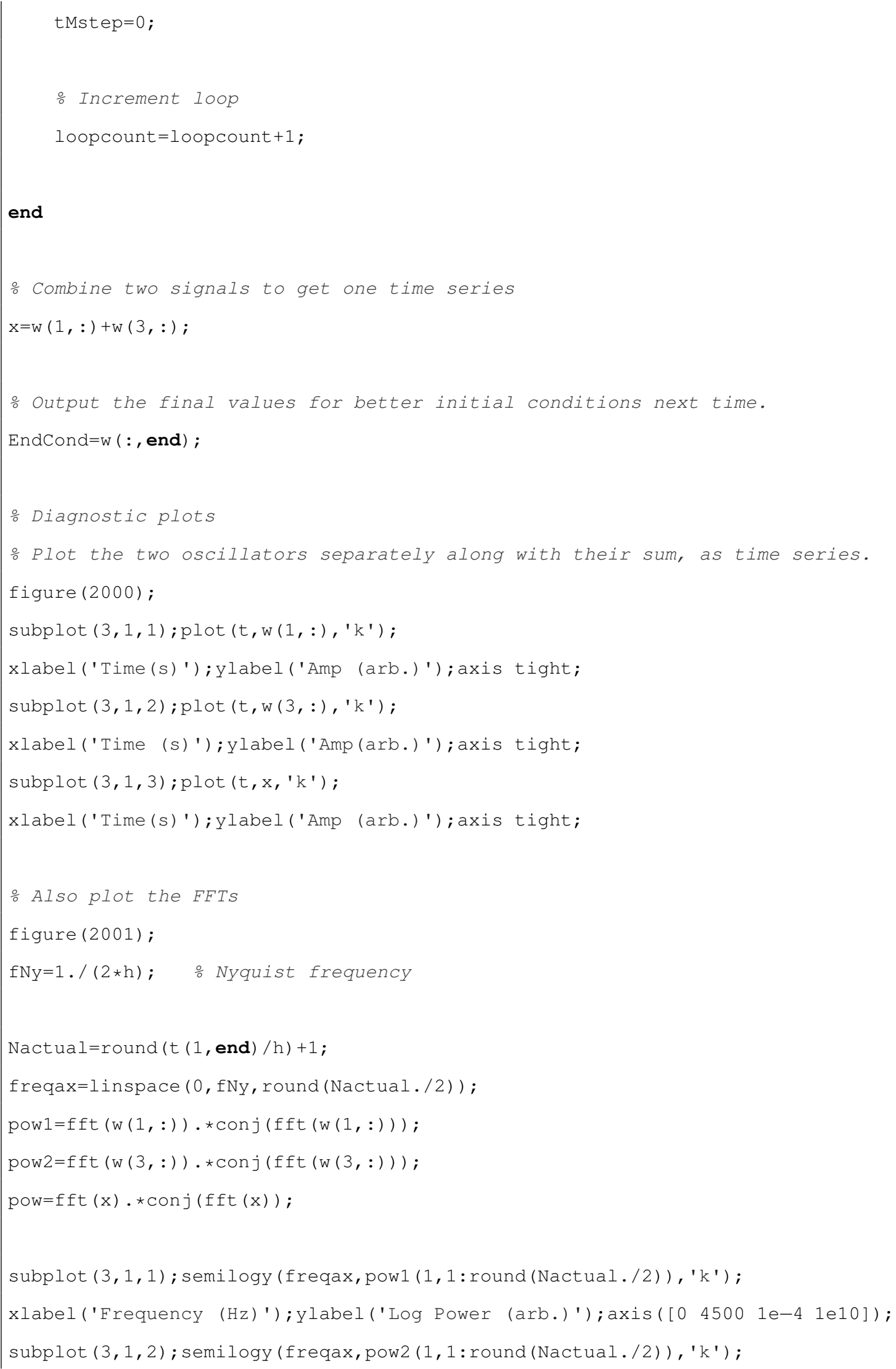


APPENDIX B.. COMPUTER CODE

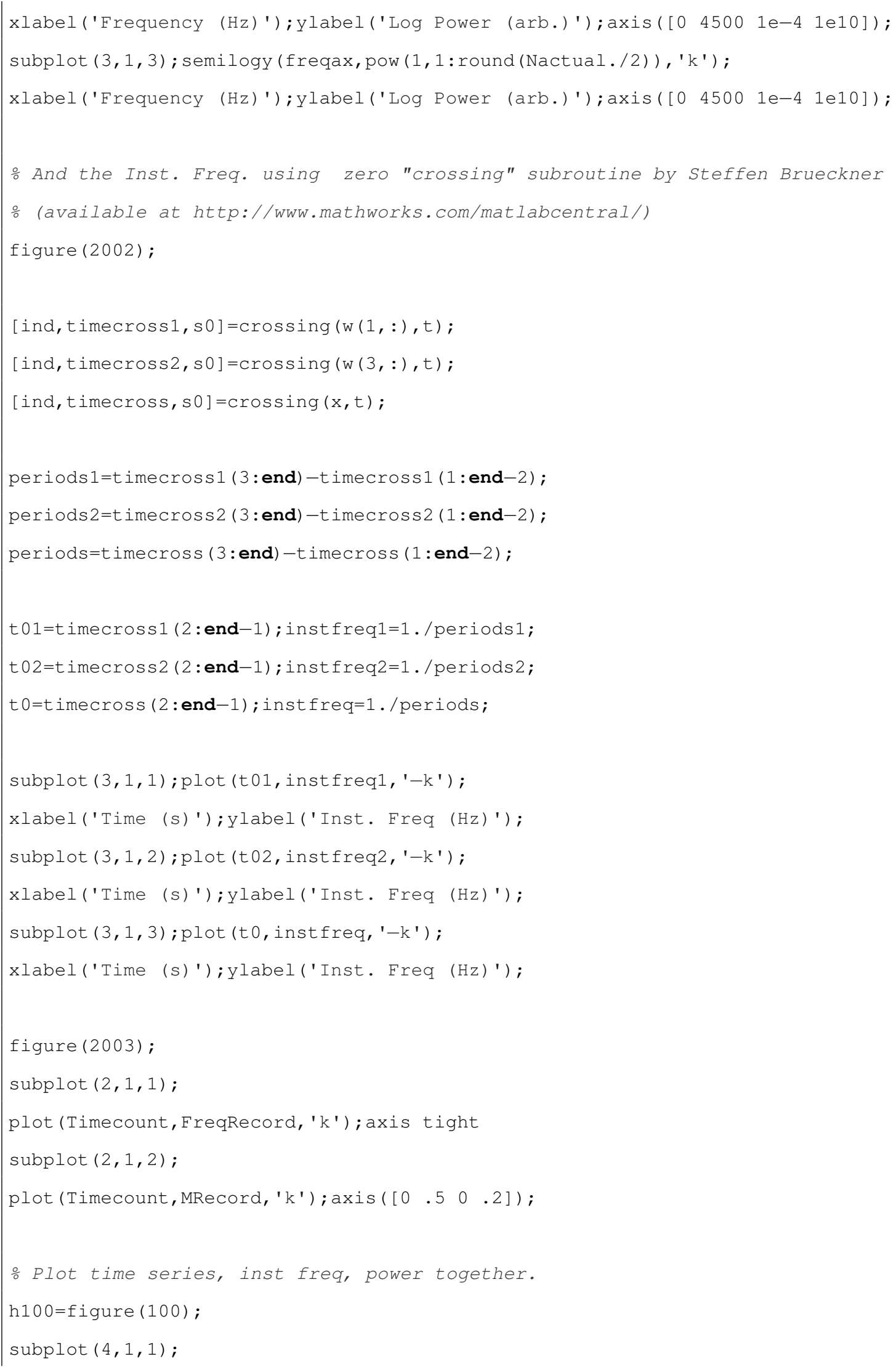


SECTION 2. RUNGE-KUTTA SUBROUTINE

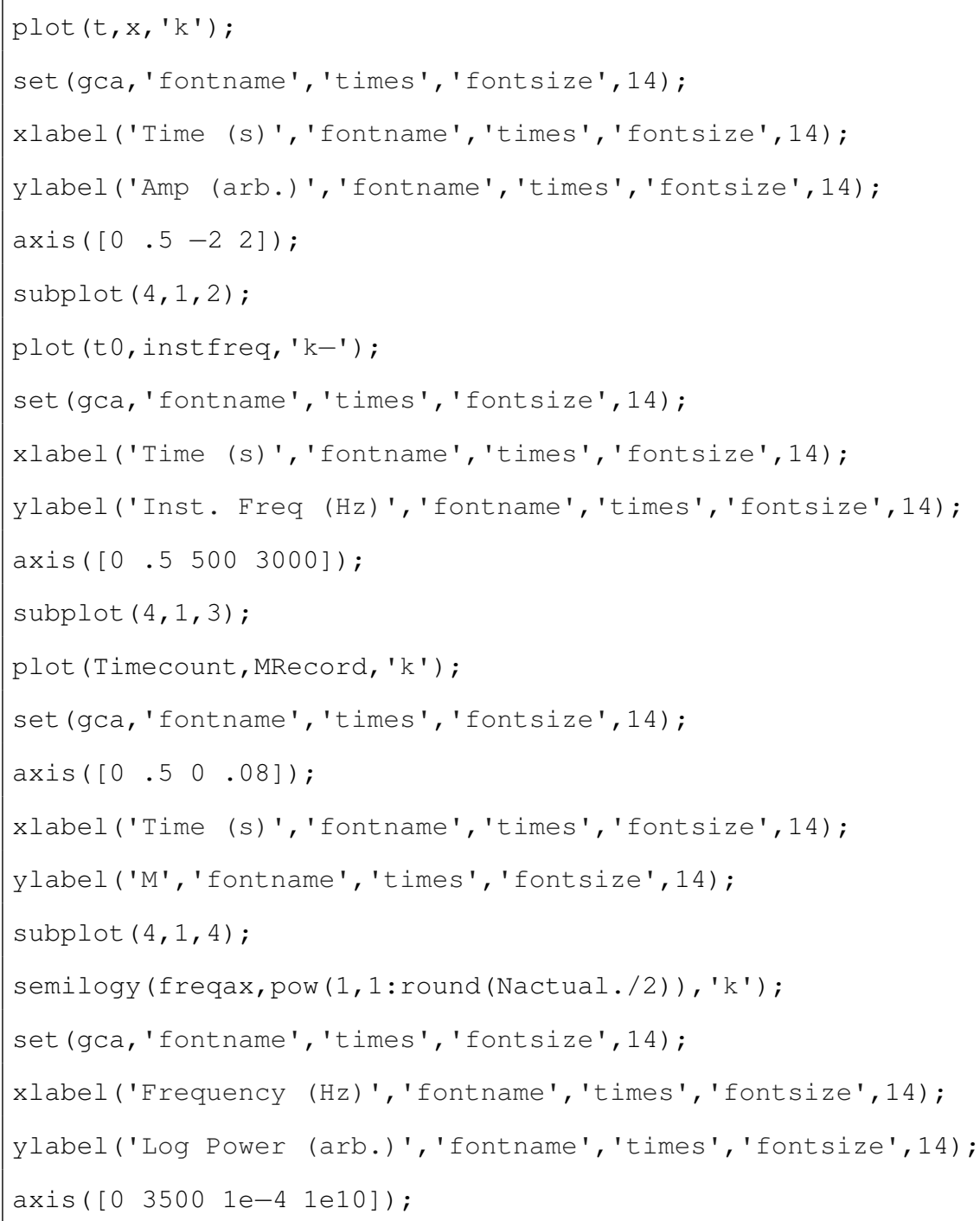

\section{Runge-Kutta subroutine}

function [tvals, wvals] =TwovdPModelD (a, b, N, InitCond, w0, ep, bet, M, nu, gam, mod) ;

․o This program models dynamics modulation using an RK4 ODE solver to

o reproduce real experimental data.

$\div$

응 allows the choice of the model of coupled van der Pol equations.

응 The result of variables $x$ and $z$ ( $y(1)$ and $y(3)$ here) representing the two

o oscillators are added to give 


\section{APPENDIX B.. COMPUTER CODE}

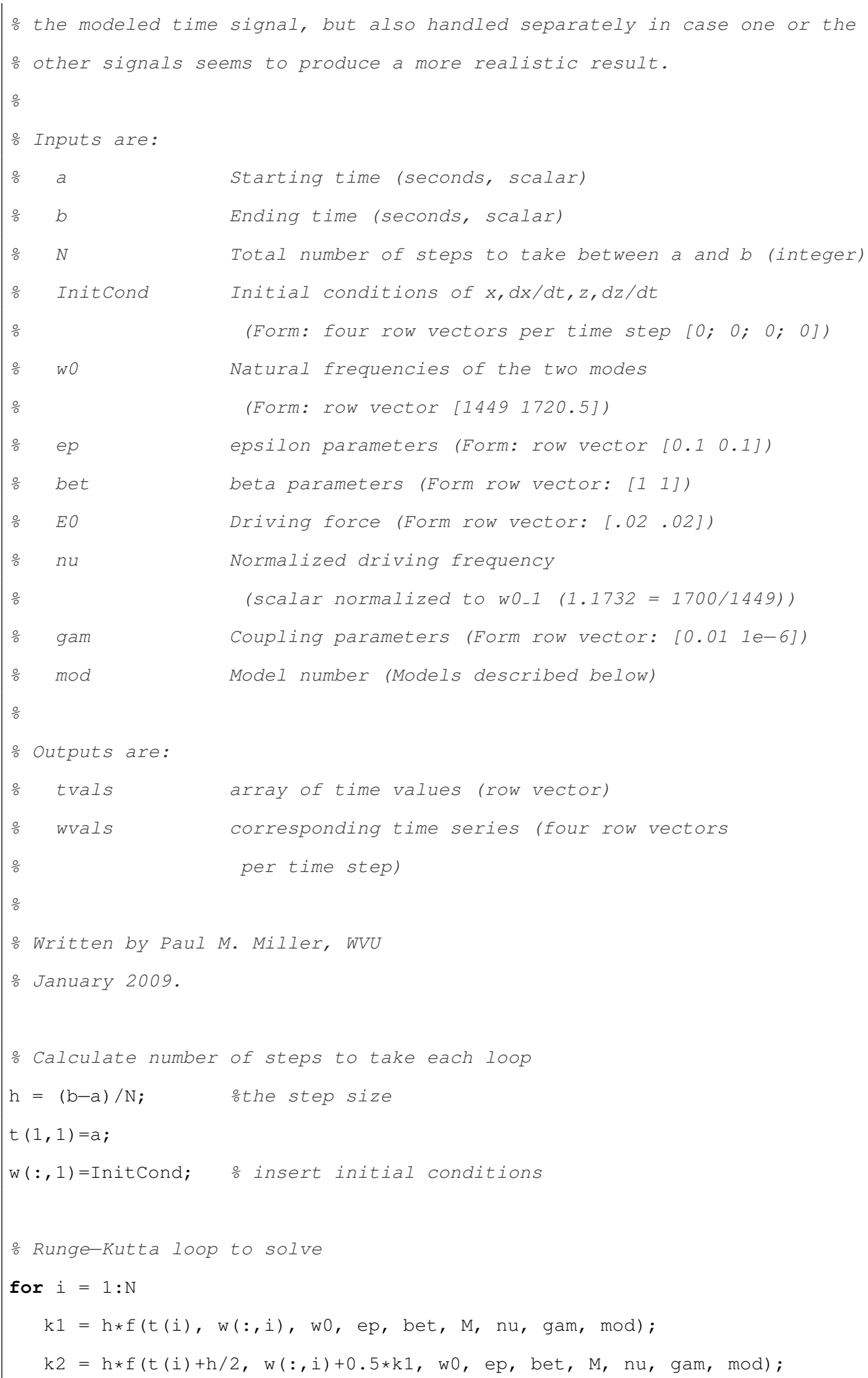




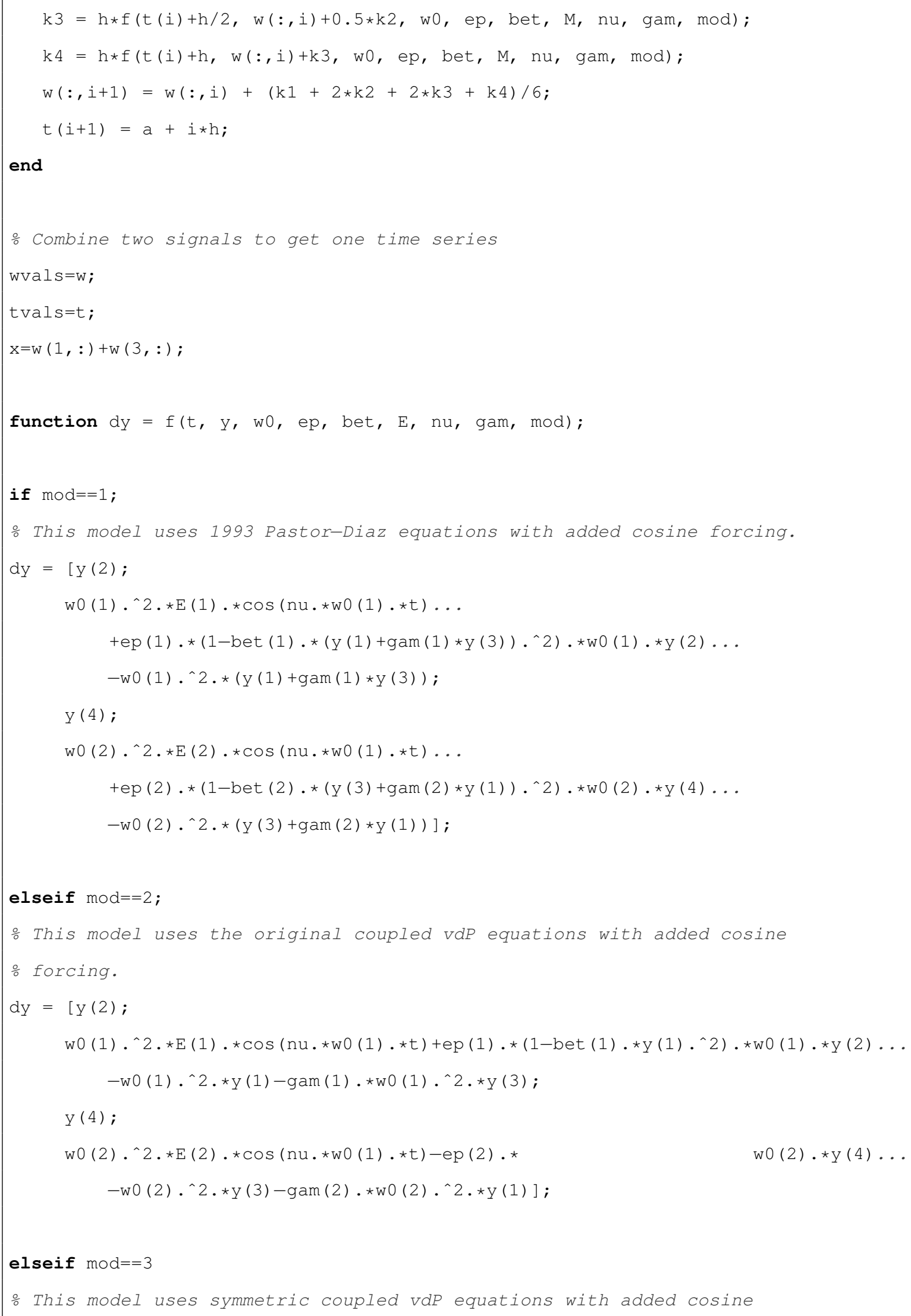




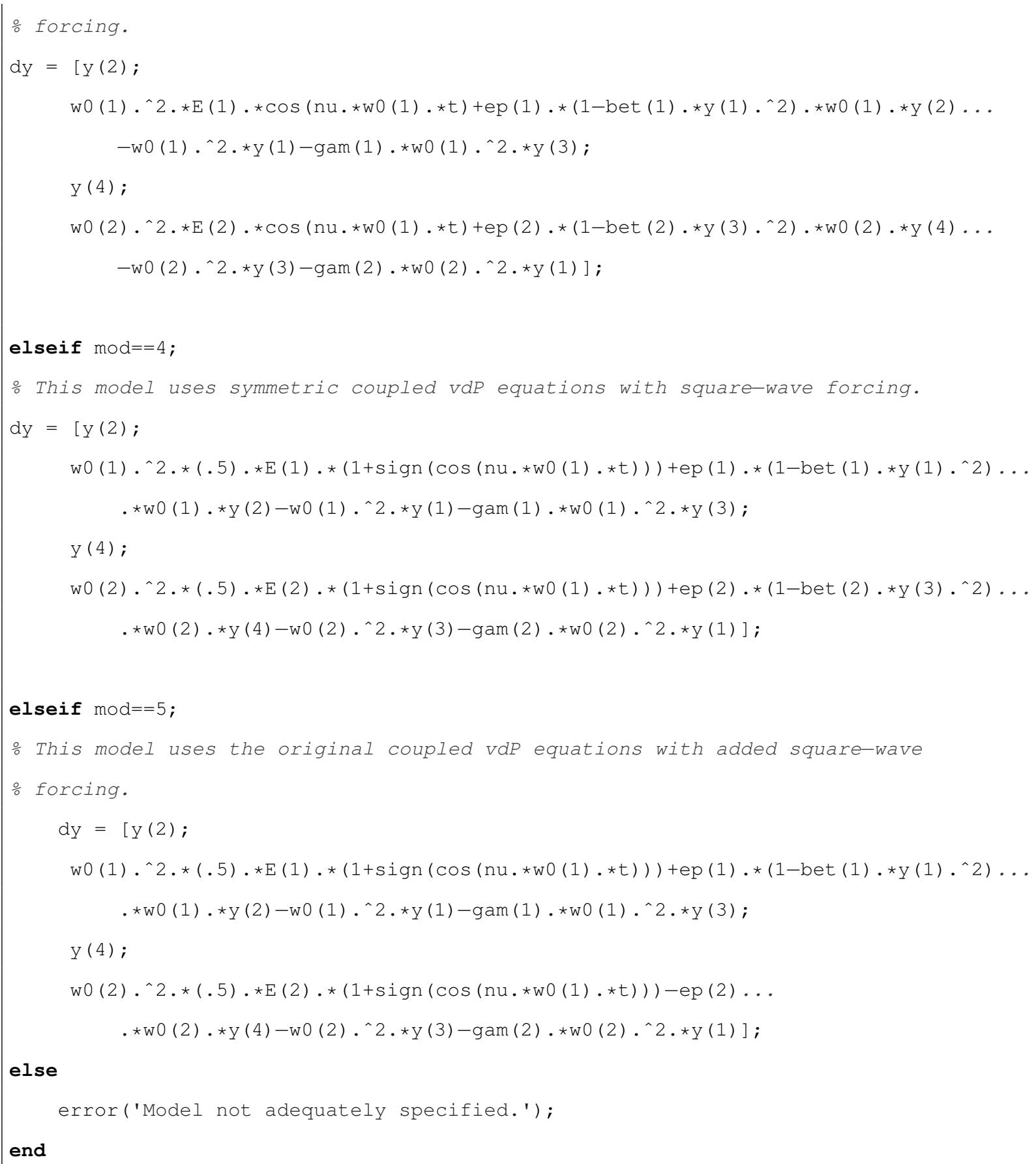


Page is intentionally blank 


\section{References}

Abria, M. (1848), Sur les lois de l'induction des courants par les courants, Ann. Chim., 7, 477.

Adler, R. (1946), A study of locking phenomena in oscillators, Proceedings of the IRE, 34(6), 351.

Armand, M. (1969), On the output spectrum of unlocked driven oscillators, Proceedings of the IEEE, 57, 798.

Arslanbekov, R., and V. Kolobov (2003), Two-dimensional simulations of the transition from Townsend to glow discharge and subnormal oscillations, J. Phys. D: Appl. Phys., 36(2), 2986.

Arslanbekov, R., and V. Kolobov (2005), 2-D simulations of striations in direct current glow discharges in argon, IEEE Transactions on Plasma Science, 33(2), 354.

Baierlein, R. (1983), Newtonian Dynamics, 1st ed., McGraw-Hill Companies, New York.

Block, D. (2001), Synchronization of drift waves and its effect on fluctuation-induced transport, Ph.D. thesis, University of Kiel, Kiel, Germany.

Boeuf, J. (2003), Plasma display panels: physics, recent developments and key issues, J. Phys. D: Appl. Phys., 36, R53.

Bogoliubov, N. N., and Y. A. Mitropolsky (1961), Asymptotic Methods in the Theory of Non-linear Oscillations, Gordon and Breach, New York.

Boyce, W. E., and R. C. DiPrima (1986), Elementary Differential Equations and Boundary Value Problems, fourth ed., John Wiley and Sons, New York.

Braun, T., J. Lisboa, R. Francke, and J. Gallas (1987), Observation of deterministic chaos in electrical discharges in gases, Phys. Rev. Lett., 59(6), 613. 


\section{REFERENCES}

Brown, A. C. (1995), An estimate of the largest Lyapunov exponent in a driven neon glow discharge plasma, Master's thesis, West Virginia University, Morgantown, WV.

Brown, S. C. (1978), A short history of gaseous electronics, in Gaseous Electronics: Electrical Discharges, vol. 1, edited by M. N. Hirsch and H. J. Oskam, chap. 1, pp. 1-18, Academic, New York.

Cheung, P., and A. Wong (1987), Chaotic behavior and period doubling in plasmas, Phys. Rev. Lett., $59(5), 551$.

Cheung, P., S. Donovan, and A. Wong (1988), Observations of intermittent chaos in plasmas, Phys. Rev. Lett., 61(12), 1360.

Cuccia, C. L. (1952), Harmonics, Sidebands, and Transients in Communication Engineering, McGraw-Hill, New York.

Dinklage, A., B. Bruhn, H. Deutsch, P. Jonas, and B.-P. Koch (1998), Observation of the spatiotemporal dynamics of ionization wave mode transitions, Phys. Plasmas, 5(4), 833.

Dunham, J., S. Bennett, and C. Butler (1991), Frequency entrainment in the relaxation oscillator method of optogalvanic spectroscopy, Inst. Phys. Conf. Ser., 113(6), 191.

Eckhaus, W. (1965), Studies in Nonlinear Stability Theory, Springer, New York.

Franklin, R. N. (1976), Plasma Phenomena in Gas Discharges, Clarendon Press, Oxford.

Garscadden, A. (1978), Ionization waves in glow discharges, in Gaseous Electronics: Electrical Discharges, vol. 1, edited by M. N. Hirsch and H. J. Oskam, chap. 2.2, pp. 65-107, Academic, New York.

Gerstenkorn, S., and P. Luc (1977), Atlas du spectre d'absorption de la molécule de'iode, vol. II, Laboratoire Aimé Cotton Laboratory, Orsay, France.

Gerstenkorn, S., and P. Luc (1985), Description of the absorption spectrum of iodine recorded by means of Fourier Transform Spectroscopy: the (B-X) system, J. Physique, 46, 867.

Gleick, J. (1987), Chaos: Making a New Science, Viking, New York. 


\section{REFERENCES}

Greiner, F., T. Klinger, and A. Piel (1995), Nonlinear dynamical behavior of thermionic low pressure discharges. I. simulation, Phys. Plasmas, 2(6), 1810.

Hirsch, M. N., and H. J. Oskam (Eds.) (1978), Gaseous Electronics: Electrical Discharges, vol. 1, Academic Press, New York.

Ingold, J. H. (1978), Anatomy of the discharge, in Gaseous Electronics: Electrical Discharges, vol. 1, edited by M. N. Hirsch and H. J. Oskam, chap. 2.1, pp. 19-64, Academic, New York.

Jonas, P., B. Bruhn, B.-P. Koch, and A. Dinklage (2000), Numerical investigations on strong pattern selecting Eckhaus instabilities in neon glow discharges, Phys. Plasmas, 7(2), 729.

Keesee, A. M. (2006), Neutral density profiles in argon helicon plasmas, Ph.D. thesis, West Virginia University, Morgantown, WV.

Klinger, T., F. Greiner, A. Rohde, A. Piel, and M. Koepke (1995), van der Pol behavior of relaxation oscillations in a periodically driven thermionic discharge, Phys. Rev. E, 52(4), 4316.

Koepke, M., and D. Hartley (1991), Experimental verification of periodic pulling in a nonlinear electronic oscillator, Phys. Rev. A, 44(10), 6877.

Koepke, M., T. Klinger, F. Seddighi, and A. Piel (1996), Periodic nonlinear wave-wave interaction in a plasma discharge with no external oscillatory driving force, Phys. Plasmas, 3(12), 4421.

Koepke, M., A. Dinklage, T. Klinger, and C. Wilke (2001), Spatiotemporal signatures of periodic pulling during ionization-wave-mode transitions, Phys. Plasmas, 8(4), 1432.

Koepke, M. E., K.-D. Weltmann, and C. A. Selcher (1995), Spatio-temporal laser perturbation of competing ionization waves in a neon glow discharge, Bull. Am. Phys. Soc., 40, 1716.

Kolobov, V. (2006), Striations in rare gas plasmas, J. Phys. D: Appl. Phys., 39(24), R487.

Lamb Jr, W. (1964), Theory of an optical maser, Phys. Rev, 134(6A), A1429.

Landa, P., N. Miskinova, and Y. Ponomarev (1980), lonization waves in low-temperature plasmas, Physics-Uspekhi, 23(12), 813. 


\section{REFERENCES}

Lashinsky, H. (1968), Periodic pulling and the transition to turbulence in a system with discrete modes, in Symposium on Turbulence of Fluids and Plasmas, edited by J. Fox, pp. 29-46, Polytechnic Institute of Brooklyn, Polytechnic Press, New York.

Lashinsky, H. (1969), Mathematical models for nonlinear mode interactions in bounded plasmas, in Nonlinear Effects in Plasmas, edited by G. Kalman and M. Feix, p. 451, Second Orsay Summer Institute, Gordon and Breach, New York.

Lorenz, E. N. (1963), Deterministic nonperiodic flow, J. Atmos. Sci., 20, 130.

Mallat, S. (1999), A Wavelet Tour of Signal Processing, 2nd ed. ed., Academic Press, London.

Meggers, W. F., and C. J. Humpreys (1934), Bur. Stand. J. Res., 13, 293.

Minorsky, N. (1962), Nonlinear Oscillations, Robert E. Krieger Publishing Company, Malabar, Florida.

Moore, C. E. (1971), Atomic Energy Levels, vol. I, U.S. Government Printing Office, Washington, DC.

Novák, M. (1960), Spatial period of moving striations as function of electric field strength in glow discharge, Czech. J. Phys., Sect. B, 10, 954.

Oleson, N. L., and A. W. Cooper (1968), Moving striations, Adv. Electron. Electron Phys., 24, 155.

Ott, E., C. Grebogi, and J. Yorke (1990), Controlling chaos, Phys. Rev. Lett., 64(11), 1196.

Pastor, I., V. Pérez-García, F. Encinas, and J. Guerra (1993), Ordered and chaotic behavior of two coupled van der pol oscillators, Phys. Rev. E, 48(1), 171.

Pastor-Díaz, I., and A. López-Fraguas (1995), Dynamics of two coupled van der pol oscillators, Phys. Rev. E, 52(2), 1480.

Pekárek, L. (1968), Ionization waves (striations) in a discharge plasma, Physics-Uspekhi, 11(2), 188.

Pekárek, L., K. Mašek, and K. Rohlena (1970), A theory of ion-guided and metastable-guided varieties of ionization waves (striations), Czech. J. of Phys., 20(8), 879. 


\section{REFERENCES}

Pfau, S., A. Rutscher, and K. Wojaczek (1969), Beitr. Plasmaphys., 9, 333.

Pupp, W. (1932), Z. Physik, 33, 844.

Ralchenko, Y., A. E. Kramida, J. Reader, and NIST ASD Team (2008), NIST Atomic Spectra Database (version 3.1.5), [Online]. Available: http://physics.nist.gov/asd3, National Institute of Standards and Technology, Gaithersburg, MD.

Rayment, S. (1974), The role of the electron energy distribution in ionization waves, J. Phys. D: Appl. Phys., 7, 871.

Scime, E., R. Hardin, C. Biloiu, A. M. Keesee, and X. Sun (2007), Flow, flow shear, and related profiles in helicon plasmas, Phys. Plasmas, 14(4), 043,505.

Sheridan, T. E., M. E. Koepke, C. A. Selcher, and T. N. Good (1993), Periodic pulling in a driven relaxation oscillator, Proc. SPIE, 2039, 158.

Strogatz, S. H. (1994), Nonlinear Dynamics and Chaos, Westview Press, Cambridge, MA.

Ströhlein, G., and A. Piel (1989), Experimental evidence of a low-dimensional attractor in the coupling of drift- and ion-sound waves, Phys. Fluids B, 1, 1168.

Tsuru, T. (1976), Nonlinear resonance phenomena of electron plasma oscillations by beam modulation, J. Phys. Soc. Jpn., 40(2), 548.

van der Pol, B. (1934), The nonlinear theory of electric oscillations, Proceedings of the IRE, 22(9), 1051.

van der Pol, B., and J. van der Mark (1927), Frequency demultiplication, Nature, 120(3019), 363.

Weixing, D., H. Wei, W. Xiaodong, and C. Yu (1993), Quasiperiodic transition to chaos in a plasma, Phys. Rev. Lett., 70(2), 170.

Weltmann, K.-D., T. Bräuer, H. Deutsch, and C. Wilke (1995a), Investigations about the influence of the external circuit elements on the propagation of ionization waves in the positive column of a neon glow discharge, Contrib. Plasma Phys., 35(3), 225. 
Weltmann, K.-D., T. Klinger, and C. Wilke (1995b), Experimental control of chaos in a periodically driven glow discharge, Phys. Rev. E, 52(2), 2106.

Weltmann, K.-D., M. Koepke, and C. Selcher (2000), Spatiotemporal laser perturbation of competing ionization waves in a neon glow discharge, Phys. Rev. E, 62(2), 2773.

Wiese, W. L., M. W. Smith, and B. M. Glennon (Eds.) (1966), Atomic Transition Probabilities: Hydrogen Through Neon, vol. I, U.S. Government Printing Office, Washington, DC.

Yan, G. Y., K. I. Fujii, and A. L. Schawlow (1990), Relaxation-oscillator detection of optogalvanic spectra, Optics Letters, 15(2), 142. 


\section{Tables}


Page is intentionally blank 


\begin{tabular}{|cccc|}
\hline \hline $\begin{array}{c}\text { Ionization } \\
\text { Wave Type }\end{array}$ & $\begin{array}{c}\text { Relative } \\
\text { Speed }\end{array}$ & $\begin{array}{c}E \lambda(\text { Novák) } \\
(\mathrm{V})\end{array}$ & $\begin{array}{c}E \lambda(2006) \\
(\mathrm{V})\end{array}$ \\
\hline$p$ & slow & 9.2 & 9.8 \\
$s^{\prime}$ & slow & 19.5 & 20 \\
\hline$r$ & fast & 12.7 & 13.5 \\
$s$ & fast & 19.5 & 20 \\
\hline
\end{tabular}

Table 1.1: The Novák constants for ionization waves in low temperature plasmas. The first list comes from Novák (1960) and reflects the original empirical values. The second list comes from a recent review [Kolobov (2006)], and includes theoretical considerations.

\begin{tabular}{|rrrrc|}
\hline \hline Mode & $\begin{array}{r}\text { Low Frequency } \\
(\mathrm{Hz})\end{array}$ & $\begin{array}{r}\text { High Frequency } \\
(\mathrm{Hz})\end{array}$ & $\begin{array}{r}\text { Range } \\
(\mathrm{Hz})\end{array}$ & $\begin{array}{r}\text { Separation } \\
(\mathrm{Hz})\end{array}$ \\
\hline$f 1$ & 590 & 602 & 12 & N/A \\
$f 2$ & 649 & 691 & 42 & 59 \\
$f 3$ & 724 & 795 & 71 & 75 \\
$f 4$ & 845 & 912 & 67 & 121 \\
$f 5$ & 1005 & 1043 & 38 & 160 \\
$f 6$ & 1202 & 1242 & 40 & 197 \\
$f 7$ & 1436 & 1471 & 35 & 234 \\
$f 8$ & 1698 & 1742 & 44 & 262 \\
$f 9$ & 1980 & 2029 & 49 & 282 \\
\hline
\end{tabular}

Table 4.1: Approximate frequency ranges of the glow discharge modes. Frequency range is the difference between the high and low extremes of each mode. Mode frequency separation is measured from the low extreme of each mode to the low extreme of the mode below.

\begin{tabular}{|rrrcc|}
\hline \hline $\begin{array}{r}\text { Data } \\
\text { Set }\end{array}$ & $\begin{array}{r}\text { Modes } \\
\text { Involved }\end{array}$ & $\begin{array}{r}\text { \# of } \\
\text { Files }\end{array}$ & $\begin{array}{c}\text { \# of Driving } \\
\text { Frequencies Used }\end{array}$ & $\begin{array}{c}\text { Laser } \\
\text { Wavelength }(\mathrm{nm})\end{array}$ \\
\hline 1 & $f 7$ to $f 8$ & 68 & 12 & 588.351268 \\
2 & $f 7$ to $f 8$ & 53 & 11 & 588.351320 \\
3 & $f 8$ to $f 9$ & 44 & 11 & 588.351147 \\
4 & $f 8$ to $f 7$ & 52 & 12 & 588.351247 \\
5 & $f 8$ to $f 7$ & 96 & 19 & 588.351547 \\
\hline
\end{tabular}

Table 4.2: Summary of the main dynamics modulation time series data sets reported in Chapter IV. 


\begin{tabular}{|ccrcc|}
\hline \hline $\begin{array}{c}\text { Data } \\
\text { Set }\end{array}$ & $\begin{array}{c}f_{i} \\
(\mathrm{~Hz})\end{array}$ & $\begin{array}{r}\text { Offset from entrainment } \\
(\mathrm{Hz})\end{array}$ & $\begin{array}{r}\text { Average } \Omega_{T} / 2 \pi \\
(\mathrm{Hz})\end{array}$ & $\begin{array}{c}f_{D M} \\
(\mathrm{~Hz})\end{array}$ \\
\hline 1 & 1693 & -19 & $22.3 \pm 0.5$ & $22.2 \pm 0.1$ \\
1 & 1696 & -16 & $22.0 \pm 0.4$ & $21.4 \pm 0.1$ \\
1 & 1700 & -12 & $20.2 \pm 0.4$ & $20.2 \pm 0.1$ \\
1 & 1704 & -8 & $17.5 \pm 0.6$ & $17.5 \pm 0.1$ \\
1 & 1708 & -4 & $12.5 \pm 0.5$ & $12.7 \pm 0.1$ \\
\hline 2 & 1690 & -23 & $25.6 \pm 0.6$ & $25.7 \pm 0.1$ \\
2 & 1695 & -18 & $24.0 \pm 0.4$ & $24.1 \pm 0.1$ \\
2 & 1700 & -13 & $21.0 \pm 0.4$ & $21.2 \pm 0.1$ \\
2 & 1705 & -8 & $17.0 \pm 0.4$ & $16.5 \pm 0.1$ \\
\hline 3 & 1985 & -28 & $30.4 \pm 1.5$ & $30.5 \pm 0.1$ \\
3 & 1990 & -23 & $30.3 \pm 0.4$ & $29.3 \pm 0.1$ \\
3 & 1995 & -18 & $28.0 \pm 0.4$ & $28.2 \pm 0.1$ \\
3 & 2000 & -13 & $25.0 \pm 0.4$ & $25.0 \pm 0.1$ \\
\hline
\end{tabular}

Table 6.1: Comparison of temporal beat frequency $\Omega_{T} / 2 \pi$, taken from the power spectrum, to the direct measurement of periodic dynamics modulation frequency $f_{D M}$ taken from periods on instantaneous frequency series. Agreement is excellent. 


\section{Figures}




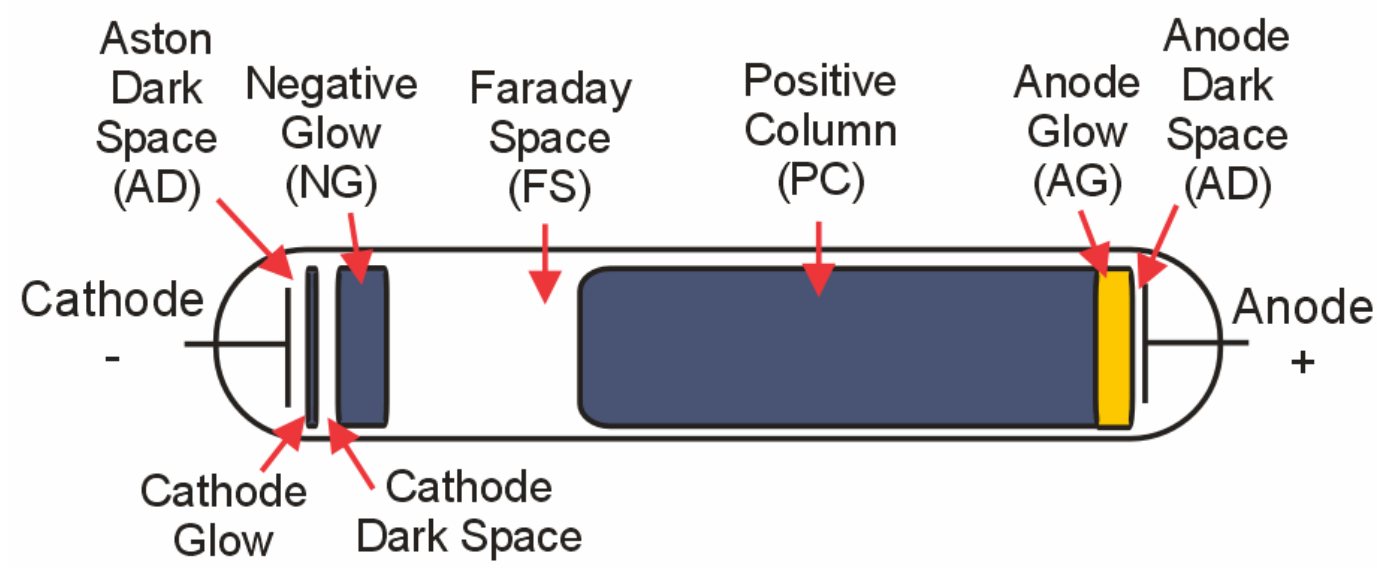

Figure 1.1: A schematic diagram of the named parts of a glow discharge column. Depending on the experimental parameters of the discharge tube and its circuit elements, some of the parts may not be visible and their relative sizes may vary. [Figure created by Ian Tresman. Used under Creative Commons Attribution 2.5.] 


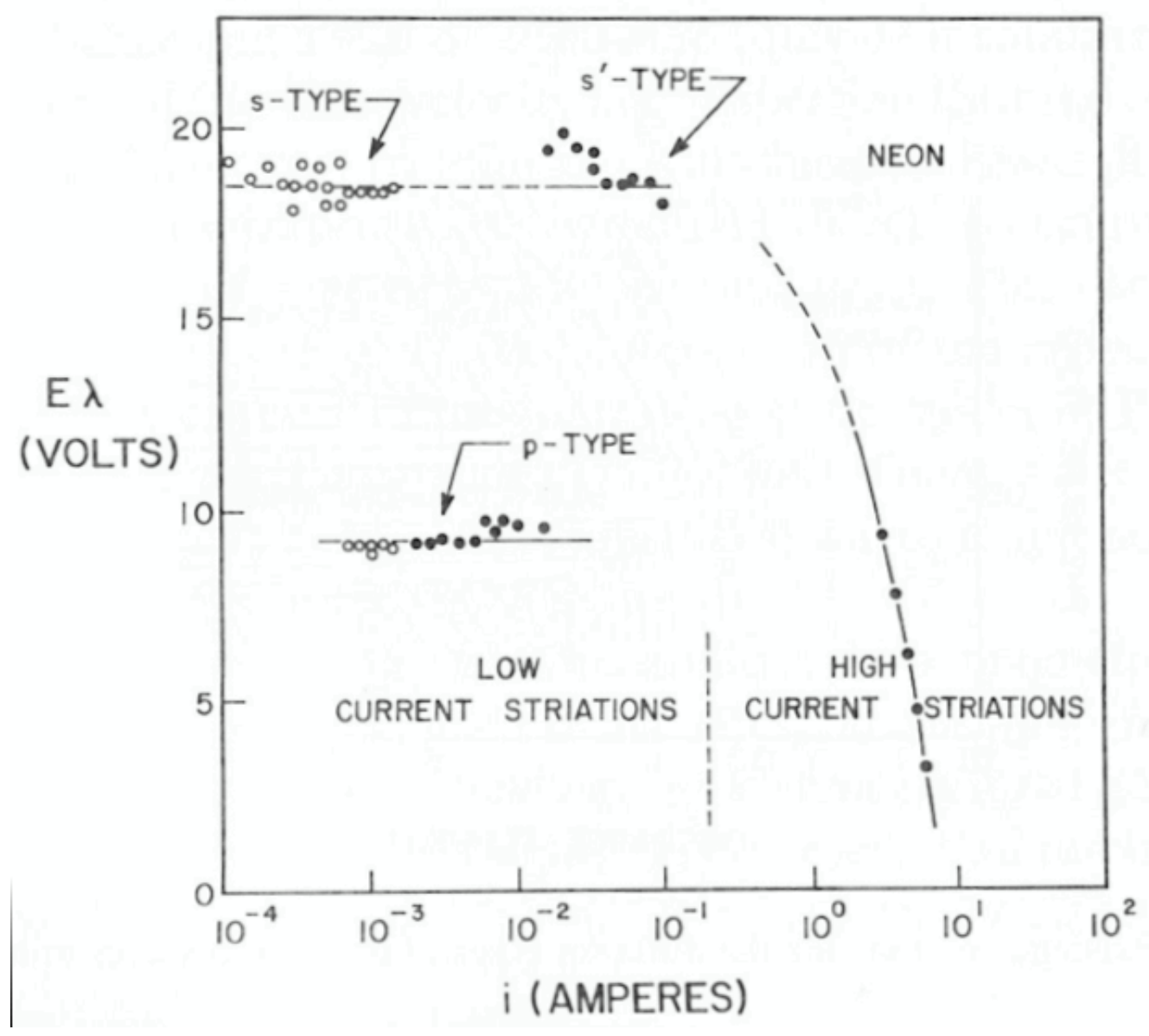

Figure 1.2: $E \lambda$ versus discharge current. Different ionization wave types can be unambiguously distinguished using their Novák constants. Note that all four types do not occur at all pressures, which is why no $r$ waves appear in this plot, and that striations at higher currents do not follow the Novák rule. [From Garscadden (1978).] 


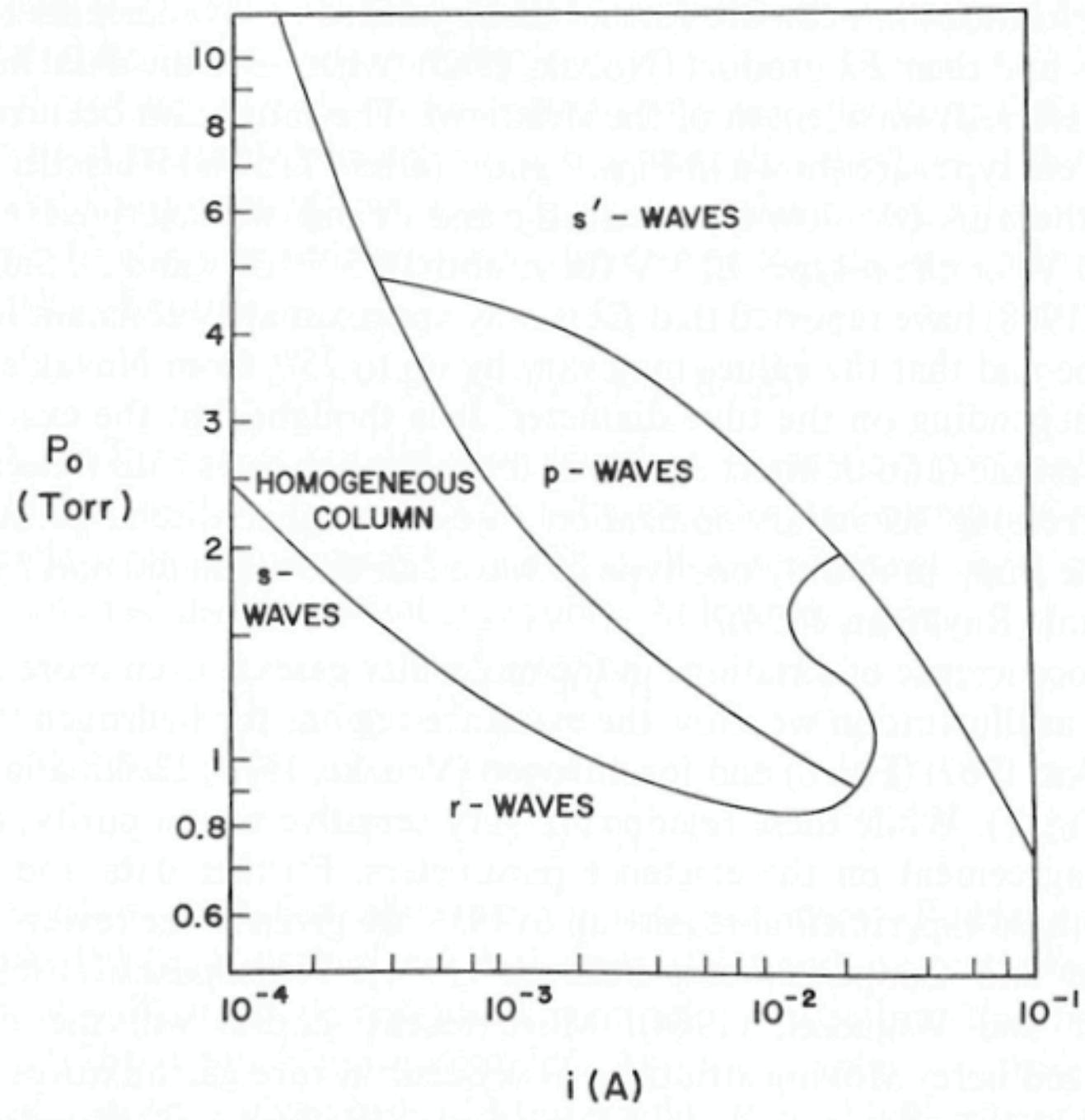

Figure 1.3: Ionization wave map. The regions in which the four kinds of ionization waves exist can be mapped against discharge current and pressure. Note the homogenous column in which no ionization waves occur. [From Garscadden (1978).] 


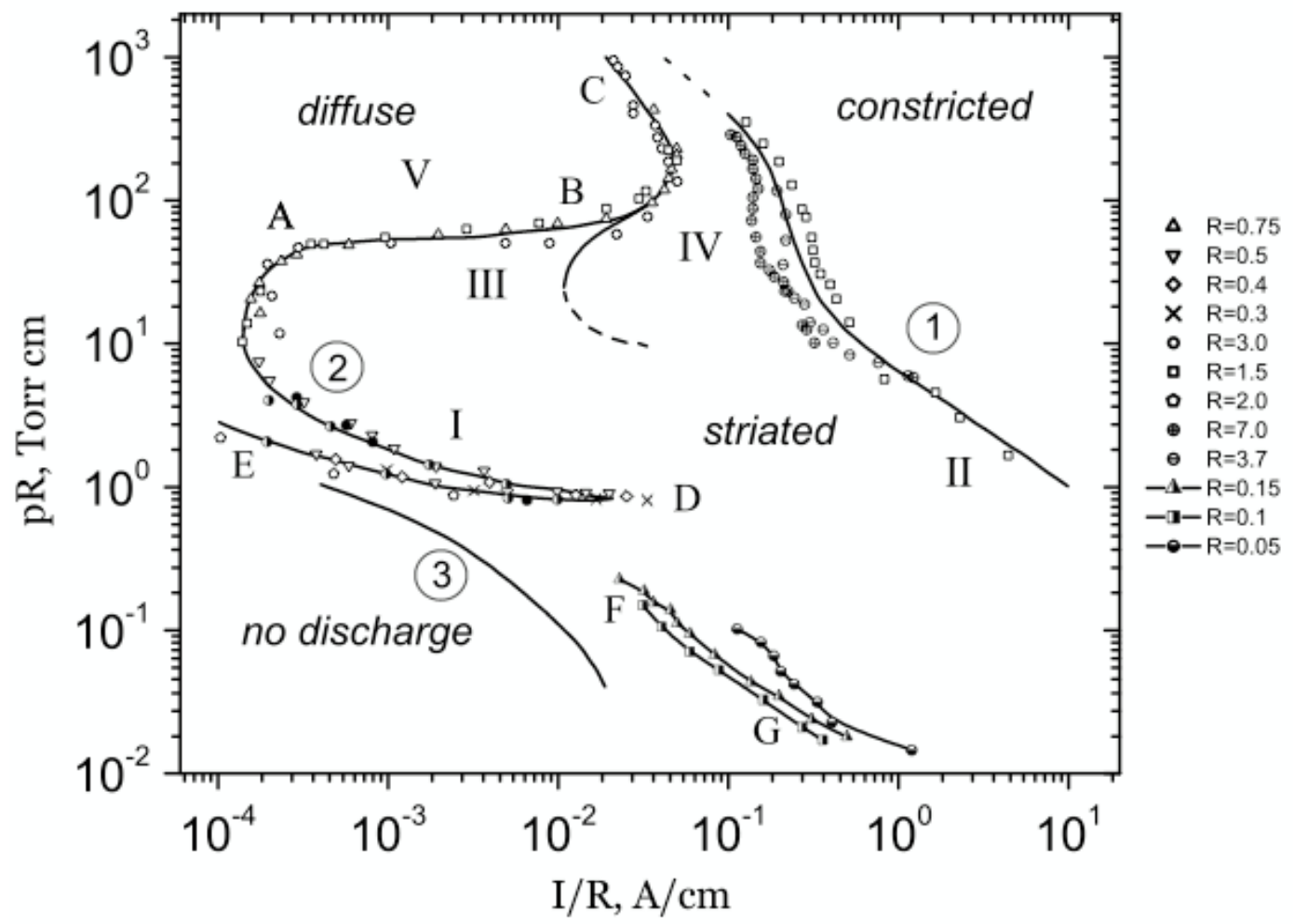

Figure 1.4: Possible wave states in the positive column of a neon discharge tube. Note the region labeled striated. Work in this thesis takes place at $p R \sim 1.5$ Torr $\mathrm{cm}$ and $I / r \sim 10^{-2} \mathrm{~A} / \mathrm{cm}$, near the I on the figure. The Pupp limit is indicated by curve labeled 1. [From Kolobov (2006).] 


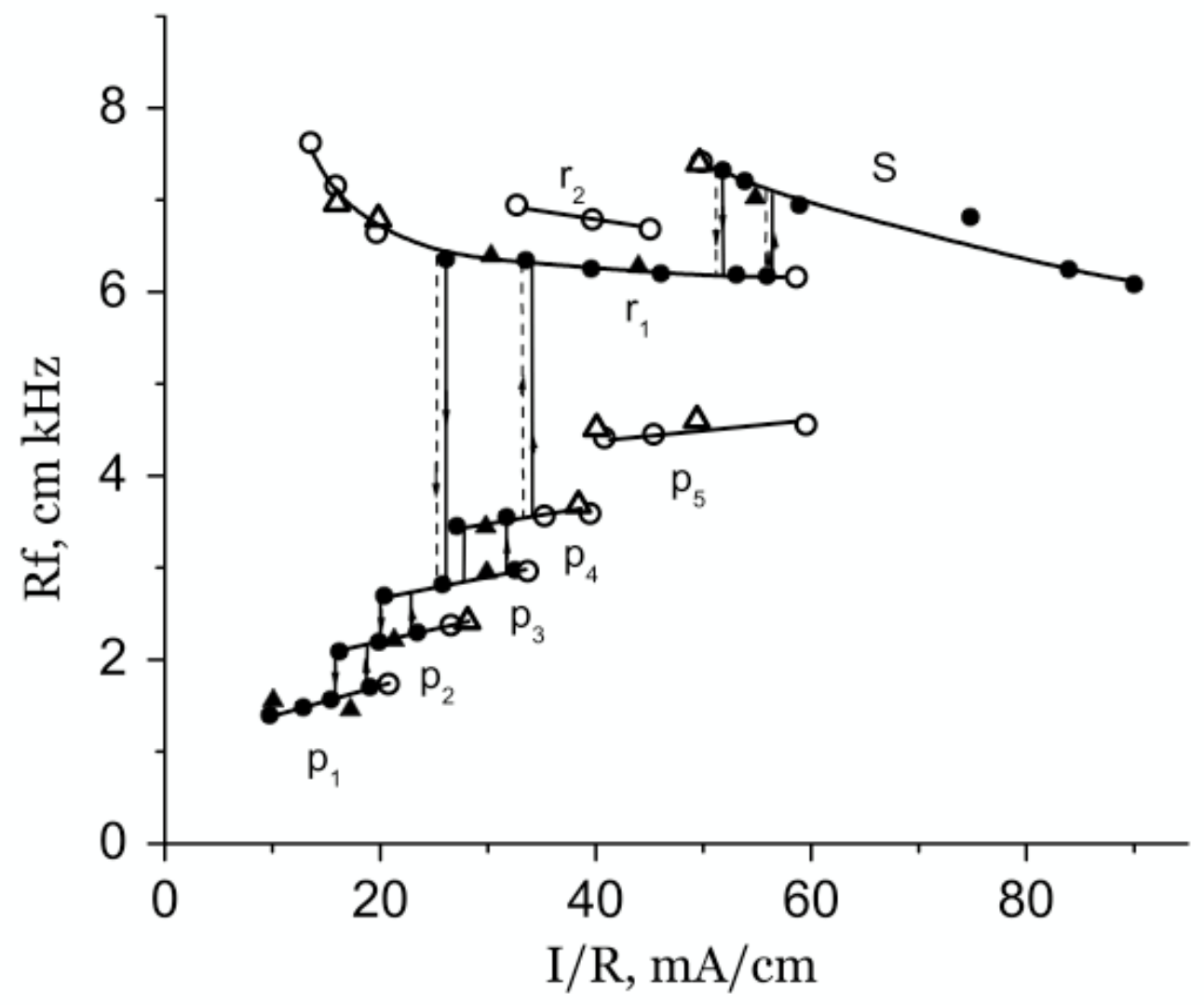

Figure 1.5: Striation frequency versus current in Ne at $p R=1$ Torr $\mathrm{cm}$. The work in this thesis was done at $p R=1.5$ Torr $\mathrm{cm}$, so this figure is representative of ionization wave behavior in the experiment. The different shapes represent different discharge tube radii, with $\bullet(\mathbf{\Lambda})$ representing $R=1.5(2.9) \mathrm{cm}$. The hollow shapes represent artificially driven waves. [From Kolobov (2006).] 


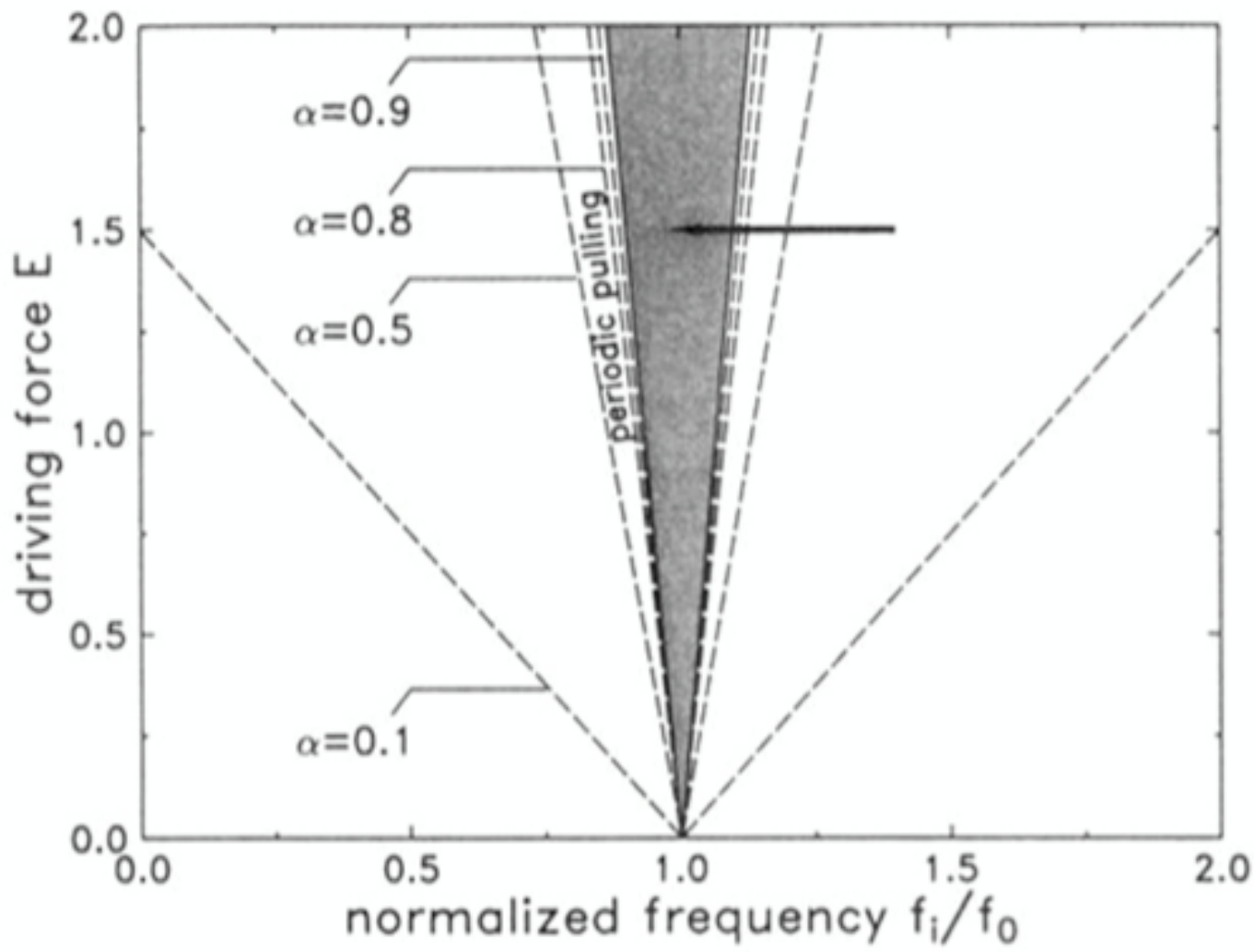

Figure 2.1: Schematic diagram of an Arnol'd tongue. This figure can be reproduced by calculating $\alpha$ using Eq. 2.6 and setting $2 a=15$. The dark inverted triangle (arrow) is the region where $\alpha>1$, the Arnol'd tongue. Periodic pulling is strongest very near the Arnol'd tongue, as indicated. [From Klinger et al. (1995).] 

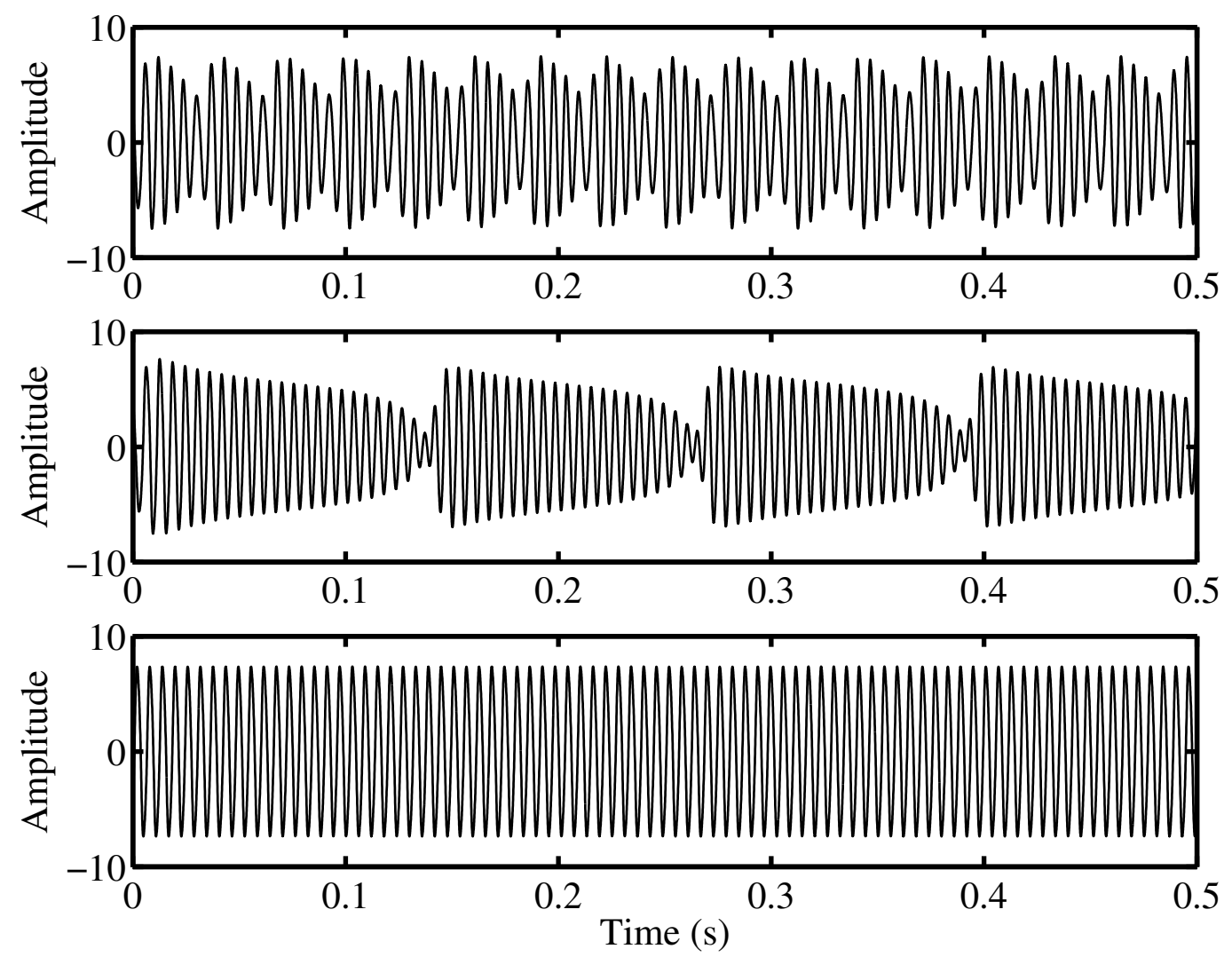

Figure 2.2: The time series behavior for three cases of driving frequency. Using the van der Pol equation (2.1) with $\epsilon=0.4, \beta=0.1, \omega_{0} / 2 \pi=160 \mathrm{~Hz}$ and $E=1.5, \omega_{i} / \omega_{0}$ was selected to demonstrate weak periodic pulling (top, $\omega_{i} / \omega_{0}=1.2157$ ), strong periodic pulling (center, $\omega_{i} / \omega_{0}=$ 1.1195), and entrainment (bottom, $\omega_{i} / \omega_{0}=1.05$ ). Parameters were chosen to approximately reproduce Figures 3-5 in Klinger et al. (1995). 

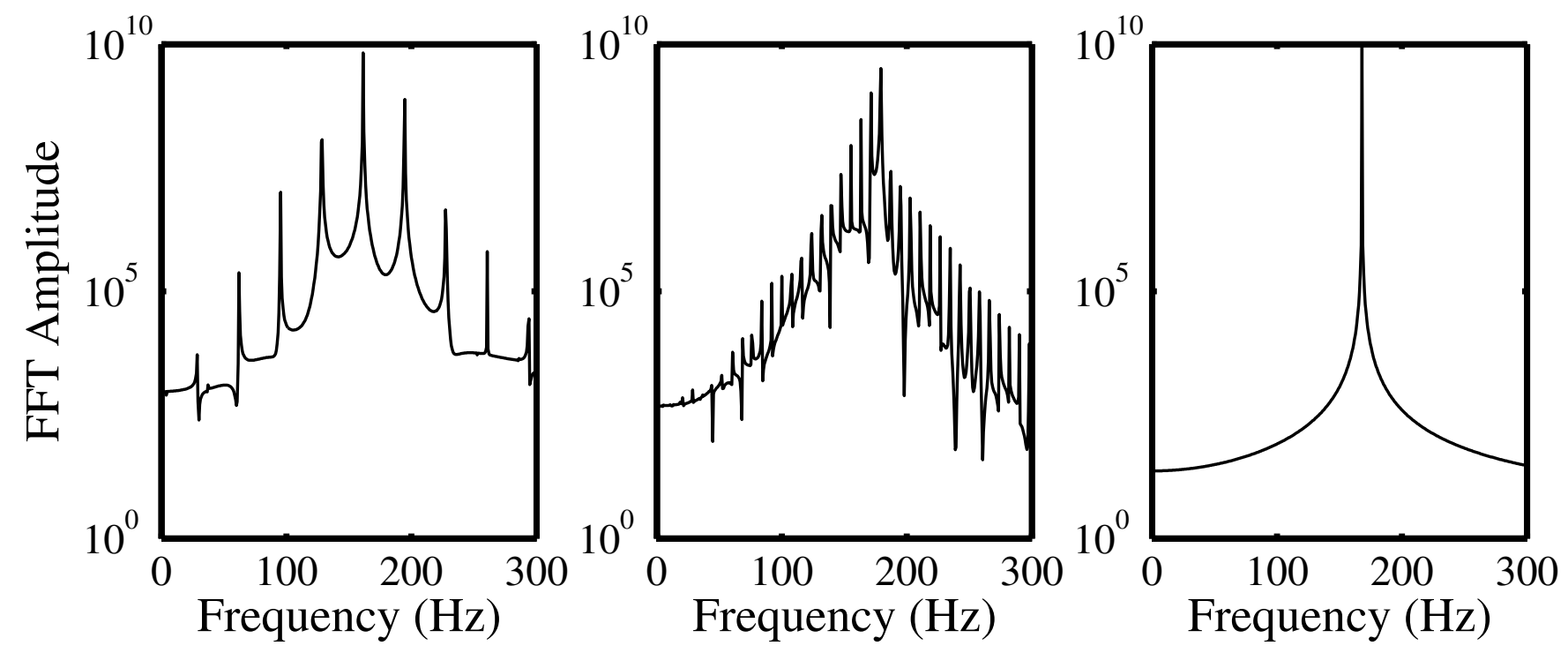

Figure 2.3: FFT amplitude of the time series shown in Figure 2.2. Using the van der Pol equation (2.1) with $\epsilon=0.4, \beta=0.1, \omega_{0} / 2 \pi=160 \mathrm{~Hz}$ and $E=1.5, \omega_{i} / \omega_{0}$ was selected to demonstrate weak periodic pulling (left panel, $\omega_{i} / \omega_{0}=1.2157$ ), strong periodic pulling (center panel, $\omega_{i} / \omega_{0}=1.1195$ ), and entrainment (right panel, $\omega_{i} / \omega_{0}=1.05$ ). Parameters were chosen to approximately reproduce Figures 3-5 in Klinger et al. (1995). 
(a)

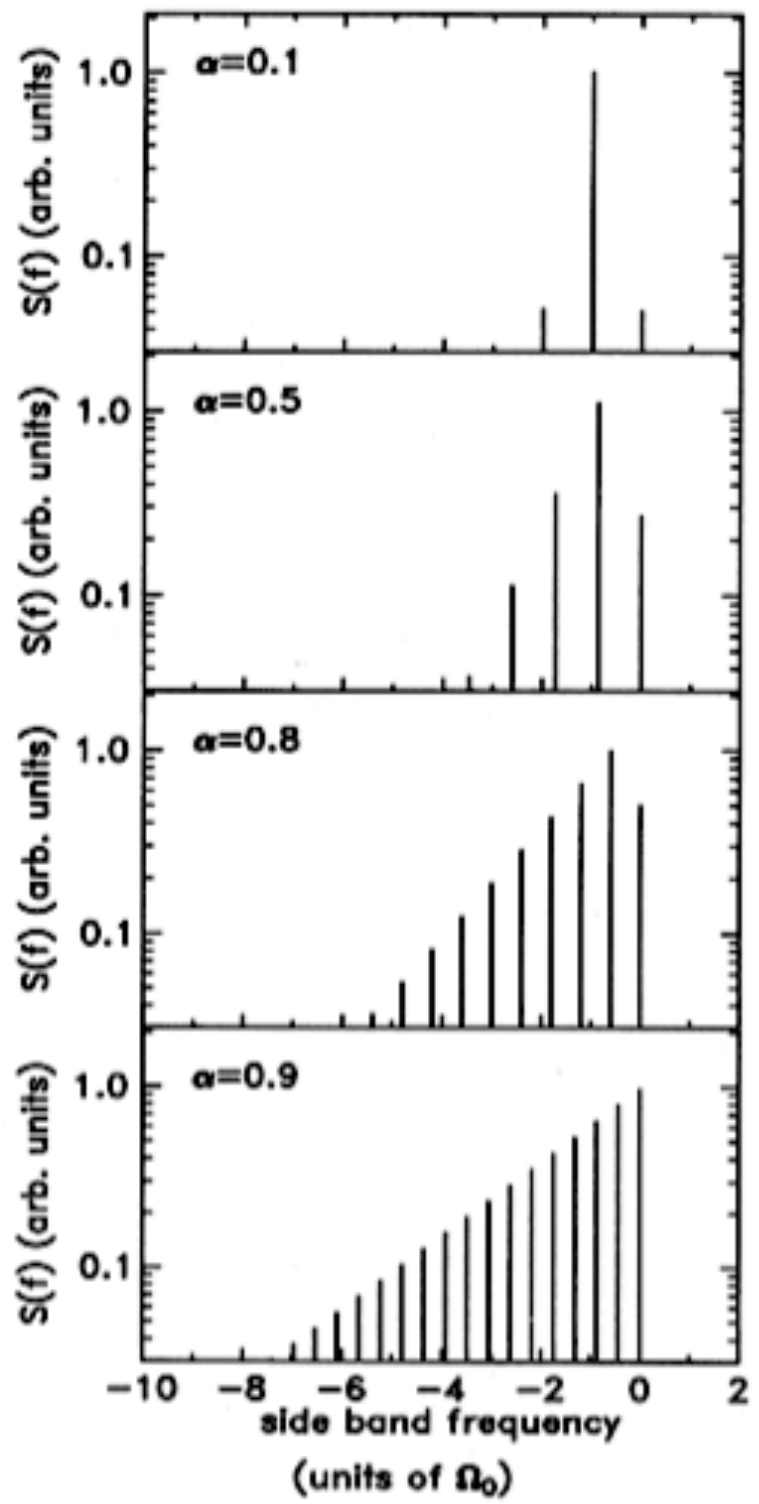

(b)

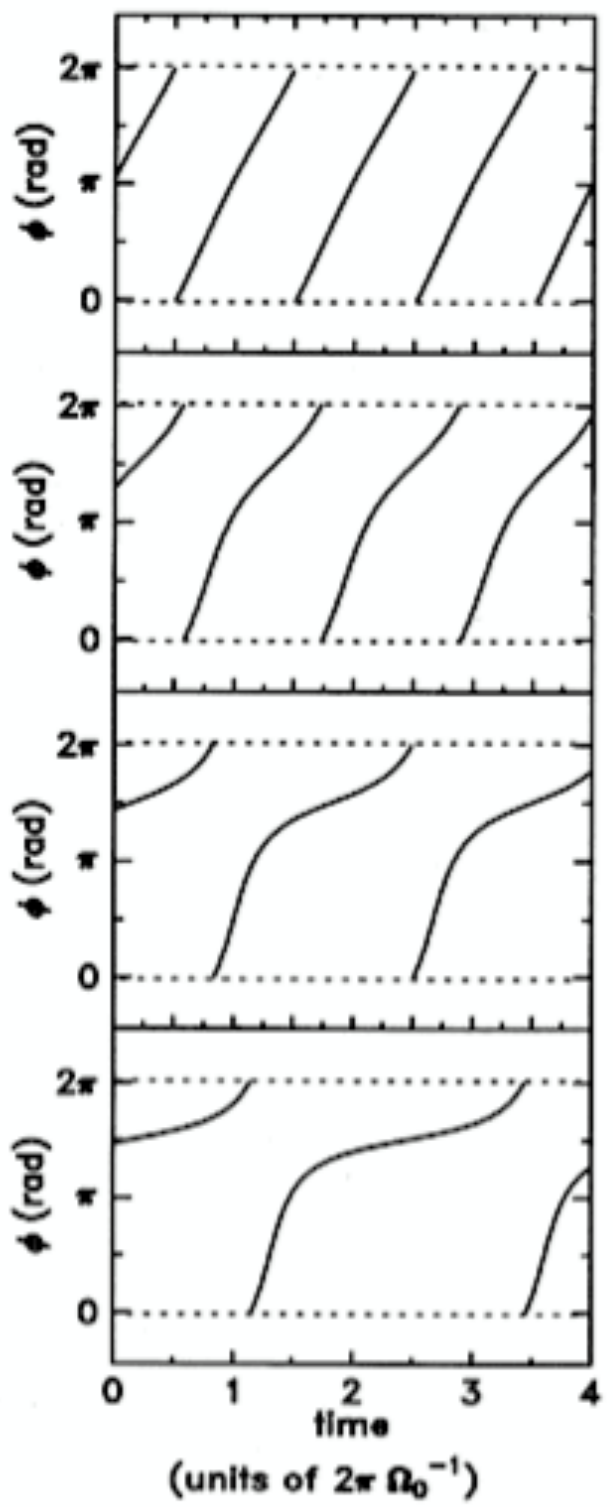

Figure 2.4: Four cases of $\alpha$, showing power spectra sideband structure (a) and phase development (b) as a function of time. [From Klinger et al. (1995).] 


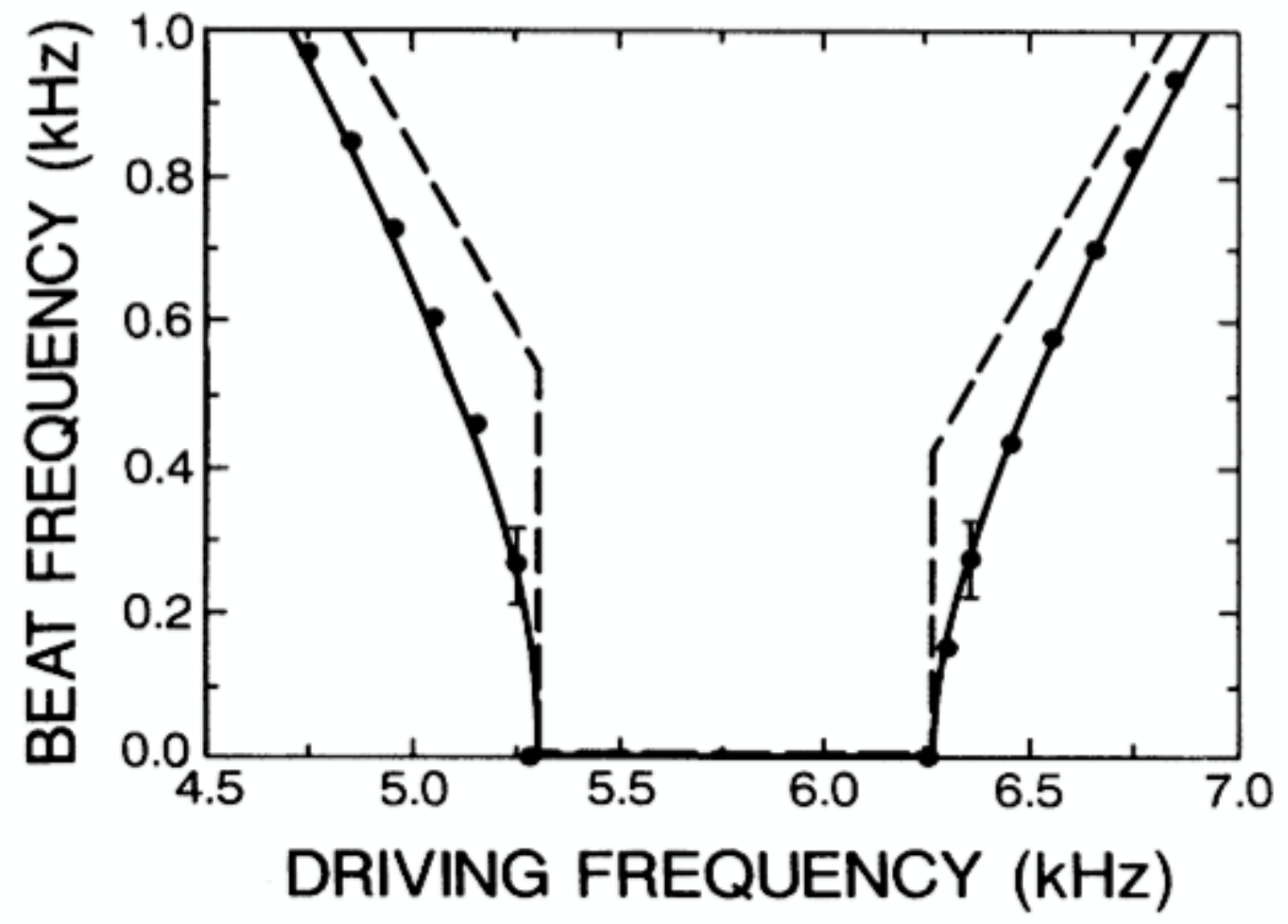

Figure 2.5: Arnol'd tongue cross section. Figure from Koepke and Hartley (1991), using data from a UJT circuit. 

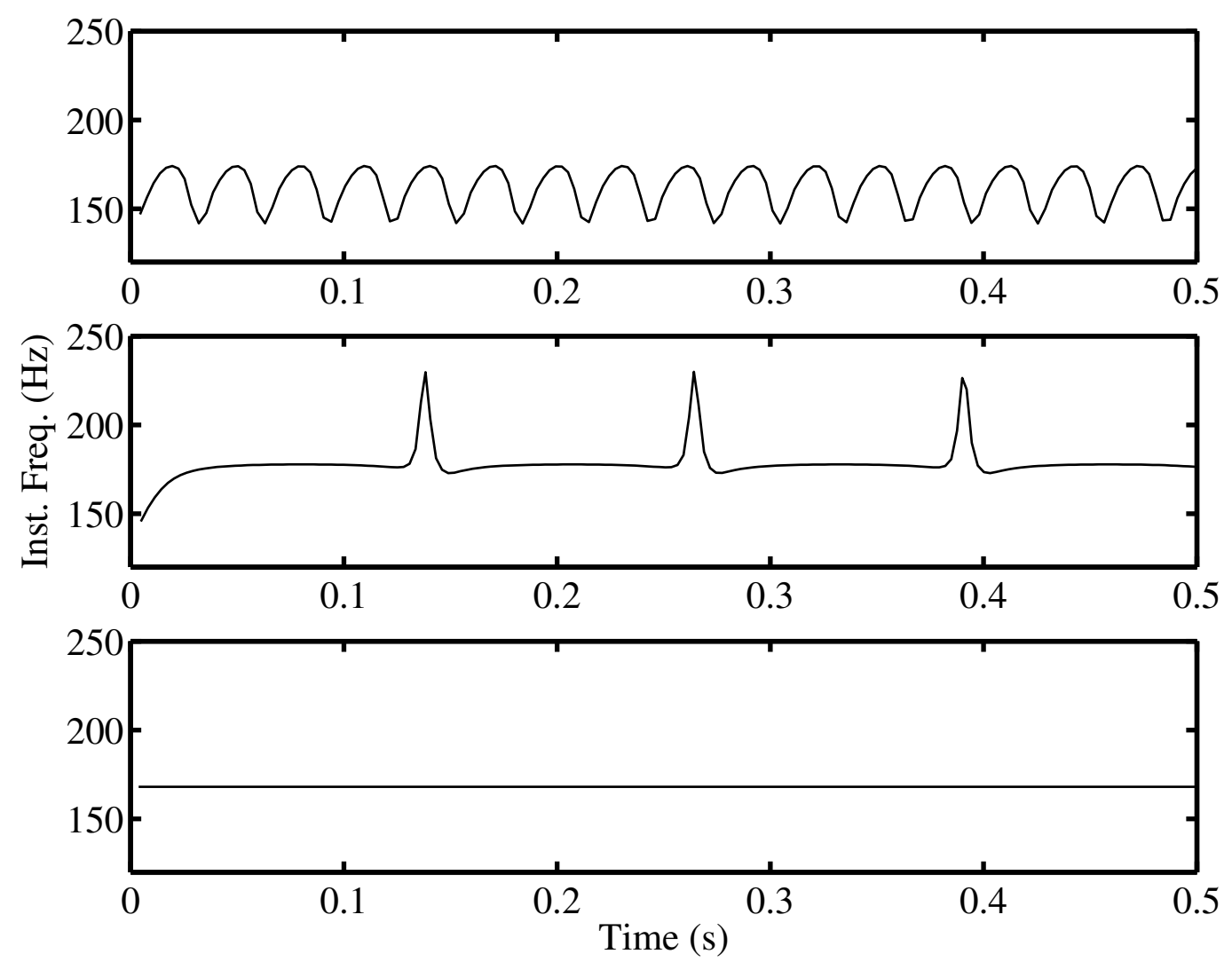

Figure 2.6: Instantaneous frequency of the time series shown in Figure 2.2. Using the van der Pol equation (2.1) with $\epsilon=0.4, \beta=0.1, \omega_{0} / 2 \pi=160 \mathrm{~Hz}$ and $E=1.5, \omega_{i} / \omega_{0}$ was selected to demonstrate weak periodic pulling (top, $\omega_{i} / \omega_{0}=1.2157$ ), strong periodic pulling (center, $\omega_{i} / \omega_{0}=$ 1.1195), and entrainment (bottom, $\omega_{i} / \omega_{0}=1.05$ ). 

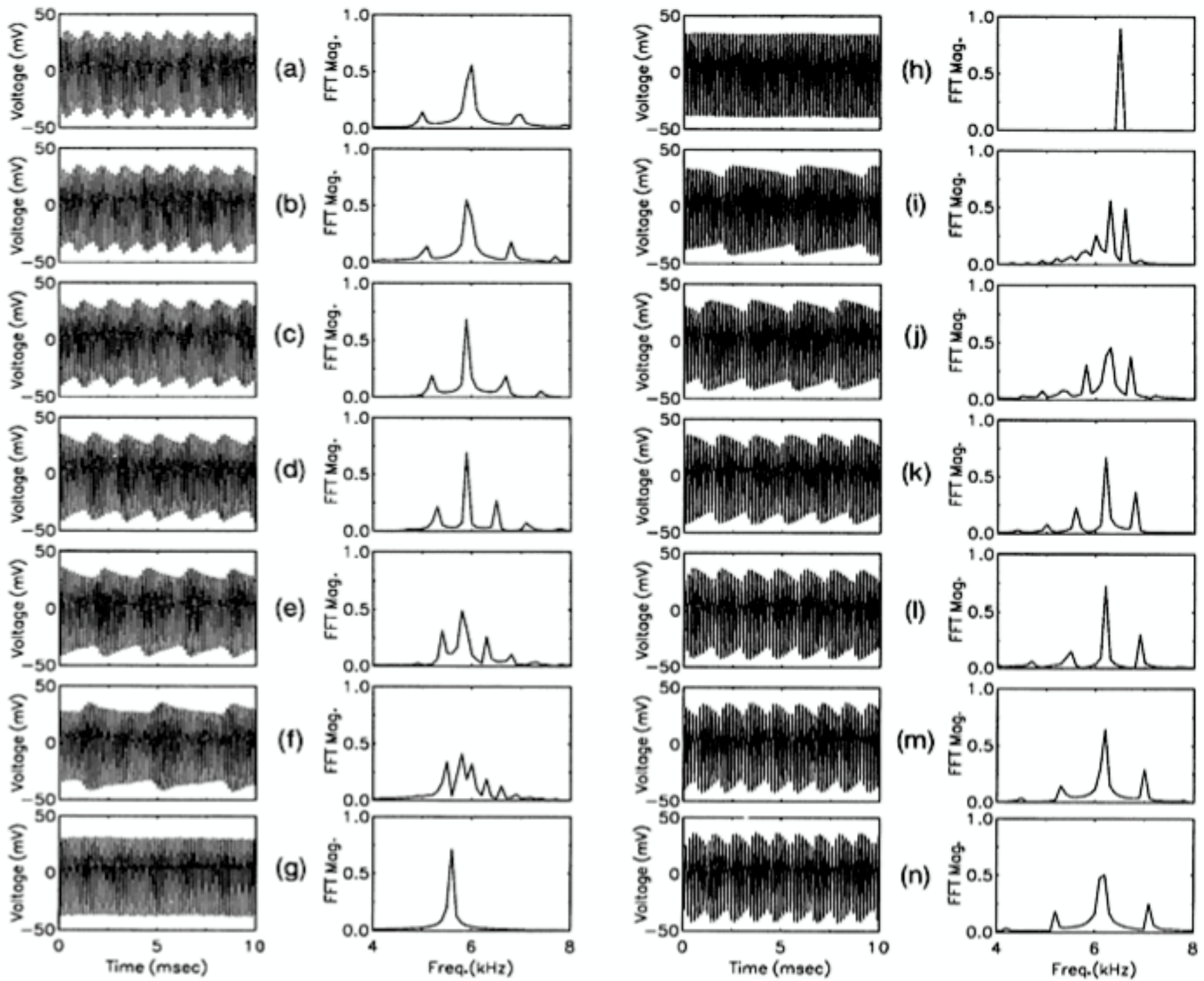

Figure 2.7: Time series and FFT as driving frequency is adjusted from below the natural frequency (a through $\mathrm{f}$ ) to above the natural frequency (i through $\mathrm{n}$ ). Periodic pulling is evident on both sides of entrainment (g and h). Data from a UJT circuit. Figure from Koepke and Hartley (1991). 


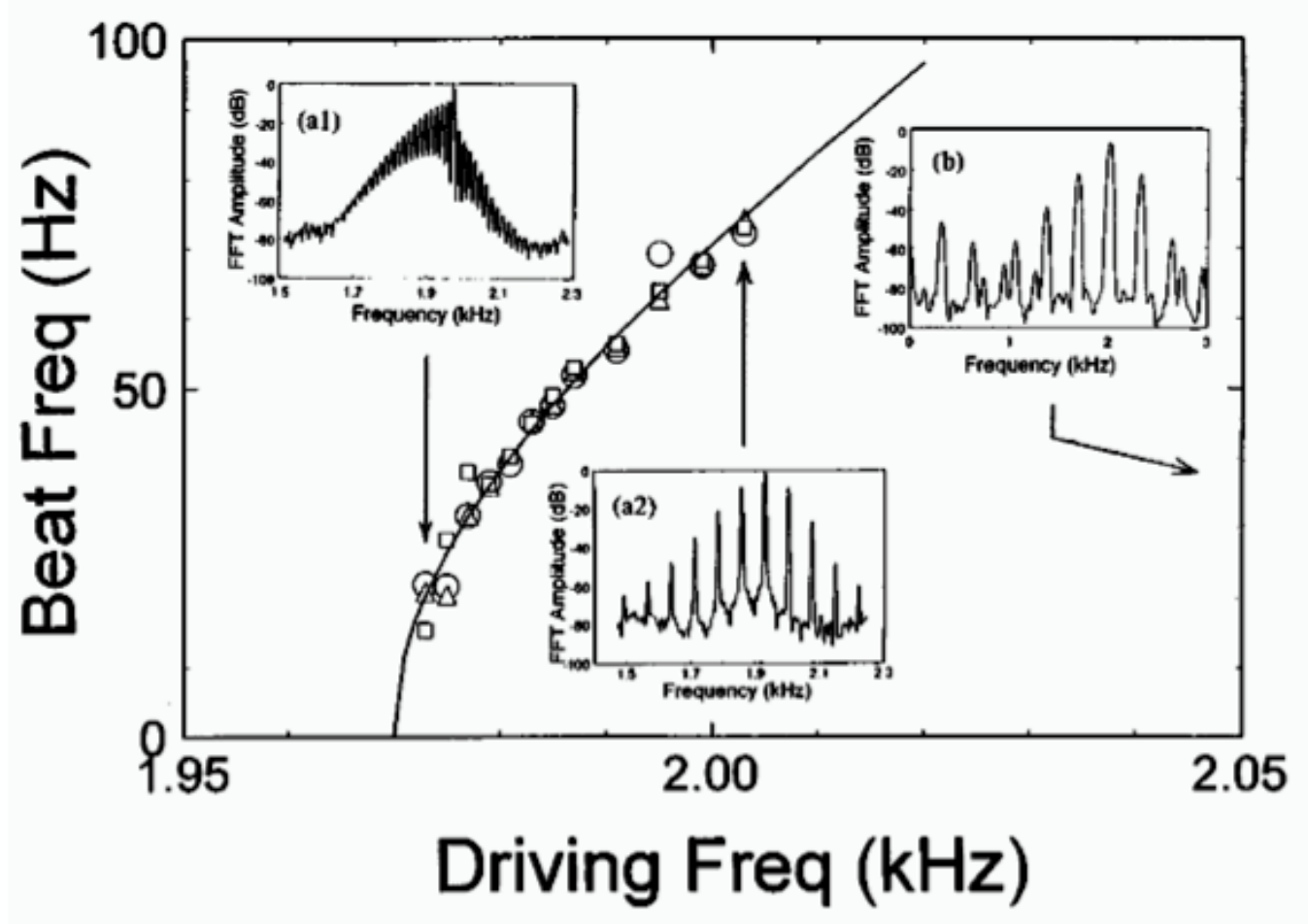

Figure 2.8: Temporal periodic pulling versus spatio-temporal periodic pulling. Many examples of temporal periodic pulling (a1 and a2, and points on the curve) fit nicely on a predicted curve of beat frequency versus driving frequency. Koepke et al. (1996) estimate that the spatio-temporal case (b) has a similar driving strength to the temporal cases, but does so from a greater frequency separation. Therefore, it has a much larger effective driving amplitude $M$ based on Eq. (2.8). Figure from Koepke et al. (1996), using data from a neon glow discharge. 

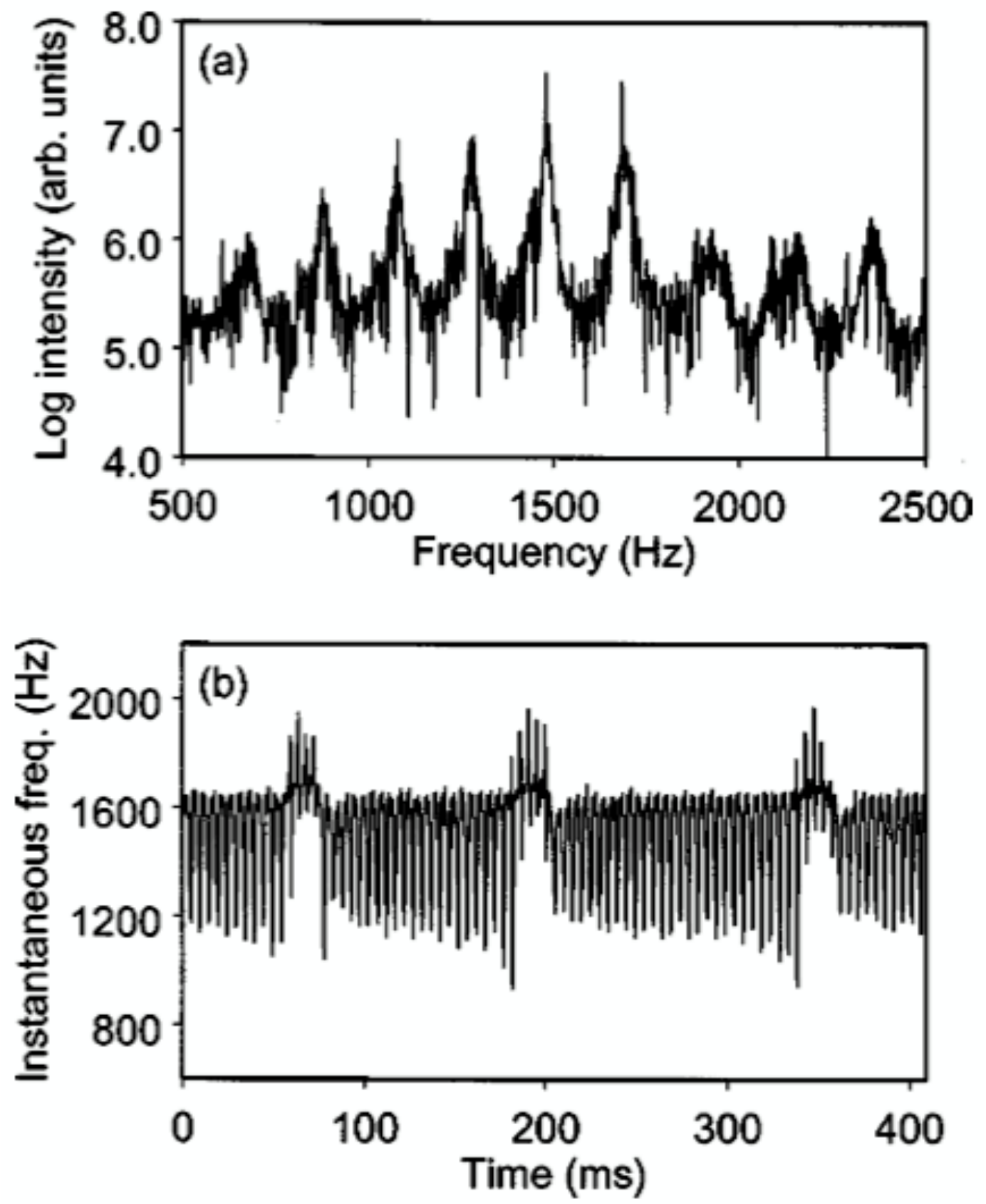

Figure 2.9: Dynamics modulation. The instantaneous frequency clearly and periodically hops up to the $1.75 \mathrm{kHz}$ mode and back down to the $1.5 \mathrm{kHz}$ mode. Figure from Weltmann et al. (2000), using data from a neon glow discharge. 


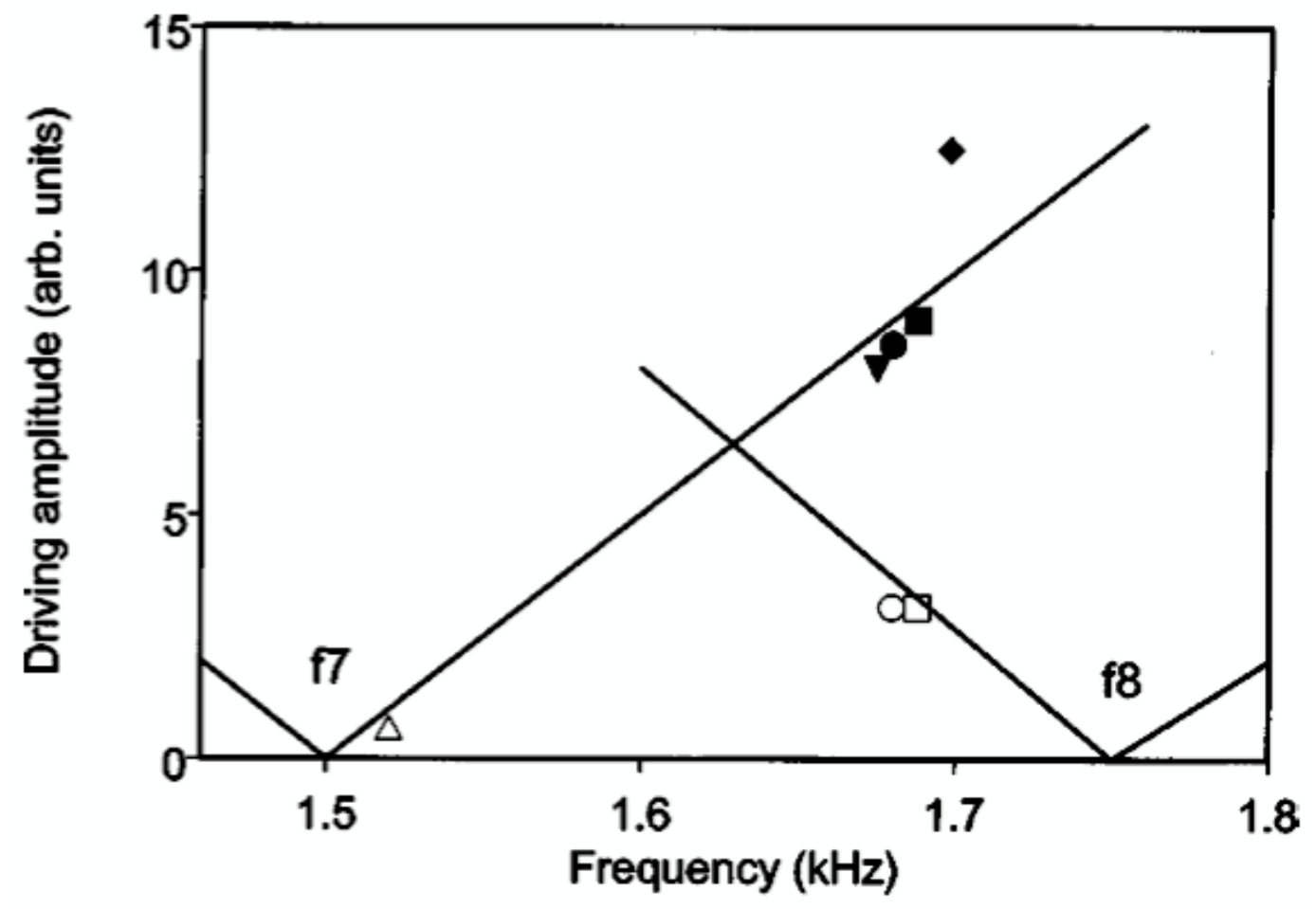

Figure 2.10: Dynamics phase diagram for a multimode oscillator. The $\triangle$ represents a case of temporal periodic pulling. The $\boldsymbol{\nabla}$ represents spatiotemporal periodic pulling with no dynamics modulation. The represents entrainment of the higher mode, with no dynamics modulation. The - and cases represent two cases of dynamics modulation when combined with their counterparts, $\circ$ and the $\square$. See text for a complete explanation. From Weltmann et al. (2000). 


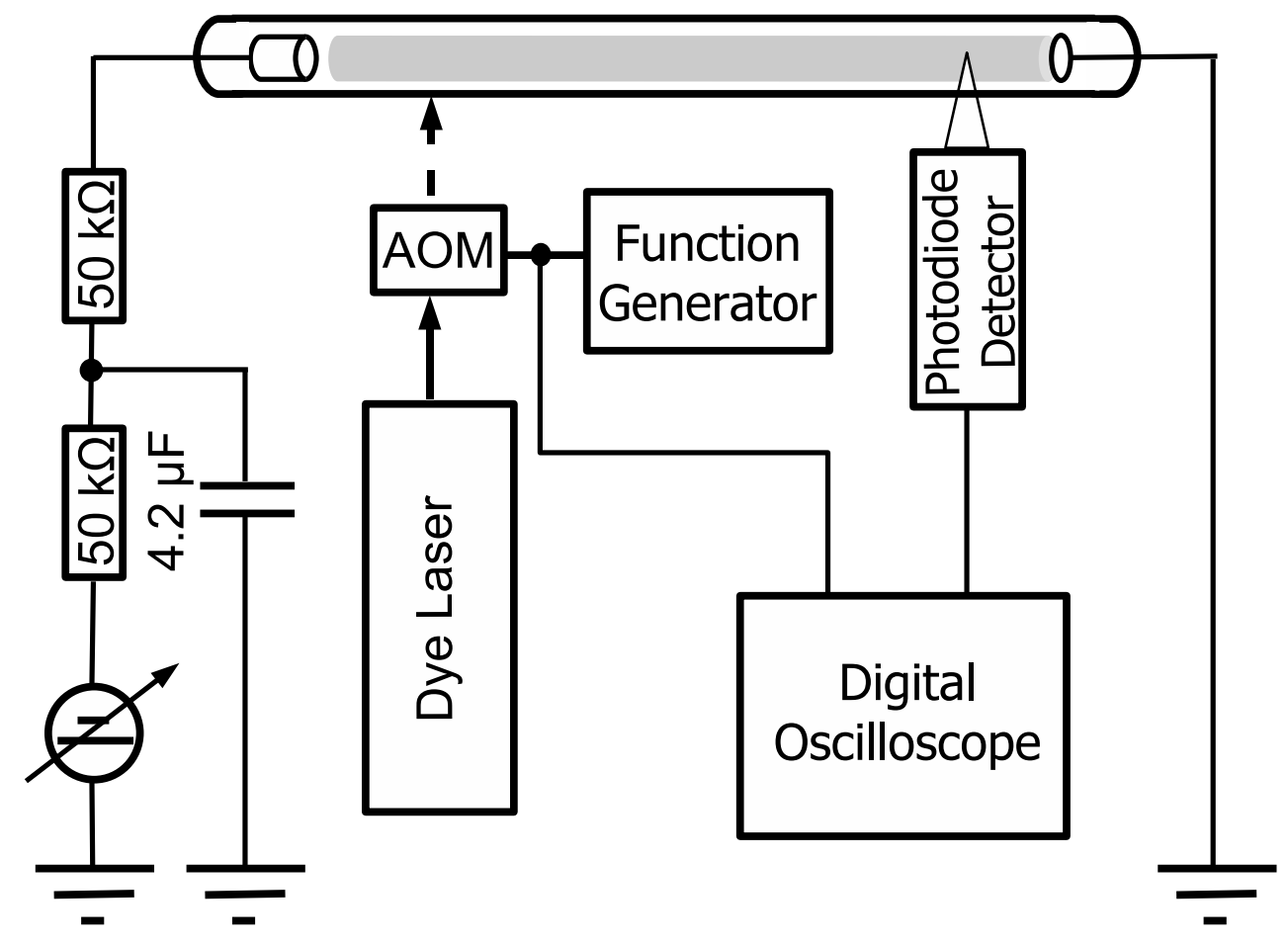

Figure 3.1: The experimental arrangement. The cathode of the discharge tube is on the left, and the anode is on the right. A $10 \Omega$ resistor (not pictured) was placed between the anode and ground, and the voltage across this resistor was used to measure the undriven modes for Figures 4.1 and 4.3. More description of the experimental arrangement can be found in the text. 


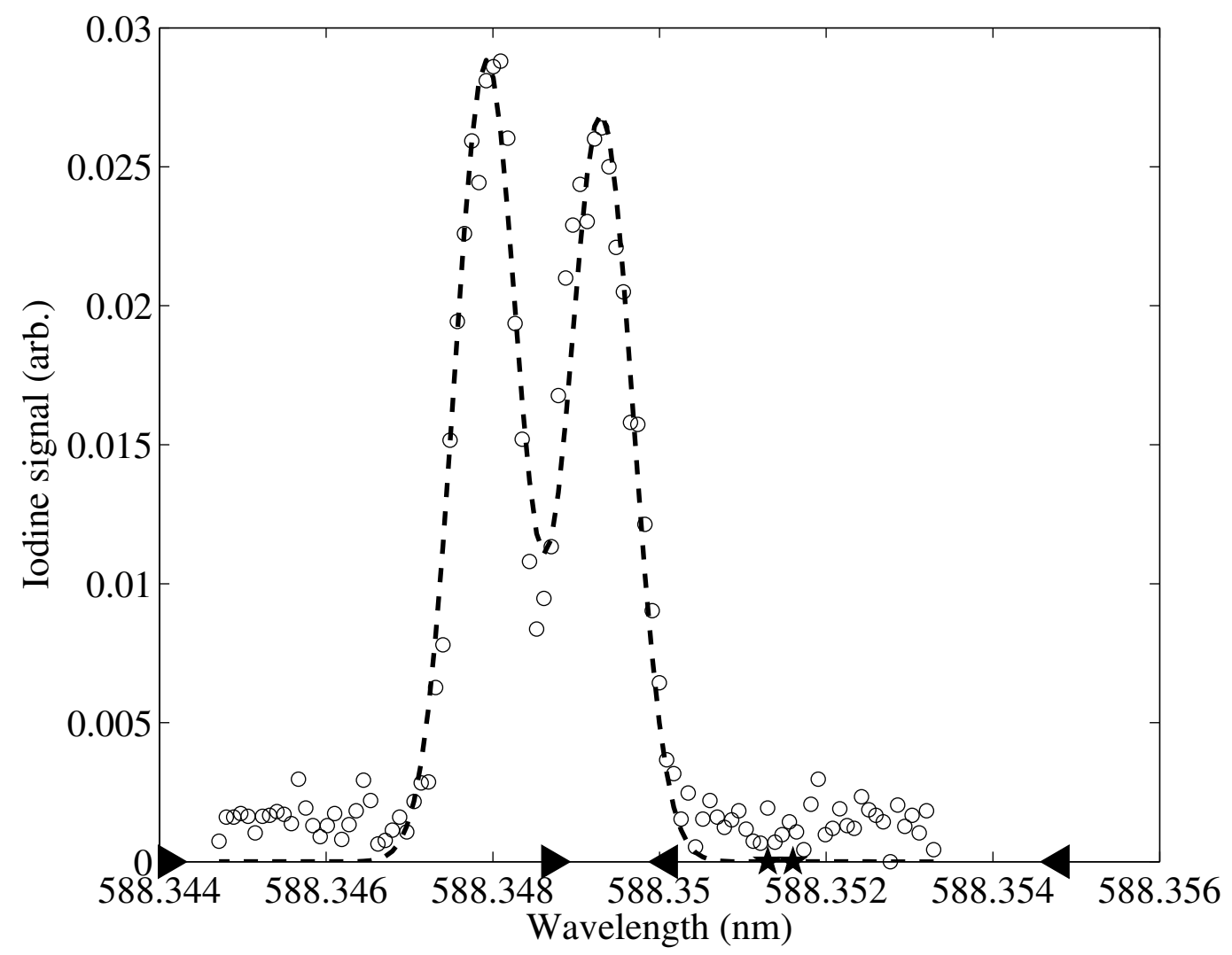

Figure 3.2: Iodine cell calibration. Output of iodine cell (o) with fit (dashed line) to nearby lines from iodine atlas, which establishes the wavelength range. The outer triangles on the axis represent the approximate wavelength extremes at which the laser just begins to affect the discharge. Between the inner pair of triangles, the laser can entrain any driving frequency in the discharge. The two five-pointed stars have been placed at representative wavelengths where dynamics modulation has been observed. 

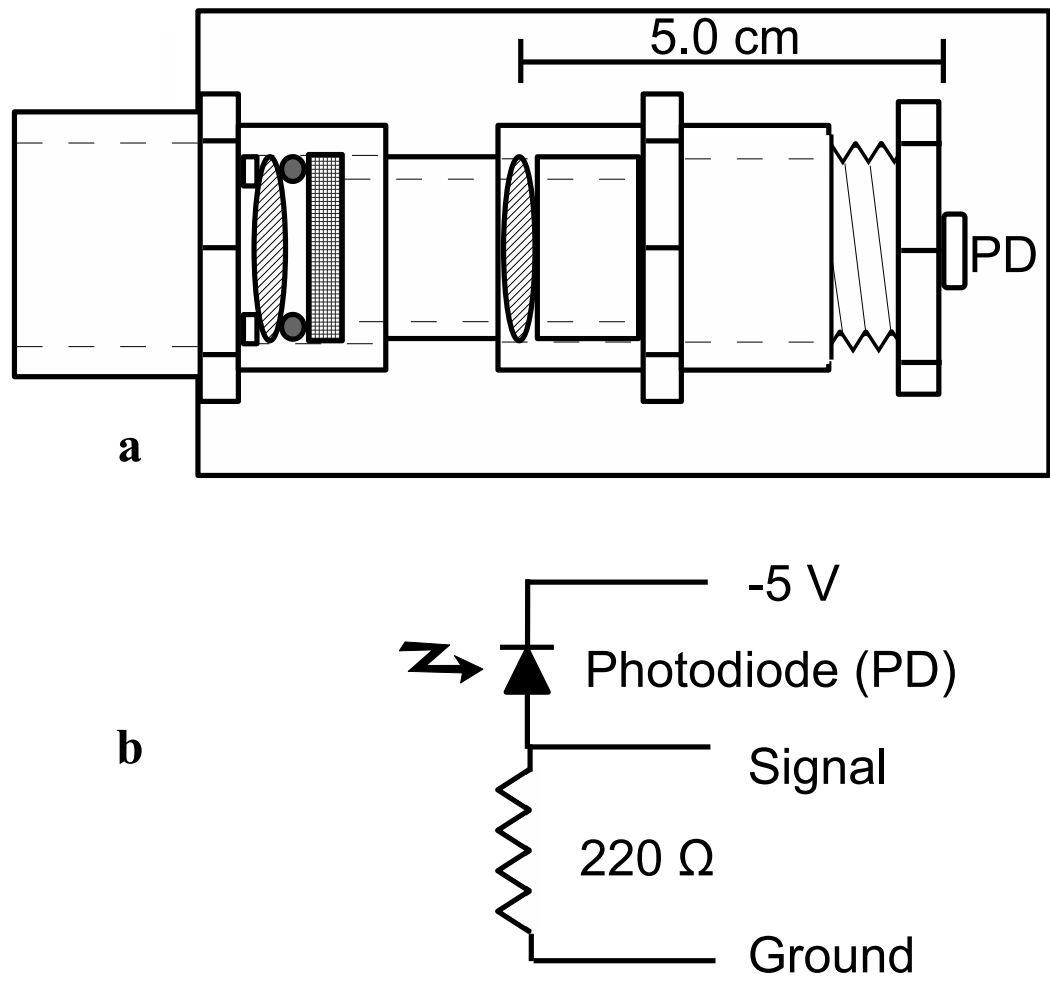

Figure 3.3: Optics (a) and electronics (b) of the photodiode circuit. Light would enter from left to right, and pass through two $50 \mathrm{~mm}$ focal length lenses. Between the lenses, there is a $640.1 \mathrm{~nm}$ (4 $\mathrm{nm}$ bandwidth) filter. More details about the design appear in the text. 


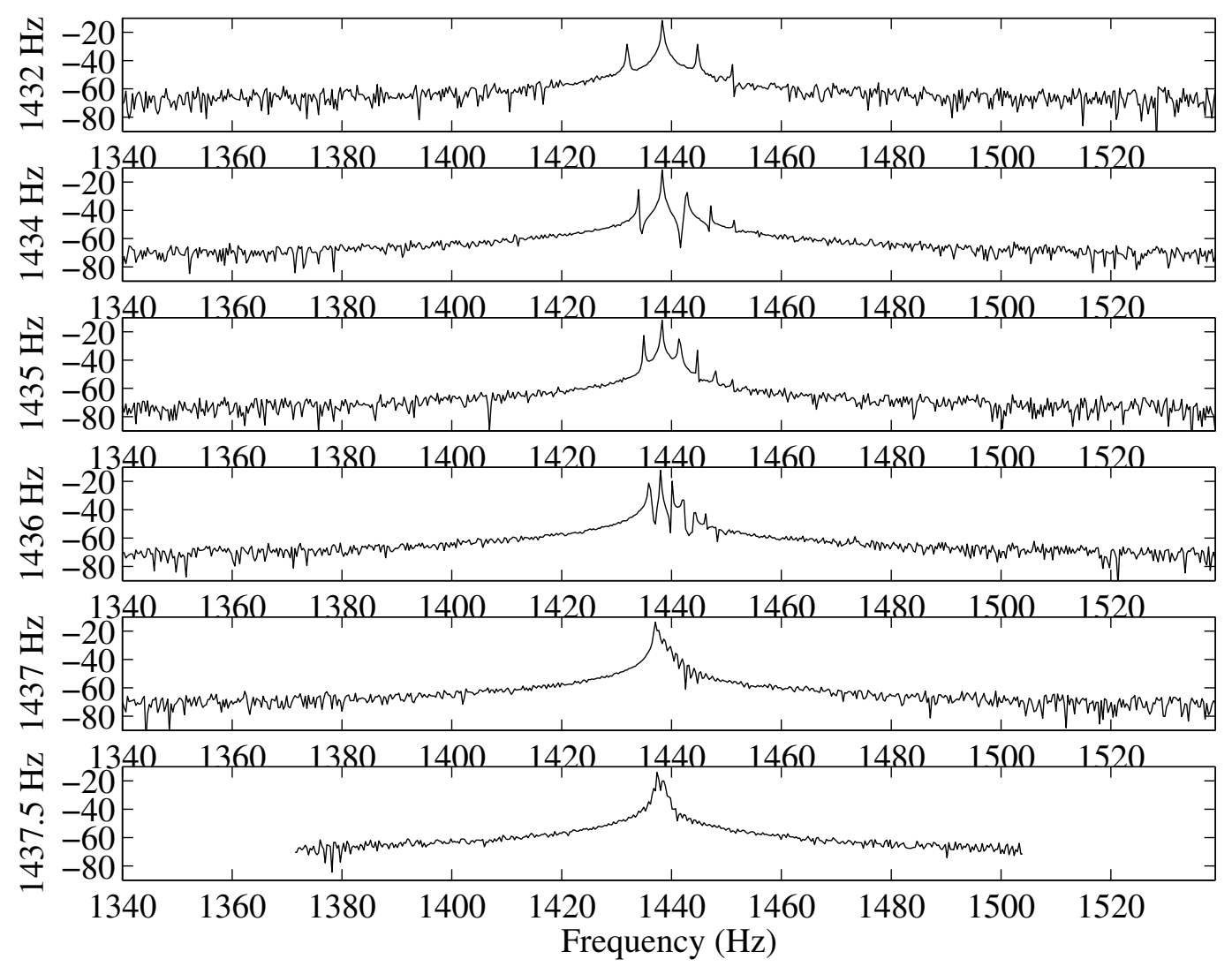

Figure 3.4: Identifying the edge of an Arnol'd tongue from the power spectra. The $y$-axis shows the logarithm of the magnitude of the FFT. Mode frequency is around $1440 \mathrm{~Hz}$. The driving frequency is shown on the left side of each of the power spectra, and it is driving the mode from below. Peaks are visible at the driving frequency in each panel. Periodic pulling increases going down the figure until, finally, in the bottom panel, the oscillation is entrained to the driving frequency. 

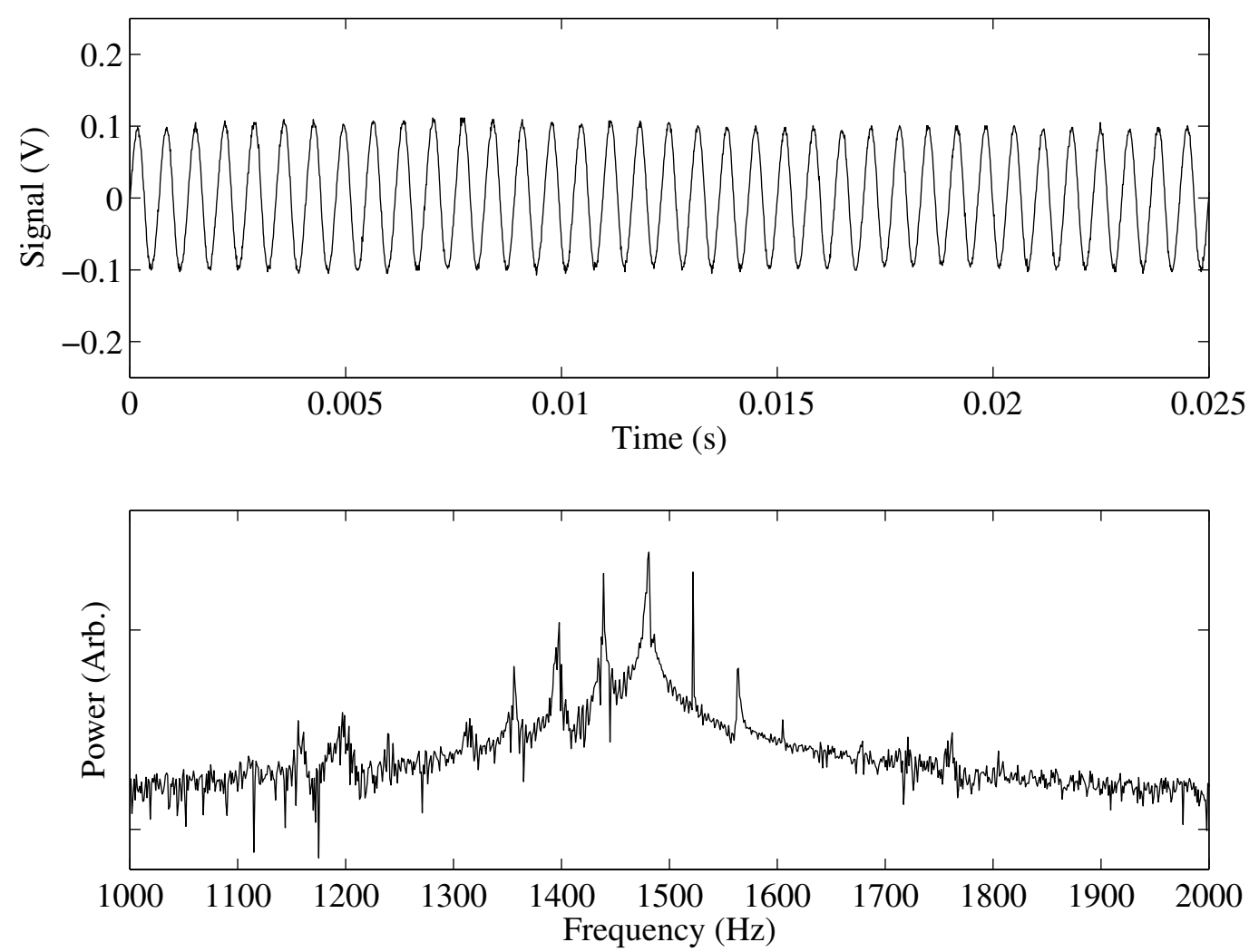

Figure 3.5: Spectrum of temporal periodic pulling. The discharge current was set to $7.87 \mathrm{~mA}$. The driving frequency (here at $1522 \mathrm{~Hz}$ ) is above the undriven oscillation frequency $(1466 \mathrm{~Hz})$. 

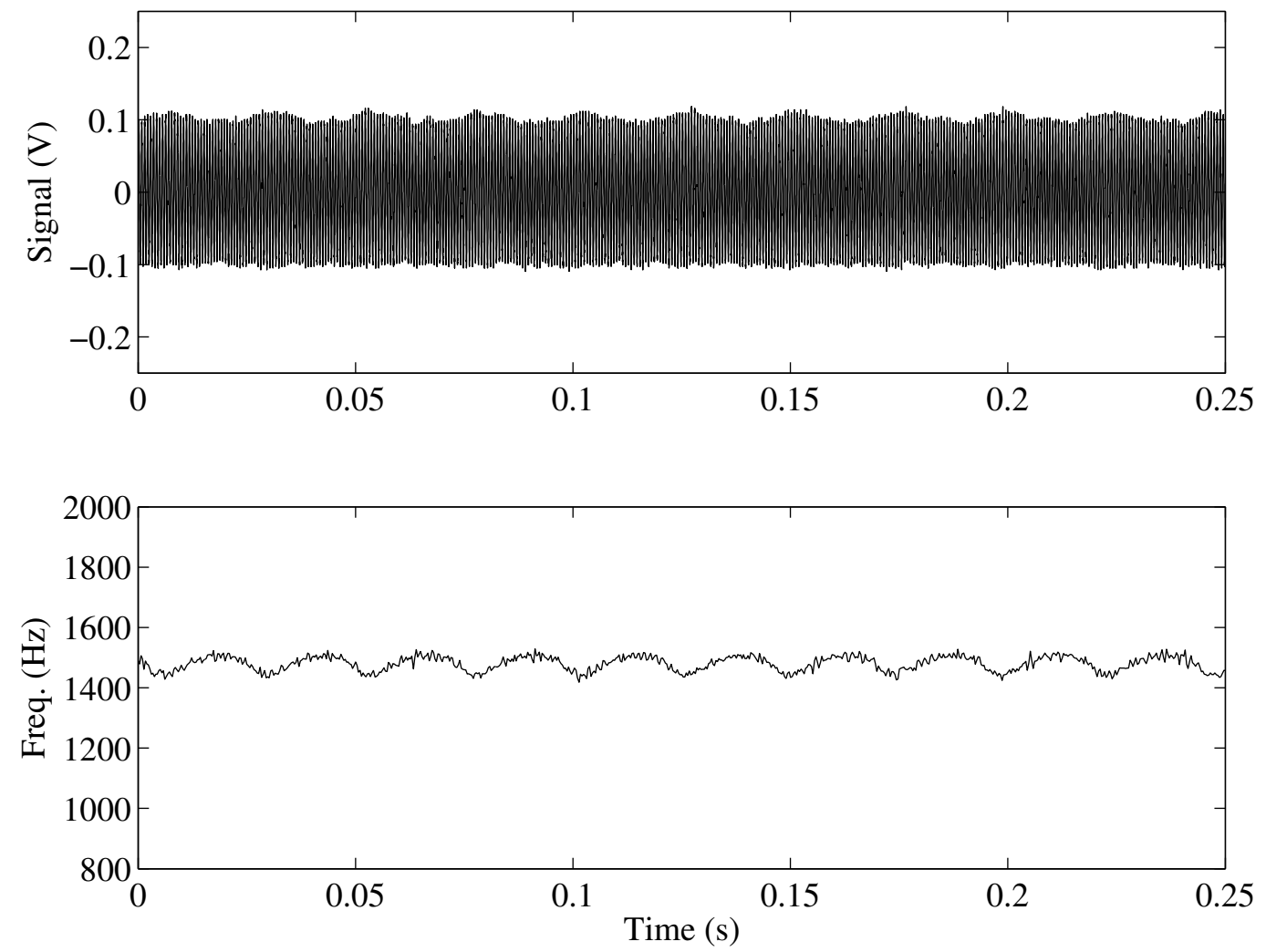

Figure 3.6: Instantaneous frequency series for temporal periodic pulling. The instantaneous frequency (bottom) and the corresponding time series is shown (top) is shown. The instantaneous frequency has a clear beat shown by arcs in a concave-downward orientation. While the oscillations occur so fast that they darken the entire region, it is clear that their overall amplitude is modulated along with the frequency. This figure displays the same data as Figure 3.5, so the driving frequency is aobve the dominant mode frequency. 

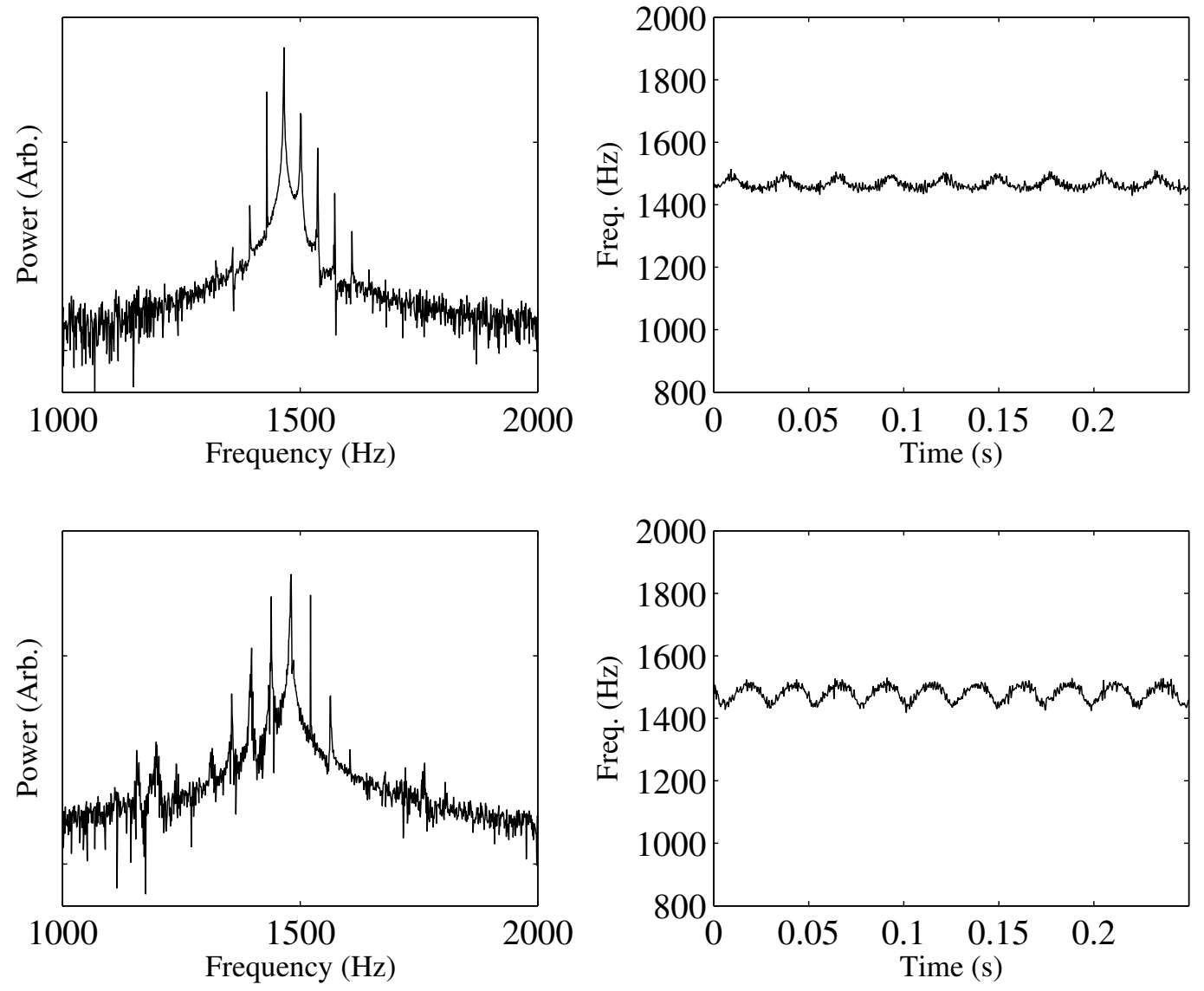

Figure 3.7: Temporal periodic pulling from each side. The case of Figures 3.5 and 3.6 (bottom), which had driving frequency $(1522 \mathrm{~Hz})$ above the mode frequency $(1466 \mathrm{~Hz})$, is shown alongside another case (top), with the driving frequency $(1430 \mathrm{~Hz})$ below the mode frequency $(1470 \mathrm{~Hz})$. Here the instantaneous frequency beat period arc appears concave up. Also clear from this figure is that the sidebands appear on the opposite side of the oscillation from the driving frequency. $\Omega$, determined from sideband separation, is $35.8 \mathrm{~Hz}$ (top) and $41.6 \mathrm{~Hz}$ (bottom). 

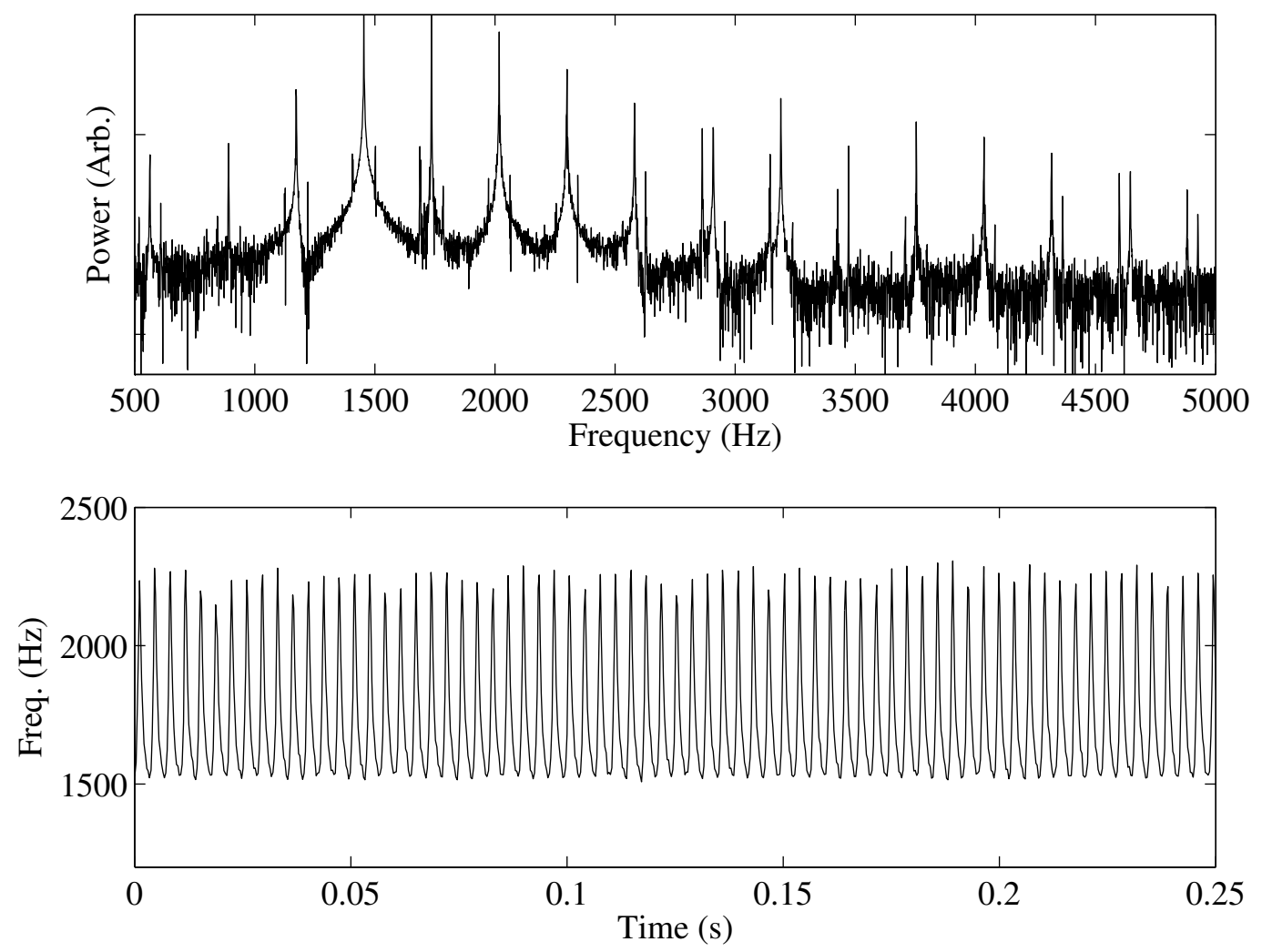

Figure 3.8: Signature of spatiotemporal periodic pulling. In the top half of the figure, the power spectrum is shown. The instantaneous frequency is shown on the bottom. The periodic pulling, evident by the range of the frequency oscillation, is much stronger than it was in the case of Figures 3.5 to 3.7 . 

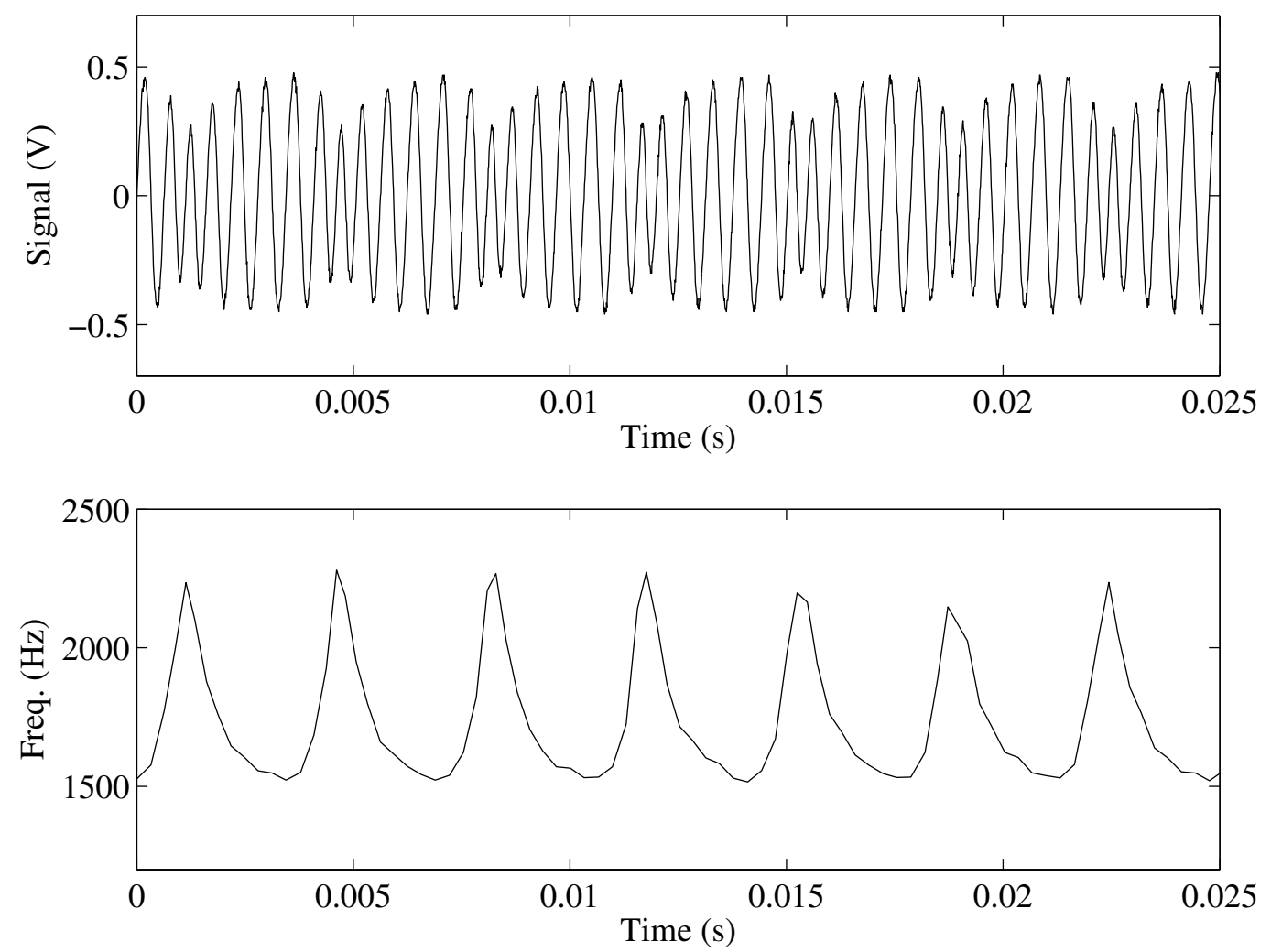

Figure 3.9: Close up of spatiotemporal time series and instantaneous frequency series. 

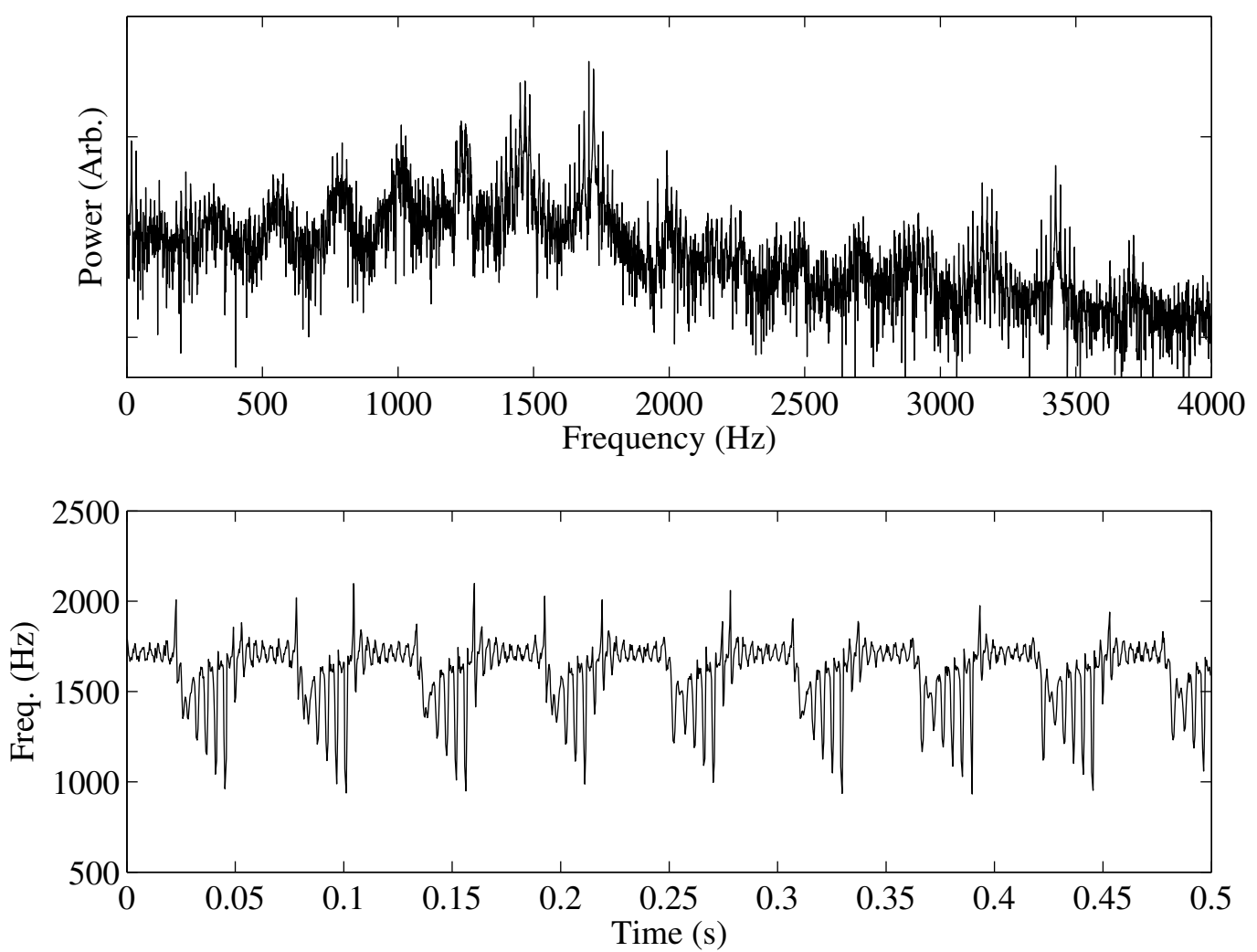

Figure 3.10: Signature of dynamics modulation. The distinct "hairy" spectrum (top) and the visible mode changes in the instantaneous frequency series (bottom). The undriven frequency is $1450 \mathrm{~Hz}$, and the driving frequency was set to $1704 \mathrm{~Hz}$. 

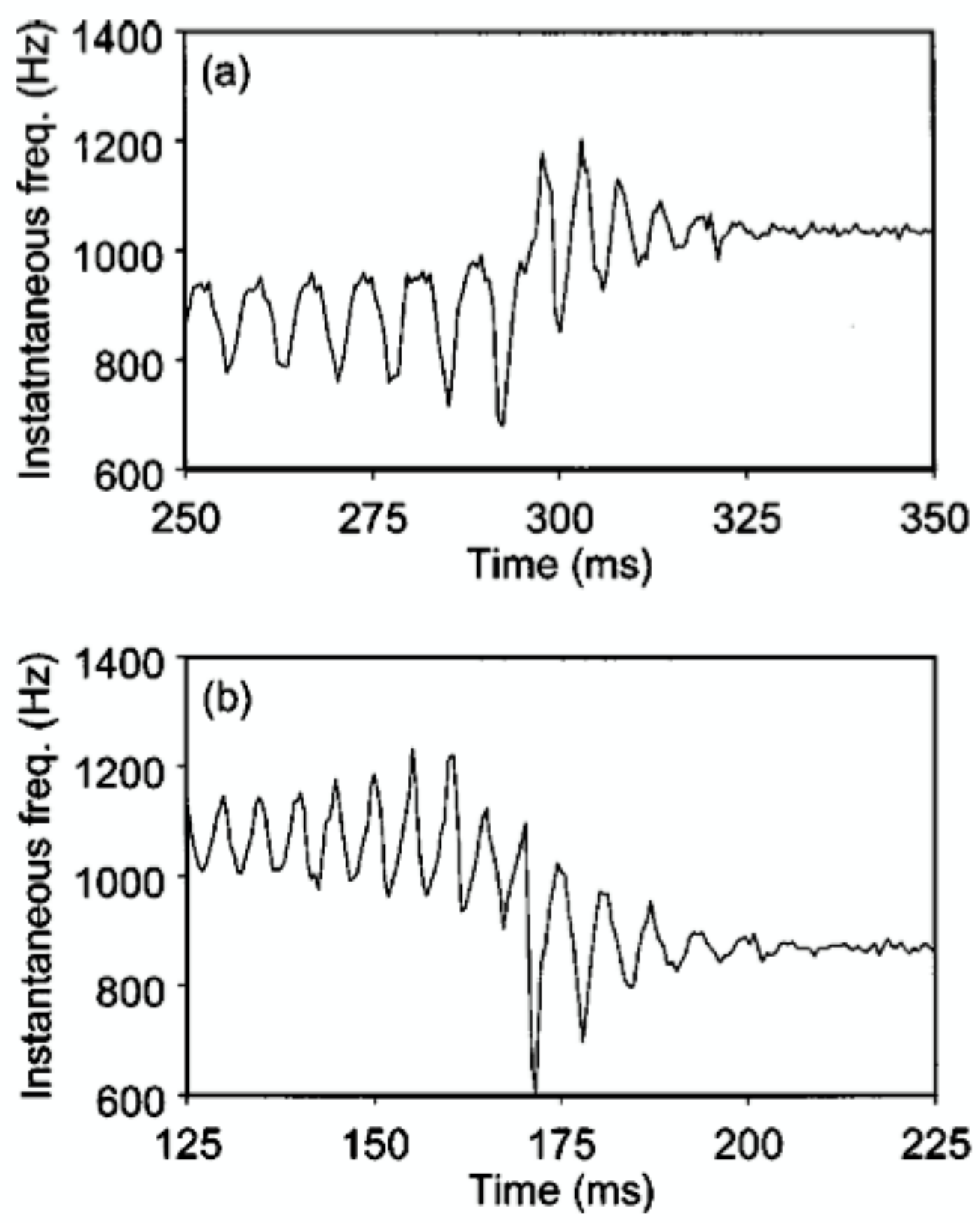

Figure 3.11: Instantaneous frequency of undriven mode transitions. [From Weltmann et al. (2000)]. 


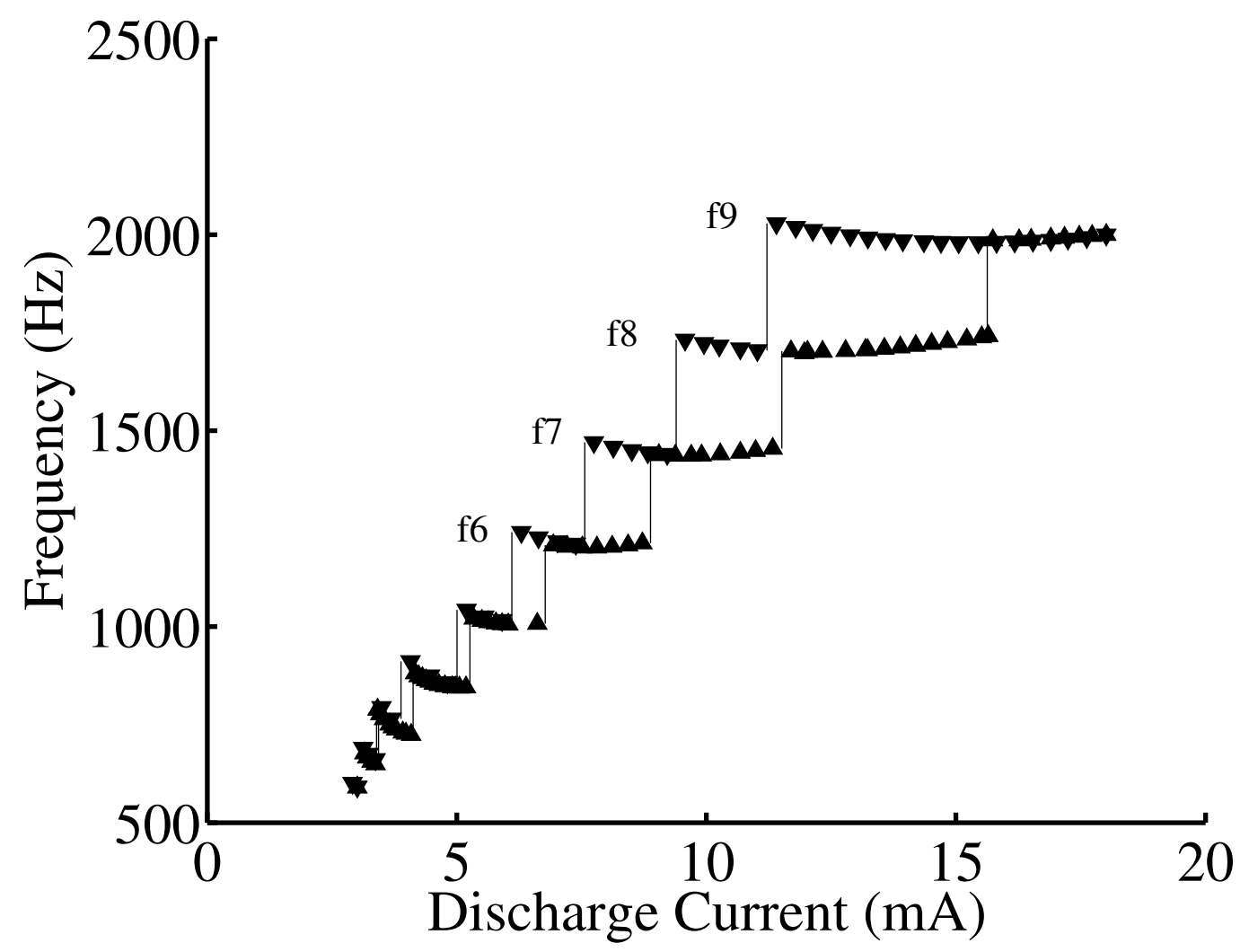

Figure 4.1: Ionization wave modes as a function of discharge current. Vertical lines indicate hysteresis. The right-hand (left-hand) line of each pair represents the discharge current for which the mode changes as the current is increasing (decreasing). The triangles indicate mode frequencies measured between increasing $(\mathbf{\Lambda})$ or decreasing $(\boldsymbol{\nabla})$ the current. 


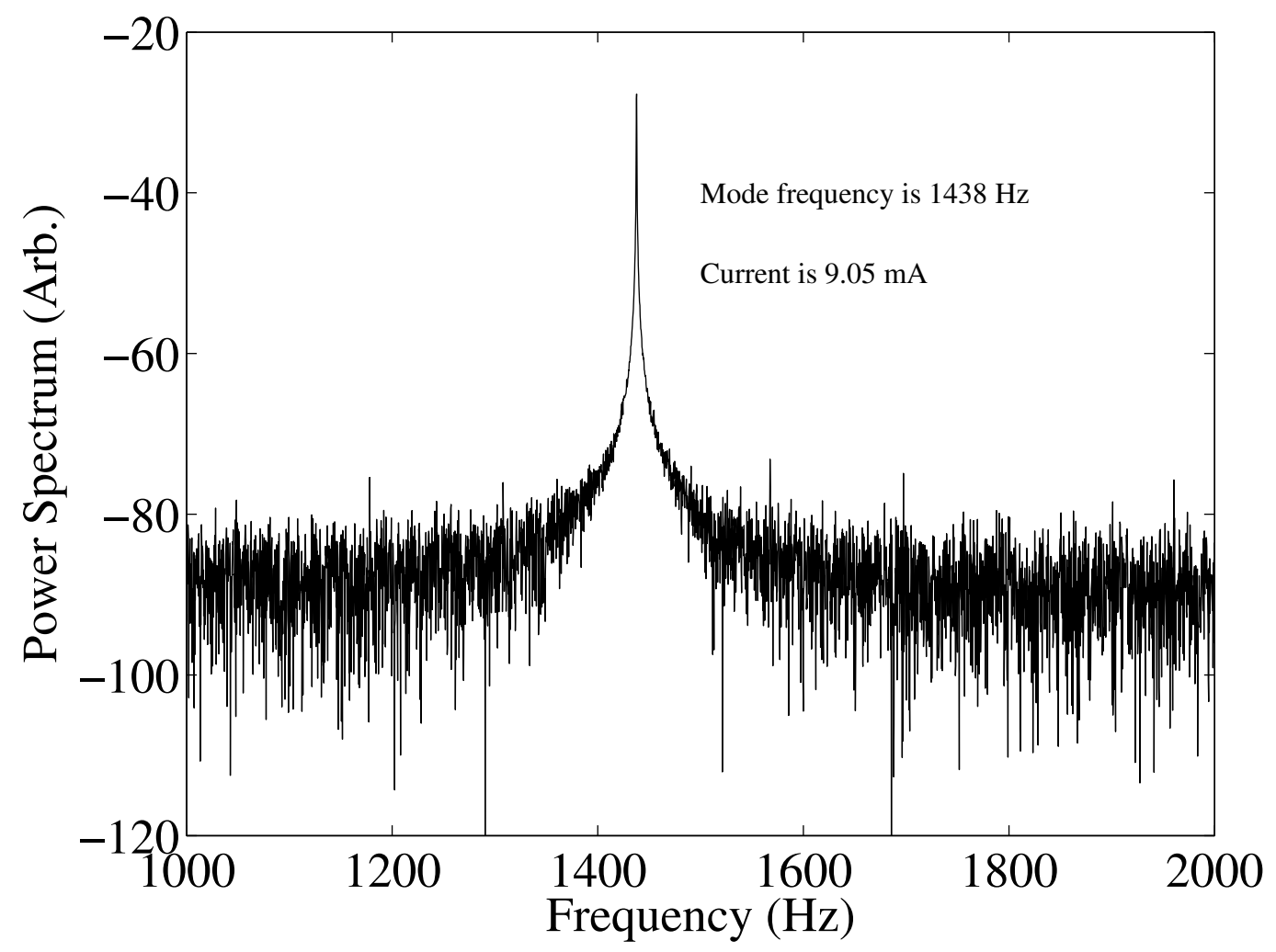

Figure 4.2: An example of the wave spectrum with no driving force. The $y$-axis shows the logarithm of the power spectrum. 

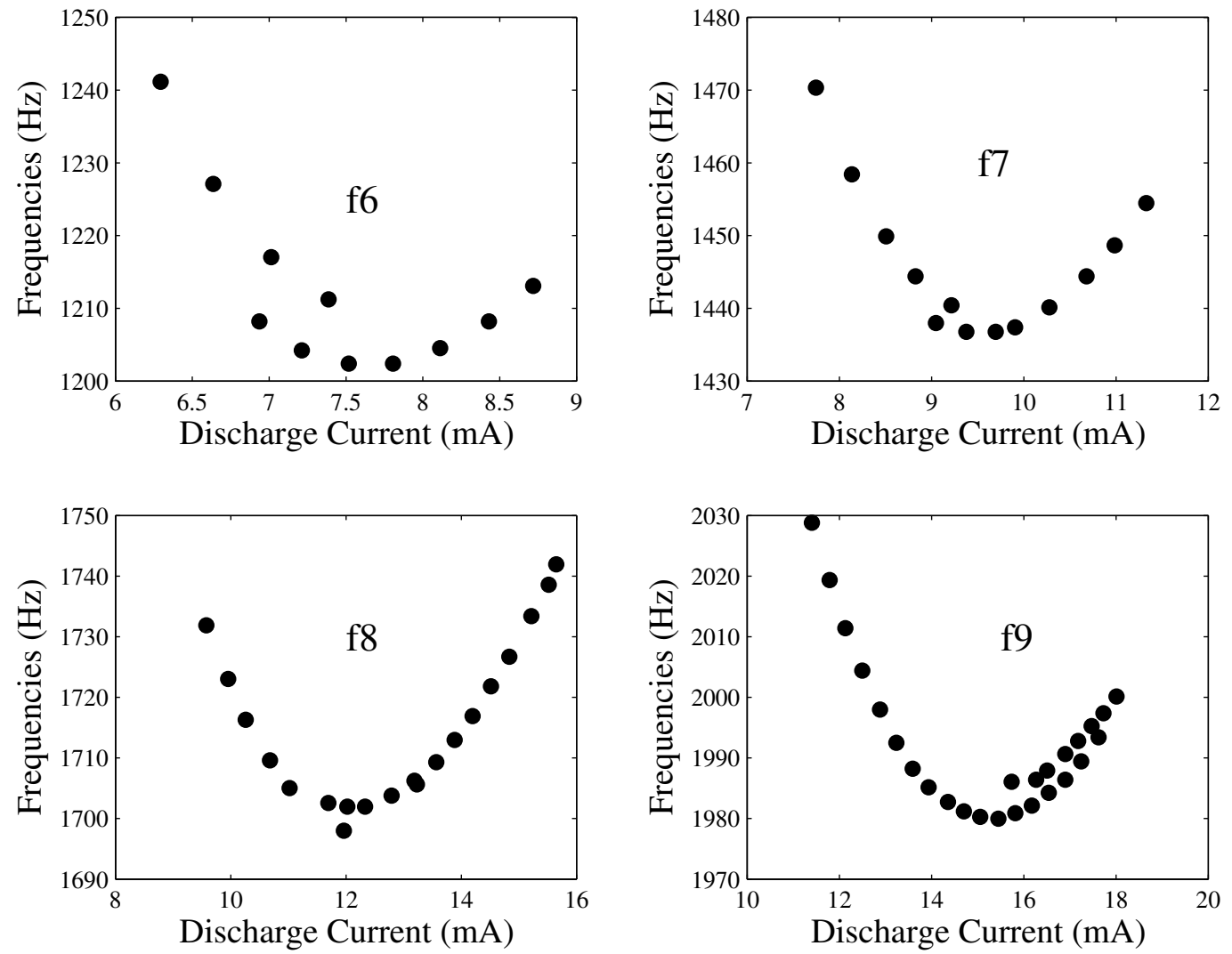

Figure 4.3: Close-up views of four of the wave modes shown in Figure 4.1. Mode frequencies are not constant as discharge current changes. 


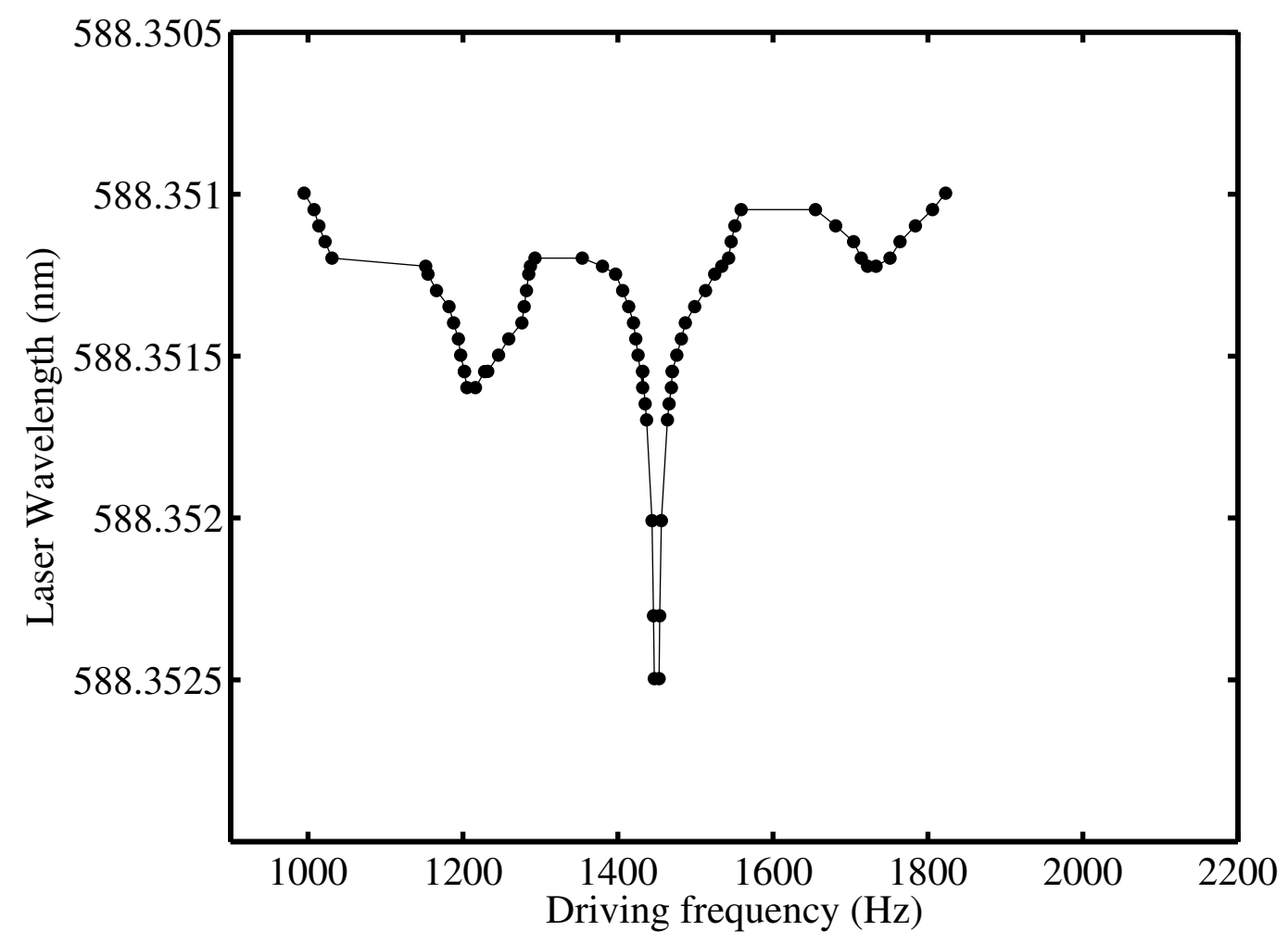

Figure 4.4: Arnold tongue for $I_{D}=9.10 \mathrm{~mA}$. The long feature in the center is the $f 7$ mode. $f 6$ and $f 8$ appear to the left and right, respectively. Since they are not natural modes at this discharge current, $f 6$ and $f 8$ are shorter in the $y$-axis direction. With the laser off, the natural frequency is $\sim 1450 \mathrm{~Hz}$. 


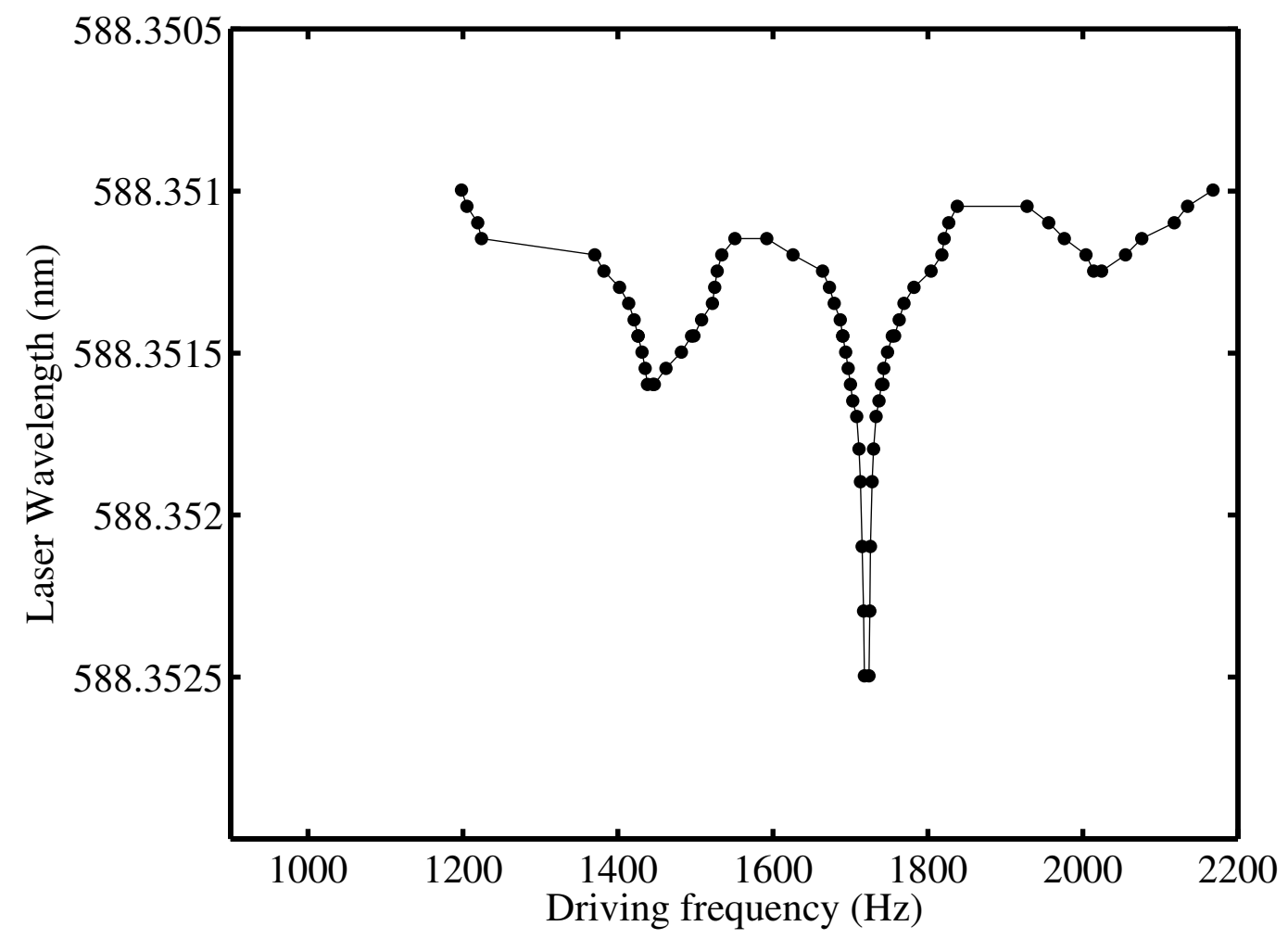

Figure 4.5: Arnold tongue for $I_{D}=11.37 \mathrm{~mA}$. The long feature in the center is the $f 8$ mode. $f 7$ and $f 9$ appear to the left and right, respectively. With the laser off, the natural frequency is $\sim 1721$ $\mathrm{Hz}$. 


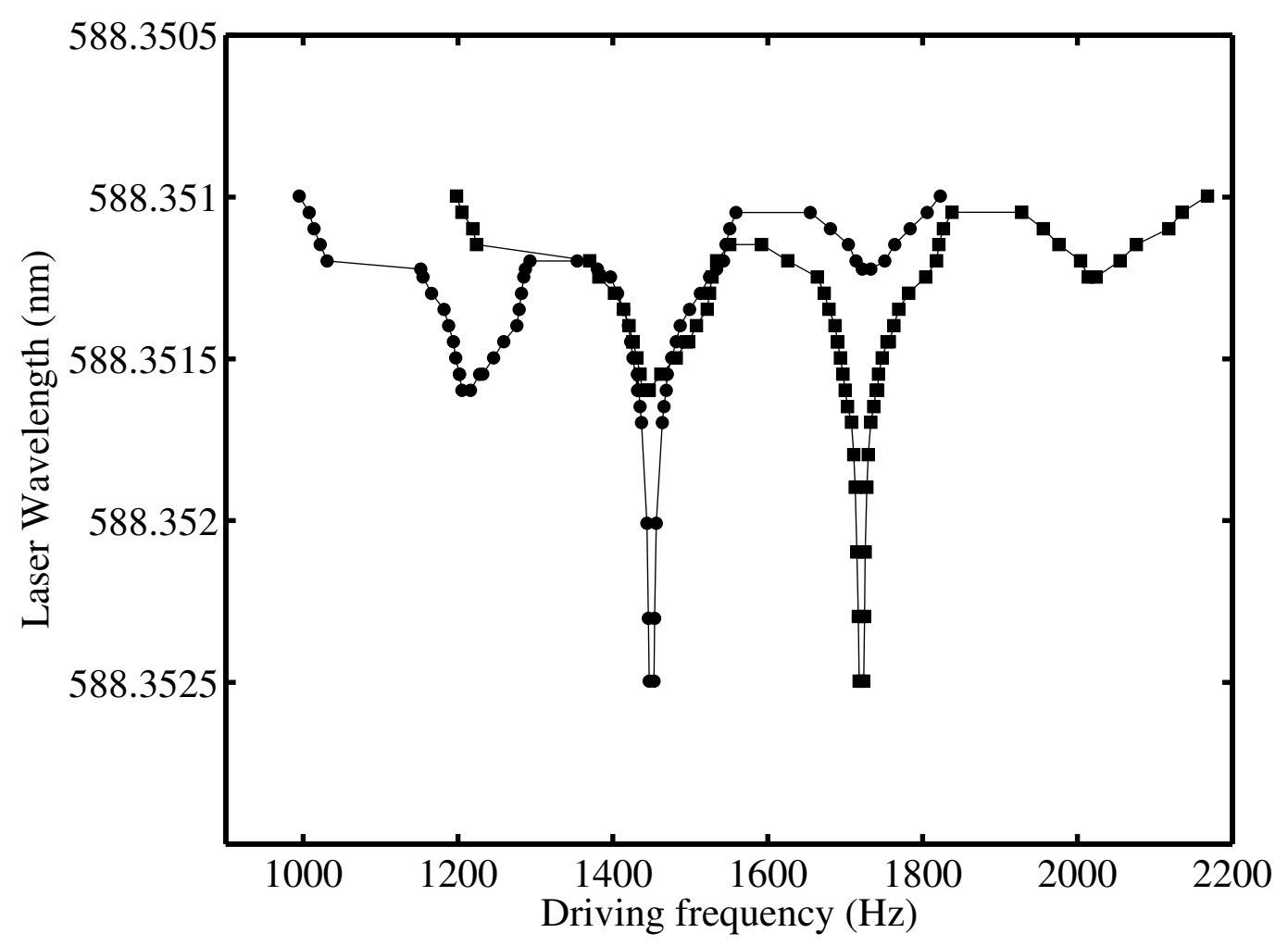

Figure 4.6: Combined view of data from Figures 4.4 and 4.5 showing how the two data sets overlap one another. The currents were $9.10 \mathrm{~mA}(\bullet)$ and $11.37 \mathrm{~mA}(\mathbf{\square})$. 


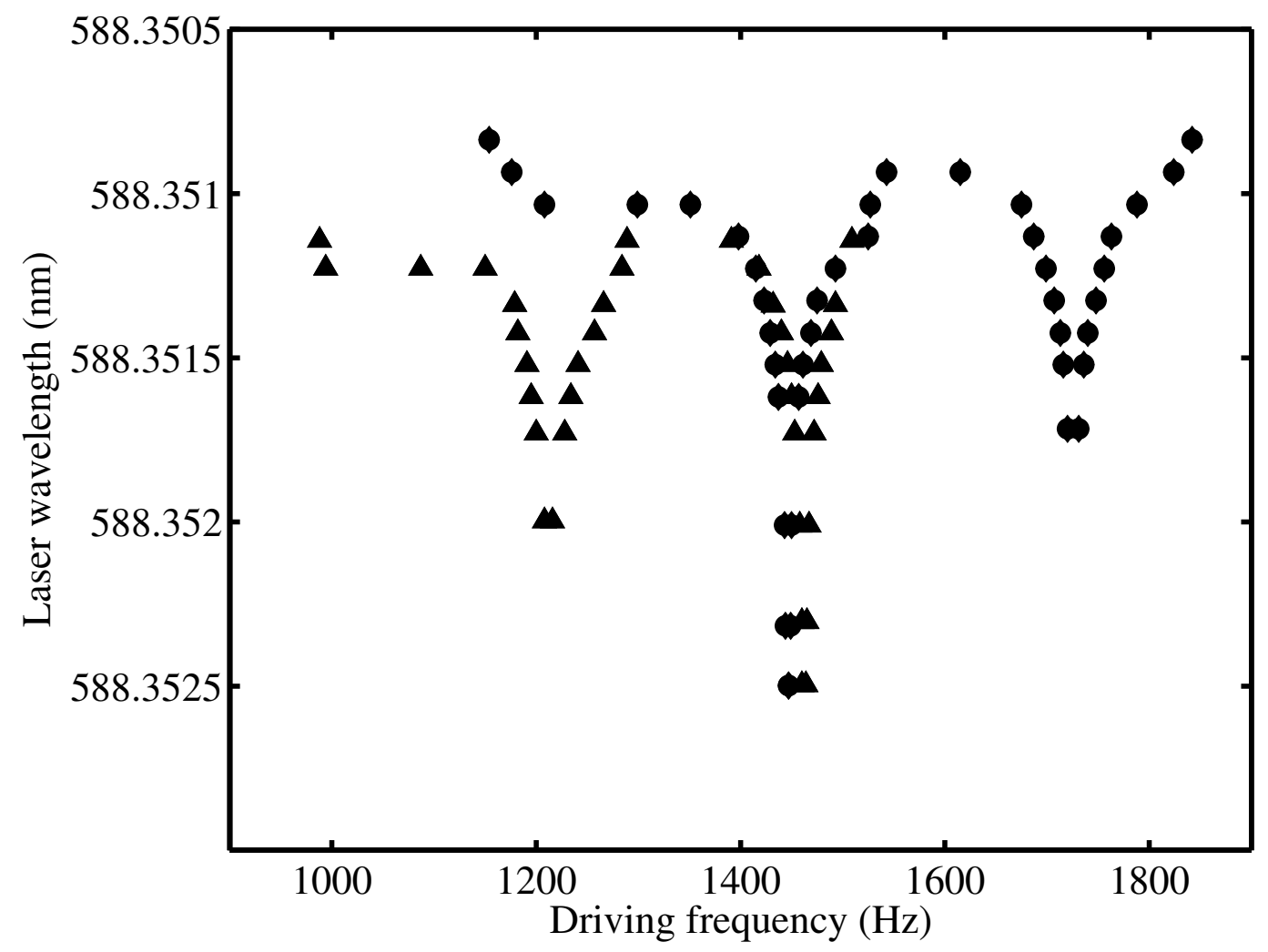

Figure 4.7: Arnol'd tongue boundaries from $I_{D}=8.23 \mathrm{~mA}$ and $I_{D}=10.45 \mathrm{~mA}$, near the mode transition boundaries on either side of the $f 7$ mode. These two boundary maps result from adjusting the discharge current downward $(\boldsymbol{\Lambda})$ or upward $(\bullet)$ from that shown in Figure 4.4. The downward points of the Arnol'd tongue occur at $1212 \mathrm{~Hz}$, at $1462 \mathrm{~Hz}$ (lower current) and at $1447 \mathrm{~Hz}$ (higher current), and $1726 \mathrm{~Hz}$. These values are consistent with Figure 4.3. 


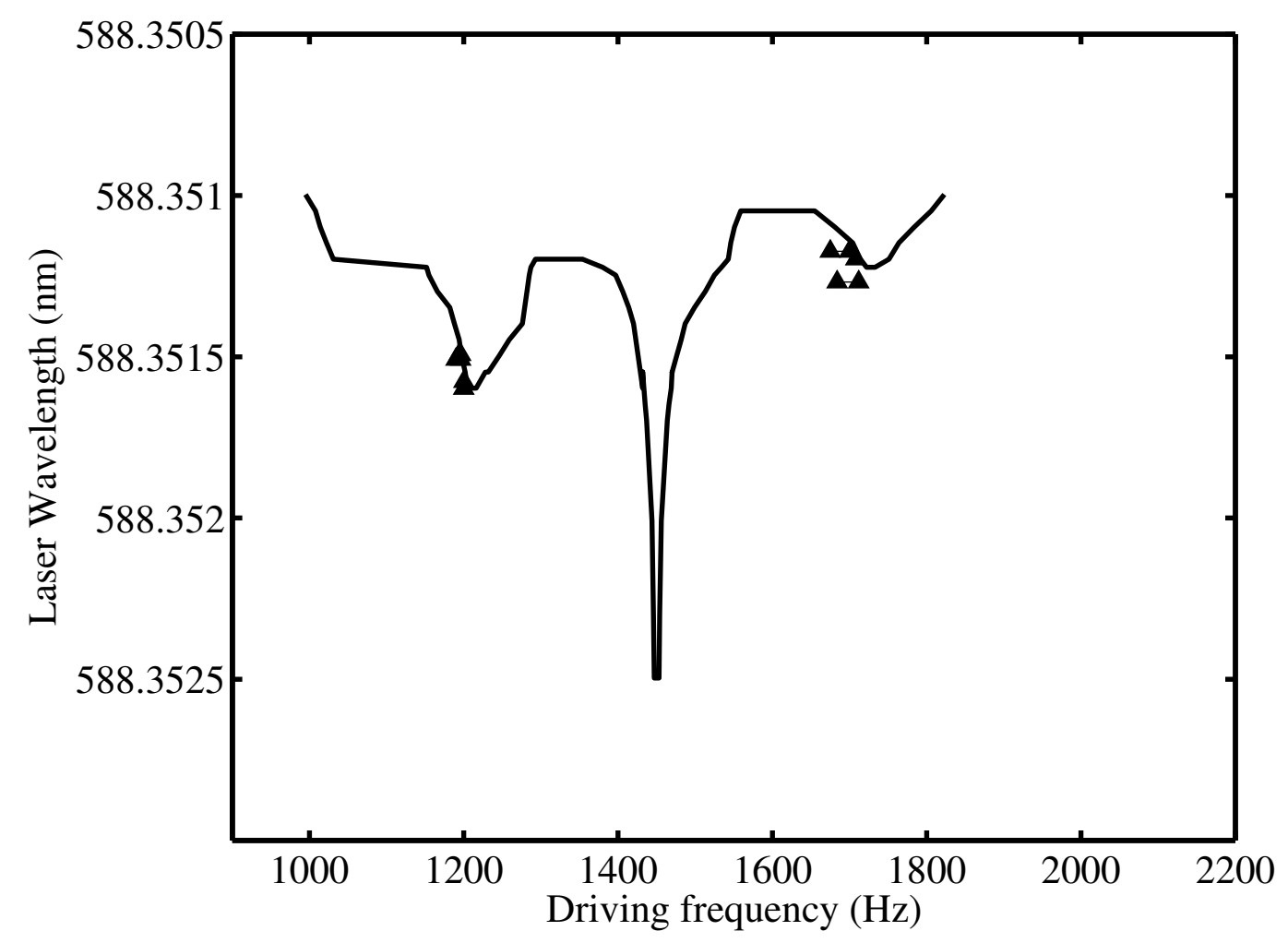

Figure 4.8: The occurrence of dynamics modulation ( $\mathbf{\Delta})$ relative to the Arnol'd tongue of the $f 7$ mode. The cluster of points on the right (left) side result in upward (downward) dynamics modulation. On the right, where two triangles appear side-by-side at the same $y$-axis location, dynamics modulation occurs throughout the interval. Note that all cases of dynamics modulation ended in entrainment, so the small offset between the tip of the $f 8$ mode and the series of triangles is not significant. 


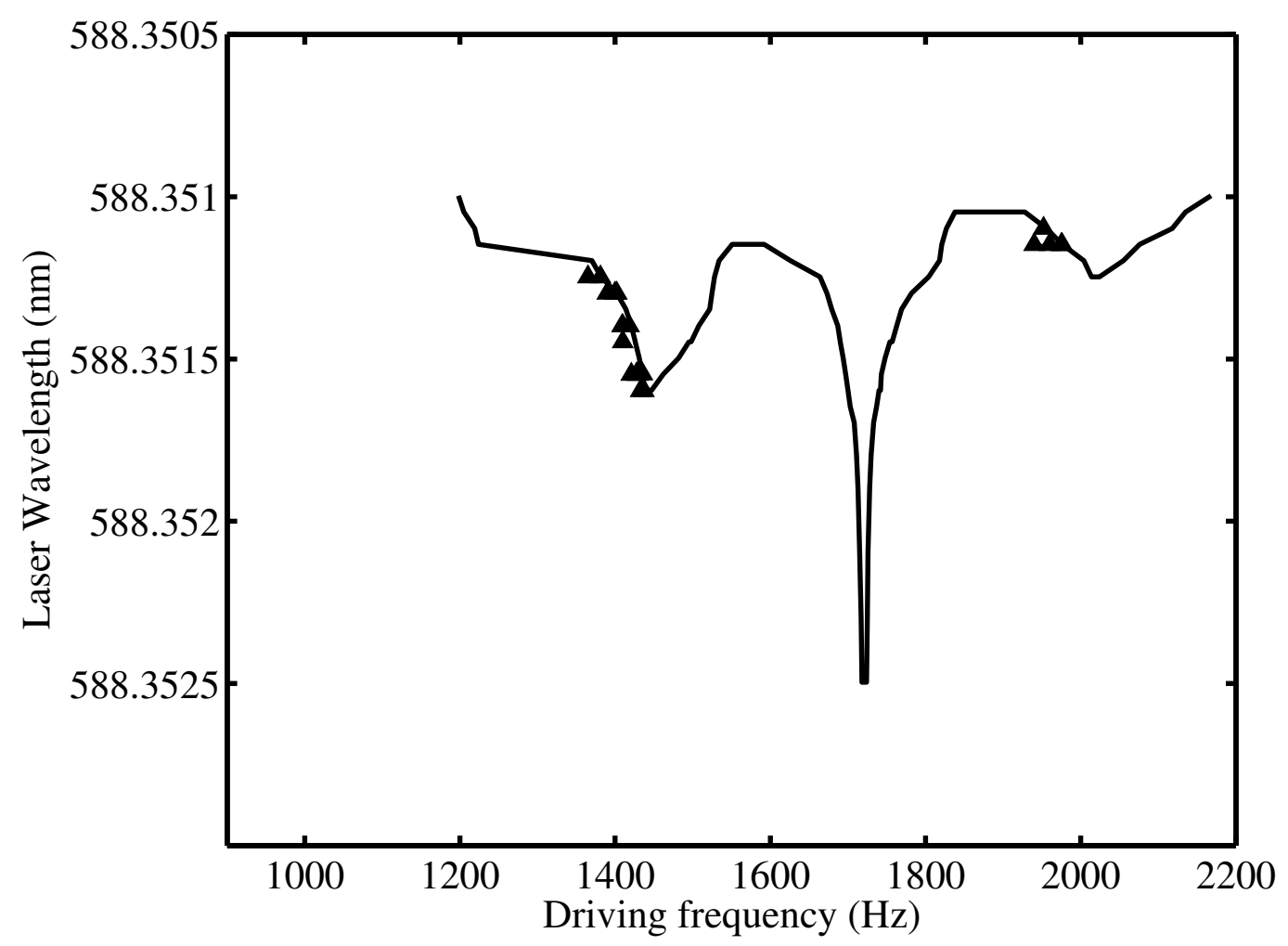

Figure 4.9: The occurrence of dynamics modulation ( $\mathbf{\Delta})$ relative to the Arnol'd tongue of the f8 mode. The cluster of points on the right (left) side result in upward (downward) dynamics modulation. On the right, where two triangles appear side-by-side at the same $y$-axis location, dynamics modulation occurs throughout the interval. 


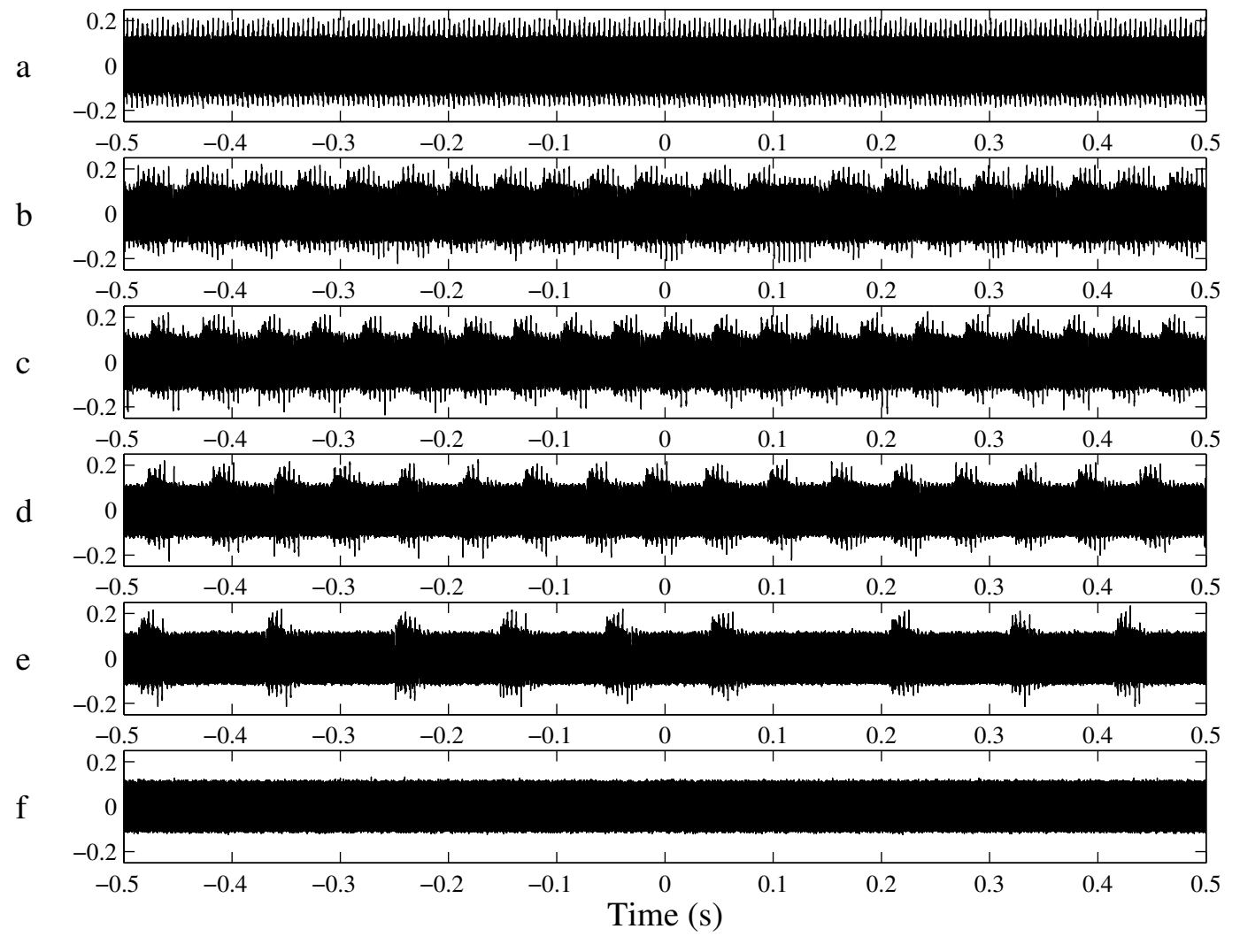

Figure 4.10: A sequence of time series for upward dynamics modulation as a function of driving frequency. The $y$-axis of each plot shows the signal amplitude from the photodiode. Beginning at the top, the driving frequency $\left(f_{i}\right)$ is 1681 (a), 1688 (b), 1696 (c), 1704 (d), 1710 (e) and 1713 (f) $\mathrm{Hz}$. The discharge current was set to $9.10 \mathrm{~mA}$. Entrainment occurred at $1712 \mathrm{~Hz}$. 


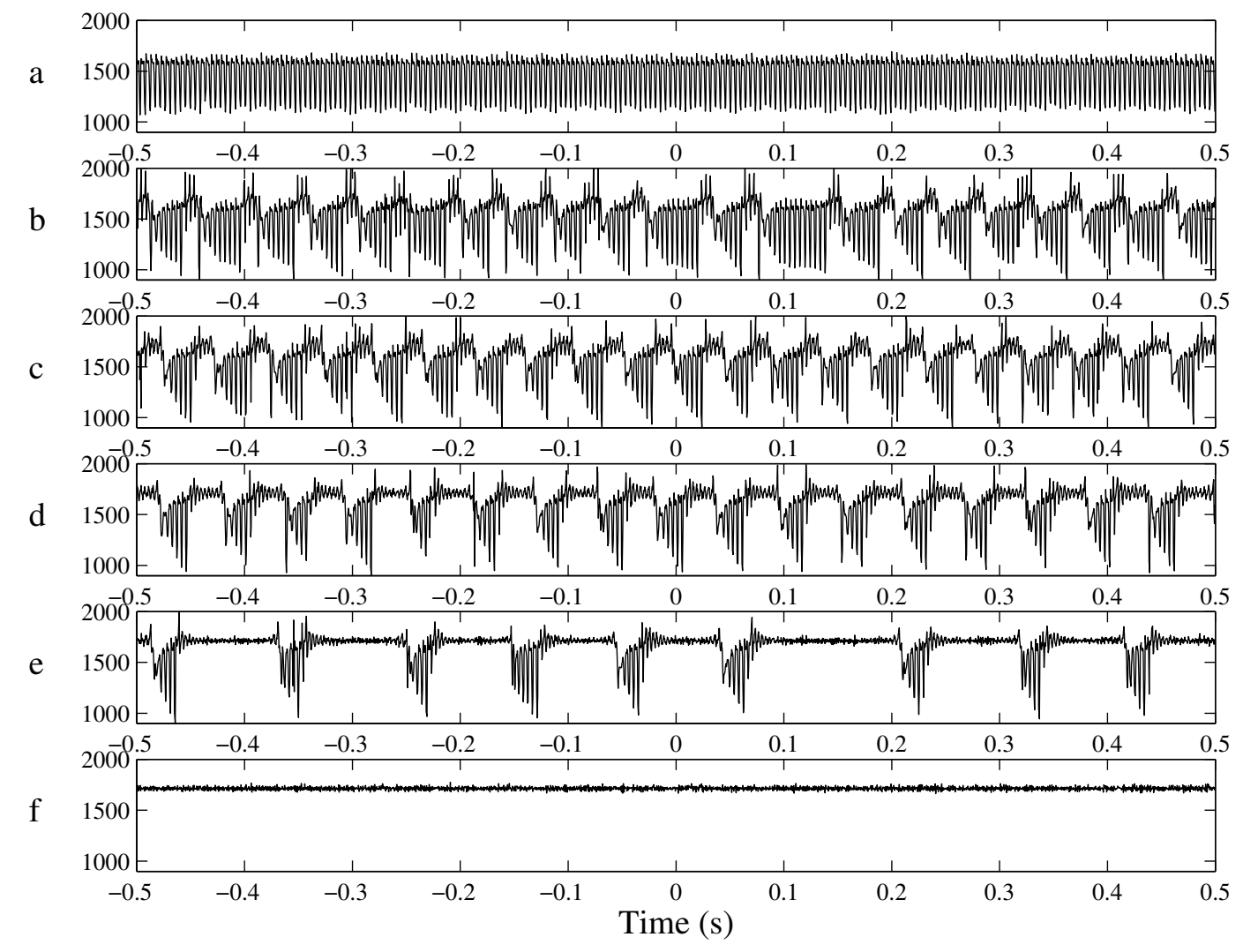

Figure 4.11: A sequence of instantaneous frequency series for upward dynamics modulation as a function of driving frequency. The $y$-axis of each plot shows the instantaneous frequency in $\mathrm{Hz}$ for the time series shown in Figure 4.10. Beginning at the top, the driving frequency $\left(f_{i}\right)$ is 1681 (a), 1688 (b), 1696 (c), 1704 (d), 1710 (e) and 1713 (f) Hz. The modulation is taking place between the $f 7$ and $f 8$ modes. The discharge current was set to $9.10 \mathrm{~mA}$. Entrainment occurred at $1712 \mathrm{~Hz}$. 
a

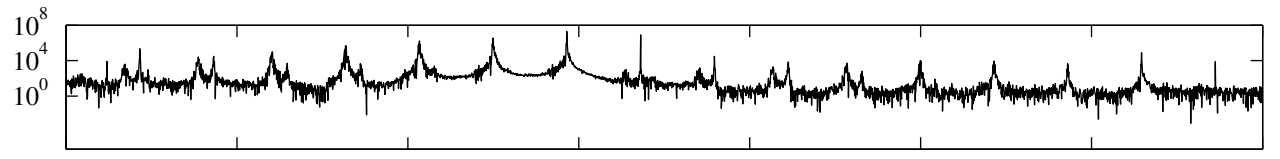

b

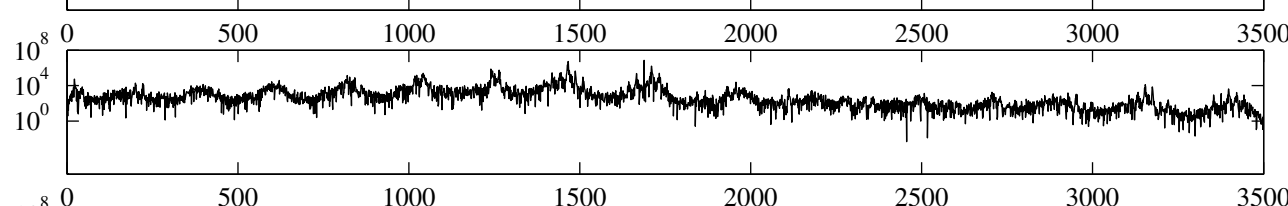

$\mathrm{c}$

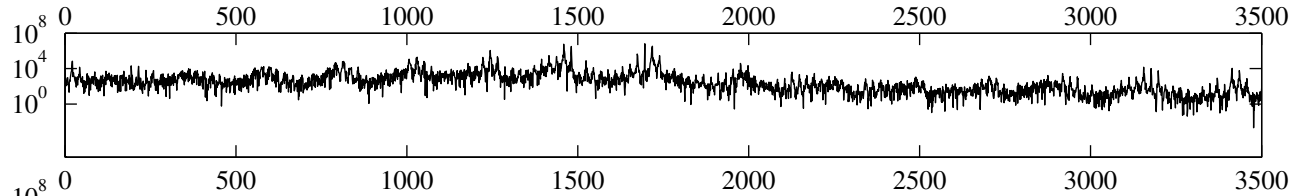

d

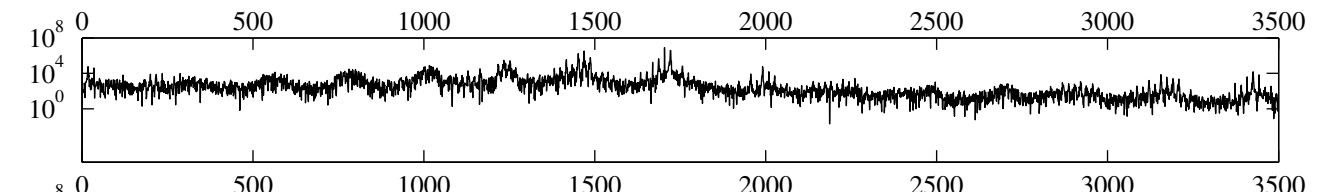

$\mathrm{e}$

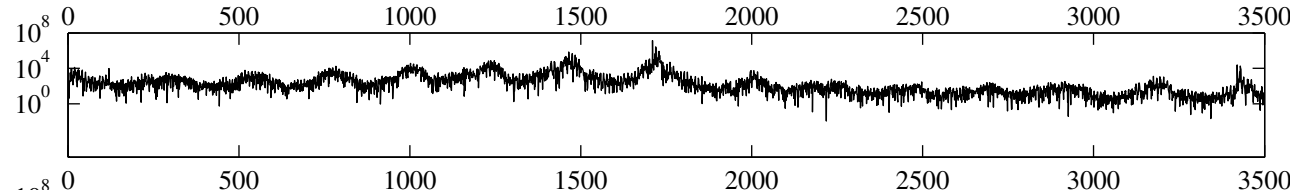

f

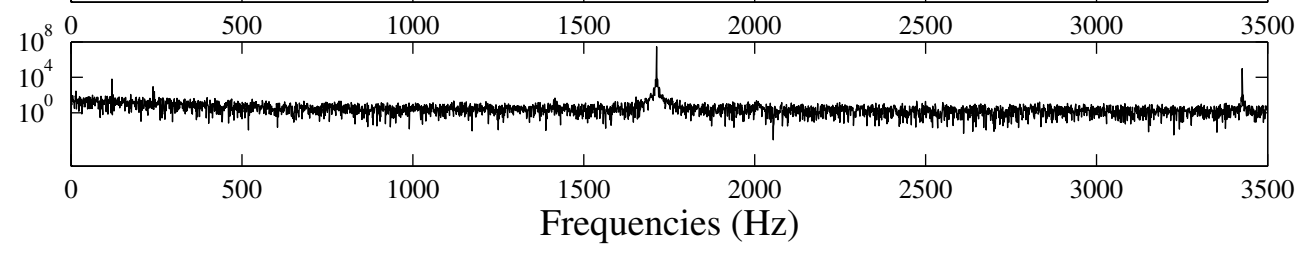

Figure 4.12: A sequence of power spectra for upward dynamics modulation as a function of driving frequency. The power spectra corresponding to the time series shown in Figure 4.10. Beginning at the top, the driving frequency $\left(f_{i}\right)$ is 1681 (a), 1688 (b), 1696 (c), 1704 (d), 1710 (e) and 1713 (f) $\mathrm{Hz}$. The discharge current was set to $9.10 \mathrm{~mA}$. Entrainment occurred at $1712 \mathrm{~Hz}$. 

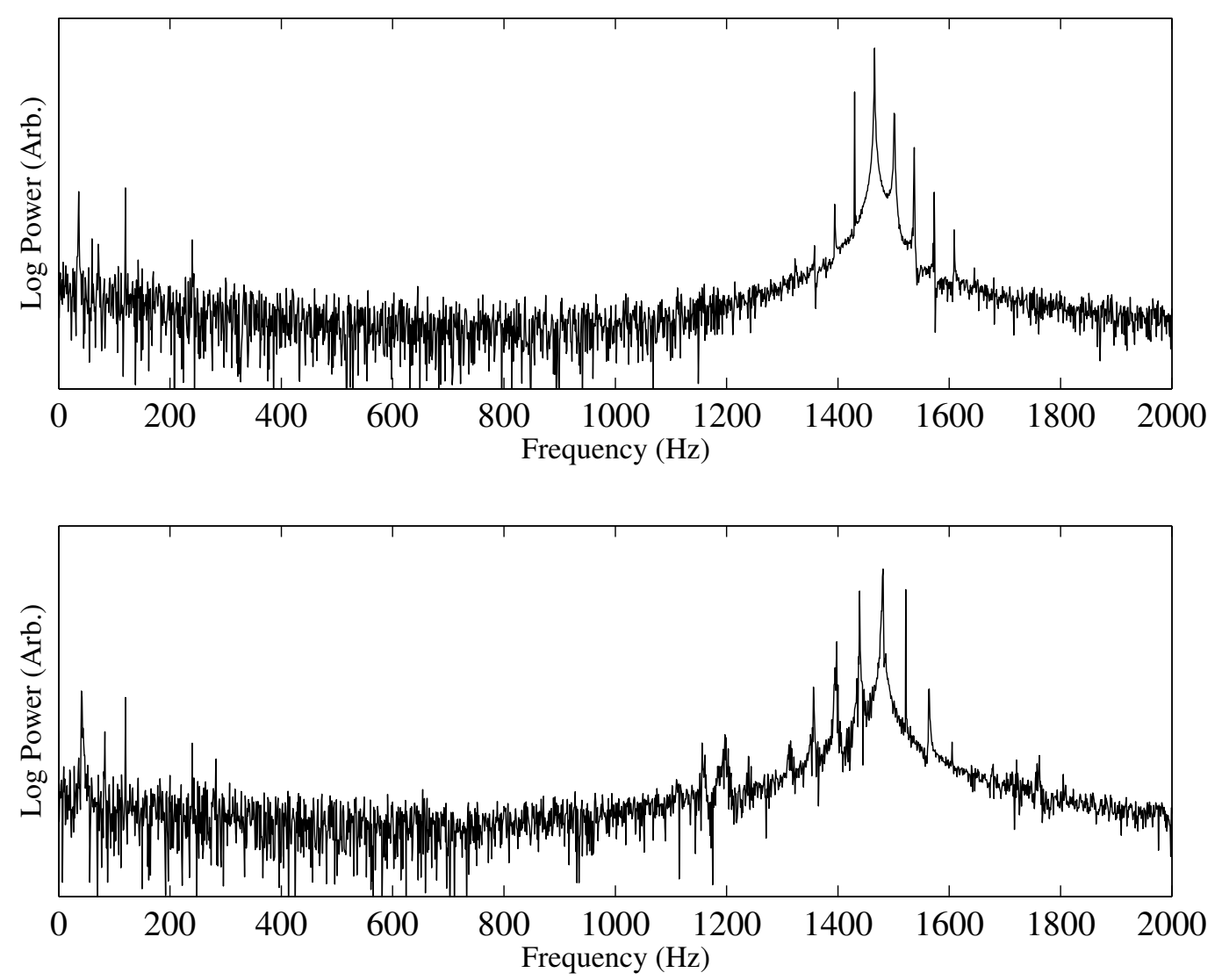

Figure 4.13: A wider view, including low frequencies, of the two spectra shown in Figure 3.7. There is a dominant low frequency peak that corresponds to the spacing between the sidebands on the main feature. This feature can be used to identify the modified beat frequency $\Omega$, which is then used to find the entrainment parameter $\alpha$ for temporal periodic pulling when the method described in Chapter III is obscured by the activity of dynamics modulation. 

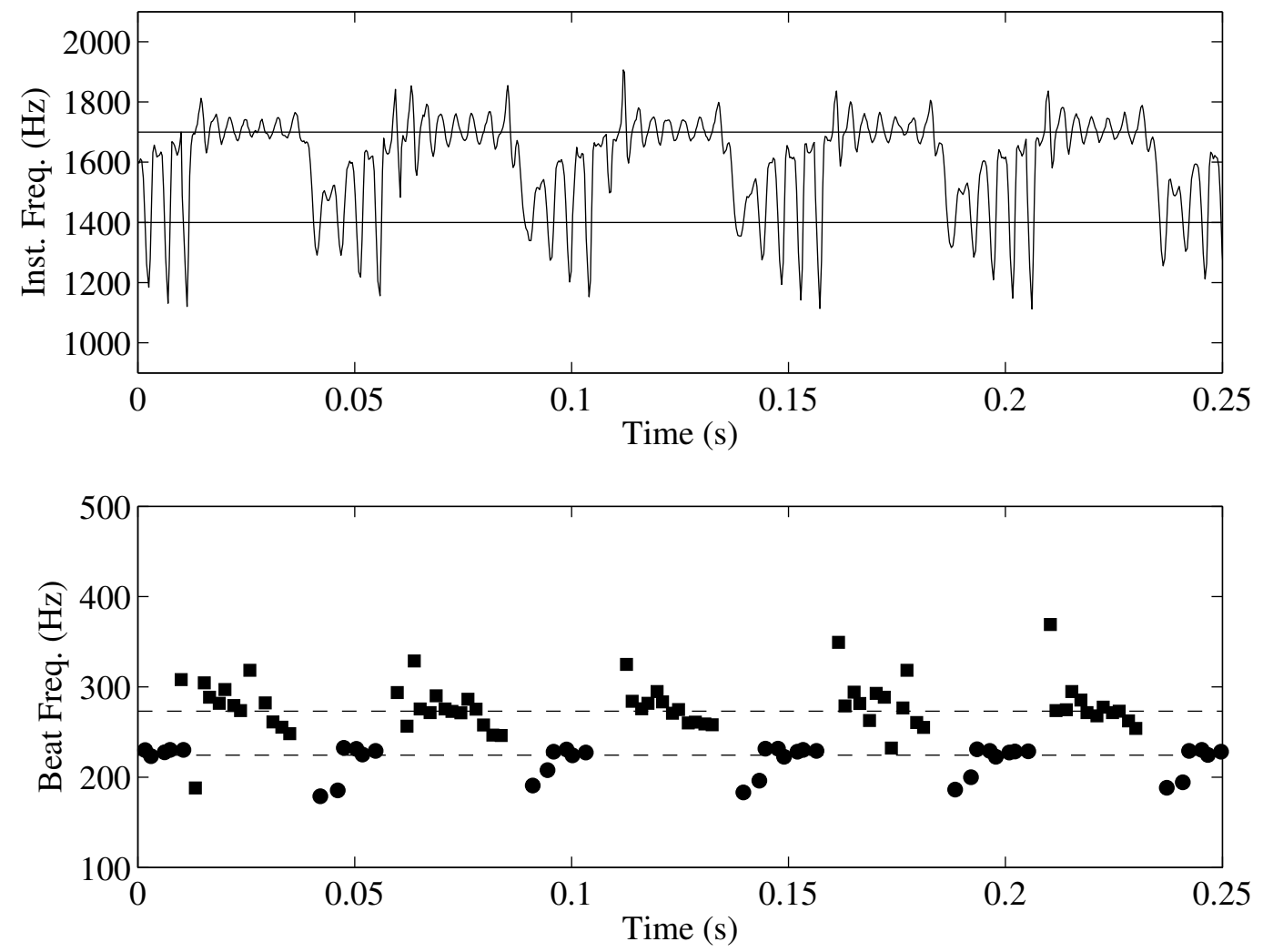

Figure 4.14: An example that shows how to calculate the $\Omega$ frequency for the two states of dynamics modulation. The top panel shows an instantaneous frequency series, with horizontal lines drawn at 1350 and $1700 \mathrm{~Hz}\left(=f_{i}\right)$. The beat frequency was calculated using the instantaneous frequency routine on this series. Instead of examining zero crossings, crossings of the two lines were used. The beat frequency modulated between two values, as can be seen in the lower panel. The dashed lines represent the median values of the lower $(\bullet, 224.3 \mathrm{~Hz})$ and higher $(\boldsymbol{\square}, 273.1 \mathrm{~Hz}) \mathrm{measured}$ beats. The discharge current was set to $9.10 \mathrm{~mA}$. Entrainment occurred at $1713 \mathrm{~Hz}$. 

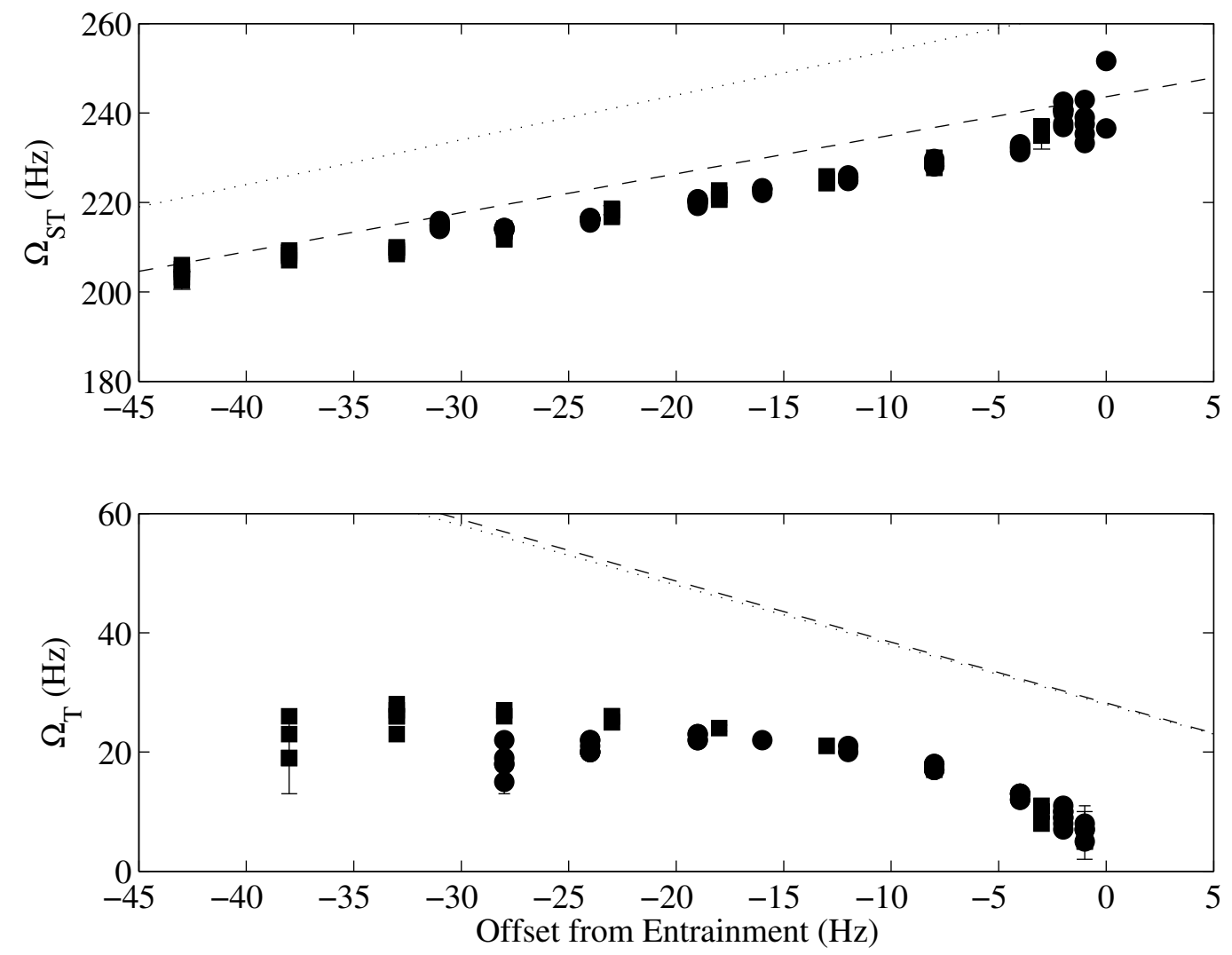

Figure 4.15: Reproducible values for $\Omega_{S T}$ and $\Omega_{T}$ obtained from dynamics modulation data sets. These come from the sequence of time series highlighted in Figures 4.10 through 4.12, as well as a second series taken on a different day, depicting upward modulation between $f 7$ and $f 8$. In the top (bottom) panel, the spatiotemporal (temporal) beat frequencies are calculated for two data sets on two different days, as a function of entrainment frequency minus driving frequency. The dotted line shows the conventional beat frequency $\left(\Omega_{0}\right)$ and the dashed line shows the reference beat frequency $\left(\Omega^{\prime}\right)$. 

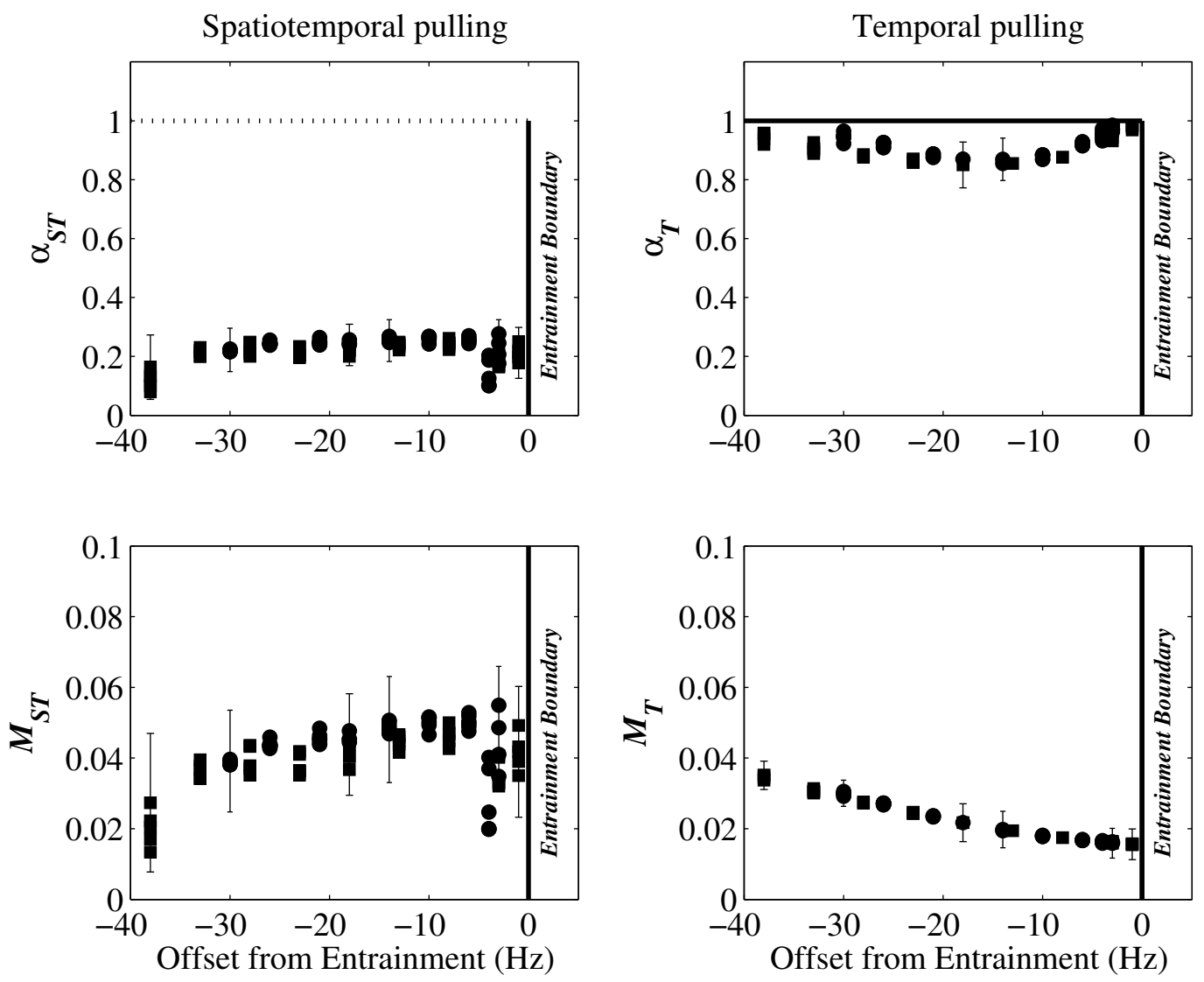

Figure 4.16: The results of $\alpha$ and $M$ calculations from the sequence of time series highlighted in Figures 4.10 through 4.12, as well as a second series taken on a different day, depicting upward modulation between $f 7$ and $f 8$. On the left is the spatiotemporal case in which the entrained upper mode drives the lower mode. On the right, the temporal case in which the driver drives the upper mode. The $x$-axis displays entrainment frequency minus driving frequency. The solid lines to the right (and near the top of the temporal $\alpha$ subplot) indicate the entrainment boundary for the $f 8$ mode. The dotted line in the top-left panel indicates the entrainment boundary for the $f 7$ mode, which is never approached. Note that $\alpha_{T}$ approaches one just as the upper mode becomes entrained. The discharge current was set to $9.10 \mathrm{~mA}$. 

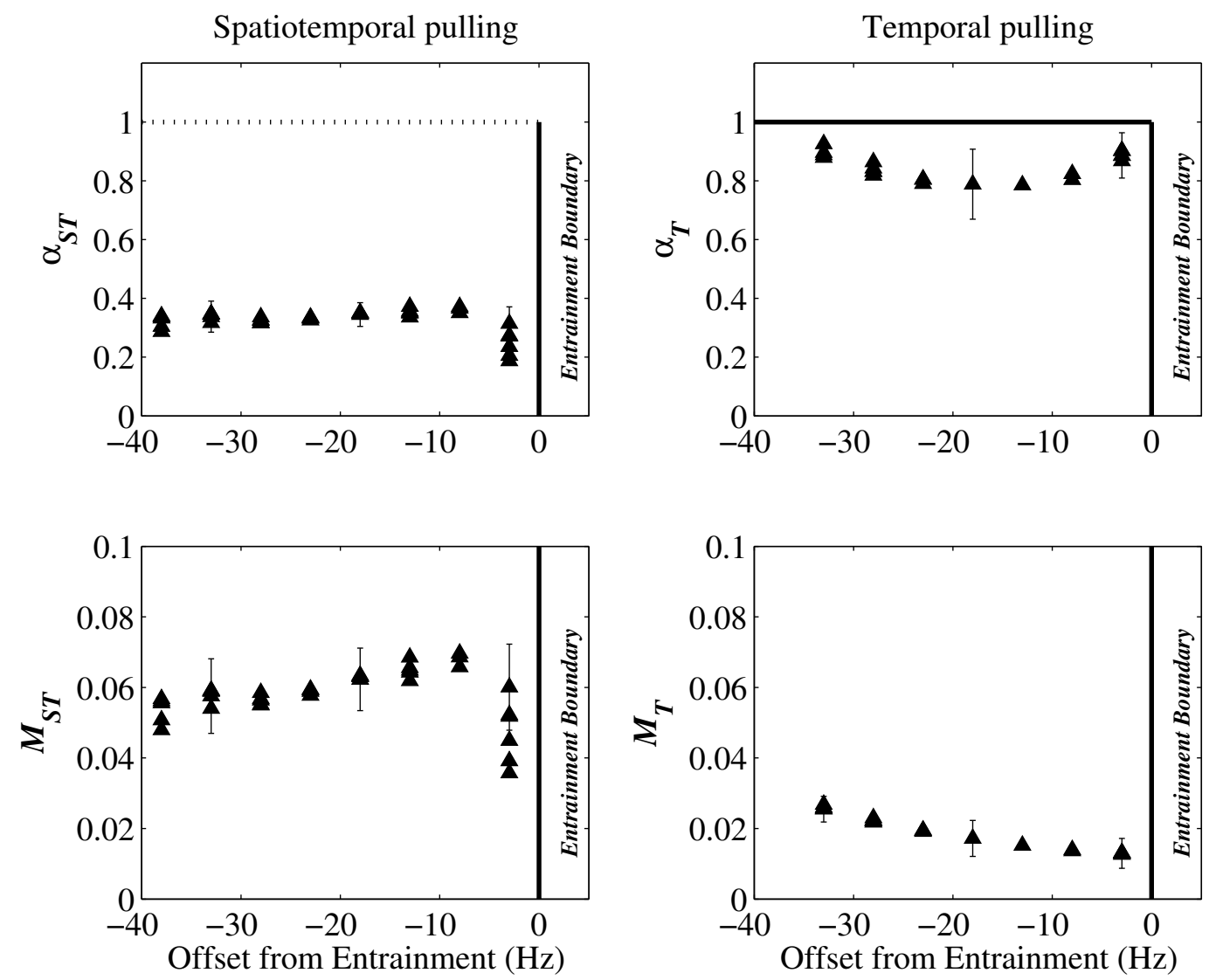

Figure 4.17: The results of $\alpha$ and $M$ calculations from another sequence of time series taken on a different day and at a different discharge current. In this case the data depicts upward modulation between $f 8$ and $f 9$. Again, on the left is the spatiotemporal case in which the entrained upper mode drives the lower mode. On the right, the temporal case in which the driver drives the upper mode. The solid lines to the right (and near the top of the temporal $\alpha$ subplot) indicate the entrainment boundary for the $f 9$ mode. The dotted line in the top-left panel indicates the entrainment boundary for the $f 8$ mode, which is never approached. Again, $\alpha_{T}$ approaches one just as the upper mode becomes entrained, though not quite as convincingly. The discharge current was set to $11.37 \mathrm{~mA}$. Entrainment occurred at $2013 \mathrm{~Hz}$. 


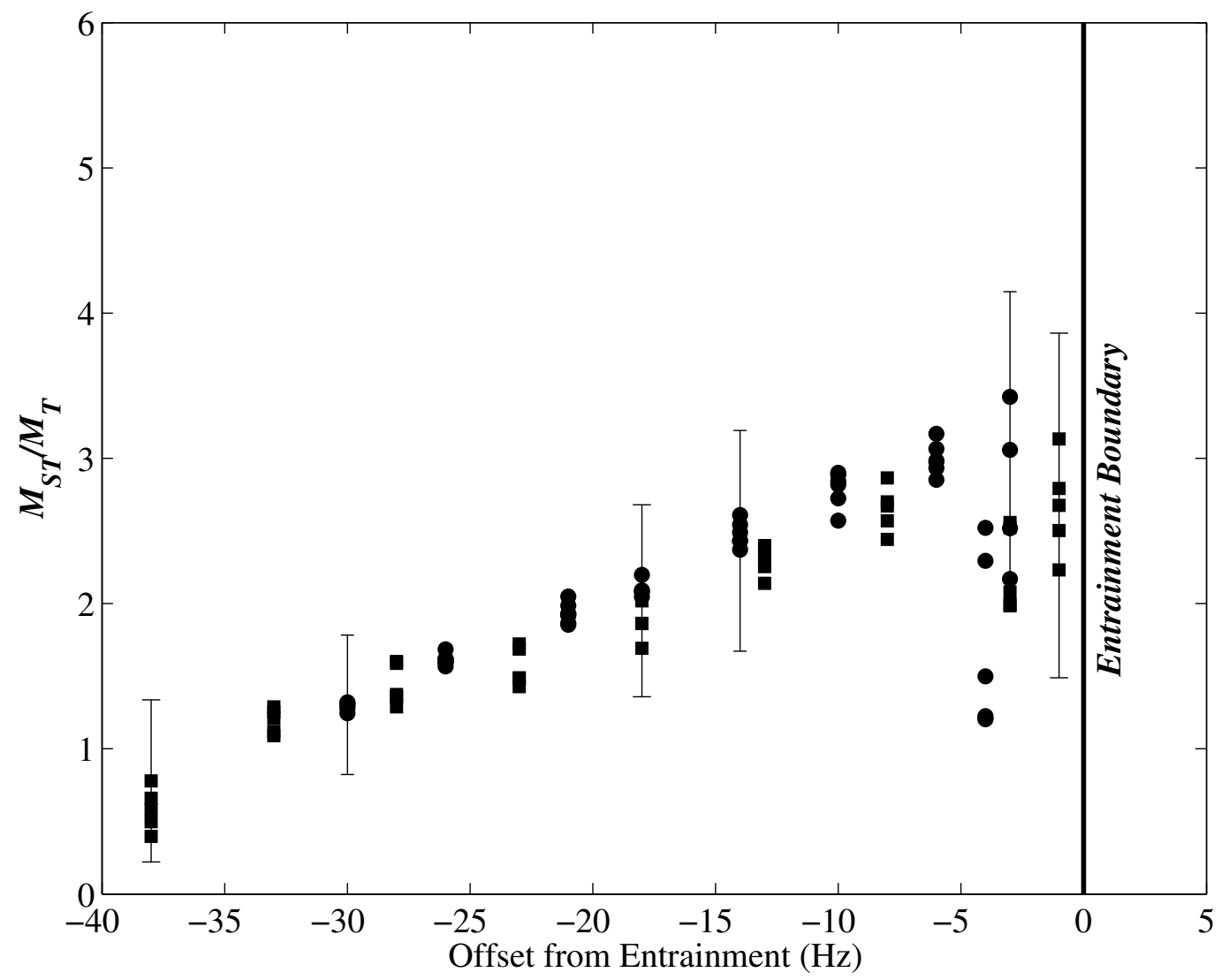

Figure 4.18: The ratio of $M_{S T}$ to $M_{T}$ for the two data sets of Figure 4.16. The ratio steadily increases as the entrainment boundary for the $f 8$ mode is approached. The discharge current was set to $9.10 \mathrm{~mA}$. Entrainment occurred at $1712(\bullet)$ and $1713(\boldsymbol{\square}) \mathrm{Hz}$ on the two days, with the difference being due to slight variation in laser power and/or position. 


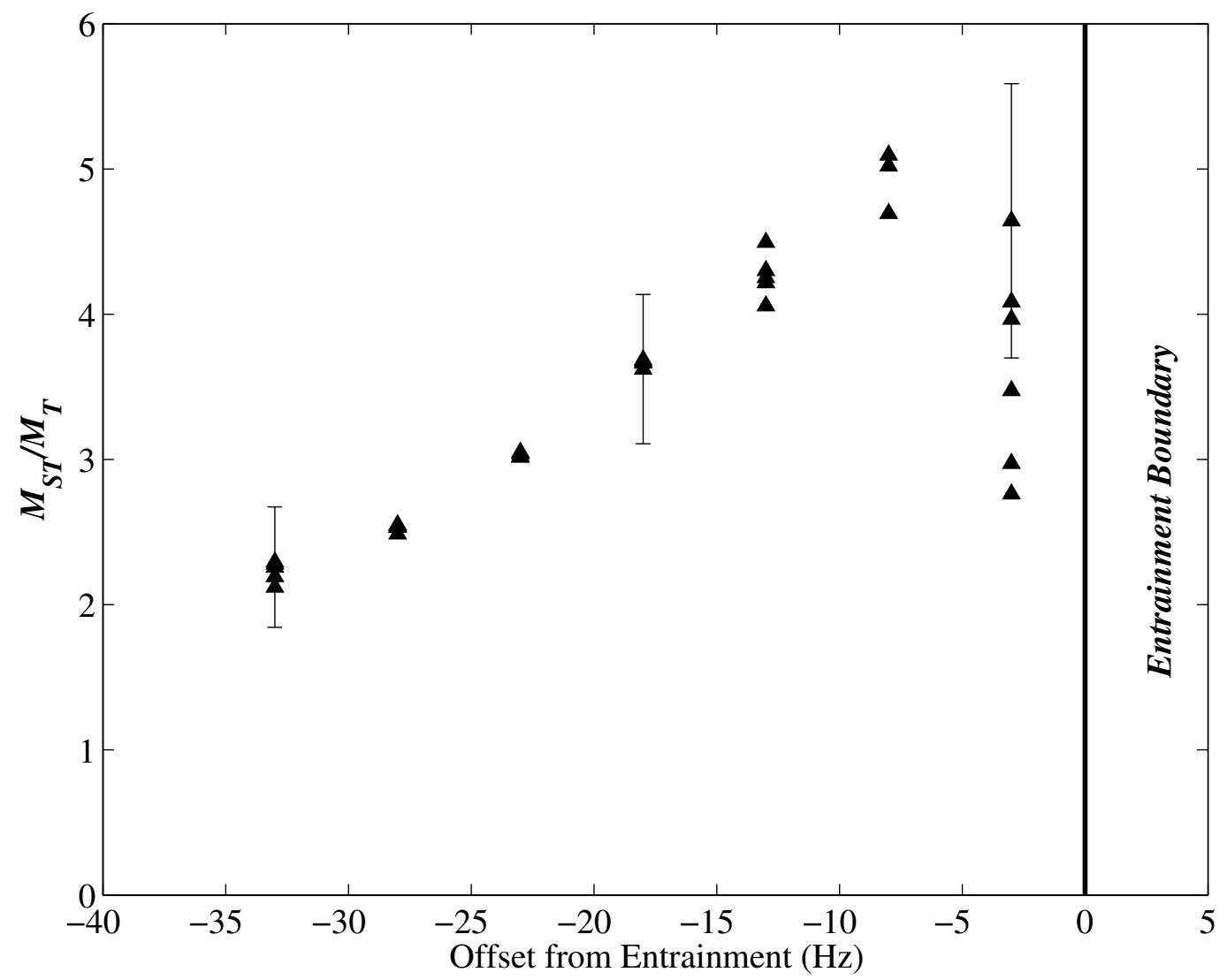

Figure 4.19: The ratio of $M_{S T}$ to $M_{T}$ for the data of Figure 4.17. Again, the ratio steadily increases as entrainment boundary for the $f 9$ mode is approached. The discharge current was set to 11.37 mA. Entrainment occurred at $2013 \mathrm{~Hz}$. 

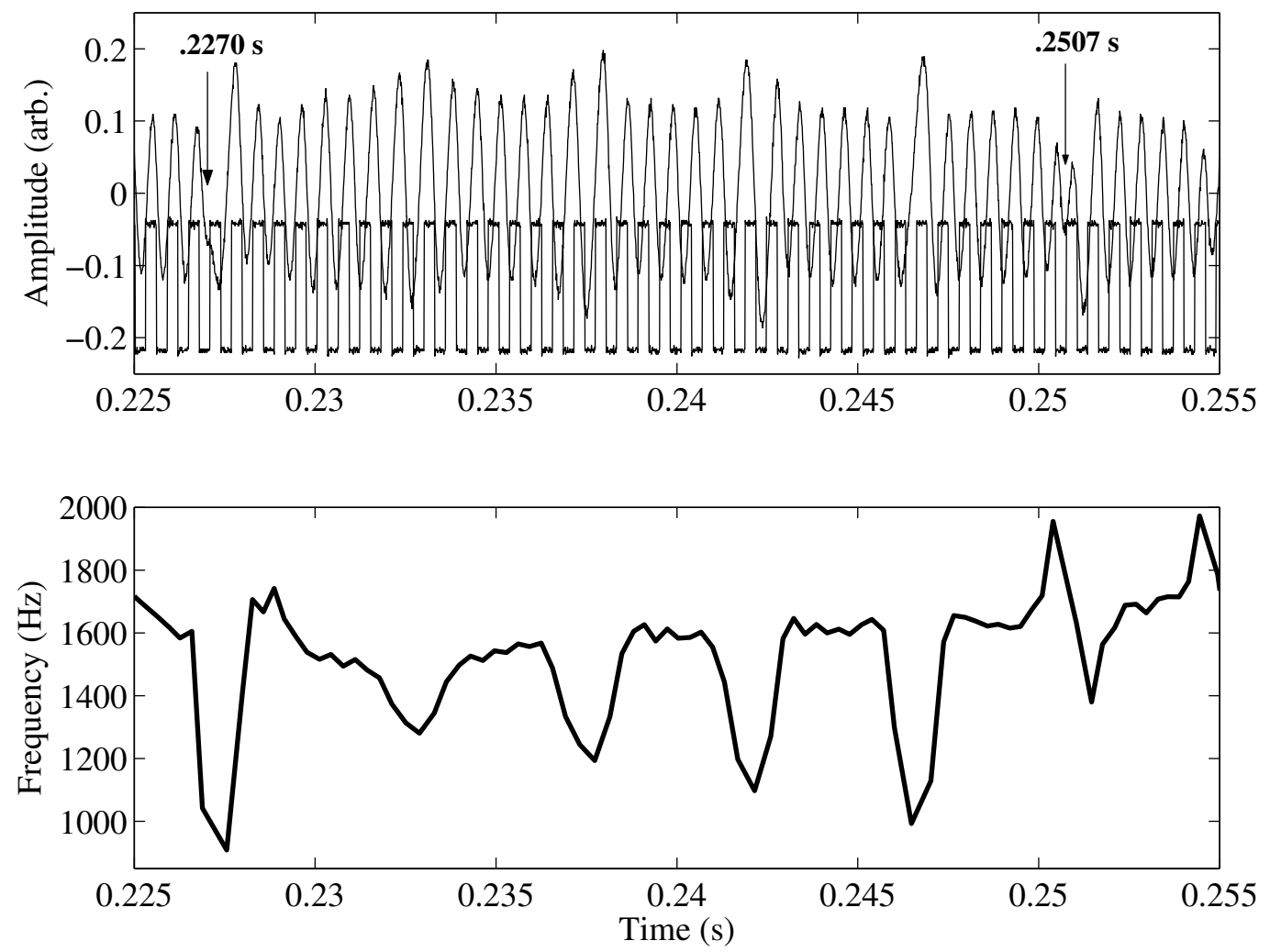

Figure 4.20: Time series close-up of lower mode during periodic dynamics modulation. Along with the upper, time series trace, the square-wave driving frequency is shown scaled and offset. This segment of the modulation begins with a discontinuity in the time series at $t=0.2270 \mathrm{~s}$, and ends at $t=0.2507 \mathrm{~s}$. During the time spent in the lower mode, the amplitude beats with a period of $0.00457 \mathrm{~s}$. The driving frequency, $f_{i}$, was set to $1690 \mathrm{~Hz}$. 

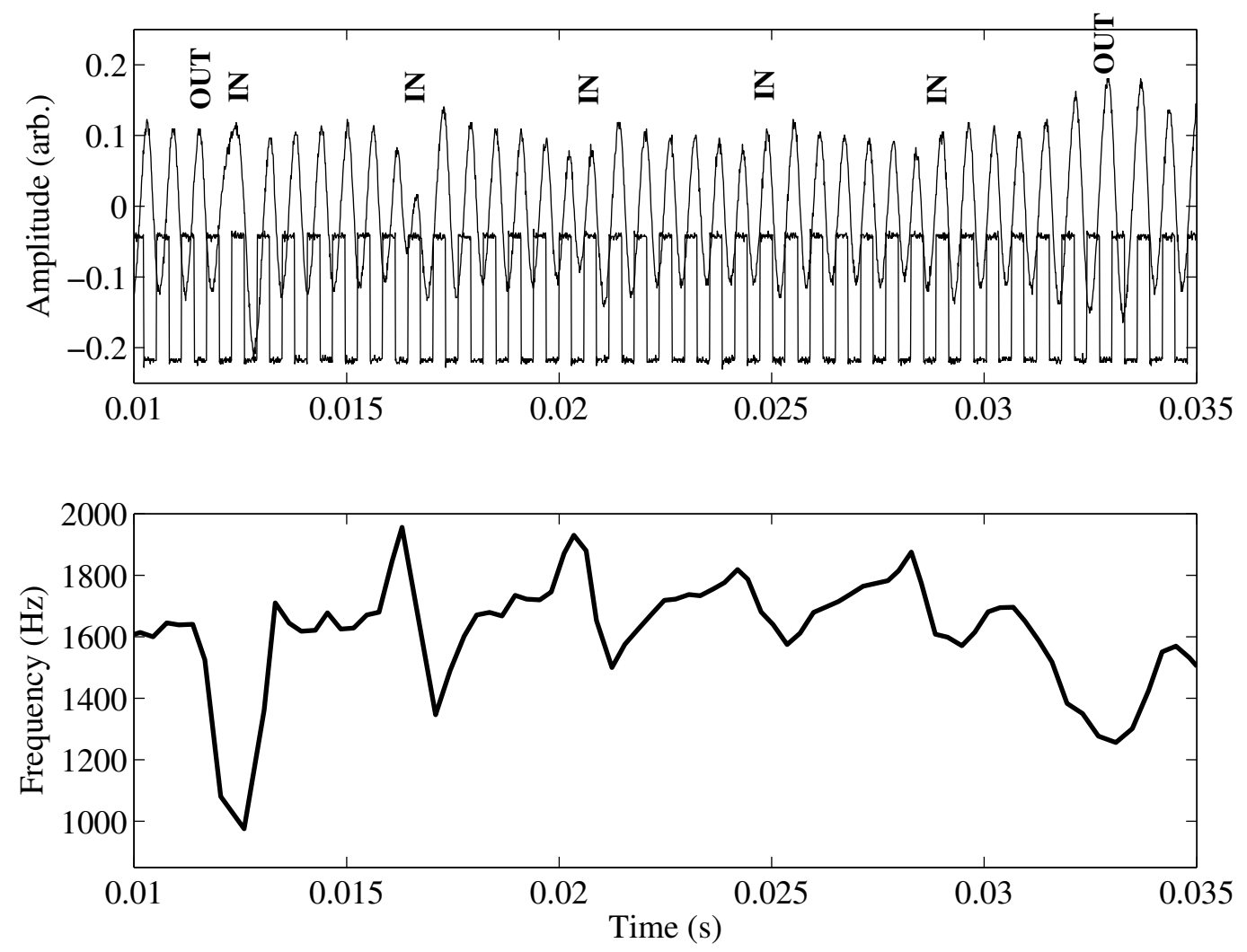

Figure 4.21: Time series close-up of higher mode during periodic dynamics modulation. This segment of the modulation begins when the time series and driver quickly change from out-ofphase to in-phase at $t=0.0124 \mathrm{~s}$, and ends with the opposite change at $t=0.0329 \mathrm{~s}$. During the time spent in the higher mode, the amplitude beats with a period of $0.00430 \mathrm{~s}$. The driving frequency, $f_{i}$, was set to $1690 \mathrm{~Hz}$. 

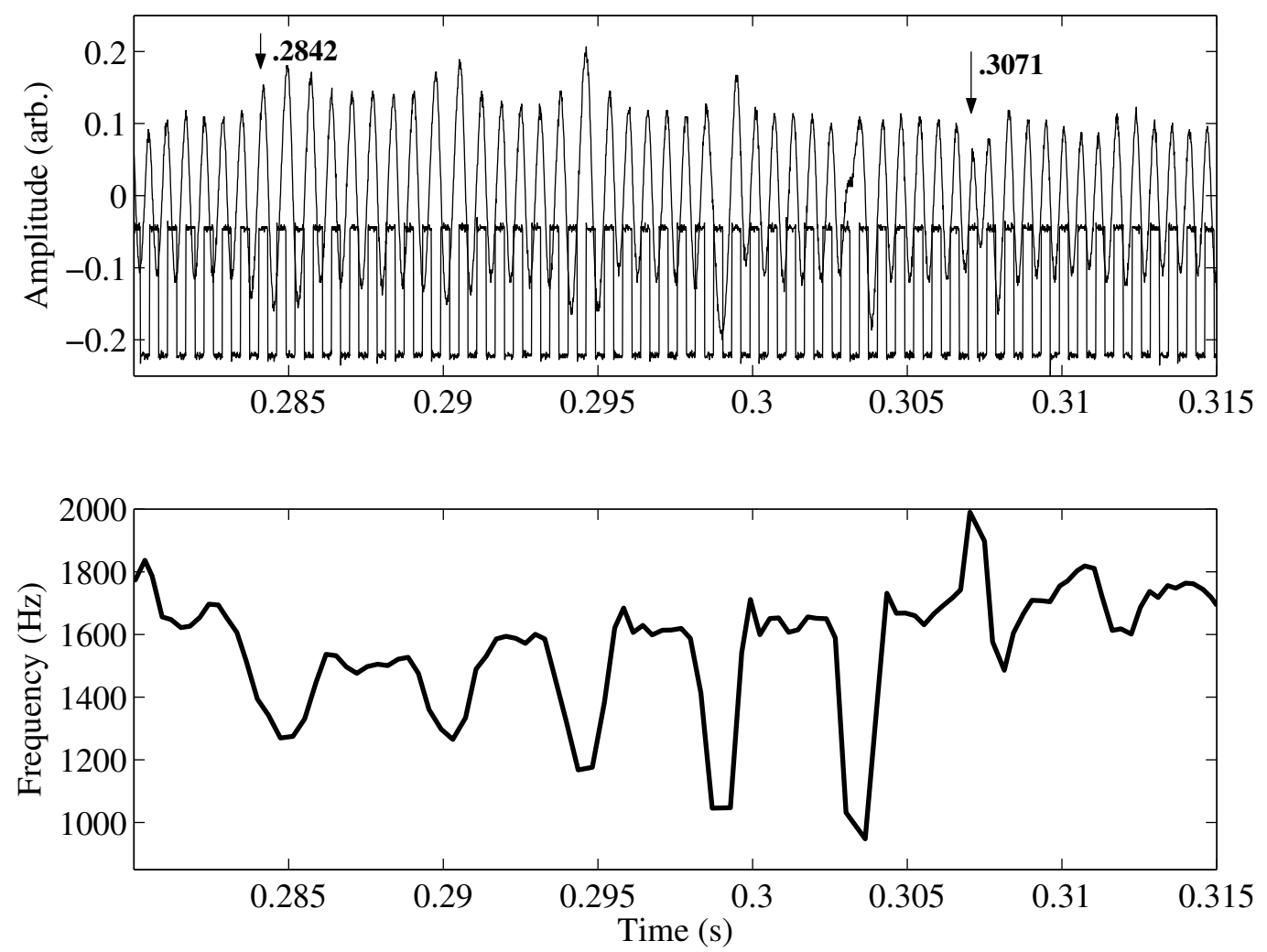

Figure 4.22: Time series close-up of lower mode during periodic dynamics modulation. The beginning of the lower frequency part of the cycle begins when the phase of the luminosity signal changes quickly at $t=0.2842 \mathrm{~s}$, and ends at $t=0.3071 \mathrm{~s}$. The signal almost snaps out of its lower mode at $t=0.3031 \mathrm{~s}$, but appears to remain for one more beat. During the time spent in the lower mode, the amplitude beats with a period of $0.00483 \mathrm{~s}$. The driving frequency, $f_{i}$, was set to $1700 \mathrm{~Hz}$. 

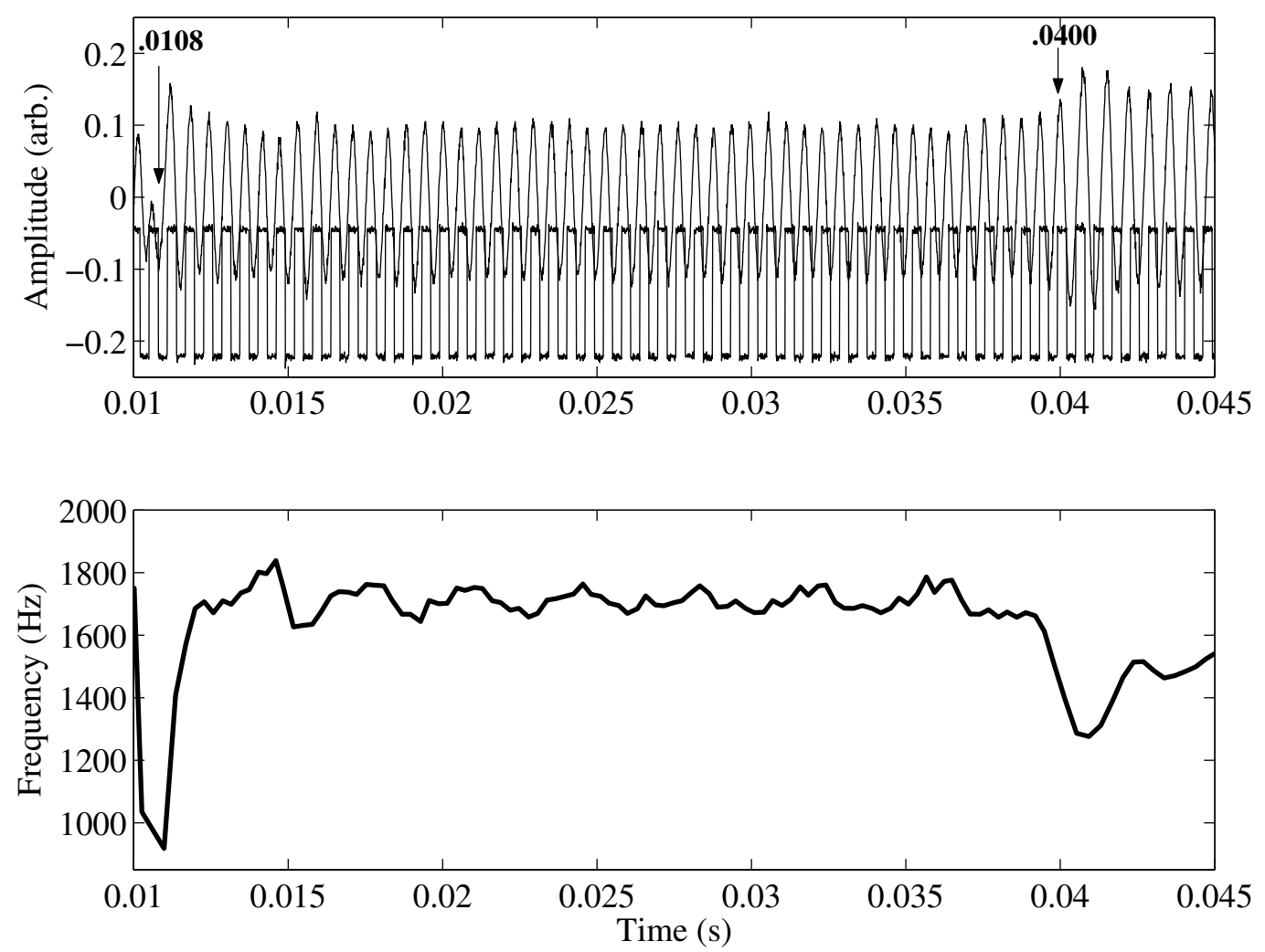

Figure 4.23: Time series close-up of higher mode during periodic dynamics modulation. The beginning of the higher frequency part of the cycle begins when the phase of the luminosity signal changes quickly at $t=0.0108 \mathrm{~s}$, and ends at $t=0.0400 \mathrm{~s}$. During the time spent in the higher mode, the amplitude does not beat in as obvious a manner as the example shown in Figure 4.21 for a lower driving frequency, but the instantaneous frequency shows seven beat periods. The driving frequency, $f_{i}$, was set to $1700 \mathrm{~Hz}$. 

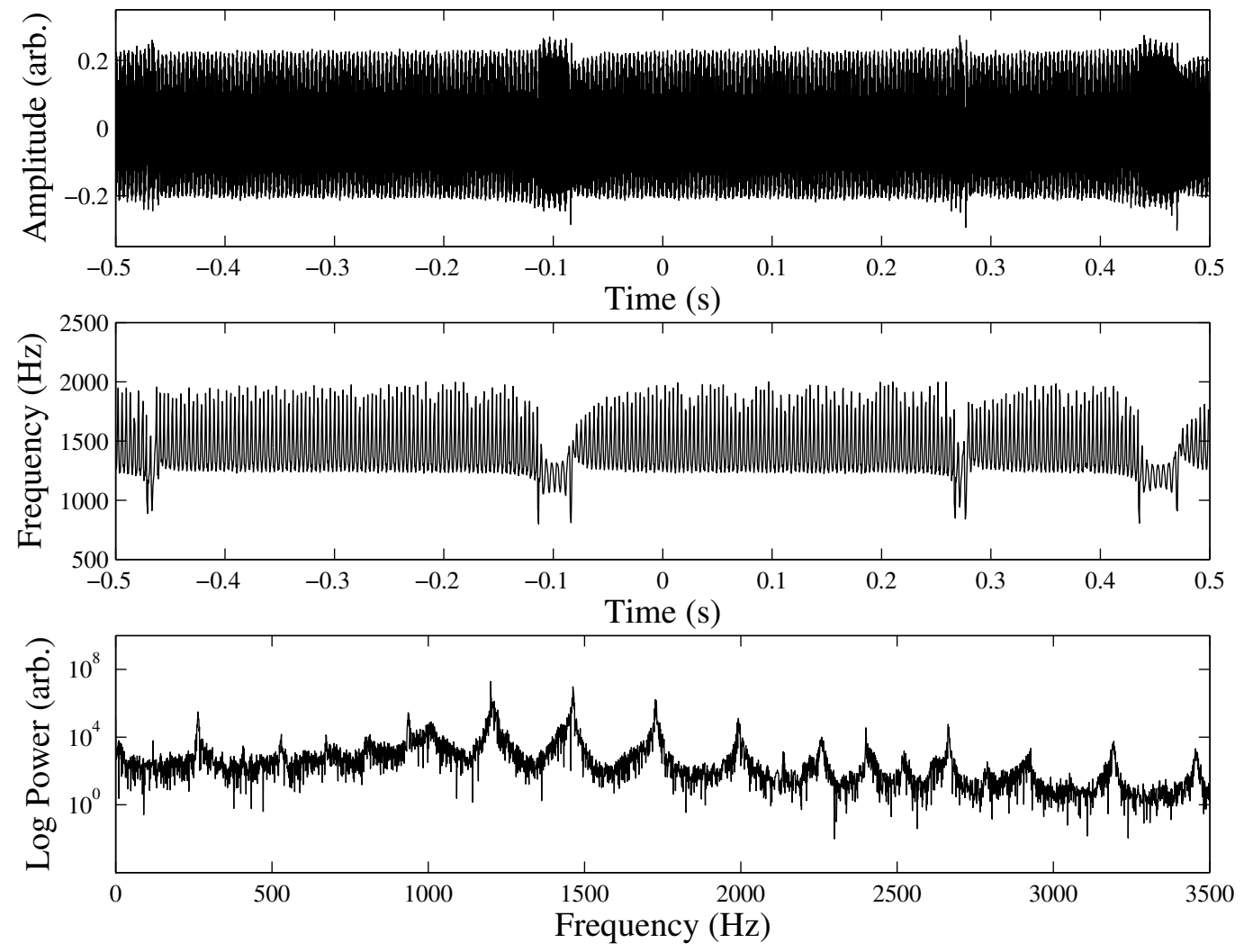

Figure 4.24: A representative example of downward dynamics modulation. The time series (top), instantaneous frequency (center) and power spectrum (bottom) of downward dynamics modulation are shown here. The driving frequency $\left(f_{i}\right)$ was $1200 \mathrm{~Hz}$. The modulation is taking place between the $f 7$ and $f 6$ modes. The discharge current was set to $9.10 \mathrm{~mA}$. 
a

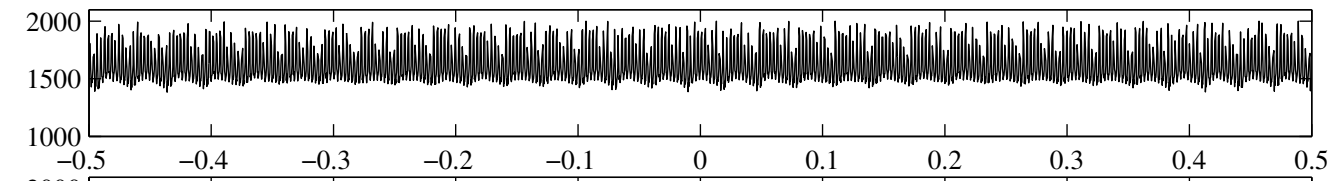

b
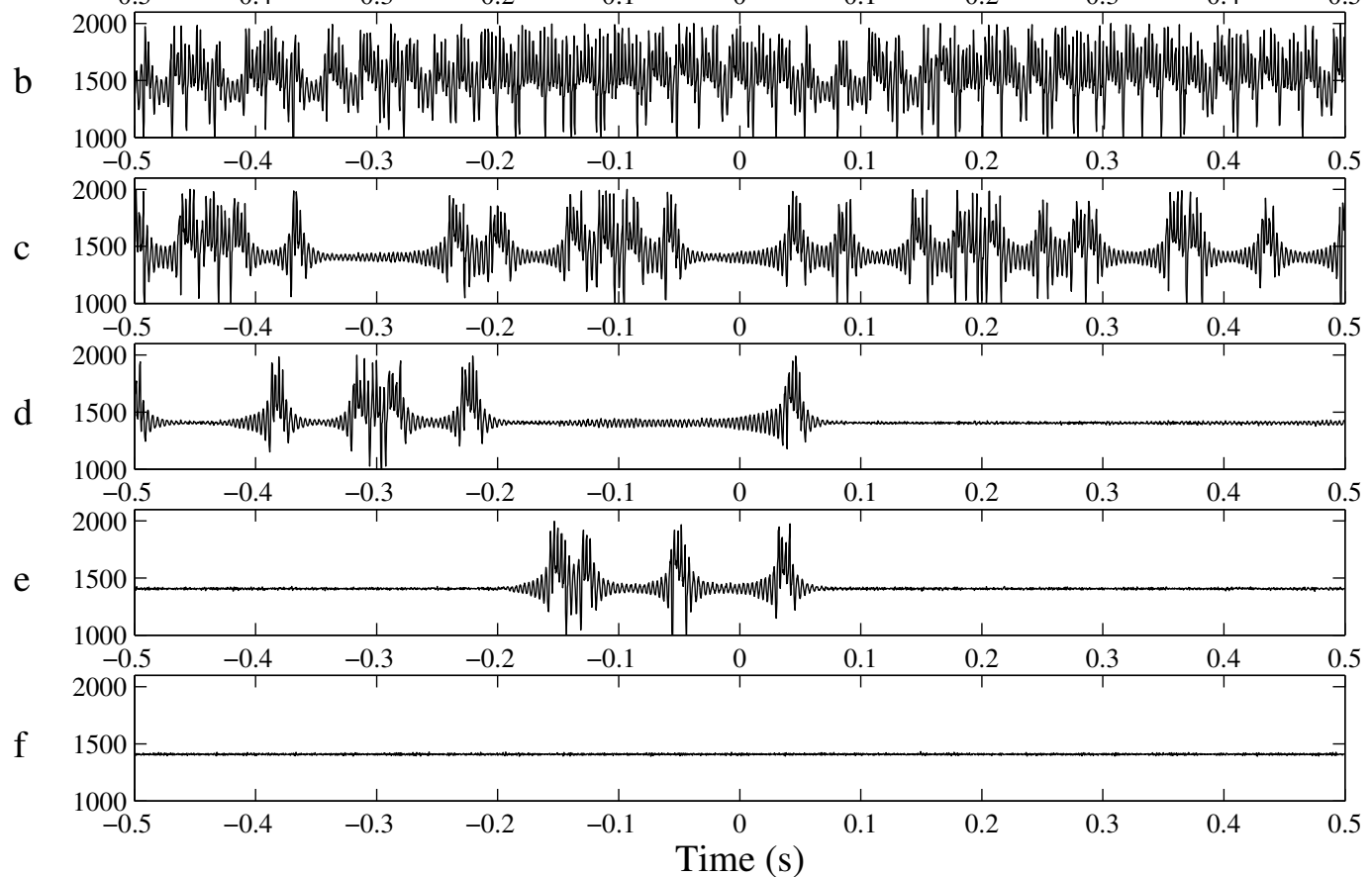

Figure 4.25: A sequence of instantaneous frequency series for downward dynamics modulation as a function of driving frequency. The $y$-axis of each plot shows the instantaneous frequency in Hz. Beginning at the top, the driving frequency $\left(f_{i}\right)$ is 1385 (a), 1395 (b), 1403 (c), 1405 (d), 1406 (e) and 1409 (f) Hz. The modulation is taking place between the $f 7$ and $f 6$ modes. Panels (a) through (e) represent aperiodic downward dynamics modulation, and panel (f) represents entrainment of the $f 6$ mode. The discharge current was set to $9.10 \mathrm{~mA}$. Entrainment occurred at $1409 \mathrm{~Hz}$. 
a

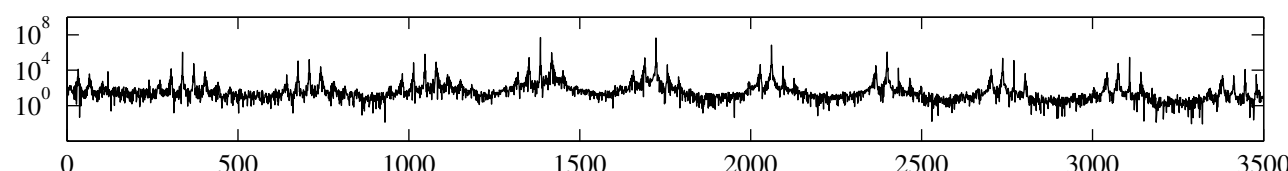

b

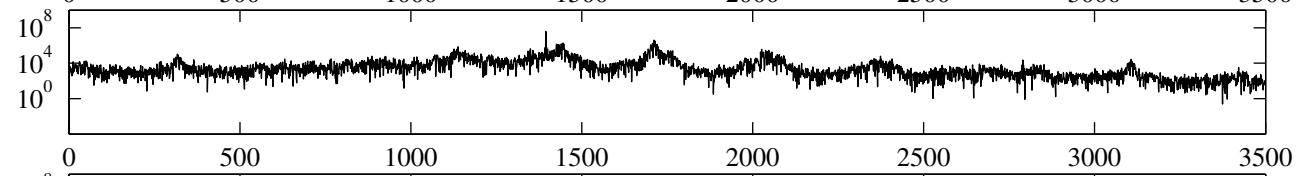

c

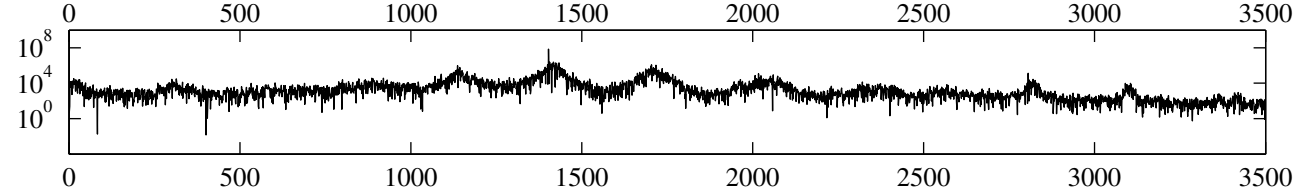

d

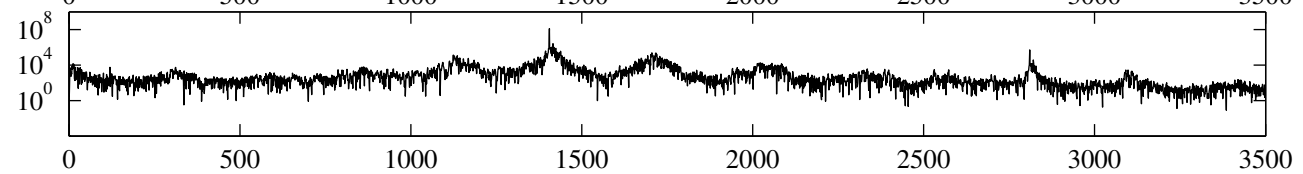

$\mathrm{e}$

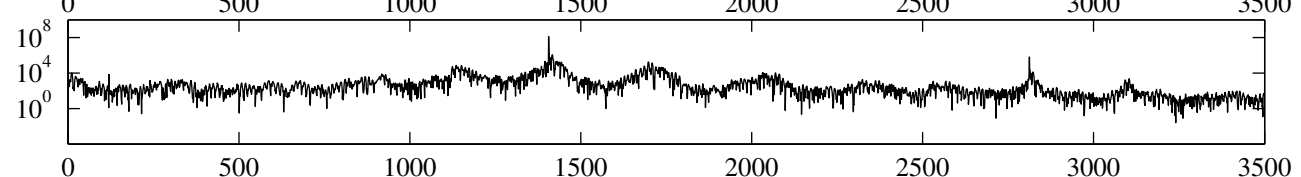

f

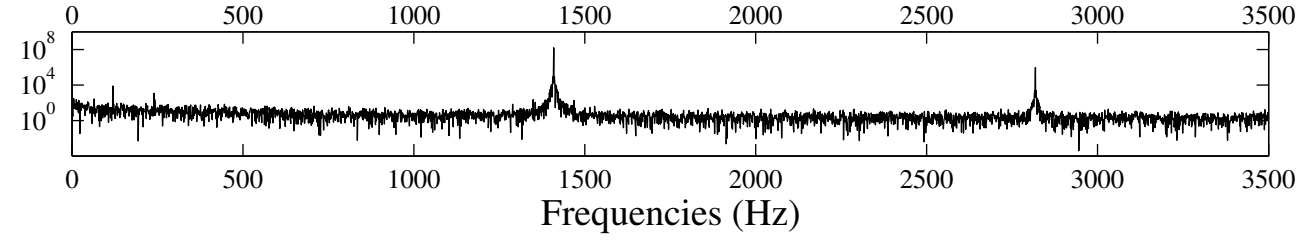

Figure 4.26: A sequence of power spectra for downward dynamics modulation as a function of driving frequency. The $y$-axis of each plot shows the instantaneous frequency in Hz. Beginning at the top, the driving frequency $\left(f_{i}\right)$ is 1385 (a), 1395 (b), 1403 (c), 1405 (d), 1406 (e) and 1409 (f) Hz. The modulation is taking place between the $f 7$ and $f 6$ modes. Panels (a) through (e) represent aperiodic downward dynamics modulation, and panel (f) represents entrainment of the $f 6$ mode. The discharge current was set to $9.10 \mathrm{~mA}$. Entrainment occurred at $1409 \mathrm{~Hz}$. 
a

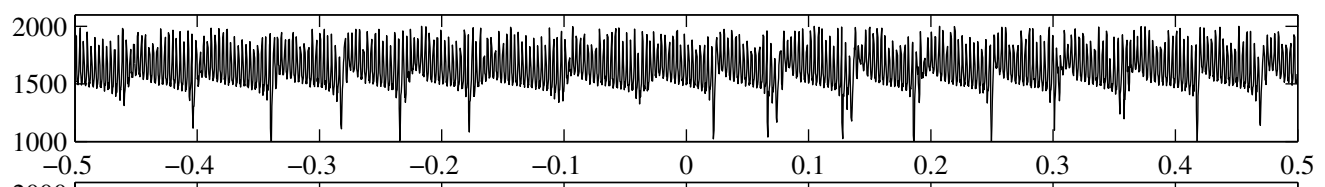

b
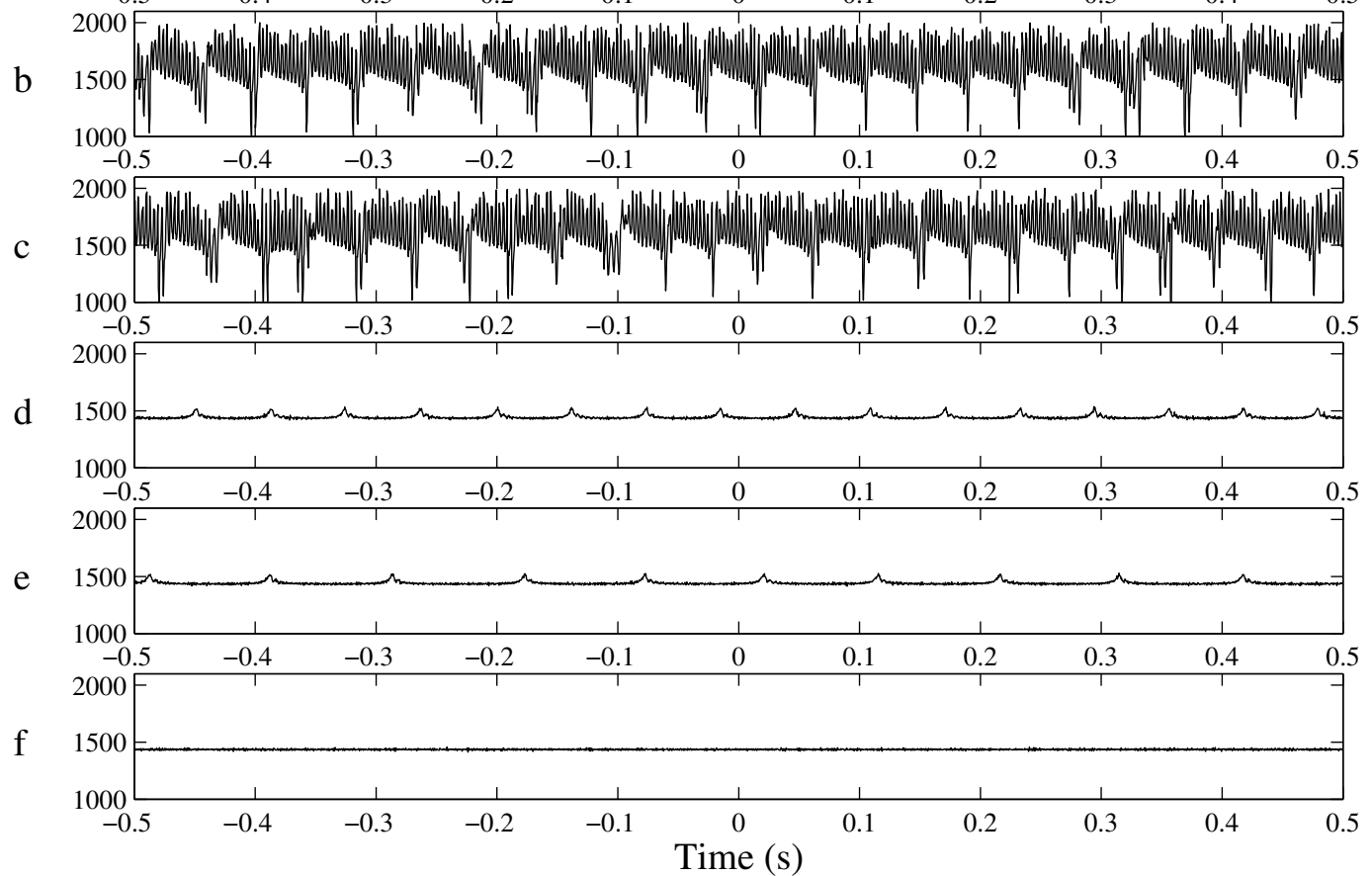

Figure 4.27: A sequence of instantaneous frequency series for downward dynamics modulation as a function of driving frequency. The $y$-axis of each plot shows the instantaneous frequency in Hz. Beginning at the top, the driving frequency $\left(f_{i}\right)$ is 1420 (a), 1423 (b), 1426 (c), 1429 (d), 1432 (e) and 1435 (f) Hz. The modulation is taking place between the $f 7$ and $f 6$ modes. Panels (a) through (c) represent downward dynamics modulation. Panels (d) and (e) represent temporal periodic pulling of the $f 6$ mode only and panel (f) represents entrainment. The discharge current was set to $9.10 \mathrm{~mA}$. Entrainment occurred at $1435 \mathrm{~Hz}$. 
a

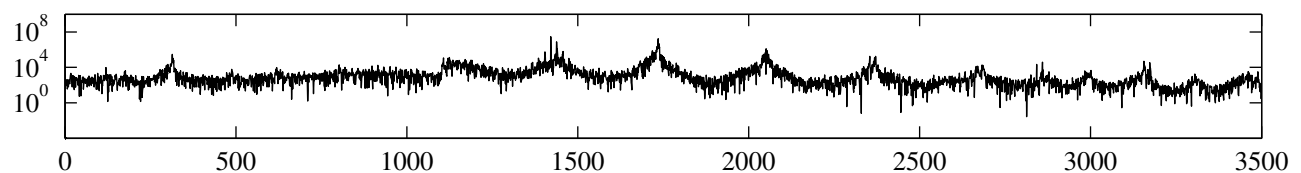

b

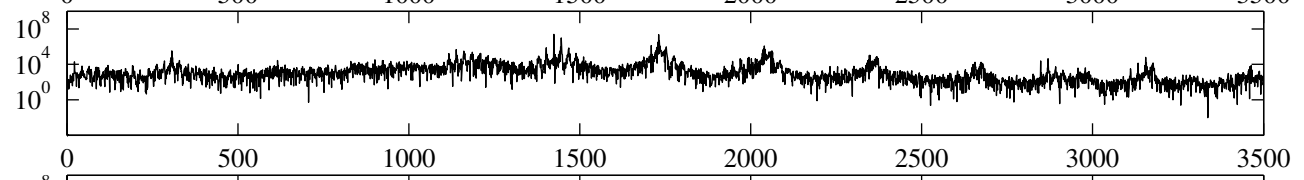

c

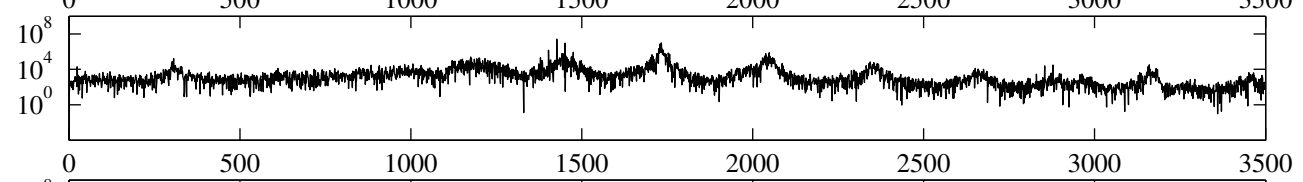

d

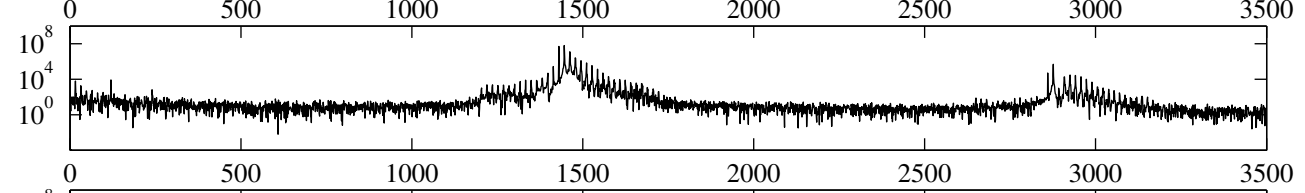

e

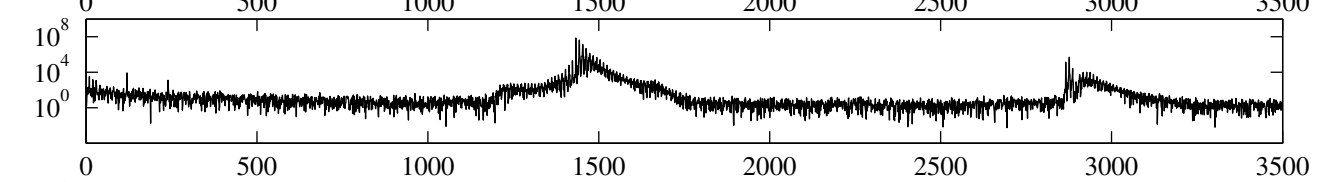

$\mathrm{f}$

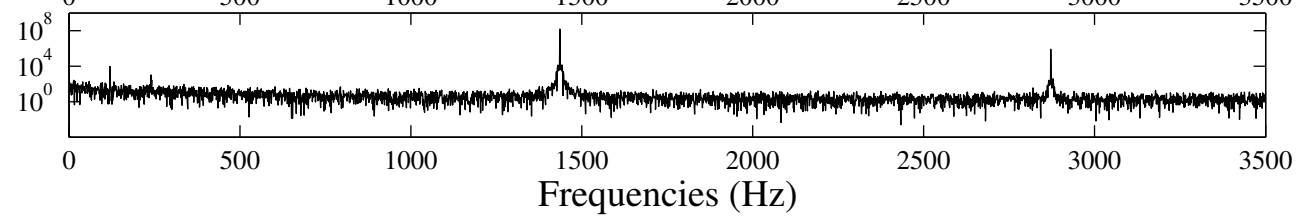

Figure 4.28: A sequence of power spectra for downward dynamics modulation as a function of driving frequency. The $y$-axis of each plot shows the instantaneous frequency in Hz. Beginning at the top, the driving frequency $\left(f_{i}\right)$ is 1420 (a), 1423 (b), 1426 (c), 1429 (d), 1432 (e) and 1435 (f) Hz. The modulation is taking place between the $f 7$ and $f 6$ modes. Panels (a) through (c) represent downward dynamics modulation. Panels (d) and (e) represent temporal periodic pulling of the $f 6$ mode only and panel (f) represents entrainment. The discharge current was set to 9.10 mA. Entrainment occurred at $1435 \mathrm{~Hz}$. 


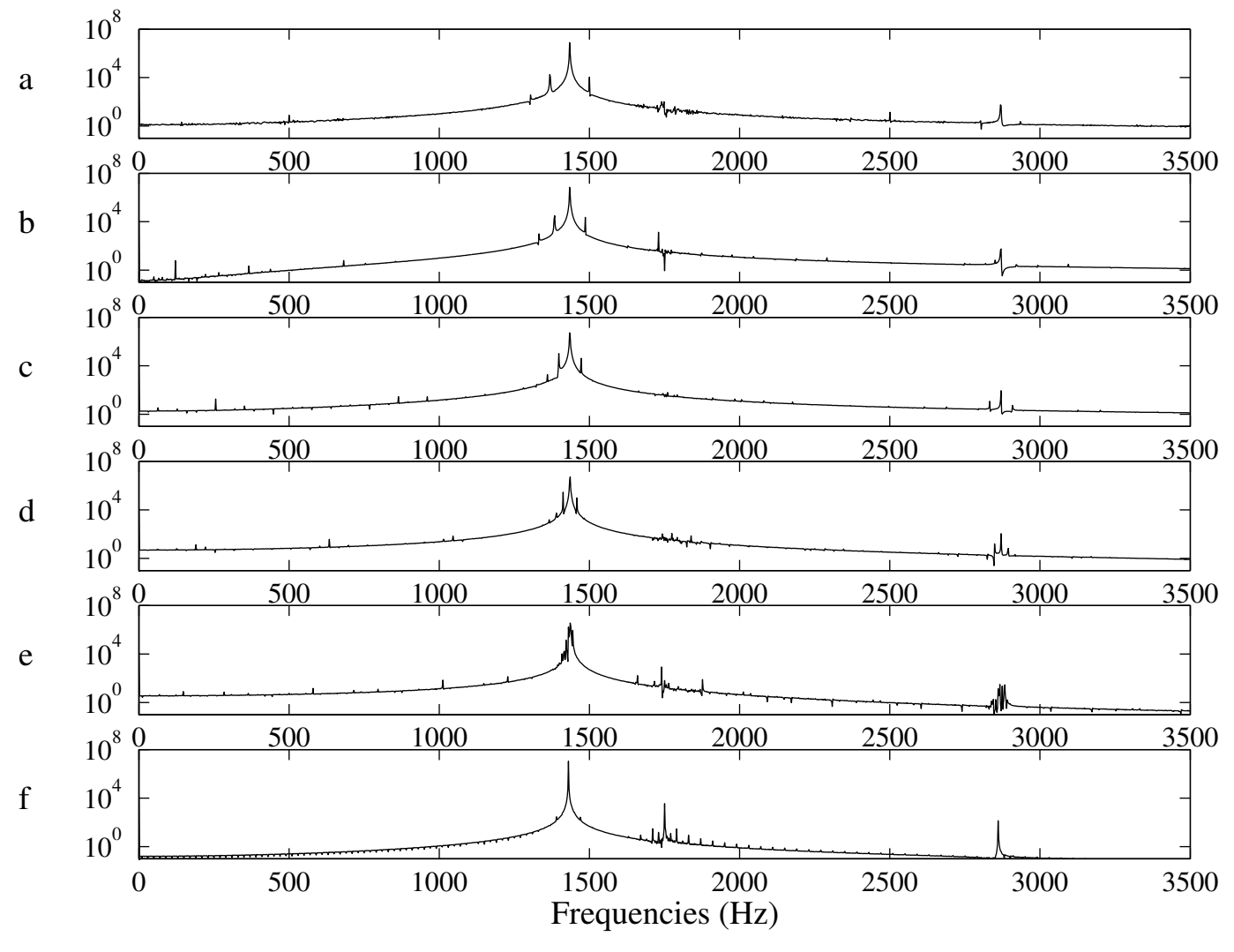

Figure 5.1: A good example of temporal periodic pulling in the model. Here, $\epsilon_{1}=0.1, \beta_{1}=1$, and $M=0.03$. The coupling parameters were set at $\gamma_{1}=0.025$ and $\gamma_{2}=0.22$. $\omega_{1}=2 \pi \times 1450$ and $\omega_{2}=2 \pi \times 1740$, the values from the experimental data. For this one case, $\epsilon_{2}=\beta_{2}=M_{2}=0$, to keep the second mode separate. $f_{i}$ was 1500 (a), 1486 (b), 1472 (c), 1458 (d) 1444 (e), and 1430 (f) Hz. Panel (f) represents entrainment rather than periodic pulling. The qualitative behavior of the model (Figure 5.1) is very similar to that of the experiment seen in Figure 3.4. 


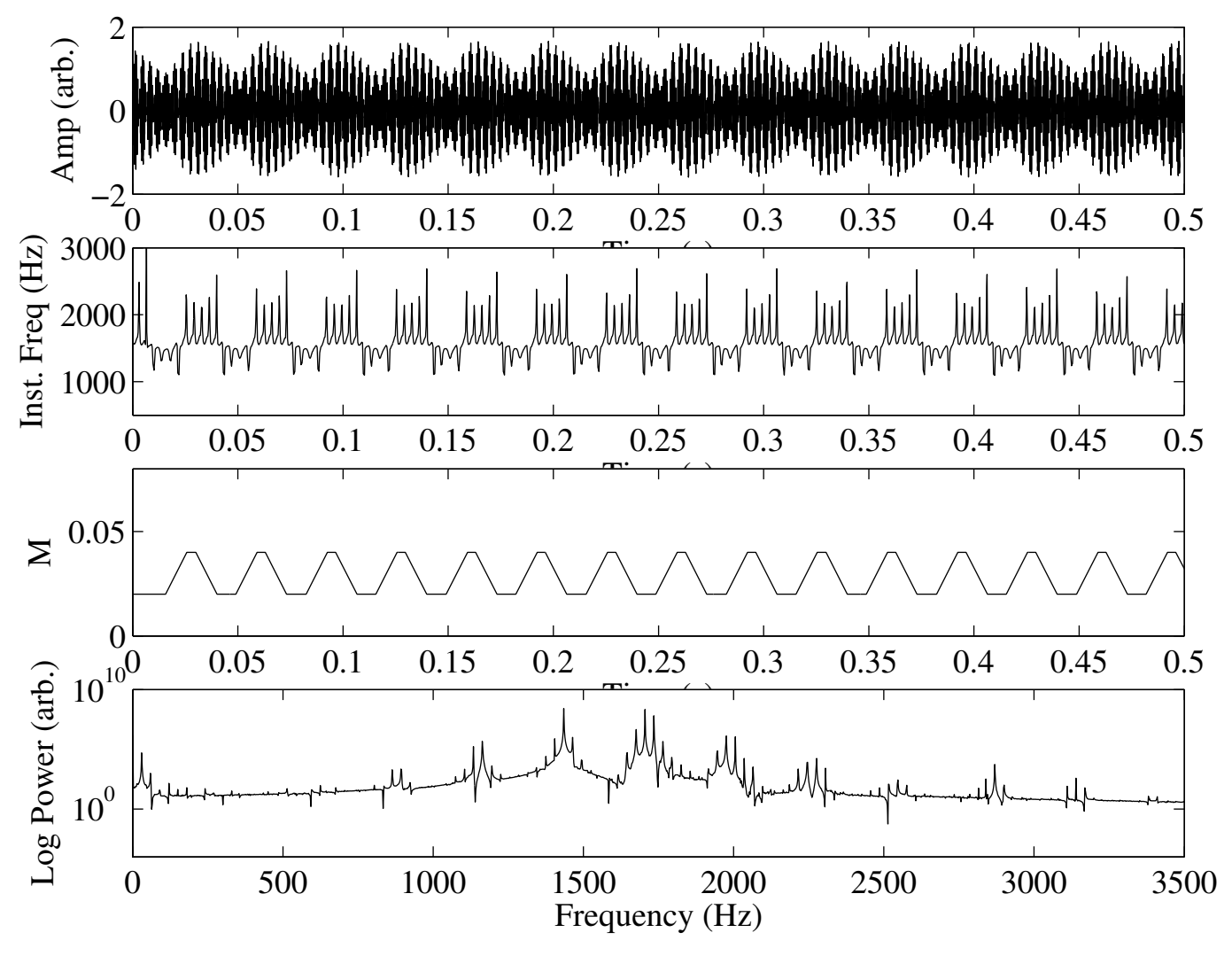

Figure 5.2: Simulated dynamics modulation. Here, $\epsilon=0.1$ and $\beta=1$. The coupling parameters were set at $\gamma_{1}=0.025$ and $\gamma_{2}=0.22$ and the mode frequencies were set at $\omega_{1}=2 \pi \times 1450$ and $\omega_{2}=2 \pi \times 1740$. The driving frequency, $f_{i}$, was $1705 \mathrm{~Hz}$. The panels, in order from top to bottom, show the time series, the instantaneous frequency, the value of $M$ used in the simulation in response to the detected mode, and the power spectrum. 

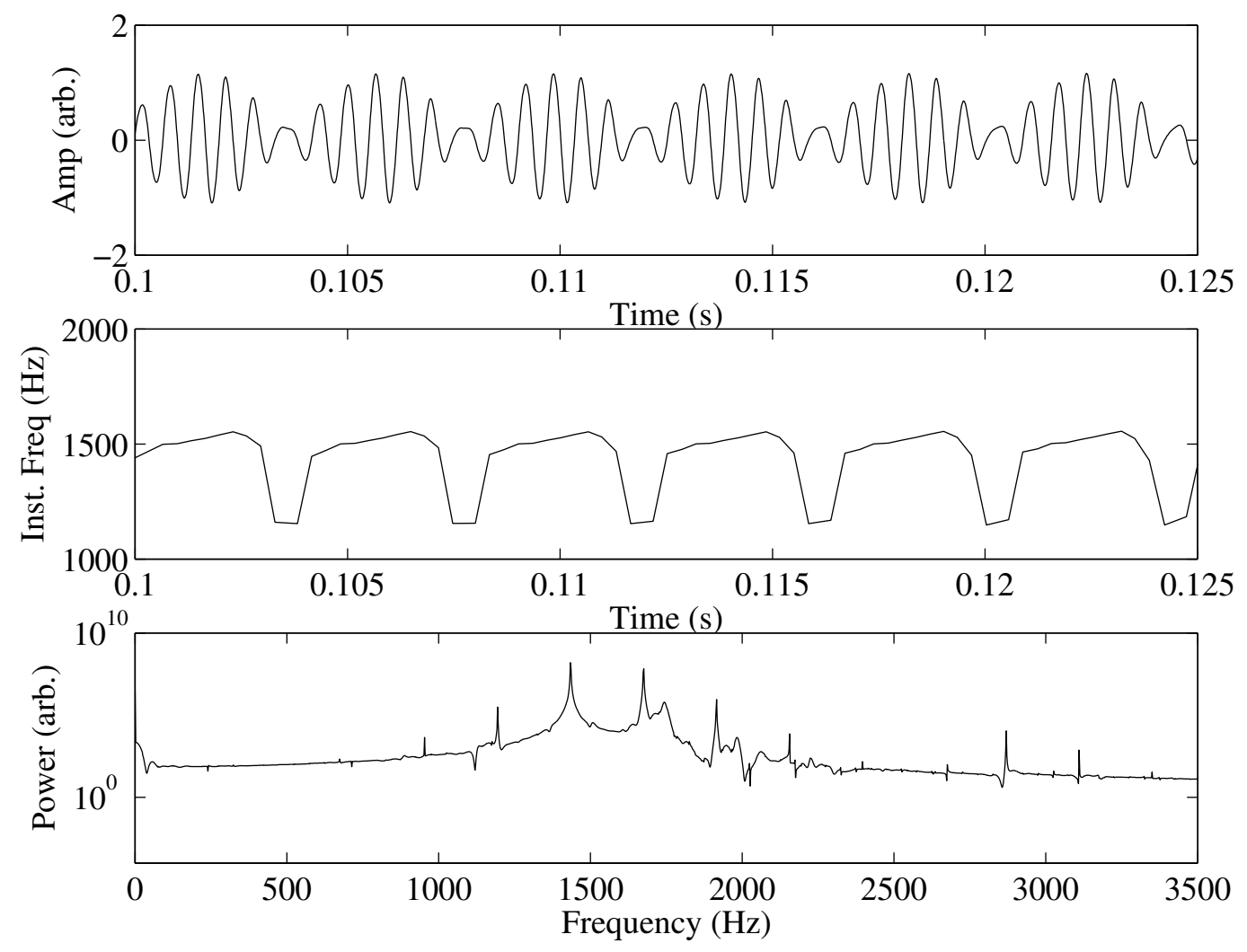

Figure 5.3: Simulated spatiotemporal periodic pulling. $f_{i}$ was set to $1675 \mathrm{~Hz}$. Other inputs were the same as Figure 5.2. The result is an excellent example of spatiotemporal periodic pulling from above. This is shown by the concave-downward instantaneous frequency plot (bottom) which corresponds to the time series presented above it. 


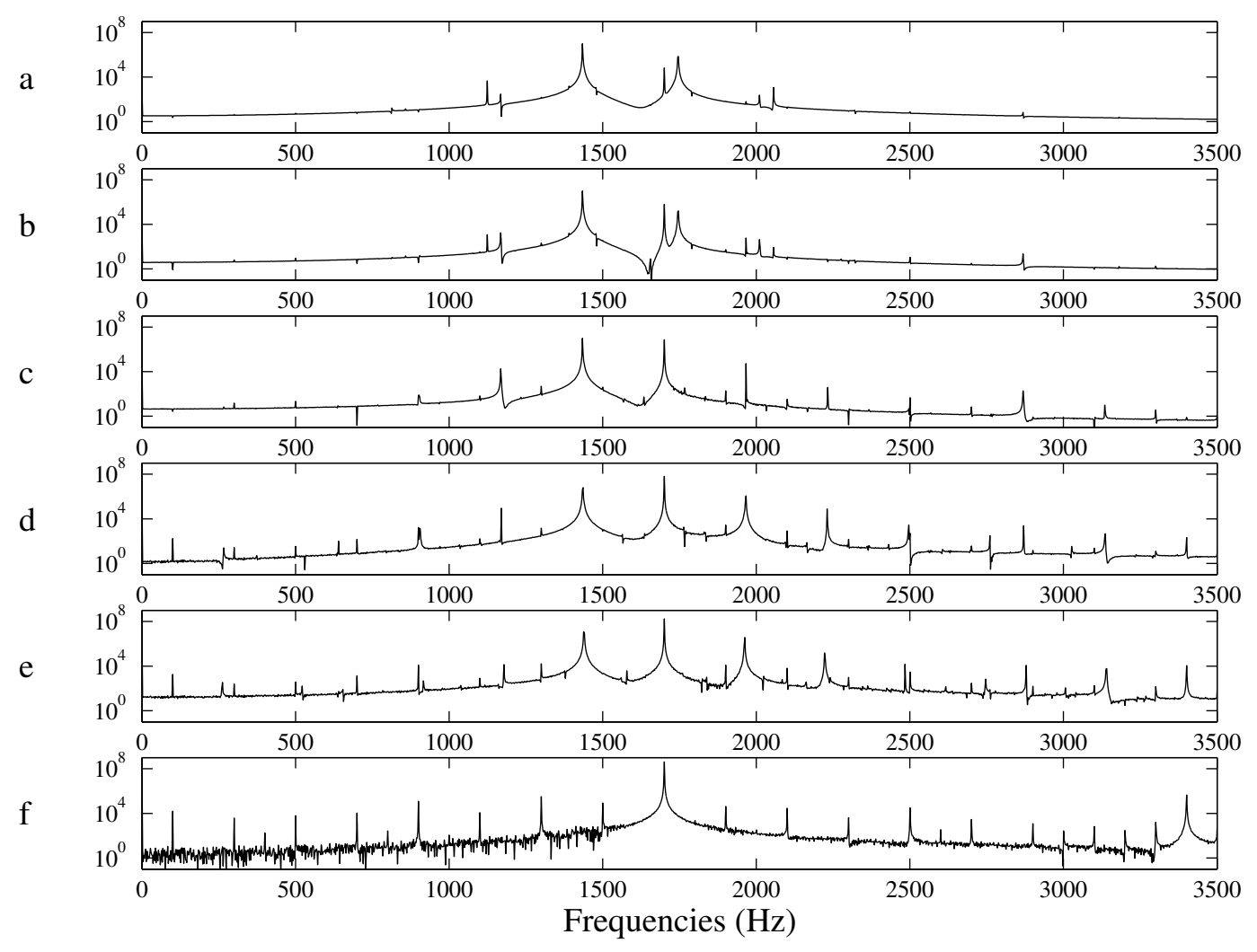

Figure 5.4: Power spectra as a function of $M$. $M$ was set to 0.0028 (a), 0.0091 (b), 0.0295 (c), 0.0955 (d), 0.3090 (e), and 1.000 (f). The remaining parameters were $\epsilon=0.1, \beta=1$, and coupling parameters $\gamma_{1}=0.025$ and $\gamma_{2}=0.22$. The mode frequencies were set at $\omega_{1}=2 \pi \times 1450 \mathrm{~Hz}$ and $\omega_{2}=2 \pi \times 1740 \mathrm{~Hz}$. The driving frequency was set to $f_{i}=1700 \mathrm{~Hz}$. 


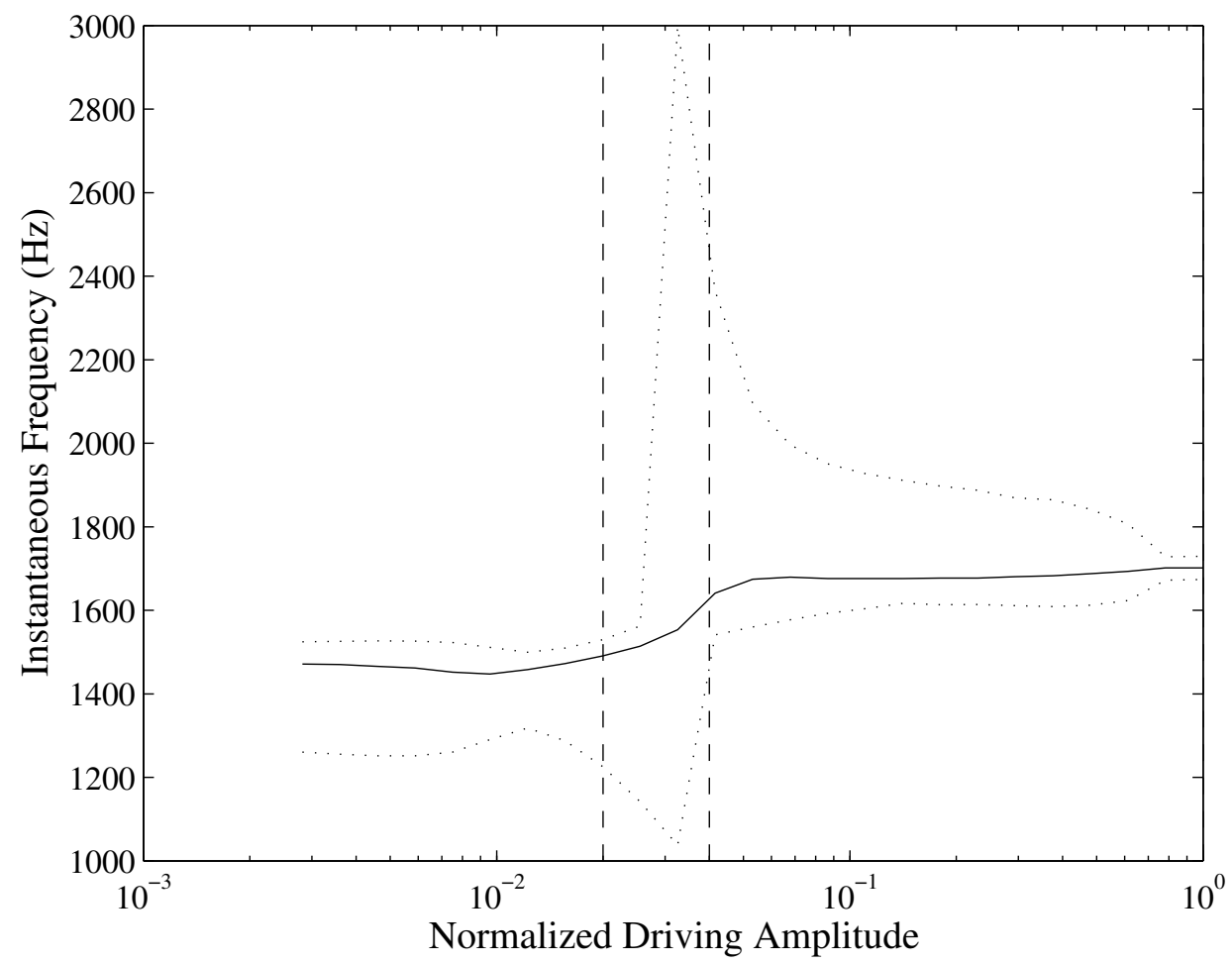

Figure 5.5: Instantaneous frequency trend as a function of $M$. The solid line traces the median instantaneous frequency value as a function of normalized driving amplitude $M$. Dotted lines above and below represent extreme values. The mode frequencies were set at $\omega_{1}=2 \pi \times 1450 \mathrm{~Hz}$ and $\omega_{2}=2 \pi \times 1740 \mathrm{~Hz}$. The driving frequency was set to $f_{i}=1700 \mathrm{~Hz}$. In the limit of low driving amplitudes, the median instantaneous frequency stays near $1450 \mathrm{~Hz}$. In the limit of high driving amplitudes, the median instantaneous frequency equals the driving frequency. The dashed lines represent $M_{H}$ (left) and $M_{L}=2 M_{H}$ (right) used in the model. Parameters were $\epsilon=0.1, \beta=1$, and coupling parameters $\gamma_{1}=0.025$ and $\gamma_{2}=0.22$. 


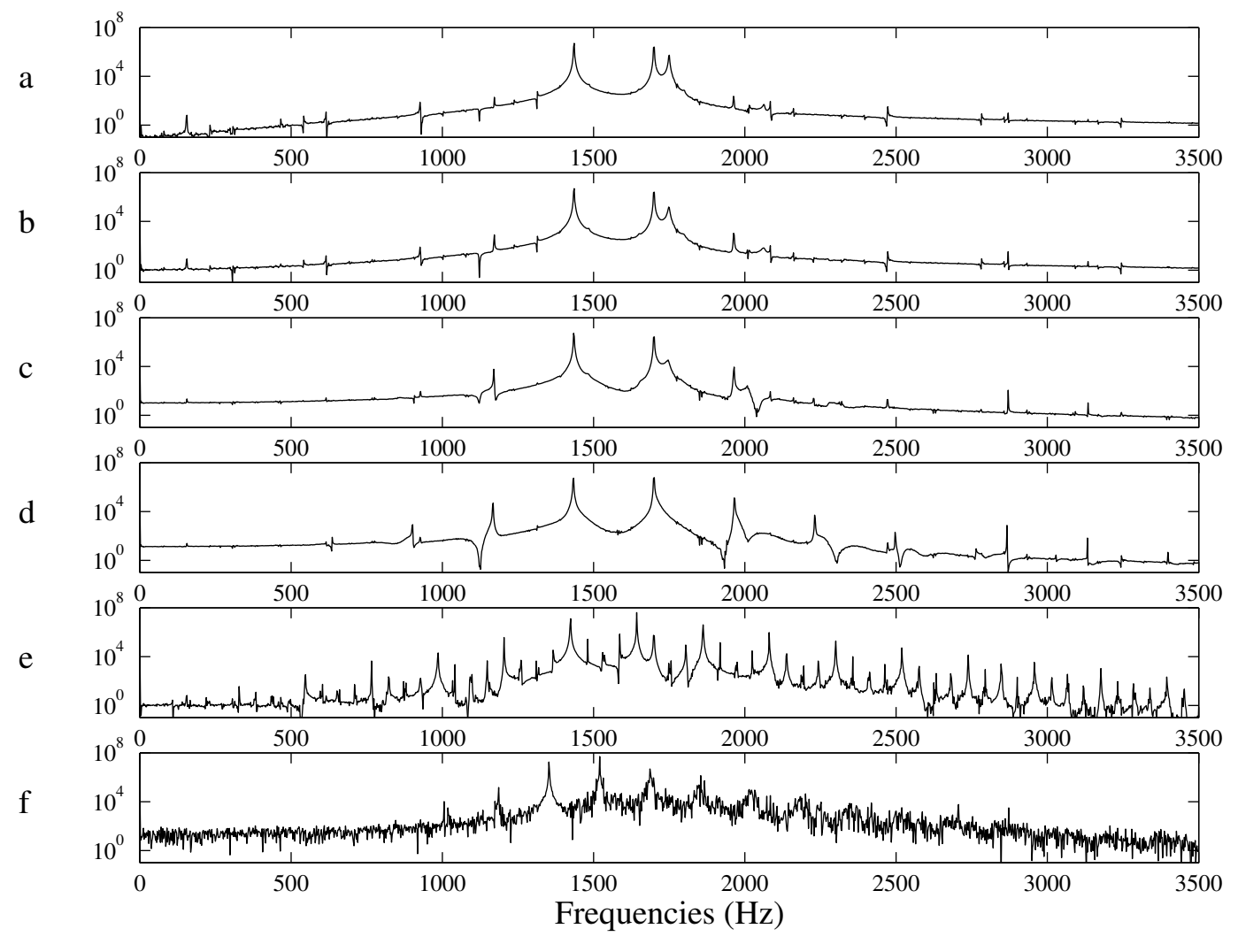

Figure 5.6: Power spectra as a function of $\epsilon . \epsilon$ was set to 0.0100 (a), 0.0251 (b), 0.0631 (c), 0.1585 (d), 0.3981 (e) and 1.000 (f). Parameters were $M=0.03, \beta=1$, and coupling parameters $\gamma_{1}=0.025$ and $\gamma_{2}=0.22$. The mode frequencies were set at $\omega_{1}=2 \pi \times 1450 \mathrm{~Hz}$ and $\omega_{2}=2 \pi \times 1740 \mathrm{~Hz}$. The driving frequency was set to $f_{i}=1700 \mathrm{~Hz}$. 


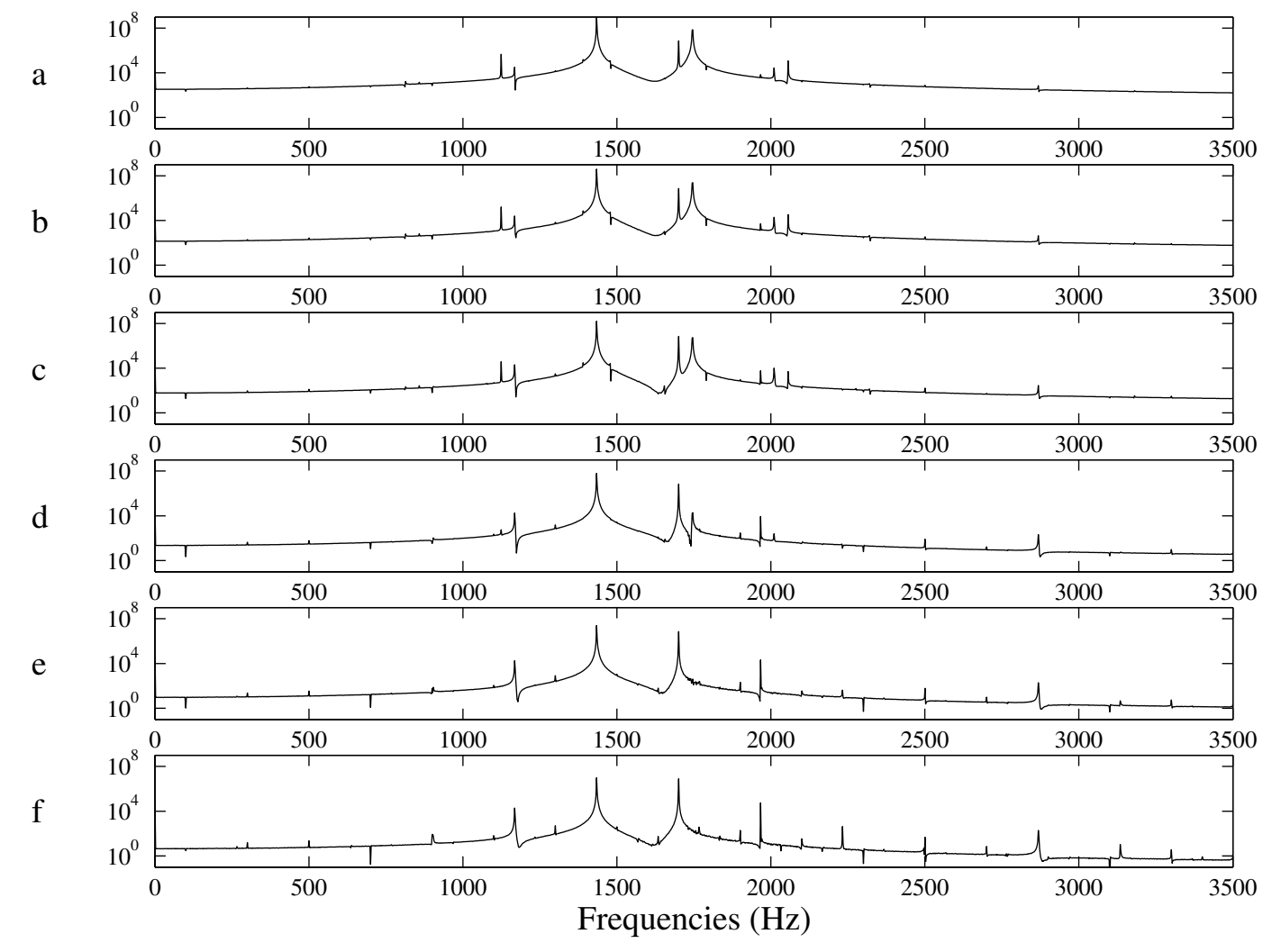

Figure 5.7: Power spectra as a function of $\beta$. $\beta$ was set to 0.0100 (a), 0.0251 (b), 0.0631 (c), 0.1585 (d), 0.3981 (e) and 1.000 (f). Parameters were $M=0.03, \epsilon=0.1$, and coupling parameters $\gamma_{1}=$ 0.025 and $\gamma_{2}=0.22$. The mode frequencies were set at $\omega_{1}=2 \pi \times 1450 \mathrm{~Hz}$ and $\omega_{2}=2 \pi \times 1740 \mathrm{~Hz}$. The driving frequency was set to $f_{i}=1700 \mathrm{~Hz}$. 


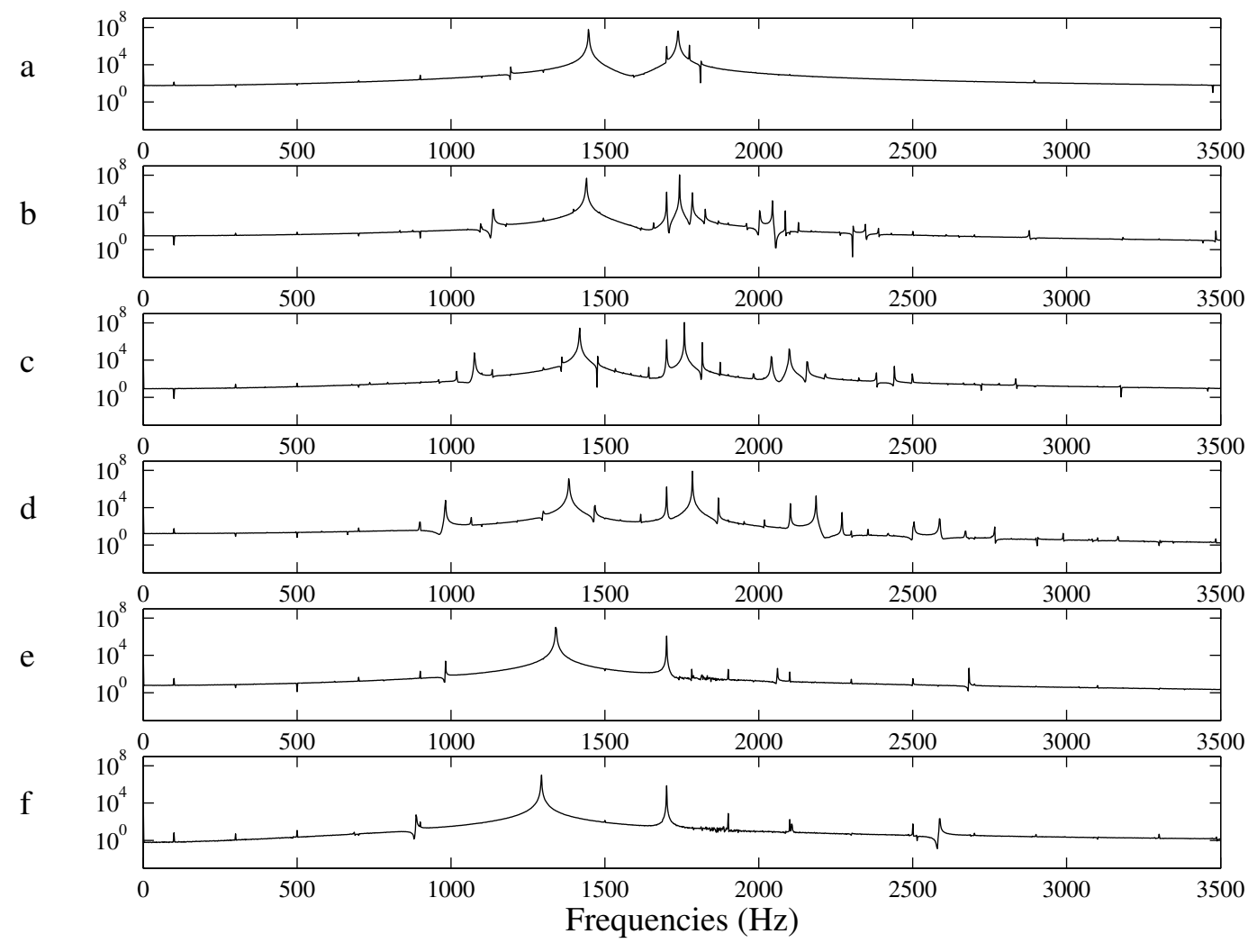

Figure 5.8: Dependence on coupling $\gamma$, with $\gamma_{1}=\gamma_{2}=\gamma$. The values used for $\gamma$ in this case are 0.001 (a), 0.0608 (b), 0.1206 (c), 0.1804 (d), 0.2402 (e), and 0.3000 (f). Parameters were $M=0.03$, $\epsilon=0.1$, and $\beta=1$. The mode frequencies were set at $\omega_{1}=2 \pi \times 1450 \mathrm{~Hz}$ and $\omega_{2}=2 \pi \times 1740 \mathrm{~Hz}$. The driving frequency was set to $f_{i}=1700 \mathrm{~Hz}$. 


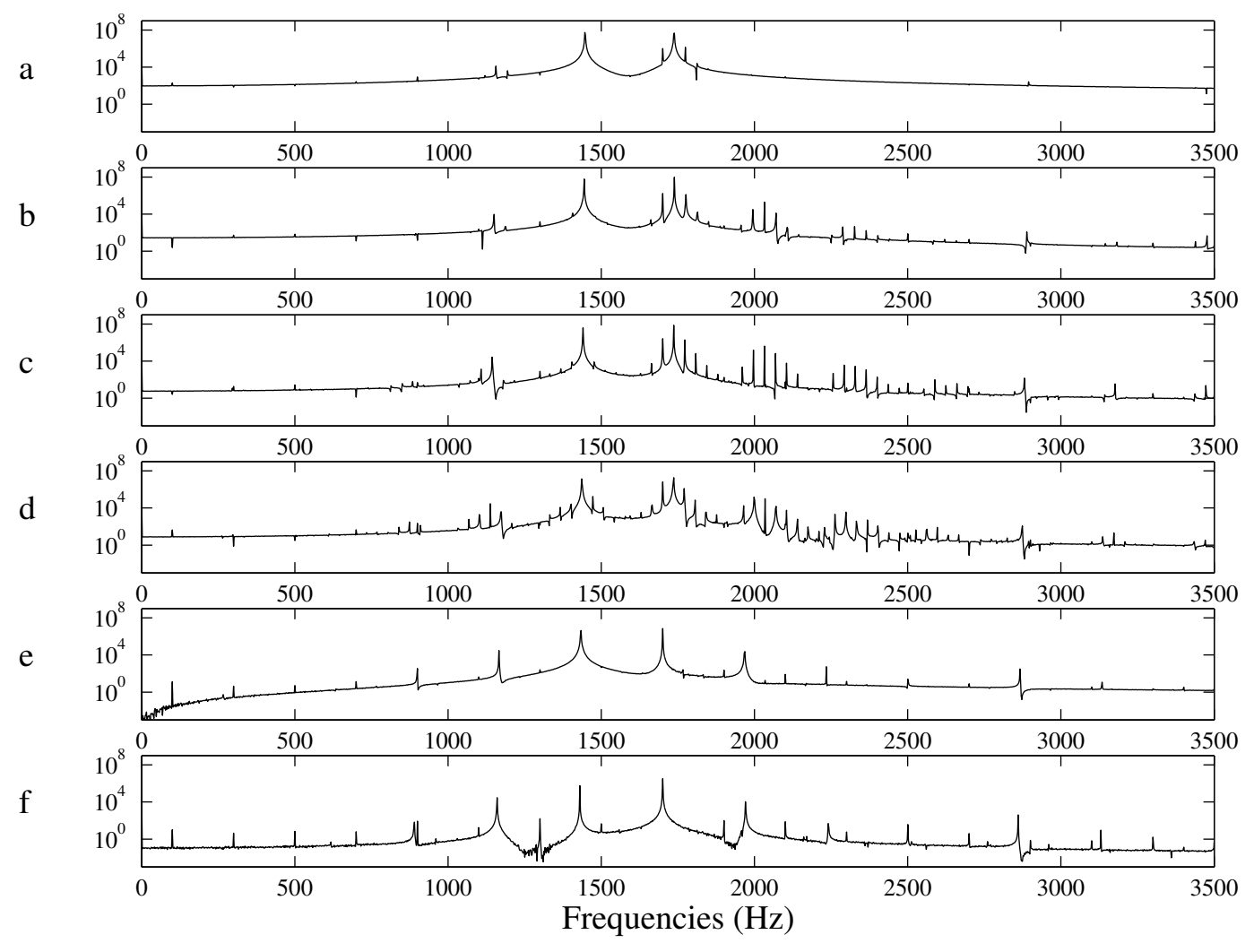

Figure 5.9: Dependence on coupling $\gamma_{2}$, with $\gamma_{1}=0.025$. $\gamma_{2}$ was set to 0.0003 (a), 0.0602 (b), 0.1202 (c), 0.1801 (d), 0.2401 (e), and 0.3000 (f). This corresponds to $\gamma_{2} / \gamma_{1}$ ranging from 0.01 to 12.0. Parameters were $M=0.03, \epsilon=0.1$, and $\beta=1$. The mode frequencies were set at $\omega_{1}=2 \pi \times 1450 \mathrm{~Hz}$ and $\omega_{2}=2 \pi \times 1740 \mathrm{~Hz}$. The driving frequency was set to $f_{i}=1700 \mathrm{~Hz}$ 


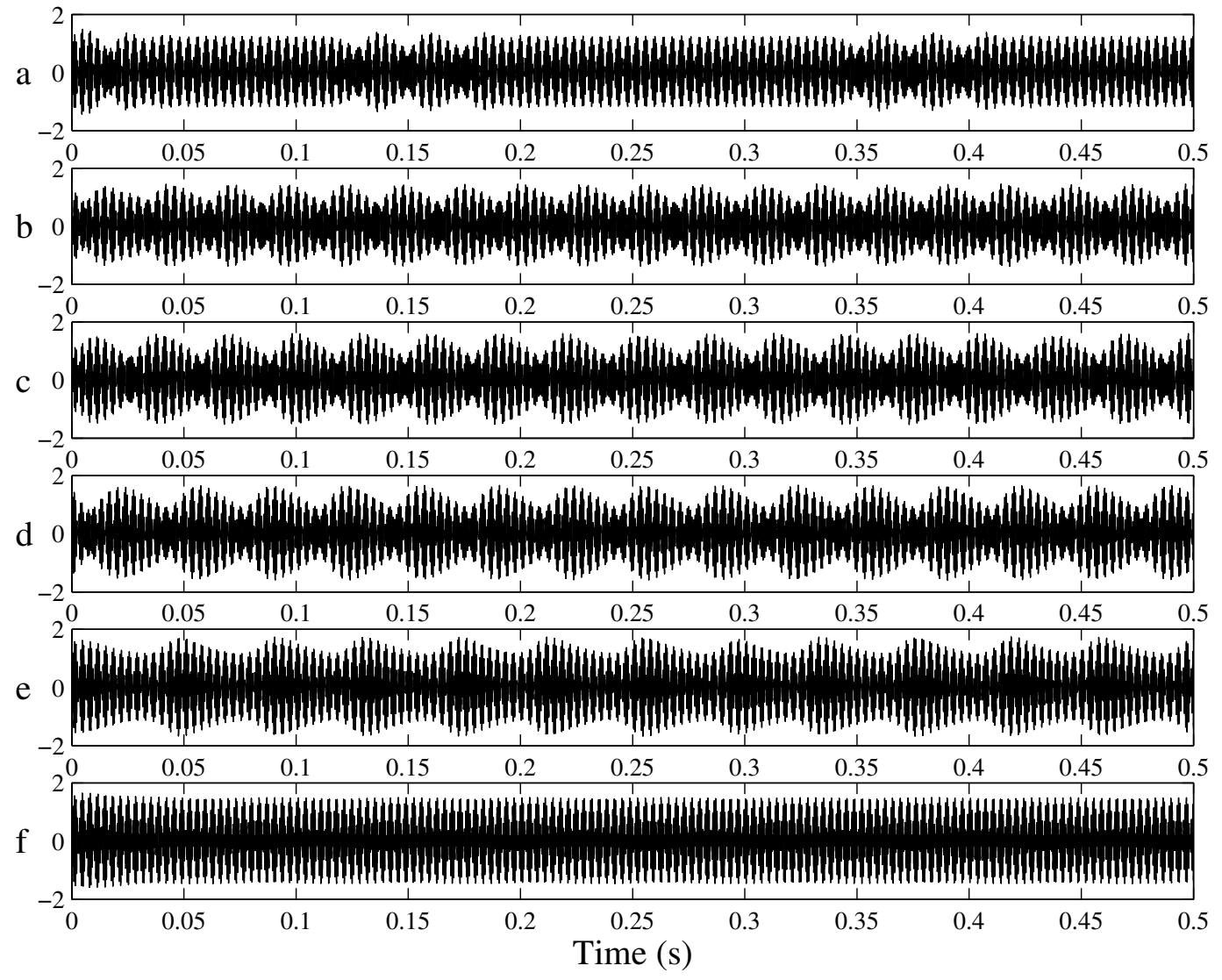

Figure 5.10: Simulated dynamics modulation as a function of $f_{i}$, time series. The driving frequency, $f_{i}$, was 1685 (a), 1690 (b), 1700 (c), 1705 (d), 1715 (e), and 1725 (f). Other parameters were $M$ toggling between 0.02 (when high mode detected) and 0.04 (when low mode detected), $\epsilon=0.1$, and $\beta=1$. The coupling parameters were $\gamma_{1}=0.025$ and $\gamma_{2}=0.22$. The mode frequencies were set at $\omega_{1}=2 \pi \times 1450 \mathrm{~Hz}$ and $\omega_{2}=2 \pi \times 1740 \mathrm{~Hz}$. 


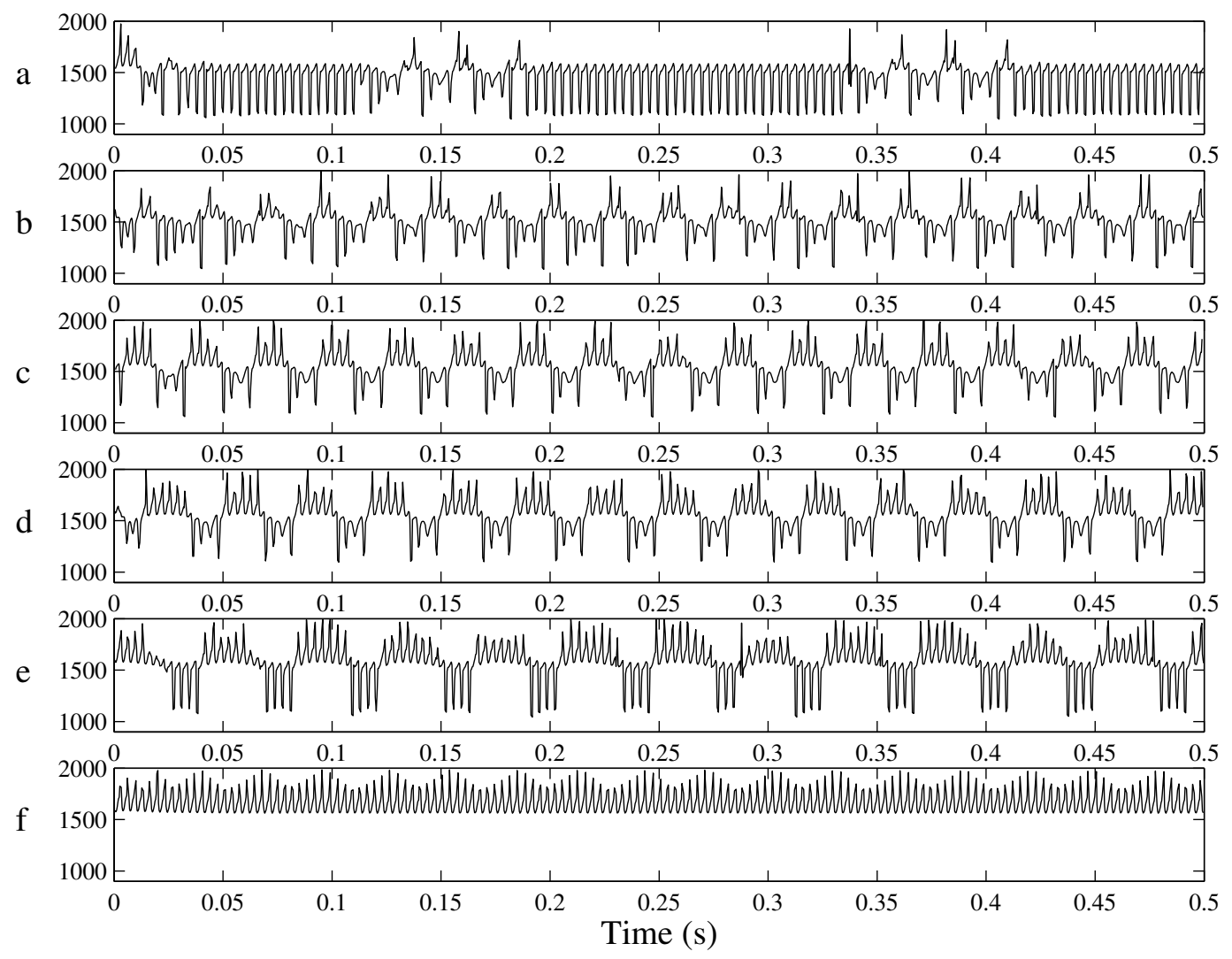

Figure 5.11: Simulated dynamics modulation as a function of $f_{i}$, instantaneous frequency. The driving frequency, $f_{i}$, was 1685 (a), 1690 (b), 1700 (c), 1705 (d), 1715 (e), and 1725 (f). Other parameters were $M$ toggling between 0.02 (when high mode detected) and 0.04 (when low mode detected), $\epsilon=0.1$, and $\beta=1$. The coupling parameters were $\gamma_{1}=0.025$ and $\gamma_{2}=0.22$. The mode frequencies were set at $\omega_{1}=2 \pi \times 1450 \mathrm{~Hz}$ and $\omega_{2}=2 \pi \times 1740 \mathrm{~Hz}$. 


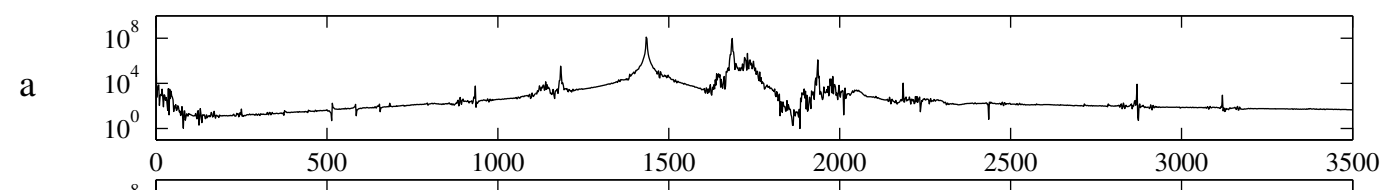

b

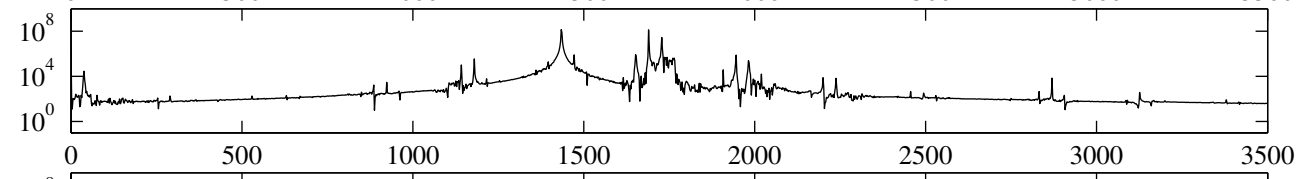

$\mathrm{c}$

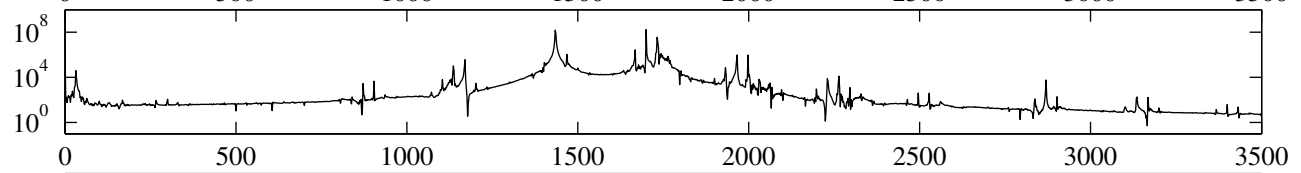

d

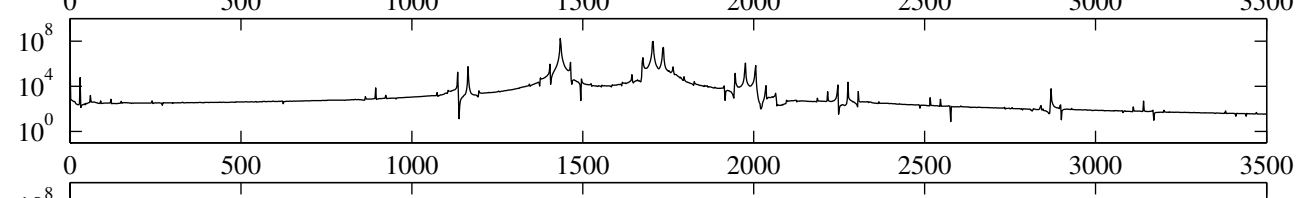

e

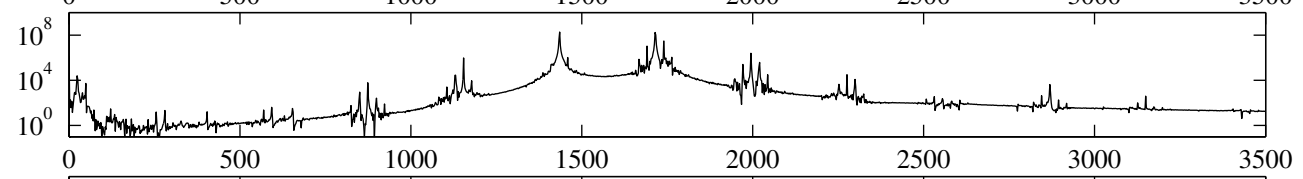

f

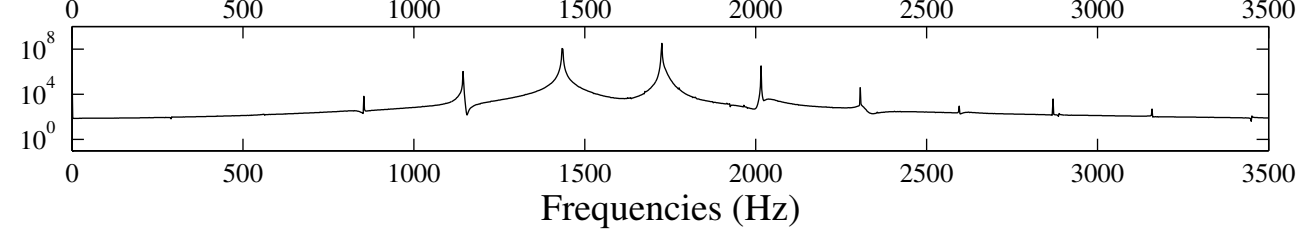

Figure 5.12: Simulated dynamics modulation as a function of $f_{i}$, power spectra. The driving frequency, $f_{i}$, was 1685 (a), 1690 (b), 1700 (c), 1705 (d), 1715 (e), and 1725 (f). Other parameters were $M$ toggling between 0.02 (when high mode detected) and 0.04 (when low mode detected), $\epsilon=0.1$, and $\beta=1$. The coupling parameters were $\gamma_{1}=0.025$ and $\gamma_{2}=0.22$. The mode frequencies were set at $\omega_{1}=2 \pi \times 1450 \mathrm{~Hz}$ and $\omega_{2}=2 \pi \times 1740 \mathrm{~Hz}$. 

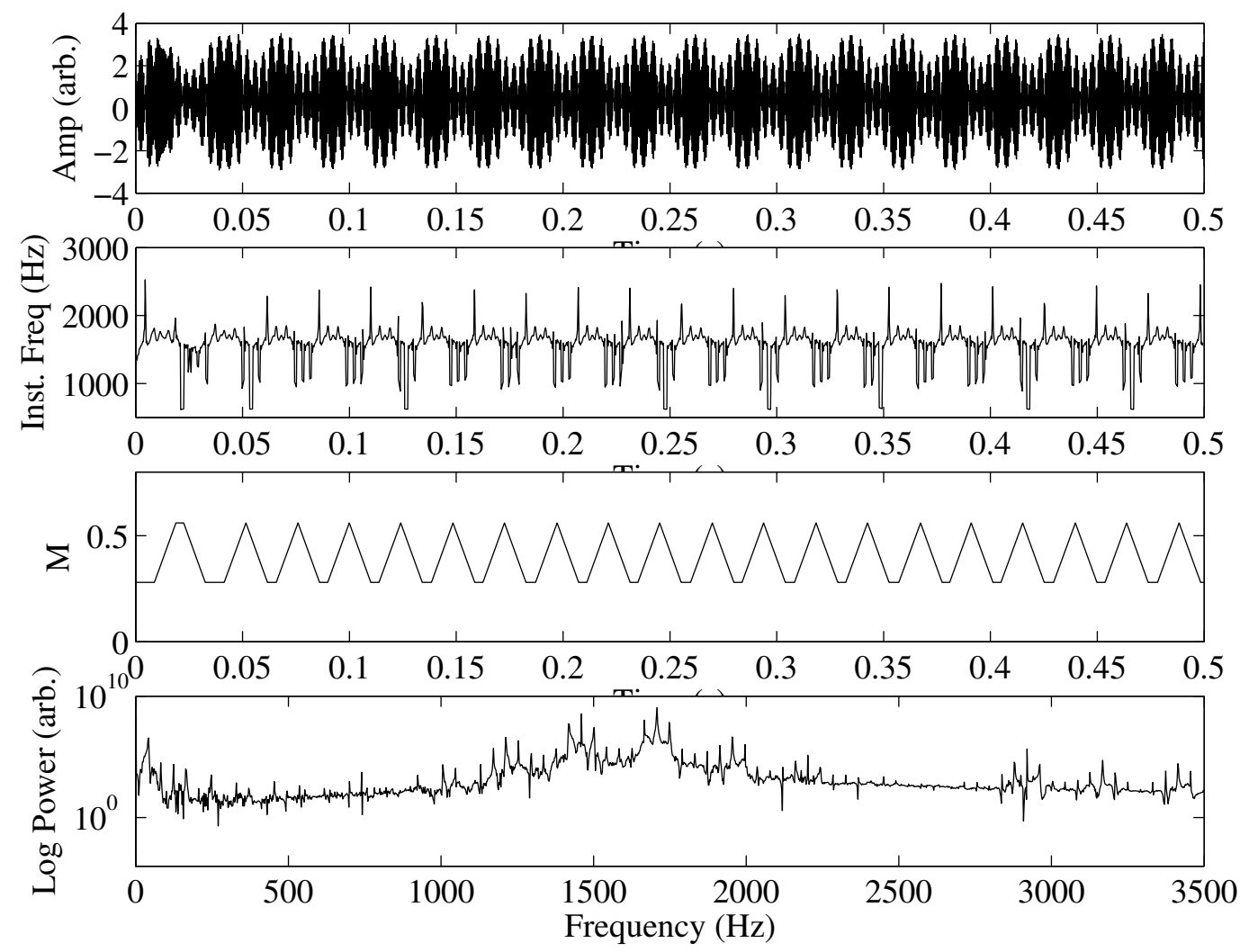

Figure 5.13: Symmetric Downward Modulation. This behavior was produced with the same $\epsilon(0.1)$, $\beta(1)$, and coupling parameters $\left(\gamma_{1}=0.025\right.$ and $\left.\gamma_{2}=0.22\right)$ as the upward case. $M$ was toggled between 0.28 (when the low mode was detected) and 0.56 (when the high mode was detected). The mode frequencies were set at $\omega_{1}=2 \pi \times 1703 \mathrm{~Hz}$ and $\omega_{2}=2 \pi \times 1455 \mathrm{~Hz}$. The driving frequency was set at $f_{i}=1460 \mathrm{~Hz}$. A similar symmetric case of downward modulation was not seen in the experiment. The panels, in order from top to bottom, show the time series, the instantaneous frequency, the value of $M$ used in the simulation in response to the detected mode, and the power spectrum. 


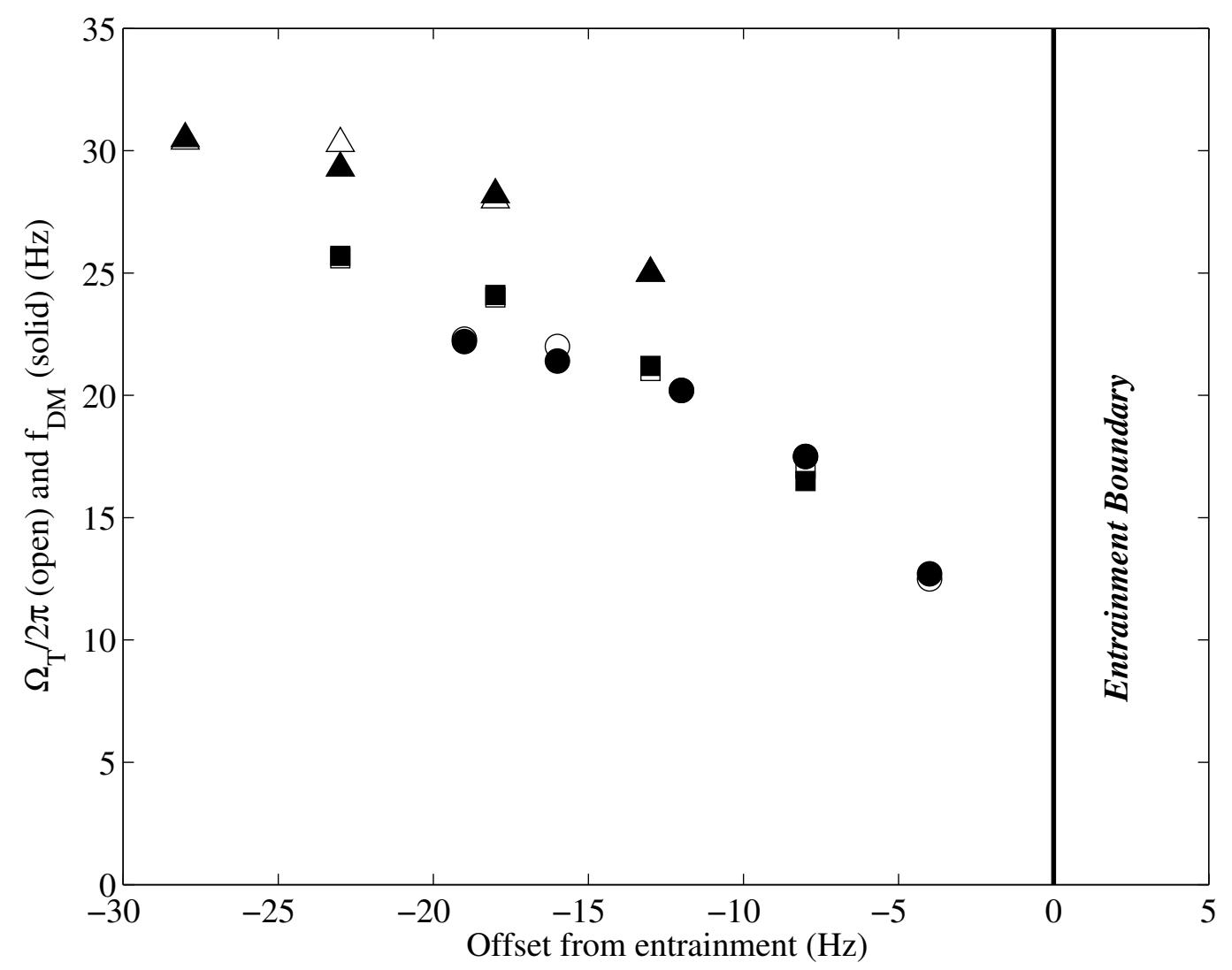

Figure 6.1: Dynamics modulation occurs at the temporal pulling beat frequency. This figure compares data from the temporal periodic pulling beat frequency (open), obtained from the low frequency peak on the power spectrum in the manner of Figure 4.14 with the periodic dynamics modulation frequency (filled), measured from the time series. This figure shows that the two frequencies are identical. Circles and squares represent data sets one and two respectively, modulating from $f 7$ to $f 8$. Triangles represent data set 3 , modulating from $f 8$ to $f 9$. 


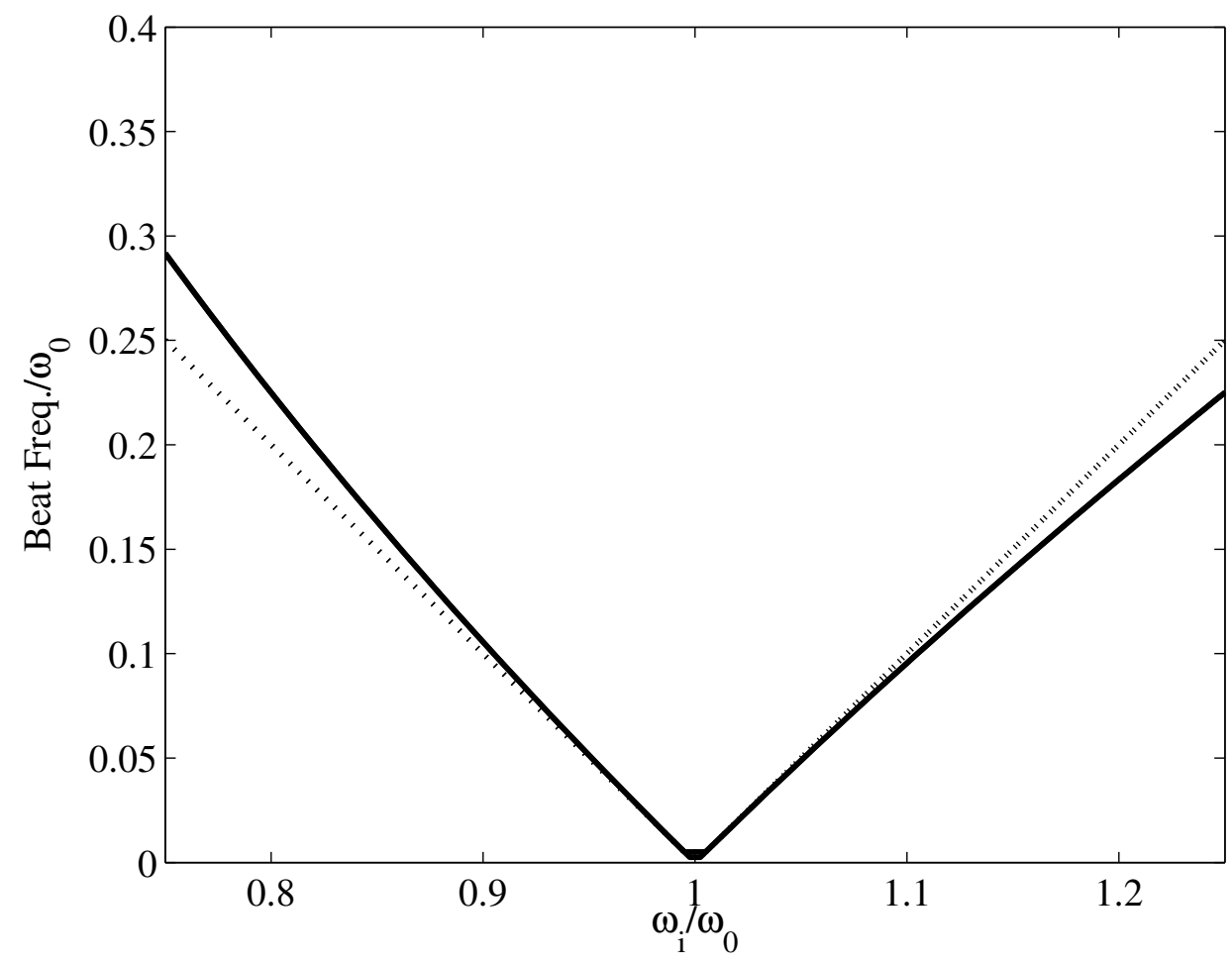

Figure 6.2: Asymmetry in reference beat frequency between two separated modes. The dotted lines represent the conventional beat frequency, $\Omega_{0}=\left|\omega_{i}-\omega_{0}\right|$. The reference beat frequency, $\Omega^{\prime}$ (calculated from Eq. (2.20)) is shown with the solid lines. For two modes separated significantly, this figure predicts that the beat frequency will be higher when the lower frequency pulls the higher frequency than when the opposite is true. 


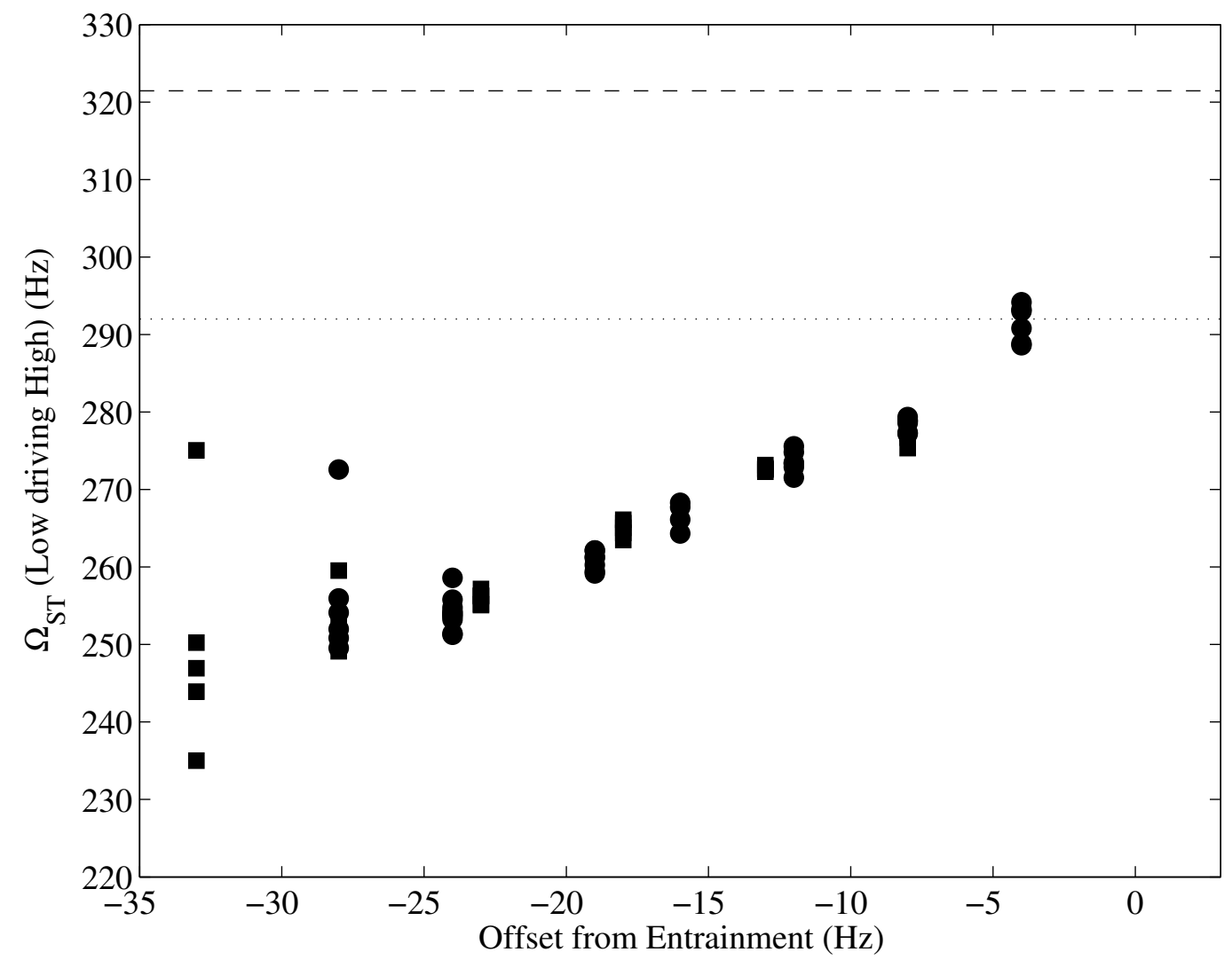

Figure 6.3: Upper beat frequency for low mode pulling high mode. This figure is the complementary figure to Figure 4.15 (top) for the high mode pulling the low mode. Measured beat frequencies are higher in this case. The dotted line is the conventional beat frequency $\Omega_{0}$ and dashed line is the reference beat frequency $\Omega^{\prime}$. In this case, since $\Omega^{\prime}$ is calculated based on the two mode frequencies only, it is not dependent on driving frequency. 

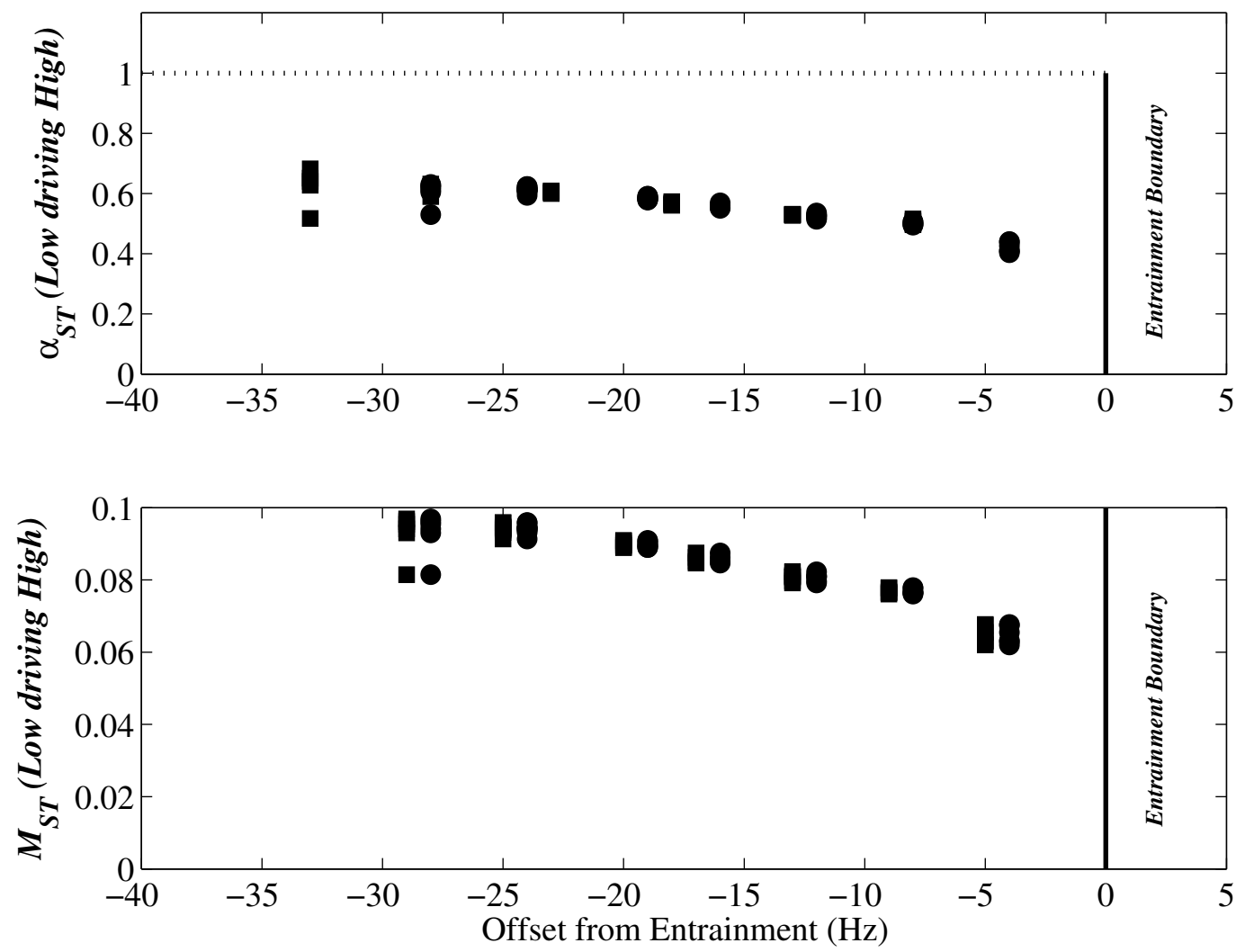

Figure 6.4: $\alpha$ and $M$ for the low mode pulling down on the high mode. This figure is the complementary figure to Figure 4.16 (left half) for the high mode pulling the low mode. Measured $\alpha$ and $M$ values are significantly higher in this case, reflecting the system's strong preference for the $f 7$ mode. The solid lines to the right indicate the entrainment boundary for the $f 8$ mode. The dotted line in the top panel indicates the entrainment boundary for the $f 7$ mode, which is never approached. 

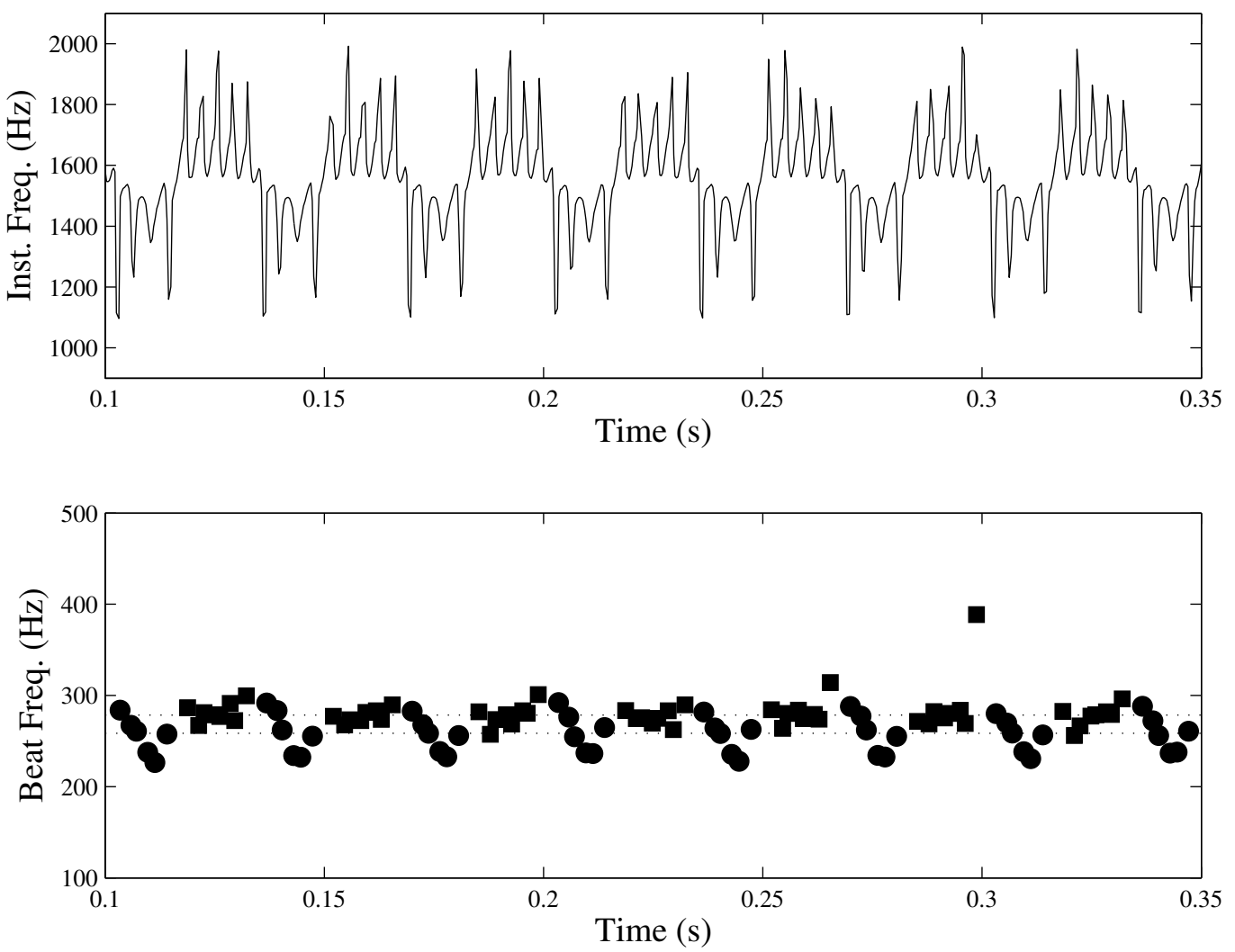

Figure 6.5: Beat separation from the model. This figure shows that the model successfully predicts a shift in beat frequencies between the two parts of the dynamics modulation cycle. The driving frequency in this case was set to $1700 \mathrm{~Hz}$. The dashed lines in the bottom panel occur at the median frequencies of the lower $(\bullet, 258.6 \mathrm{~Hz})$ and higher $(\boldsymbol{\square}, 278.4 \mathrm{~Hz})$ beat frequencies. Compare this figure to the experimental case shown in Figure 4.14. 

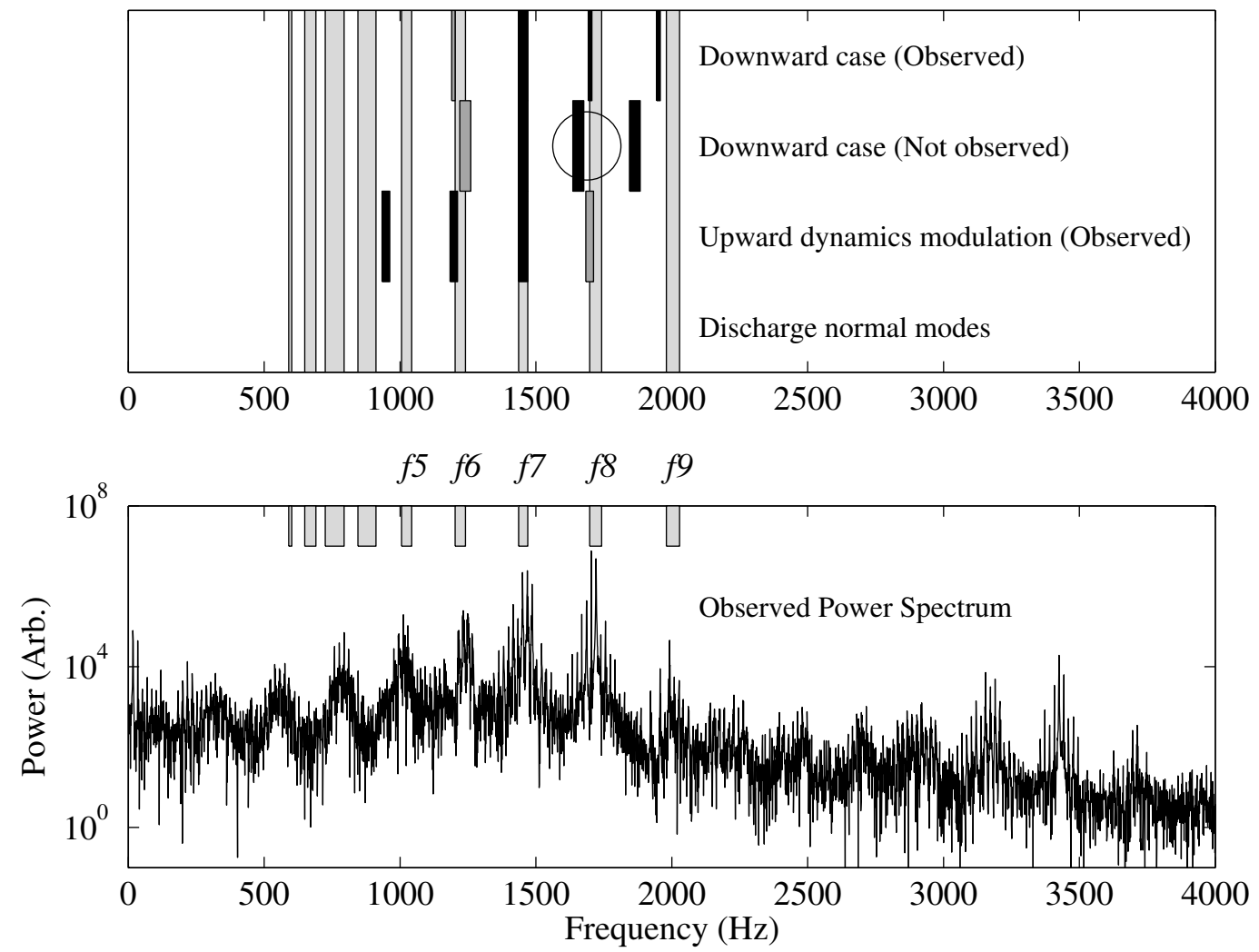

Figure 6.6: Multimode interaction may assist upward dynamics modulation but not the symmetric downward case. The bottom panel shows an observed downward dynamics modulation spectrum. For reference, light gray bars are placed at the observed mode frequencies for this discharge tube, and extended into the top panel. Medium gray rectangles are placed at driving frequencies used for dynamics modulation attempts as labeled. The black rectangles represent the first two expected sidebands opposite the driven mode. In the two successful cases, the first sideband overlaps an existing mode. For the symmetric downward modulation case, for which no dynamics modulation was observed, there is no overlap (circle). 